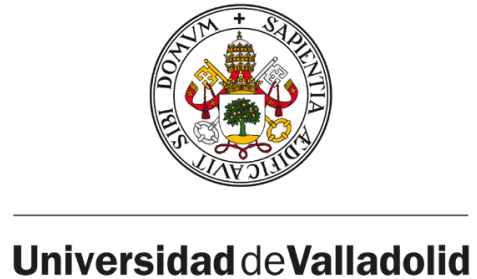

Universidad deValladolid

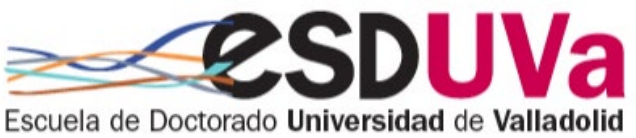

Escuela de Doctorado Universidad de Valladolid

\author{
PROGRAMA DE DOCTORADO EN \\ INVESTIGACIÓN EN CIENCIAS DE LA SALUD
}

TESIS DOCTORAL

\title{
CONECTIVIDAD DE LA RED CEREBRAL EN \\ EL TRASTORNO BIPOLAR Y LA ESQUIZOFRENIA
}
Presentada por Béquer Benjamín Cea Cañas para optar
al grado de

Doctor por la Universidad de Valladolid

Dirigida por

Dr. Vicente Molina Rodríguez

$y$

Dr. Álvaro Díez Revuelta

2021

Valladolid, España 

Título: Conectividad de la red cerebral en el trastorno bipolar y la esquizofrenia

Autor: Béquer Benjamín Cea Cañas

Dirigida por: Dr. Vicente Molina Rodríguez y Dr. Álvaro Díez Revuelta

Tutorizada por: Dr. Vicente Molina Rodríguez

Departamento: Pediatría e Inmunología, Obstetricia y Ginecología, Nutrición y Bromatología, Psiquiatría e Historia de la Ciencia

Esta tesis fue leída en la Facultad de Medicina de la Universidad de

Valladolid el día , estando compuesto el tribunal

calificador por :

Presidente:

Secretario:

Vocal:

Obteniendo la calificación de:

Valladolid, de de 2021 

A mi

Padre

y a mi madre 



\section{Agradecimientos}

Con emoción, quiero agradecer a todos los que de un modo u otro me han acompañado en la elaboración de esta tesis. En primer lugar, a Vicente Molina: sin él habría sido imposible. No encuentro las palabras para agradecerle su apoyo, por darme la oportunidad de trabajar bajo su supervisión, compartir conmigo sus ideas y proveerme de los medios para su realización. Su ejemplo de dedicación a la investigación ha sido una fuente de confianza y ánimo para iniciar y concluir este trabajo.

Mi gratitud se extiende a Álvaro Díez, quien también tuvo la disposición de dirigirme en esta tesis y me ayudó cada vez que lo necesité. Esta tesis también se debe a sus instrucciones y su guía.

Agradezco a mis amigos y compañeros de la Universidad de Valladolid, quienes con su esfuerzo y perseverancia en la consecución de sus metas fueron para mí ejemplos a imitar. A Alba Lubeiro, Javier Gómez, Alejandro Bachiller y Oscar Martín; gracias por compartir conmigo sus conocimientos, asesorarme y animarme en mi proyecto. A Sabela Fondevila, Henar de la Red, Inés Fernández, Rosa Beño, Álvaro Planchuelo y demás compañeros que formaron o forman parte del grupo SUCEDE, por la ayuda, colaboración y compañía, que han hecho agradable el tiempo compartido.

También quiero agradecer a los coautores de los artículos científicos que componen esta tesis, a Rodrigo de Luis, Eva Sotelo, Pilar del Valle, Marta Gómez, Adrián Alonso, Pablo Núñez, Eva Rodríguez, Nieves de Uribe, Adela Pérez, María Iglesias, Carmen Capella y Alberto Rodríguez. Estas publicaciones fueron el resultado del trabajo en equipo y sus contribuciones fueron decisivas. Ha sido un honor trabajar con ellos.

Gracias a Margarita Rodríguez y al equipo de la Unidad de Resonancia Magnética. Así también, a Marta Ayuso y al resto de mis compañeros de la sección de Neurofisiología del Hospital Clínico Universitario de Valladolid. 
De manera especial, quiero dar las gracias a todos cuantos participaron en los estudios, pacientes y controles, por su aportación desinteresada y generosa a la investigación sin la cual este trabajo no se habría hecho.

$\mathrm{Y}$ a tantas otras personas que con sus palabras y actos me dieron su apoyo para poder llegar hasta aquí. Confío en tener la oportunidad de hacerles saber lo importantes que fueron y son para mí.

¡Muchas gracias a todos por ayudarme a conseguirlo! 


\section{Resumen}

La esquizofrenia y el trastorno bipolar son enfermedades mentales que presentan alteraciones de la conectividad cerebral. En esta tesis se examinan las anormalidades de la red cerebral — tanto estructural como funcional- en estos dos trastornos a fin de determinar su especificidad para el diagnóstico y su posible relación con la duración de la enfermedad y el tratamiento farmacológico.

Las redes estructurales se basan en datos obtenidos por imagen por tensor de difusión y las funcionales en electroencefalogramas (EEG) registrados durante una tarea cognitiva. La conectividad global de estas redes se estudia con parámetros de la teoría de grafos: la fuerza de conectividad (CS) e índice de mundo pequeño (SW). Asimismo, dado que la conectividad funcional entre grupos neuronales se fundamenta en la interacción de sus oscilaciones, se estudia la actividad oscilatoria en la banda $\gamma$ del EEG $(35-45 \mathrm{~Hz})$ a través de la potencia del ruido cortical (NP).

Los resultados muestran una reducción de los parámetros de conectividad estructural (CS y SW) en la esquizofrenia crónica y en el trastorno bipolar. En cuanto al parámetro de conectividad funcional (CS) calculado durante una tarea cognitiva, se observa un aumento en la ventana preestímulo en la esquizofrenia y una reducción de su modulación en la ventana respuesta en ambas enfermedades. Con respecto a la NP en la banda $\gamma$, se observa un aumento de su valor en la esquizofrenia, y en menor medida, en el trastorno bipolar.

Estas alteraciones se presentan con más frecuencia e intensidad en la esquizofrenia, lo que sugiere una mayor especificidad para este síndrome en comparación con el trastorno bipolar. Ninguna de estas alteraciones muestra relación con el tratamiento farmacológico, y solo se encuentra una relación con significación estadística entre la duración de la enfermedad y los parámetros de conectividad estructural en los pacientes con esquizofrenia. 



\section{Abstract}

Schizophrenia and bipolar disorder are mental disorders with brain connectivity alterations. In this thesis we examine brain network abnormalities - structural and functional - in these two disorders in order to determine their specificity for diagnosis and their possible relationship to disease duration and pharmacological treatment.

Structural networks are based on diffusion tensor imaging data and functional networks on electroencephalograms (EEG) recorded during a cognitive task. The global connectivity of these networks is studied with graph theory parameters: connectivity strength (CS) and small-world index (SW). Also, since the functional connectivity between neuronal groups is based on the interaction of their oscillations, the oscillatory activity in the $\gamma$-band of the EEG $(35-45 \mathrm{~Hz})$ is studied through the cortical noise power (NP).

The results show a reduction of structural connectivity parameters (CS and SW) in chronic schizophrenia and bipolar disorder. As for the functional connectivity parameter (CS) calculated during a cognitive task, an increase is observed in the pre-stimulus window in schizophrenia and a reduction of its modulation in the response window in both disorders. Regarding the NP in the $\gamma$-band, an increase in its value is observed in schizophrenia, and to a lesser extent, in bipolar disorder.

These alterations occur with greater frequency and intensity in schizophrenia, suggesting a greater specificity for this syndrome compared to bipolar disorder. None of these alterations shows a relationship with pharmacological treatment, and only a statistically significant relationship is found between the duration of the disease and structural connectivity parameters in patients with schizophrenia. 



\section{Presentación}

En esta tesis doctoral se examinan las alteraciones de la conectividad de la red cerebral estructural y funcional en la esquizofrenia y el trastorno bipolar. Su aportación original consiste en hacer comparaciones directas de los parámetros de conectividad de las redes globales entre estos dos trastornos para determinar la especificidad de las alteraciones para el diagnóstico, así como analizar su posible relación con la duración de la enfermedad y el tratamiento farmacológico. Los resultados obtenidos son relevantes porque amplían la descripción y caracterización de las anormalidades de la red cerebral presentes en los trastornos psicóticos.

Se sigue la modalidad por compendio de publicaciones y, por lo tanto, consta de tres estudios publicados en sendos artículos científicos. El primero analiza la conectividad estructural o anatómica en redes elaboradas con datos obtenidos a partir de imágenes por resonancia magnética ponderada en difusión. El segundo examina la conectividad funcional de redes basadas en encefalogramas registrados durante una tarea cognitiva experimental. El tercero se dedica al análisis de un parámetro que estudia la actividad oscilatoria en la banda $\gamma$ del EEG durante una tarea cognitiva, dado que las conexiones corticales funcionales 
a nivel local se fundamentan en oscilaciones rítmicas en esta banda. Los artículos que contienen estos estudios son, respectivamente:

1. «Structural connectivity in schizophrenia and bipolar disorder: Effects of chronicity and antipsychotic treatment»

Autores: Benjamín Cea-Cañas, Rodrigo de Luis, Alba Lubeiro, Javier Gomez-Pilar, Eva Sotelo, Pilar del Valle, Marta Gómez-García, Adrián Alonso-Sánchez y Vicente Molina.

Publicado en: Progress in Neuro-Psychopharmacology and Biological Psychiatry. Volumen 92, 8 de junio de 2019, páginas 369-377.

Base de indexación: Journal Citation Reports, Clarivate 2021

Factor de impacto: 4.361. Cuartil JIF Q1 Psychiatry (31/155), Pharmacology \& Pharmacy Q1 (45/271).

DOI: 10.1016/j.pnpbp.2019.02.006

2. "Connectivity strength of the EEG functional network in schizophrenia and bipolar disorder»

Autores: Benjamín Cea-Cañas, Javier Gomez-Pilar, Pablo Núñez, Eva Rodríguez-Vázquez, Nieves de Uribe, Álvaro Díez, Adela PérezEscudero y Vicente Molina.

Publicado en: Progress in Neuro-Psychopharmacology and Biological Psychiatry. Volumen 98, 2 de marzo de 2020, 109801.

Factor de impacto: 5.067. Cuartil JIF Q1 Psychiatry (35/156), Pharmacology \& Pharmacy Q1 (62/275).

DOI: 10.1016/j.pnpbp.2019.109801

3. "Altered gamma band noise power in schizophrenia and bipolar patients during a cognitive task»

Autores: Benjamín Cea-Cañas, Álvaro Díez, Alba Lubeiro, María Iglesias, Carmen Capella, Alberto Rodríguez-Lorenzana y Vicente Molina.

Publicado en: European Journal of Psychiatry. Volumen 35, número 3, páginas 129-139 (julio-septiembre 2021).

Factor de impacto: 0.673. Cuartil JIF Q4 Psychiatry (134/143)

DOI: $10.1016 / j$.ejpsy.2020.11.003 
Se desarrolla una línea de investigación del grupo Sustratos Cerebrales de la Psicosis (SUCEDE), un equipo multidisciplinar dedicado al estudio de los trastornos mentales graves que dirige el Prof. V. Molina. El tema y plan general de esta tesis es fruto de su trabajo intelectual. La realización de las investigaciones publicadas en los tres artículos que la conforman ha sido posible gracias a las becas y ayudas a la investigación del Instituto de Salud Carlos III (PI15/00299 y PI18/00178), de la Gerencia Regional de Castilla y León (GRS 1485/A/17 y GRS 1721/A/18) y de la Consejería de Educación de la Junta de Castilla y León (VA059U13 y VA057P17).

IV

Esta memoria de tesis se divide en siete capítulos y una sección de anexos. En el capítulo 1 se expone la relevancia del tema y la consistencia temática de los tres artículos que la conforman. Además, se revisan los conceptos teóricos y los antecedentes más importantes. En el capítulo 2 se detallan las hipótesis y los objetivos. En el capítulo 3 se ofrece una síntesis de la metodología utilizada en los tres estudios. En el capítulo 4 se describen los principales resultados y el capítulo 5 se dedica a su discusión; en ambos casos se hace una adaptación de la traducción al español de las respectivas secciones de cada una de las publicaciones. En el capítulo 6 se presentan las conclusiones generales de la investigación y el capítulo 7 contiene las referencias bibliográficas. Por último, la sección de anexos comprende el texto íntegro de los tres artículos científicos en la versión aceptada por las revistas en que fueron publicados.

A fin de facilitar la fluidez de la lectura del texto y para evitar desdoblamientos o reiteraciones, se emplea el género masculino con valor genérico. Por consiguiente, se usa como forma no marcada del castellano para la denominación de las personas, refiriéndose indistintamente a hombres y mujeres. 



\section{Índice general}

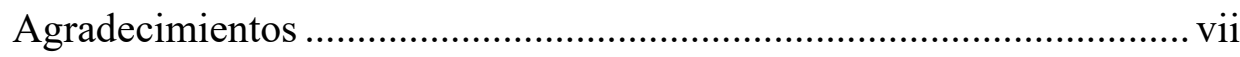

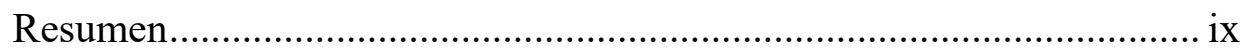

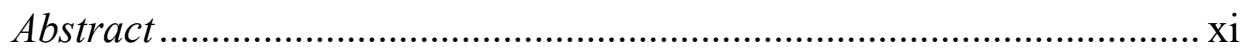

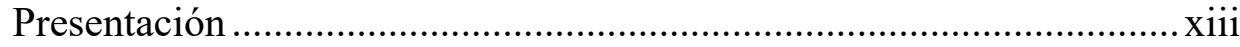

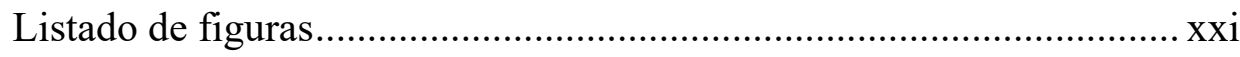

Listado de tablas .................................................................................

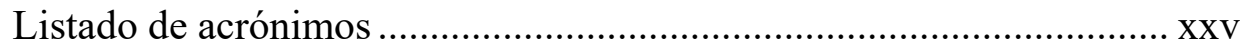

Capítulo 1 Introducción ...................................................................... 1

1.1. Esquizofrenia y trastorno bipolar ........................................... 3

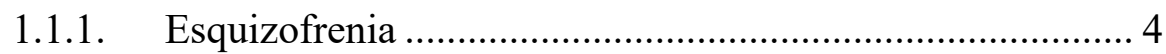

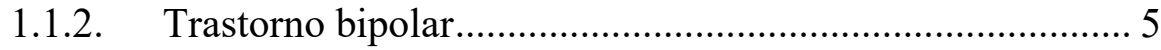

1.2. Redes cerebrales: de tejido nervioso a objetos matemáticos...... 6

1.2.1. Conectividad cerebral estructural y funcional .................... 8

1.2.2. Neurociencia de redes: la teoría de redes complejas aplicada al estudio del cerebro

1.2.3. Teoría de grafos: las matemáticas en las redes cerebrales 10

1.3. Oscilaciones cerebrales: conexión neuronal a través de la

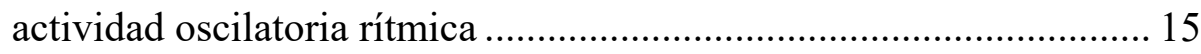

1.3.1. Osciladores cerebrales y bandas de frecuencia................. 17

1.3.2. Sincronización neuronal: conexión por medio de las oscilaciones cerebrales 18 
Índice general

1.3.3. Tipos de oscilaciones: actividad espontánea y potenciales relacionados con eventos ............................................................. 20

1.3.4. Potencia de ruido cortical (NP Noise power).................... 21

1.4. Instrumentos para la adquisición de los datos de conectividad 23

1.4.1. Resonancia magnética ponderada en difusión ................... 23

1.4.1.1. Elipsoide y tensor de difusión ................................... 25

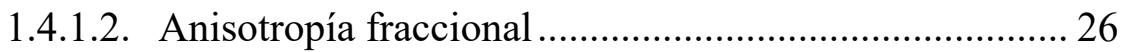

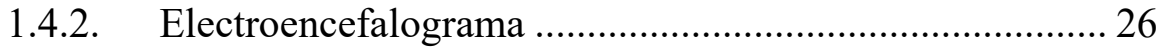

1.5. Estado de la cuestión ........................................................... 28

1.5.1. Conectividad estructural: análisis de la CS y del SW ....... 28

1.5.2. Conectividad funcional: análisis de la CS y su modulación durante una tarea cognitiva............................................................ 29

1.5.3. Actividad oscilatoria $\gamma$ : análisis de la NP ....................... 30

Capítulo 2 Hipótesis y objetivos ........................................................... 31

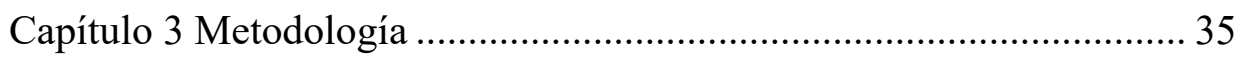

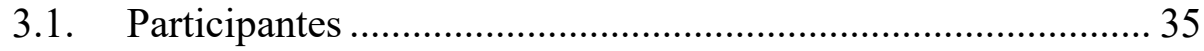

3.2. Valoración de la cognición, de los síntomas y del tratamiento farmacológico

3.3. Adquisición y procesamiento de las imágenes por resonancia magnética ponderada en difusión ..................................................... 38

3.4. Adquisición de los electroencefalogramas durante un paradigma

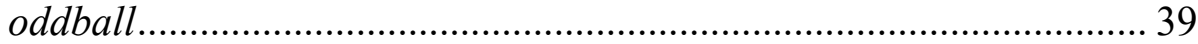

3.4.1. Procesamiento del EEG y elaboración de las matrices de conectividad funcional

3.4.2. Procesamiento del EEG para el cálculo del ruido cortical 41

3.5. Cálculo de los parámetros para la valoración de la conectividad de la red y la actividad oscilatoria ..................................................... 42

3.5.1. Cálculo de los parámetros de la teoría de grafos .............. 42

3.5.2. Cálculo de potencia de ruido en la banda $\gamma(\mathrm{NP})$............... 44

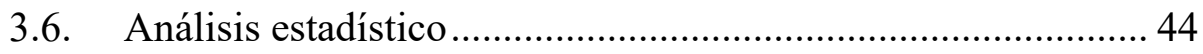

3.6.1. Artículo 1: Structural connectivity in schizophrenia and bipolar disorder: Effects of chronicity and antipsychotic treatment 45 


\subsubsection{Artículo 2: Connectivity strength of the EEG functional} network in schizophrenia and bipolar disorder

3.6.3. Artículo 3: Altered gamma band noise power in schizophrenia and bipolar patients during a cognitive task

Capítulo 4 Resultados

4.1. Conectividad estructural de la red cerebral

4.1.1. Resultados demográficos, clínicos y cognitivos

4.1.2. Especificidad para el diagnóstico de las alteraciones de los parámetros de conectividad estructural

4.1.3. Relación de las alteraciones de los parámetros de conectividad estructural con la duración de la enfermedad..

4.1.4. Relación de las alteraciones de los parámetros de conectividad estructural con el tratamiento farmacológico

4.1.5. Relación de las alteraciones de los parámetros de conectividad estructural con la cognición y los síntomas.

4.2. Conectividad funcional de la red cerebral............................... 56

4.2.1. Resultados demográficos, clínicos y cognitivos ................ 56

4.2.2. Especificidad para el diagnóstico de las alteraciones en los parámetros de conectividad funcional .............................................. 58

4.2.3. Relación de las alteraciones de los parámetros de conectividad funcional con la duración de la enfermedad

4.2.4. Relación de las alteraciones de los parámetros de conectividad funcional con el tratamiento farmacológico.

4.2.5. Relación de las alteraciones de los parámetros de conectividad funcional con la cognición y los síntomas

4.3. Potencia de ruido cortical en la banda $\gamma(\mathrm{NP}-\gamma)$......................... 64

4.3.1. Resultados demográficos, clínicos y cognitivos ................ 64

4.3.2. Especificidad para el diagnóstico de las alteraciones de la

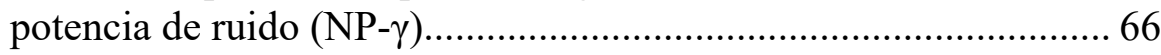

4.3.3. Relación de las alteraciones de la NP- $\gamma$ con la duración de la enfermedad 68

4.3.4. Relación de las alteraciones de la NP- $\gamma$ con el tratamiento farmacológico

4.3.5. Relación de las alteraciones de la NP- $\gamma$ con la cognición y los síntomas 69 
Índice general

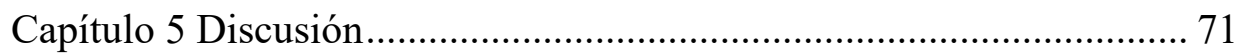

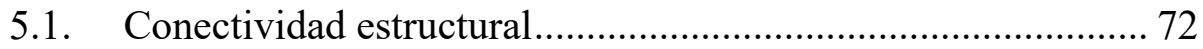

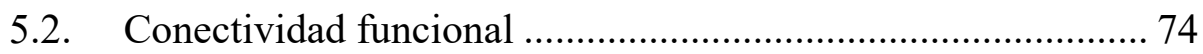

5.3. Potencia de ruido cortical en la banda $\gamma(\gamma-\mathrm{NP}) \ldots \ldots \ldots \ldots \ldots \ldots \ldots . . . . . . . . . . . .77$

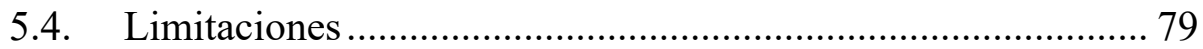

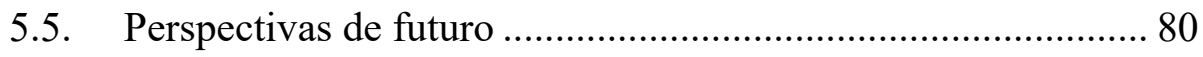

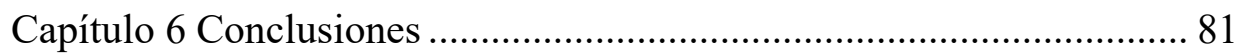

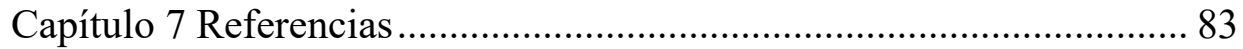

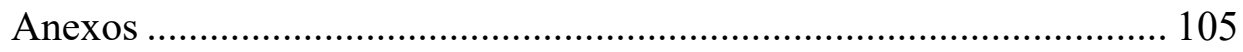

Anexo 1 Structural connectivity in schizophrenia and bipolar disorder: Effects of chronicity and antipsychotic treatment .... 107 Anexo 2 Connectivity strength of the EEG functional network in schizophrenia and bipolar disorder.

Anexo 3 Altered gamma band noise power in schizophrenia and bipolar patients during a cognitive task. 


\section{Listado de figuras}

Fig. 1 Redes cerebrales en las escalas temporal y espacial............. 7

Fig. 2| Modelos de redes cerebrales.................................... 10

Fig. 3 | Elaboración de los grafos cerebrales............................12

Fig. 4 | Parámetros de la teoría de grafos..............................13

Fig. 5 Parámetros que definen una señal oscilatoria e interacción entre las oscilaciones cerebrales..........................................19

Fig. 6| Tractografía por tensor de difusión............................24

Fig. 7| Electroencefalograma (EEG) .................................27

Fig. 8| Fuerza de conectividad e índice de mundo pequeño de la red

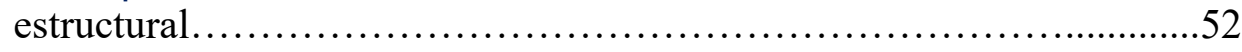

Fig. 9 | Relación de los valores de fuerza de conectividad e índice de mundo pequeño con la duración de la enfermedad y el tratamiento

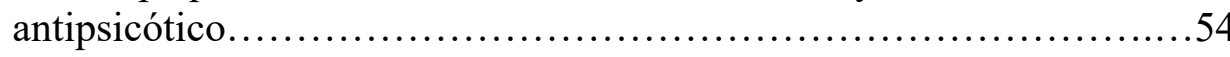

Fig. 10 Relación de los valores del índice de mundo pequeño con las puntuaciones cognitivas en la esquizofrenia...........................56

Fig. $11 \mid$ Fuerza de conectividad (CS) de la red funcional................59

Fig. 12 Modulación de la fuerza de conectividad durante la tarea cognitiva..................................................... 60

Fig. 13 | Relación de la fuerza de conectividad y su modulación con el

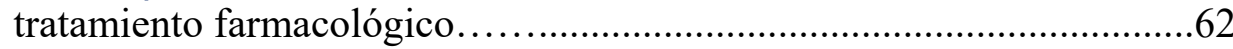

Fig. 14 | Relación de la fuerza de conectividad con las puntuaciones cognitivas y los síntomas en la esquizofrenia. 
Listado de figuras

Fig. 15 Potencia de ruido en la banda $\gamma$ en la esquizofrenia y el trastorno bipolar. .66

Fig. 16 Relación de la potencia de ruido en la banda $\gamma$ y el tratamiento

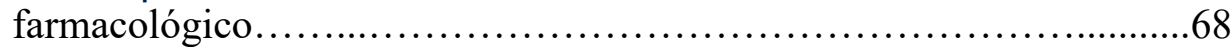

Fig. 17| Relación de la potencia de ruido en la banda $\gamma$ y los síntomas en los pacientes con esquizofrenia......................................70 


\section{Listado de tablas}

Tabla 1| Aspectos metodológicos de los artículos de investigación.......36

Tabla 2 Resultados demográficos, cognitivos, clínicos y del tratamiento en el estudio de conectividad estructural............................51

Tabla 3 | Valores de fuerza de conectividad (CS) e índice de mundo

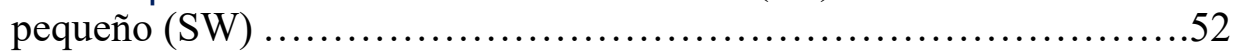

Tabla 4 | Resultados demográficos, cognitivos, clínicos y del tratamiento en el estudio de conectividad funcional..............................57

Tabla 5 | Valores de fuerza de conectividad (CS) en la ventana preestímulo y su modulación durante la tarea cognitiva en las bandas

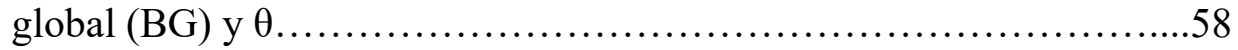

Tabla 6 Resultados demográficos, cognitivos, clínicos y del tratamiento en el estudio de la potencia de ruido cortical en la banda $\gamma$...................65

Tabla $7 \mid$ Valores de la potencia de ruido cortical en la banda $\gamma(\mathrm{NP}-\gamma) .67$ 



\section{Listado de acrónimos}

BACS Escala de evaluación breve de la cognición en la esquizofrenia (Brief Assessment in Cognition in Schizophrenia Scale)

BG Banda global

BZD Benzodiacepinas

CI Cociente intelectual

C Coeficiente de agrupamiento (Clustering coefficient)

$\mathrm{CPZ} \quad$ Clorpromazina

CS Fuerza de conectividad (Connectivity strength)

CWT Transformada wavelet continua (Continuous wavelet transform)

CTRL Controles sanos

HDRS Escala de evaluación para la depresión de Hamilton (Hamilton Depression Rating Scale).

DMN Red neuronal por defecto o de modo predeterminado (Default mode network)

DWI Imagen por resonancia magnética ponderada en difusión (Diffusion weighted imaging)

DTI Imagen por tensor de difusión (Diffusion tensor imaging)

EC Esquizofrenia crónica

EEG Electroencefalograma

EPE Esquizofrenia en el primer episodio

EPI Echo-Planar Imaging

ESQ Esquizofrenia 
FA

ICA

$\mathrm{L}$

MSCEIT Prueba de inteligencia emocional de Mayer-SaloveyCaruso (MSCEIT Mayer-Salovey-Caruso Emotional Intelligence Test)

NP Potencia de ruido cortical (Noise power)

PANSS Escala para el síndrome positivo y negativo de la esquizofrenia (Positive and Negative Syndrome Scale).

PFC Corteza prefrontal (Prefrontal cortex)

PLV Valor de acoplamiento de fase (Phase-locking value)

RM Resonancia magnética

SD Desviación estándar (Standard deviation)

SW Índice de mundo pequeño (Small worldness)

TB Trastorno bipolar

TFE Turbo Field Echo

WAIS-III Escala de inteligencia del adulto de Wechsler - tercera edición (WAIS-III Wechsler Adult Intelligence ScaleThird Edition)

WCST Test de clasificación de tarjetas de Wisconsin (Wisconsin Card Sorting Test)

YMRS Escala de manía de Young (Young Mania Rating Scale) 


\section{CAPÍTUL0 1}

\section{Introducción}

a aplicación de la teoría de redes complejas al estudio del cerebro

humano ha supuesto un gran avance para comprender los mecanismos de trasmisión y procesamiento de la información que sustentan los procesos cognitivos (1). De igual manera, ha proporcionado una nueva perspectiva para examinar las alteraciones fisiopatológicas que tienen lugar en diversas enfermedades neurológicas y psiquiátricas, entre las que se encuentran los trastornos psicóticos (2,3). Muchas investigaciones señalan la existencia de alteraciones en la conectividad de la red cerebral en estos trastornos (4), en particular en la esquizofrenia $(5,6)$, aunque también en el trastorno bipolar (7-9), algunas de las cuales se han relacionado con déficit cognitivos y síntomas clínicos (10).

En esta tesis se busca estudiar la conectividad de la red cerebral en el trastorno bipolar y la esquizofrenia con una metodología uniforme con el fin de determinar, por un lado, la especificidad de las alteraciones para estos diagnósticos y por otro, estudiar su posible relación con la duración de la enfermedad y con el tratamiento farmacológico. La investigación de la conectividad estructural y funcional se hace usando la red cerebral global, porque se considera que este es un modelo adecuado para el estudio de estos trastornos, que se manifiestan con síntomas que afectan las funciones mentales, las cuales se basan probablemente en la interacción de grandes grupos de neuronas localizados en amplias regiones cerebrales. Por otra parte, se reconoce que las conexiones de circuitos neuronales corticales a nivel local establecidas por medio de la actividad oscilatoria 
en la banda $\gamma$ también son fundamentales en los procesos cognitivos (1113), por lo que su valoración resulta igualmente de interés (14-17) y complementa la investigación de la conectividad de la red global en estas enfermedades.

El análisis de la conectividad de la red estructural se hace con dos parámetros derivados de la teoría de grafos, que proporcionan una descripción cuantitativa y cualitativa de la arquitectura conectiva $(2,18-$ 20). El primero es la fuerza de conectividad (CS, connectivity strength), que se calcula promediando los pesos de todas las aristas que hay en una red y valora la intensidad con la que los nodos están conectados dentro de la red, por lo que puede ser considerado como un marcador de la conectividad global. El segundo es el índice del mundo pequeño (SW, small-worldness), que estima la segregación e integración de la red cerebral, es decir, la relación entre la conectividad local de conjuntos neuronales especializados -medido por el coeficiente de agrupamiento, $\mathrm{C}-\mathrm{y}$ la comunicación entre regiones cerebrales distantes — medida por la longitud de camino característica, L- (21), de manera que equivale a un indicador de la eficiencia de la red.

El estudio de la conectividad de la red funcional se hace a través del parámetro CS durante una tarea cognitiva $\mathrm{P} 300$ con un paradigma oddball. En esta investigación la CS se estima en una condición preestímulo y durante la respuesta de la tarea. Esto permite calcular su modulación, definida como el cambio en este parámetro entre las condiciones preestímulo y respuesta, que posiblemente está relacionada con las demandas de la tarea cognitiva. En este caso la CS puede considerarse como una medida cuantitativa de la sincronía transitoria de las asambleas neuronales - grandes conjuntos de neuronas que participan de manera colectiva en los distintos procesos cerebrales distribuidos en amplias regiones de la corteza cerebral $(5,22-26)$ - y su modulación reflejaría probablemente la reorganización o remodelación de las conexiones funcionales requerida para la ejecución de la tarea.

El estudio de la actividad oscilatoria $\gamma$ también se realiza durante una tarea cognitiva P300, y el parámetro utilizado para su análisis es la potencia de ruido cortical (NP, noise power). Siguiendo una aproximación lineal, la actividad eléctrica total registrada por el EEG durante una tarea cognitiva estaría compuesta por la actividad vinculada con la tarea 
(enganchada en tiempo y fase con el estímulo) y por una actividad no vinculada con el estímulo pero que podría estar relacionada con otras actividades diferentes (es decir, el ruido cortical). El parámetro aquí usado para estudiar la actividad oscilatoria $\gamma$ es la NP, que precisamente estima la potencia de la actividad cortical que no está enganchada en tiempo y fase con el estímulo de la tarea experimental. Posiblemente la NP está relacionada con alteraciones de la señalización dopaminérgica y del equilibrio de los mecanismos excitadores e inhibidores (27-29), de los que hay evidencia en los trastornos psicóticos graves (30,31). De esta manera, la NP en la banda $\gamma$ puede considerarse como un indicador de la sincronía de la actividad de las asambleas neuronales y de la magnitud de otra actividad cortical no relacionada con la ejecución de la tarea $(32,33)$

\subsection{Esquizofrenia y trastorno bipolar}

El término $p s i c o s i s$, - del griego $p s y k h(\bar{e}) \psi \bar{v} \chi \dot{\eta}$, mente y - $\bar{o}$-sis, proceso patológico (34) - se refiere a un estado caracterizado por una alteración de la capacidad para distinguir entre la experiencia mental interna y la realidad externa del ambiente, que se acompaña de alteraciones del pensamiento, las emociones y la conducta, con una evolución variable a lo largo del tiempo $(35,36)$. En esta tesis, la conectividad cerebral se estudia en los dos trastornos psicóticos más representativos: la esquizofrenia y el trastorno bipolar tipo I. En esta sección se revisan sus principales características.

Como ocurre con otras enfermedades mentales, las causas y mecanismos que producen los trastornos psicóticos no son plenamente conocidos. Los resultados de numerosas investigaciones han encontrado diversas alteraciones biológicas, como las de tipo genético, neuropatológico, bioquímico e inmunológico (36-38). En las últimas décadas la aplicación de la teoría de redes complejas para estudiar los patrones de conectividad en estos trastornos, tanto de la red cerebral estructural como funcional, ha contribuido a ampliar la comprensión de la disfunción cerebral que ocurre en ellos $(5,39)$. 


\subsubsection{Esquizofrenia}

Más que una sola enfermedad, la esquizofrenia es probablemente un espectro de trastornos de naturaleza heterogénea (36). Esta idea ya estaba presente en 1911, cuando E. Bleuler - a quien se le atribuye la introducción del término esquizofrenia - distinguía entre los grupos nucleares y marginales, que diferían en la gravedad de los síntomas y en el pronóstico (40).

Sus síntomas afectan el pensamiento, las emociones y el comportamiento, e incluyen la pérdida de la verificación de la realidad, desorganización parcial de las funciones de la personalidad, tendencia al aislamiento social, delirios y alucinaciones. Puede tener un inicio repentino o gradual, y persiste a lo largo de la vida, con variación en la intensidad de los síntomas crónicos $(36,41,42)$.

Epidemiología y etiología. Es la forma más frecuente de trastorno mental grave, que afecta a alrededor de 1 persona de cada 100 (41), con inicio usualmente en la adolescencia o al comienzo de la edad adulta. En su etiología se han descrito diferentes factores relacionados con el inicio o con la vulnerabilidad a padecerla, que incluyen los de tipo biológico, como factores genéticos, neuroquímicos, infecciosos y anatomopatológicos tanto de las neuronas como de la neuroglía- (43), así como los de tipo psicológico y social (36).

Síntomas y diagnóstico. La sintomatología se agrupa en síntomas positivos y negativos, siendo común la coexistencia de ambos. Los síntomas positivos incluyen alucinaciones — con más frecuencia las de tipo auditivo, aunque cualquier modalidad sensorial puede estar involucrada - y los delirios, que son ideas irreales debidas a la distorsión subjetiva de las observaciones del mundo circundante (44). Los síntomas negativos abarcan el afecto plano o embotado, la alogia, la anhedonia, el aislamiento social y la falta de interés y motivación. Según el Manual diagnóstico y estadístico de los trastornos mentales en su $5^{\text {a }}$ edición (DSM-5), para su diagnóstico deben estar presentes al menos dos síntomas (como alucinaciones, delirios, habla desorganizada, comportamiento desorganizado o síntomas negativos) por un período mínimo de un mes y para su confirmación deben permanecer durante al menos seis meses (45). 


\subsubsection{Trastorno bipolar}

$\mathrm{Al}$ igual que la esquizofrenia, se considera que bajo la denominación de trastorno bipolar más que una única enfermedad se agrupa un espectro de trastornos con algunas características comunes, colindante con unos límites confusos con la esquizofrenia por un lado y con la depresión unipolar por otro (46).

Su clasificación se fundamenta en el curso clínico. El trastorno bipolar tipo I, llamado en el pasado psicosis maniaco-depresiva, es un trastorno recurrente definido por cambios del estado de ánimo y de la energía, con la presencia de episodios de manía, que con frecuencia incluye síntomas psicóticos graves (47).

Epidemiología y etiología. Los trastornos bipolares afectan a nivel mundial a cerca de 1 persona por cada 100. Sus inicios suelen ocurrir al final de la adolescencia o durante los primeros años de la edad adulta y son una causa importante de discapacidad en personas jóvenes $(48,49)$. Existe evidencia de una base genética (50) y, al igual que en la esquizofrenia, se han descrito diferentes alteraciones neuromorfológicas cerebrales y alteraciones neurobiológicas en el sistema de neurotransmisores y neuroendocrinos, entre otras anormalidades.

Síntomas y diagnóstico. Se caracteriza por variaciones extremas en el estado de ánimo, la motivación y la excitación, presentándose con episodios de exaltación del estado de ánimo y de la actividad motora (manía o hipomanía) y episodios de estado de ánimo depresivo, con pérdida del interés o de la capacidad por sentir placer en las actividades de la vida (depresión). Su diagnóstico a menudo se retrasa debido a que en ocasiones su presentación inicial es semejante a la depresión unipolar, por lo que la detección de los episodios de hipomanía/manía resulta crucial para su diagnóstico (49). Según el DSM-5, para el diagnóstico del trastorno bipolar tipo I se debe presentar al menos un episodio maníaco, mientras que para el tipo II se debe presentar al menos un episodio de hipomanía y un episodio depresivo mayor (45). 


\subsection{Redes cerebrales: de tejido nervioso a objetos matemáticos}

El cerebro humano recibe, procesa y trasmite información mediante el funcionamiento coordinado de un gran número de neuronas - cuyo número se estima entre 80 y 100 mil millones (51), interconectadas por unos 150 billones de sinapsis $(52,53)$ - . El estudio de su organización ha avanzado mucho desde las investigaciones que a finales del siglo XIX derivaron en la teoría reticular de J. Gerlash y C. Golgi y en la doctrina neuronal de S. Cajal, hasta la postulación del concepto de conectoma a inicios del siglo XXI. Sin embargo, la comprensión de cómo los elementos celulares logran la actividad colectiva que produce las funciones cerebrales sigue siendo todavía una cuestión sin una respuesta definitiva (54). El estudio de la red cerebral aplicando las herramientas de la teoría de grafos es un nuevo enfoque con el que se aborda este problema, que ofrece al mismo tiempo una nueva perspectiva para estudiar la fisiopatología de las enfermedades cerebrales.

Conocer la fisiología del cerebro humano y comprender cómo este órgano -que equivale a un 2 por 100 del peso promedio de una persona adultahace de cada uno lo que es, representa sin duda uno de los desafíos científicos más importantes para el siglo XXI (55). Uno de los caminos propuestos para avanzar en esta compresión es la creación de modelos de redes cerebrales $(1,2)$.

En su más forma más simple, una red es un conjunto de puntos unidos por líneas, o dicho en otros términos, un conjunto de nodos (vértices) unidos por aristas (enlaces o bordes) $(56,57)$. Dado que el cerebro realiza sus funciones integrando la actividad de diversas regiones interconectadas (58), se ajusta a la perfección a este concepto. Así, la red cerebral está constituida por numerosos elementos (neuronas/regiones cerebrales) que para comunicarse por medio de impulsos nerviosos establecen una gran cantidad enlaces (sinapsis/axones) (Fig. 1) $(59,60)$.

Dentro de la noción de red cerebral se engloban distintos tipos de redes. Por ejemplo, los estudios de la organización funcional del cerebro humano basados en la activación de regiones interconectadas - tanto en condición de reposo como durante una actividad específica-, han modelado diversas redes funcionales intrínsecas $(61,62)$, tales como la red 
por defecto o de modo predeterminado (DMN, default mode network), la red frontoparietal, la red visual, la red sensoriomotora, etc. Estas, sin embargo, no son redes totalmente independientes, sino interrelacionadas e integradas en una red general o global, lo que convierte a la red cerebral en una red de redes (63).

La organización de la red cerebral depende de factores genéticos y ambientales, lo cual implica que, si bien preserva sus propiedades fundamentales, puede experimentar cambios continuamente (64). En este sentido, la red es susceptible de sufrir alteraciones que conlleven a una estructura o funcionamiento anormal, como las causadas por diversas enfermedades neuropsiquiátricas. En consecuencia, el estudio de estas enfermedades, y en particular de los trastornos psicóticos, se ha ampliado con un enfoque cimentado en la disfunción de la conectividad de la red cerebral, y han pasado a considerarse como síndromes de desconexión (7).

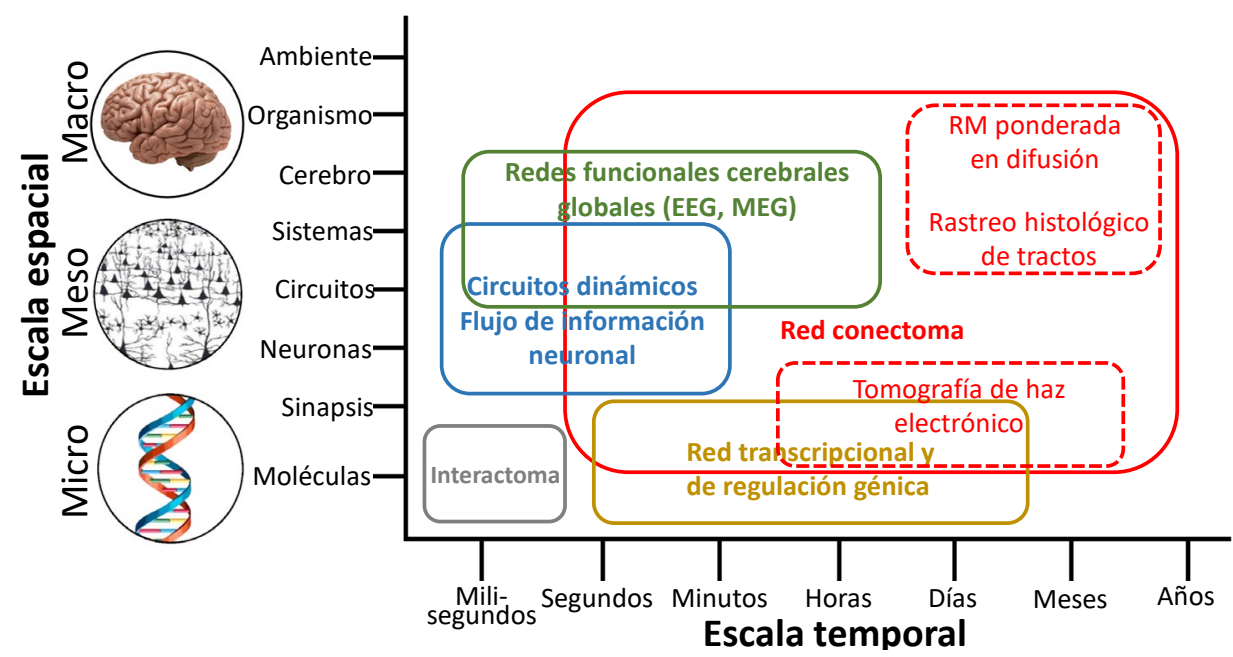

Fig. 1| Redes cerebrales en las escalas temporal y espacial. Según los métodos usados para su elaboración, las redes cerebrales tienen diferentes dimensiones espaciotemporales $(39,58,65,66)$. En la escala temporal, la duración de las redes va desde pocos segundos hasta varios años y pueden ser estáticas o dinámicas según su estabilidad o variación en el tiempo. En la escala espacial, se pueden establecer en la micro- o pequeña escala, (basadas en información codificada en genes o la comunicación a nivel neuronal), en la meso- o escala media (asambleas neuronales, circuitos o redes funcionales intrínsecas) y en la macro- o gran escala (cerebro completo). Se puede ir incluso más allá del propio cerebro para estudiar las interacciones cerebro-ambiente y las interacciones sociales que forman el entramado de las ecologías y culturas. EEG, electroencefalograma; MEG, magnetoencefografía, RM resonancia magnética. Basado en ref. $(39,67)$. 


\subsubsection{Conectividad cerebral estructural y funcional}

La conectividad de la red cerebral es la capacidad de sus elementos integrantes para establecer y mantener conexiones entre sí. En función de los datos con los que se construye la red cerebral, esta conectividad puede ser de tipo estructural, funcional o efectivo $(68,69)$. En esta tesis, el análisis de la red cerebral hace a través de parámetros que examinan la conectividad estructural y funcional.

La conectividad estructural o anatómica se determina en una red en la que sus nodos equivalen a regiones cerebrales concretas (corticales y subcorticales) y sus conexiones o aristas corresponden a los tractos de fibras nerviosas que las conectan, identificados in vivo por técnicas de neuroimagen. Sin embargo, el conocimiento de la organización anatómica o estructural de la red de manera exclusiva no es suficiente para comprender los múltiples, complejos y variables mecanismos operativos que surgen de ella, de ahí la importancia de estudiar también su organización funcional (10).

La conectividad funcional se determina en redes en las que los nodos equivalen a las áreas en las que los sensores registran la actividad cerebral (por técnicas neurofisiológicas) o a determinadas regiones cerebrales (por técnicas de neuroimagen funcional) y las conexiones equivalen a la relación estadística entre las series temporales de la actividad registrada en estas áreas o regiones. Si se especifica la dirección causal de las conexiones entre los elementos de la red, es decir, cómo un elemento influye en otro, se denomina conectividad efectiva $(70,71)$.

Los estudios experimentales y computacionales sugieren la interdependencia en la organización de redes cerebrales estructurales y funcionales: las propiedades dinámicas de las redes funcionales están fuertemente determinadas por las características de la organización estructural, pero también a la inversa, a través de la experiencia y el aprendizaje y en una escala de tiempo más lenta, la organización funcional dinámica puede modular a su vez la estructura anatómica de la red $(72,73)$. 


\subsubsection{Neurociencia de redes: la teoría de redes complejas aplicada al estudio del cerebro}

El cerebro humano es una red compleja, un tipo de red que tiene como característica principal que el conjunto de interacciones entre sus componentes da lugar a propiedades nuevas e inesperadas, llamados fenómenos emergentes $(1,74,75)$. Su estudio por medio de los principios de la teoría de redes complejas - que integra conceptos y herramientas derivados de las matemáticas y la física, como la teoría de grafos y la física estadística $(68,74,76,77)$ - ha abierto una nuevo camino en el estudio de su funcionamiento. Esto ha dado lugar a la neurociencia de redes (network neuroscience) $(67,78)$, que aborda el estudio de la organización estructural y funcional del cerebro con una perspectiva integradora para registrar, modelar y analizar los elementos que lo conforman y sus patrones dinámicos de interacción $(54,65,72,79)$.

El análisis de una red compleja se fundamenta en la estimación de sus características topológicas más importantes $(72,80)$. La topología de una red se refiere a la relación que guarda el conjunto de sus elementos (59), es decir, al patrón de sus conexiones, sin tener en cuenta la distribución de los nodos en el espacio físico. De manera que una red se podría agrandar, encoger o torcer, pero su topología permanecería sin cambios si no se modifican sus conexiones (58).

Ahora bien, para analizar la arquitectura de las redes complejas se han propuesto diferentes tipos modelos y redes. Los principales son el de Erdös-Rényi para redes aleatorias, el de Watts-Strogatz para redes de mundo pequeño y el de Barabási-Albert para redes libres de escala (Fig. 2) $(77,80-82)$.

El modelo de redes de mundo pequeño (SW, small-world) fue introducido en 1998 por D. Watts y S. Strogatz para describir un estado intermedio entre una red regular y una red aleatoria (83). En este modelo la mayor parte de los nodos no están conectados directamente entre sí, sino que forman agrupaciones conectadas de tal forma que para ir desde un nodo dado hasta cualquier otro nodo de la red se requiere un número bajo de conexiones (83). 


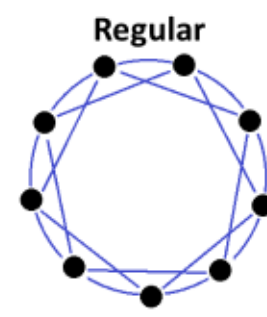

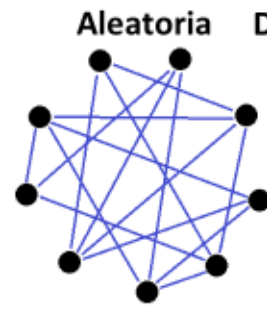

Modelo de

Erlös-Rényi

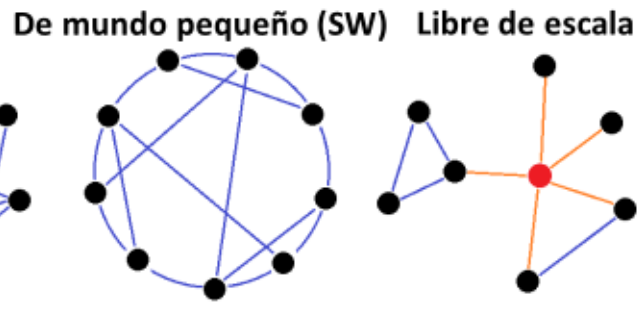

Modelo de

Watts-Strogatz
Modelo de

Barabási-Albert

Fig. 2 Modelos de redes cerebrales. En una red regular los nodos tienen un alto coeficiente de agrupamiento (C), pero una longitud de camino (L) muy alta. Al contrario, las redes aleatorias (84) presentan un bajo $\mathrm{C}$ y una $\mathrm{L}$ baja. El modelo de mundo pequeño (SW) combina el alto $C$ de las redes regulares con la baja $L$ de las redes aleatorias. El modelo libre de escala (85) describe redes que se expanden por la adición continua de nuevos nodos, que se unen a nodos que ya están muy bien conectados Basado en ref. (86).

Aunque en la actualidad existe cierta controversia conceptual y metodológica $(87,88)$, se ha demostrado que el modelo de mundo pequeño está presente en muchas redes reales, incluida la red cerebral en distintas escalas $(89,90)$ y los circuitos corticales funcionales de mamíferos $(90,91)$. Este tipo de red permite una optimización de los procesos de transmisión de información del cerebro (81), facilitando la sincronización entre regiones cerebrales $(92,93)$ y su deterioro se relaciona con el desarrollo de enfermedades cerebrales (94) y con el envejecimiento (95).

\subsubsection{Teoría de grafos: las matemáticas en las redes cerebrales}

En esta tesis la red cerebral se analiza con parámetros de la teoría de grafos, un subdominio de las matemáticas cuyos inicios suelen ubicarse en 1736, cuando L. Euler resolvió el problema de establecer un camino que cruzara una sola vez cada uno de los siete puentes de la antigua ciudad prusiana de Königsberg y volviera al punto de partida, y demostró que tal camino no existe usando un modelo abstracto o grafo (72).

Los grafos son objetos matemáticos constituidos por un conjunto de nodos $V$ (vértices o puntos) conectados por medio de interacciones por pares llamadas aristas $E$ (enlaces, bordes, arcos o líneas del grafo) (96). De esta manera, los grafos cerebrales son una representación matemática del 
conectoma humano (97) en la que el cerebro se convierte en un grupo de nodos interconectados por un conjunto de aristas $(58,98)$.

Existen muchos tipos de grafos. Los construidos en esta investigación fueron no dirigidos y ponderados. En los grafos no dirigidos el orden de los índices en las uniones entre un par de nodos es el mismo, de modo que si $i$ y $j$ son dos nodos de un grafo y $E(i, j)$ representa el enlace que existe entre ellos, en un grafo no dirigido $E(i, j)=E(j, i)$. En los grafos ponderados o pesados cada arista tiene un valor llamado peso o coste numérico que mide o cuantifica ese enlace. Así, $w_{i, j}$ denota el peso asociado a la conexión entre $(i, j)$.

Al elaborar un grafo cerebral es importante tener en cuenta que cada paso del proceso implica tomar decisiones que afectan a los subsecuentes y por tanto al resultado final del análisis $(98,99)$ (Fig. 3). El primer paso es definir los nodos. A nivel teórico, un nodo es idealmente una parte del sistema en estudio, homogénea y con coherencia interna, que se puede separar o delimitar del resto del sistema. Cuanto más similares sean los nodos entre sí, las interacciones entre ellos serán menos significativas $(99,100)$. Los nodos de los grafos estructurales o anatómicos del cerebro pueden obtenerse de la división del cerebro en regiones con cierta homogeneidad (parcelación). En el caso de los grafos funcionales, puede ser adecuado definir como nodo a cada electrodo o sensor que registra la actividad cerebral en una región concreta del cerebro.

A continuación, los siguientes elementos a definir son las aristas, para lo cual se determina algún tipo de unión o relación entre los nodos. En el caso de las redes estructurales las aristas equivalen a las streamlines que conectan dos regiones cerebrales entre sí. Las streamlines son reconstrucciones tridimensionales que indican la trayectoria de los tractos de sustancia blanca estimadas a partir de datos del tensor de difusión (ver sección 1.4.1), y su peso se puede calcular a través de medidas de la difusión del agua, como la anisotropía fraccional promedio. En el caso de las redes funcionales basadas en EEG las aristas ponderadas equivalen a una estimación de la correlación temporal de la señal registrada entre un par de electrodos de registro, que puede establecerse a través de medidas lineares, como la coherencia espectral, o no lineares, como las basadas en sincronización de fase, entre las que se encuentra el valor de acoplamiento de fase (PLV, phase-locking value), usado en esta investigación. 
A

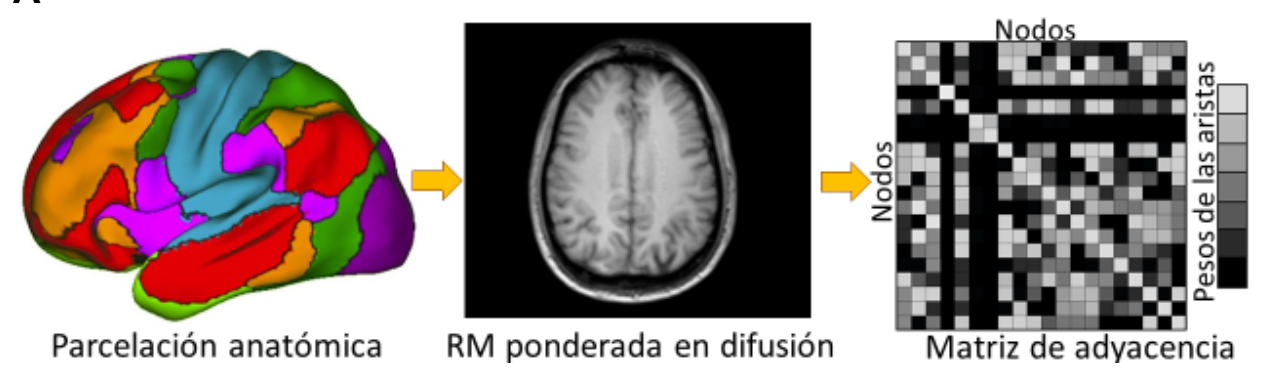

B

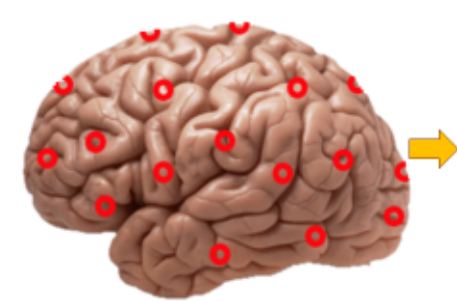

Electrodos de registro

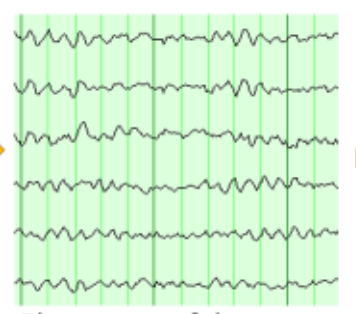

Electroencefalograma

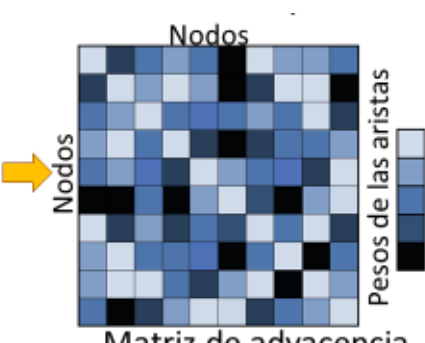

Matriz de adyacencia

Fig. 3| Elaboración de los grafos cerebrales. (A) En esta tesis los grafos de la red estructural se elaboraron definiendo los nodos como las regiones resultantes de la parcelación del cerebro y las aristas como las uniones entre esas regiones determinadas por medio de la tractografía basada en imágenes por resonancia magnética (RM) ponderada en difusión. El peso de las aristas correspondió con el valor de la anisotropía fraccional, una medida de la direccionalidad de la difusión del agua. (B) Los grafos de la red funcional se construyeron definiendo los nodos como los electrodos del electro-encefalograma y las aristas como la correlación temporal de la actividad eléctrica registrada por ellos. En este caso, el peso de las aristas correspondió con el valor de acoplamiento de fase, una medida de la relación de fase entre las señales. Basado en ref. $(72,98)$.

$\mathrm{Al}$ tener definidos ambos elementos se diseña la matriz de asociación o de conectividad, una matriz cuadrada en la que cada uno de sus elementos define el valor de la arista entre un par de nodos por medio del peso de esa conexión. Gracias a esta matriz se puede representar de forma sencilla la red cerebral en cualquier momento determinado, y es posible realizar el cálculo de los parámetros de la teoría de grafos que caracterizan la estructura o la dinámica de la red.

La aplicación de los parámetros de la teoría de grafos para el estudio de los patrones de conectividad de las redes cerebrales está respaldado por una gran cantidad de investigaciones $(94,101-103)$. Estos parámetros son numerosos y están agrupados en distintas categorías, como las medidas de segregación, de integración o de la importancia de los nodos (Fig. 4) (74). 
Estos parámetros se pueden calcular para describir las propiedades de los nodos individuales (parámetros de nodos) y también se pueden estimar para caracterizar en una sola medida a los todos los nodos del grafo de forma global (parámetros de red) $(2,72,100,104,105)$. Los más relevantes para esta investigación son los siguientes:

Grado (degree). En un grafo no dirigido el grado de un nodo $i\left(k_{i}\right)$ se define como el número total de aristas que lo conectan directamente con otros nodos, denominados nodos vecinos. Así, los nodos muy conectados con otros tienen un grado alto. La distribución de probabilidad del grado de todos los nodos la red se denomina distribución de grado (64).

Fuerza (strength). Es una medida de los grafos ponderados que deriva del grado (100). La fuerza de un nodo $i\left(s_{i}\right)$ se define como la suma de los pesos de las aristas que lo conectan con otros nodos. Esta medida proporciona un indicador de la centralidad del nodo (101), que refleja la intensidad de sus conexiones con el resto del grafo. La fuerza de conectividad, CS (connectivity strength) —o densidad — corresponde a la media de la fuerza de todos nodos del grafo y se calcula como la media de los pesos $w_{i, j}$ de todas las aristas del grafo. Esta medida es útil para estimar tanto la complejidad del grafo como su conectividad (106) y como no depende de la distribución de grado, no requiere normalización para su comparación en redes con el mismo número de nodos.
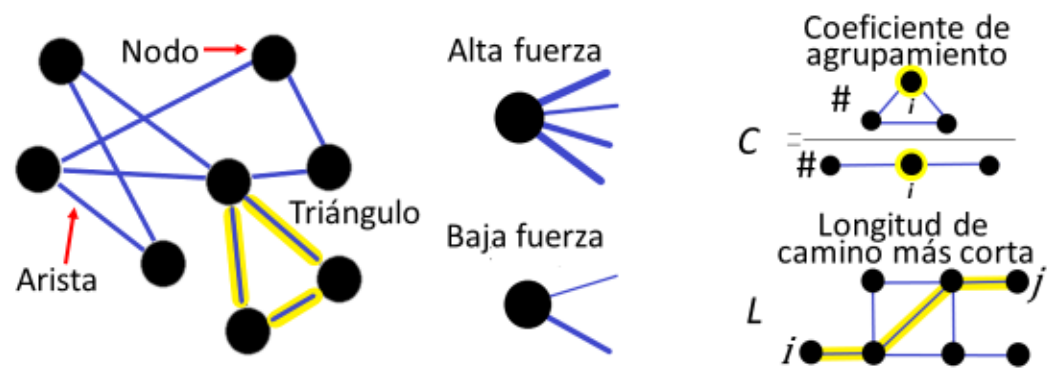

Fig. 4 | Parámetros de la teoría de grafos. La fuerza de un nodo es una medida de la intensidad con la que está unido a la red, estimada a partir del peso de sus aristas. El coeficiente de agrupamiento de un nodo $(C)$ se define como la relación entre el número de triángulos que forma un nodo con sus vecinos y todos los triángulos que podría formar, es decir, el número de triángulos que tiene un nodo $i$ dividido entre el número de tripletes en los que $i$ es el nodo central. La longitud de camino más corta $(L)$ es el número más bajo de aristas que hay que recorrer para pasar de un nodo $i$ a otro $j$. Basado en ref. $(64,107)$ 
Coeficiente de agrupamiento, $\mathbf{C}$ (clustering coeficient). Cuando dos nodos vecinos de un nodo determinado también se conectan directamente entre sí, forman un triángulo (clúster). El coeficiente de agrupamiento de un nodo $i\left(C_{i}\right)$ es el cociente que resulta de dividir el número de triángulos reales de ese nodo entre el número total de triángulos que podría formar con sus nodos vecinos. En otras palabras, el coeficiente de agrupamiento equivale a la fracción de los vecinos de un nodo que también son vecinos entre sí (80), de tal manera que si todos los nodos a los que está conectado un nodo $i$ estuvieran también conectados entre sí, el valor de su $C_{i}$ sería 1. Esta es una medida de segregación o conectividad local de un nodo que proporciona información sobre la intensidad con la que el nodo está conectado localmente en el grafo. El coeficiente de agrupamiento ponderado de un nodo $\left(C_{i}{ }^{w}\right.$, weighted clustering) es el valor del coeficiente de agrupamiento tomando en cuenta los pesos de las aristas. De la media de los coeficientes de agrupación de todos los nodos del grafo (100) se obtiene el coeficiente de agrupamiento de la red, que se relaciona con la eficiencia local en la transferencia de información y con la robustez del grafo frente a los ataques o agresiones externas.

Longitud de camino más corta, $L$ (shortest/minimun path lenght). Para un par de nodos $(i, j)$ un camino (path) es la secuencia ordenada de las aristas que hay que recorrer para pasar del nodo $i$ al $j$, sin pasar por un nodo intermedio más de una vez (103). La longitud de camino más corta en un grafo no ponderado es el camino para pasar de un nodo $i$ a otro $j$, con el menor número posible de aristas y en el caso de un grafo ponderado, es el camino formado por las aristas cuya suma de pesos sea mínima, aunque no sea necesariamente el camino con menos aristas (64). La longitud de camino característica (characteristic path length) se define como la media de los valores de longitud de camino más corta de todos los pares de nodos de la red. Esta es una medida integración o de la conectividad global y tiene una relación inversa con la eficiencia global de la red para la transferencia de la información entre distintas regiones o subsistemas de la red $(64,100)$.

Índice de mundo pequeño, SW (small-worldness). Es un parámetro útil para caracterizar a un grafo en su totalidad, que consiste en la relación entre el coeficiente de agrupamiento $(C)$ y la longitud de camino característica $(L)$, como índices de la conectividad local y de largo 
alcance, respectivamente (100). En una red de mundo pequeño este cociente tendrá un valor más alto que en otro tipo de redes, con un coeficiente de agrupamiento mayor que el de un grafo aleatorio comparable $\left(C^{\text {random}}\right)$, pero con una longitud de camino característica $\left(L^{\text {random }}\right)$ similar $(83,108,109)$.

\subsection{Oscilaciones cerebrales: conexión neuronal a través de la actividad oscilatoria rítmica}

La actividad eléctrica cerebral registrada con electrodos colocados en el cuero cabelludo se representa como fluctuaciones de voltaje en forma de ondas u oscilaciones de apariencia irregular y cambiante. El estudio de las oscilaciones cerebrales es importante en el campo de la conectividad funcional, ya que son el principal mecanismo para transmitir la información e integrar la actividad de los grupos de neuronas, un requisito primordial del funcionamiento cerebral, tanto a nivel cognitivo como motor o sensitivo (110-114). En esta tesis se estudia la red cerebral funcional a partir de la actividad oscilatoria registrada en la banda global $(1-70 \mathrm{~Hz})$ y en un rango de frecuencia específico relacionado con la coordinación neuronal de larga distancia, la banda $\theta(4-8 \mathrm{~Hz})$. Asimismo, se hace un estudio de la potencia de ruido de la actividad oscilatoria en la banda $\gamma(35-45 \mathrm{~Hz})$, un rango de frecuencia relacionado con la conexión de asambleas neuronales corticales a nivel local.

El fenómeno oscilatorio o rítmico es una característica omnipresente en el universo, que se encuentra en el movimiento de las galaxias en un extremo y en el de las partículas subatómicas en otro (115). Los diferentes tipos de registro de la actividad eléctrica del cerebro humano revelan que esta también presenta patrones oscilatorios rítmicos (116).

El término oscilaciones cerebrales (neuronales o neurales), que en ocasiones se usa como sinónimo de ritmos cerebrales, se refiere a los patrones de fluctuación rítmica de la actividad o excitabilidad eléctrica de un conjunto de neuronas a lo largo del tiempo, generados de forma espontánea o en respuesta a estímulos $(16,110)$. Los estudios en los que se ha registrado de manera simultánea el EEG de superficie, los potenciales de campo local y la actividad de las membranas de neuronas, han mostrado que sus oscilaciones son fundamentalmente similares $(117,118)$. 
Las oscilaciones cerebrales reflejan en esencia un patrón de despolarización e hiperpolarización de un gran número de neuronas con interconexiones locales y distantes, que resulta de periodos alternantes de excitabilidad reducida y aumentada dependientes principalmente de la inhibición rítmica de las neuronas excitadoras $(110,119)$. Aunque el origen de la actividad rítmica cortical no se comprende por completo, parece estar mediado por al menos dos mecanismos principales. El primero involucra la interacción entre el tálamo y la corteza, en el cual la actividad de las células marcapasos del tálamo conduce a una activación cortical rítmica. El segundo, por su parte, se relaciona con la capacidad intrínseca de ritmicidad que tienen los grandes circuitos neuronales corticales (120).

La conectividad cerebral funcional se puede determinar mediante estimaciones de la sincronía de la actividad oscilatoria registrada en diferentes regiones (121-123), puesto que, como se ha dicho antes, las oscilaciones rítmicas a distintas frecuencias son el mecanismo para coordinar de forma precisa en el tiempo la actividad de las asambleas neuronales.

La amplitud y duración de las oscilaciones se relaciona con la sincronización de la actividad eléctrica y con el tamaño de los grupos neuronales que las generan. Cuanto mayor sea el número de neuronas y su actividad (excitabilidad) esté más sincronizada, mayor será la suma de su actividad eléctrica, y producirá oscilaciones de gran amplitud y duración (Fig. 5). A la inversa, una menor participación de neuronas o menor sincronía de su actividad implica una oscilación de menor amplitud y duración (124). También hay una relación inversa entre la distancia espacial de las regiones sincronizadas y la frecuencia de las oscilaciones $(125,126)$ de manera que la sincronización de la activación neuronal a corta distancia generalmente involucra frecuencias altas de baja amplitud, mientras que las oscilaciones de frecuencia más baja y mayor amplitud son un fenómeno con más extensión en el cerebro y median la sincronización a larga distancia (113).

La sincronía neuronal cambia a lo largo de la vida, y depende, entre otros factores, de la maduración del cerebro (cambios en la mielinización de las conexiones corticocorticales y el desarrollo de la neurotransmisión gabaérgica) y de los cambios en la frecuencia, amplitud y sincronización de las oscilaciones neuronales $(24,25)$. Pero también la sincronía o en el 
acoplamiento de las oscilaciones neuronales puede alterarse en algunos trastornos mentales, como la esquizofrenia y el trastorno bipolar $(16,25,110,116)$, que se relaciona con patrones de conectividad anómalos, asociados con alteraciones cognitivas y síntomas (23).

\subsubsection{Osciladores cerebrales y bandas de frecuencia}

La actividad oscilatoria del cerebro humano abarca un rango de frecuencias que en general se establece, con unos límites un tanto variables, entre 0.05 y $600 \mathrm{~Hz}$ (127). Su clasificación inició con las primeras descripciones realizadas por H. Berger (128) y fue ampliándose hasta que en 1974 la Federación Internacional de Sociedades de Electroencefalografía y Neurofisiología Clínica propuso las primeras definiciones por consenso de las bandas de frecuencias «clásicas» (129). Esta clasificación ha resultado ser práctica y, con algunas modificaciones, se mantiene en uso en la actualidad. Pero debido a que ese momento los mecanismos y los patrones oscilatorios eran en gran medida desconocidos, la división se hizo siguiendo criterios históricos un tanto arbitrarios, o según lo expresa G. Buzsáki «como las fronteras en línea recta entre las naciones africanas trazadas por los países colonialistas» (27).

El espectro de frecuencias se ha dividido tradicionalmente en rangos o bandas de frecuencia denominados por letras griegas minúsculas, siguiendo la tradición iniciada por $\mathrm{H}$. Berger. Ordenadas de menor a mayor frecuencia son: $\delta(0.1-<4 \mathrm{~Hz}), \theta(4-<8 \mathrm{~Hz}), \alpha(8-13 \mathrm{~Hz}), \beta(>13-30$ $\mathrm{Hz})$ y $\gamma(>30 \mathrm{~Hz})(130,131)$. Se considera que las oscilaciones en determinados rangos de frecuencia resultan de diferentes mecanismos o soportes biológicos. Así, el espectro de frecuencias se relaciona con la actividad de diversos osciladores cerebrales, y por otra parte, los distintos estados fisiológicos y diversas funciones cognitivas concretas se relacionan con la presencia o predominio de la actividad en una u otra banda de frecuencia (27). En esta tesis los dos rangos específicos de actividad oscilatoria que se analizan son las bandas $\theta$ y $\gamma$.

La actividad $\theta$ ha sido bien estudiada en los mamíferos, y se ha registrado en el hipocampo - donde se generan como resultado de la interacción entre neuronas glutamatérgicas, gabaérgicas y colinérgicas (132) - , y en la neocorteza humana. Esta actividad parece desempeñar un 
papel importante en organizar la interacción a larga distancia de múltiples áreas corticales y en una amplia variedad de funciones cognitivas, en especial, la memoria (132-135).

La actividad $\gamma$ es fundamental en el procesamiento cerebral de la información (136-138). Se origina de la interacción o equilibrio excitador/inhibidor (139-141) entre las neuronas piramidales de señalización excitadora glutamatérgica y las interneuronas de disparo rápido que contienen parvalbúmina de señalización inhibidora gabaérgica $(142,143)$, cuya perturbación se asocia con una actividad oscilatoria $\gamma$ anormal que ha sido descrita en varias enfermedades psiquiátricas (144149). Aunque su relevancia funcional sigue sin estar del todo clara (150), las oscilaciones $\gamma$ son muy importantes por varias razones, entre las que están su efectividad para la sincronización de la actividad neuronal $(151,152)$, su participación en la organización de los circuitos o asambleas neuronales locales que se relacionan con las funciones cerebrales superiores - como el mantenimiento y procesamiento atencional $(12,22,23,153)$ y la percepción $(132)$ - y la posibilidad de generarlas por medio de estimulación sensorial o tareas cognitivas (154).

\subsubsection{Sincronización neuronal: conexión por medio de las oscilaciones cerebrales}

Uno de los temas más importantes en el estudio de las oscilaciones es la interacción entre los diferentes ritmos que ocurren simultáneamente. El procesamiento de la información en el cerebro se produce en distintas escalas espaciotemporales de forma simultánea, que se manifiesta en un patrón oscilatorio cortical complejo a distintas frecuencias $(60,155)$. Precisamente, se considera que la comunicación o conexión entre las asambleas neuronales se fundamenta en algún tipo de relación o sincronización flexible de su actividad oscilatoria neuronal $(12,153,156)$.

En general, el acoplamiento o sincronización entre dos ritmos oscilatorios provoca que experimenten algún tipo de modificación. Existe evidencia sugestiva de una jerarquía según la cual las oscilaciones de frecuencia más baja controlan (modelan o modulan) la fase y la amplitud de las oscilaciones de frecuencia más rápida. Por ejemplo, la fase de la oscilación $\theta$ a menudo modela la amplitud de la oscilación $\gamma$ (142) (Fig. 5- 
C). Incluso se ha propuesto que las oscilaciones cerebrales podrían estar alineadas con el ritmo de las oscilaciones de otros sistemas corporales (157).

La sincronización o acoplamiento entre distintos ritmos se puede estimar en base a las propiedades espectrales fundamentales de las oscilaciones, es decir, amplitud, frecuencia y fase (Fig. 5-A) (158). En esta tesis se examina la sincronización basada en la fase. Se considera que hay sincronía de fase entre dos ritmos distintos cuando estos tienen una diferencia de la fase cercana a cero. Esta sincronización reflejaría un momento puntual de comunicación entre los grupos neuronales que generan esos ritmos. Hay diferentes índices de sincronización de fase, entre los que están el índice de retraso de fase (PLI, phase lag index) y el valor de acoplamiento o enganche de fase (PLV, phase-locking value) que, como se ha dicho antes, se utiliza en esta tesis.
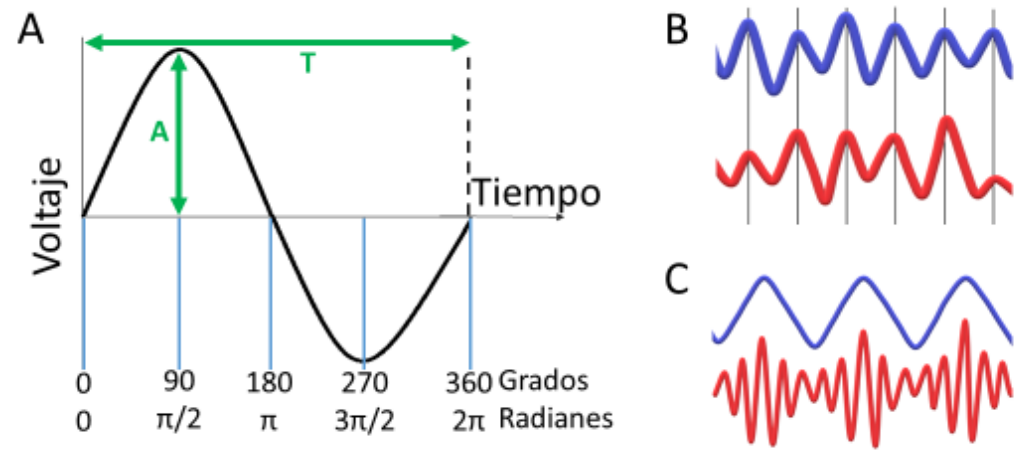

Fig. 5| Parámetros que definen una señal oscilatoria e interacción entre las oscilaciones cerebrales. (A) La frecuencia, $f$, es el número de oscilaciones que ocurren en un segundo. Se mide en hercios $(1 \mathrm{~Hz}=1$ ciclo/s) y se puede calcular a partir valor del período, $T$, como $f=1 / T$. La amplitud, $A$, es el valor máximo de la altura o voltaje de la oscilación y su valor elevado al cuadrado equivale a la potencia. La fase $(\varphi)$ es el ángulo en el tiempo del ciclo oscilatorio, es decir, la posición de la oscilación en un momento a lo largo de su ciclo, y se expresa en grados $\left(0^{\circ}-360^{\circ}\right)$ o en radianes $(0$ - 2) (B-C) Los ritmos de la misma o distinta frecuencia pueden influirse entre sí, modulando su fase y amplitud. En (B) se muestra el acoplamiento fase-fase de dos oscilaciones que pueden tener una amplitud diferente. En (C) se representa el acoplamiento fase-amplitud, por medio del cual una señal lenta modula la amplitud de una más rápida, que queda "anidada". Basado en $(110,159,160)$. 
Con el PLV es posible estimar hasta qué punto la fase de las oscilaciones provocadas por un estímulo se mantiene más o menos constante entre ensayos (inter trial) a lo largo de ventanas de tiempo limitadas, con independencia de su amplitud $(25,159)$. Su rango de valores es de $0 \leq$ PLV $\leq 1$, en el que un valor igual o cercano a 0 indica que no hay sincronización y el valor de 1 indica una sincronización de fase completa (161).

\subsubsection{Tipos de oscilaciones: actividad espontánea y potenciales relacionados con eventos}

La actividad eléctrica del cerebro humano se puede registrar en diferentes condiciones experimentales. Por un lado estarían las oscilaciones en estado de reposo o independientes de estímulo, que representan la actividad cerebral endógena producida cuando una persona no realiza ninguna tarea $(114,162,163)$. Por otro, las oscilaciones generadas durante la realización de una tarea experimental como efecto o respuesta a un evento o estímulo $(25,113,164)$.

Un cambio en la actividad oscilatoria relacionado con un evento experimental (ERP, event related potential) está enganchado en el tiempo con este evento, pero puede estar o no vinculado a la fase. Las respuestas en las que la fase de la respuesta varía de un ensayo son, en esencia, modulaciones de la actividad EEG, por lo que se denominan respuestas inducidas. Por el contrario, la actividad enganchada en tiempo y fase se denomina actividad evocada, porque al inicio es inexistente y sólo es provocada (o evocada, según la traducción más usada) por el evento experimental (165-167).

En un registro EEG, los ERP ocasionan cambios del voltaje a lo largo del tiempo, que reflejan las respuestas cerebrales (sensoriales, motores, afectivos, cognitivos) a los estímulos experimentales (166). Se representan como deflexiones positivas y negativas sobreimpuestas a la actividad de fondo del EEG (168), con una distribución topográfica que indica el grado de involucramiento de áreas corticales concretas con la tarea en ejecución (169).

Uno de los ERP más usados en la investigación en neurociencias y que ha sido analizado en esta tesis para examinar la actividad cortical tanto en el estudio de la conectividad funcional como en de la potencia de 
ruido - es el potencial P300 o P3, descrito por primera vez por S. Sutton y colaboradores en 1965 (170). Este se puede obtener con un diseño experimental simple, por ejemplo, con el paradigma oddball, del cual existen varias modalidades. La utilizada en esta tesis es la del paradigma de tres estímulos auditivos, en el que se presenta un estímulo diana poco frecuente entre una serie de estímulos estándares frecuentes y estímulos distractores infrecuentes.

En cuanto a su significado, la P300 podría reflejar la inhibición neuronal que ocurre durante la atención, necesaria para promover el almacenamiento de la memoria. Al ser registrada por electrodos de la línea media se caracteriza por una distribución en la cual generalmente la amplitud se vuelve mayor y la latencia más corta desde los electrodos frontales a los parietales. Esta amplitud se interpreta como un reflejo de la población neuronal involucrada, aunque está influida por varios factores, como la modalidad del estímulo, las condiciones de la tarea o la edad del sujeto.

Los términos $\mathrm{P} 3 \mathrm{a}$ y $\mathrm{P} 3 \mathrm{~b}$ denotan distintos componentes del P300. A pesar de que los generadores neuronales no se conocen con exactitud, los datos neurofisiológicos y los estudios en lesiones cerebrales sugieren que estos componentes podrían ser variantes del mismo sistema generador que involucra las regiones frontal, temporal y parietal. El P3a es provocado por estímulos distractores pero novedosos y se relaciona con el proceso de atención, en el que participaría la región frontal y la neurotransmisión dopaminérgica. Mientras que el P3b es provocado por estímulos relevantes $\mathrm{y}$ novedosos y se relaciona con el procesamiento neuronal para actualización del contexto y el almacenaje en la memoria, que involucrarían las regiones temporales y parietales y la neurotransmisión noradrenérgica (170).

\subsubsection{Potencia de ruido cortical (NP Noise power)}

En la investigación del funcionamiento del cerebro humano el interés se centra por lo general en el análisis de la respuesta obtenida durante una tarea cognitiva experimental, es decir el análisis de la señal enganchada en tiempo y fase con los estímulos experimentales (ERP). Sin embargo, la actividad eléctrica registrada durante el tiempo en el que ocurre la 
respuesta a un estímulo se compone no solo de la propia respuesta, sino también de otra actividad no enganchada en tiempo y fase con ese estímulo, que podría estar relacionada con otros procesos cerebrales concurrentes, independientes de la tarea experimental $(110,171,172)$.

En otras palabras, siguiendo un modelo lineal o no aditivo (173), la actividad registrada tras un estímulo diana durante la ejecución de una tarea experimental P300 estaría compuesta por dos elementos: (i) la actividad enganchada en tiempo y fase con el estímulo, denominada señal o respuesta, y (ii) la actividad eléctrica enganchada en el tiempo pero no en fase que sería resultado de un incremento de la variabilidad en las repuestas, junto a cualquier otra actividad cortical sin relación directa con el estímulo que ocurre aparentemente al azar, y que en su conjunto se llama ruido neuronal o cortical (174), diferente del ruido no neuronal o artefacto. El aumento de la variabilidad de las respuestas podría ser debido a un aumento de la fluctuación o jitter de la latencia de los ERP o a una alteración de la sincronización o reajuste de fase (phase resetting) de la actividad oscilatoria en curso inducida por el estímulo $(175,176)$.

Los estudios dirigidos a investigar la magnitud de este ruido neuronal, denominada en lo sucesivo potencia de ruido (NP, noise power), han proporcionado información relevante en la esquizofrenia (176-179). Se han informado valores elevados de la NP en la banda $\gamma$ sobre las regiones frontal y de la línea media asociados con déficit cognitivo y con síntomas clínicos en pacientes con esquizofrenia, lo que sugiere que la presencia de una mayor NP puede estar relacionado con el peor estado cognitivo y clínico de los pacientes con esquizofrenia.

Aunque las bases neurofisiológicas del ruido cortical no se conocen con exactitud, los receptores dopaminérgicos D1 y D2 de las neuronas piramidales en la corteza prefrontal - que están estrechamente relacionados con la trasmisión gabaérgica y glutamatérgica-, participarían en la optimización de la relación señal-ruido de microcircuitos neuronales locales y las alteraciones de su activación podrían relacionarse con el aumento del ruido cortical en la esquizofrenia (180). Asimismo, dado que la generación de la actividad cortical parece depender de un equilibrio de mecanismos de excitación e inhibición neuronales, el ruido neuronal podría estar relacionado con un desequilibrio de este balance de excitación/inhibición. 


\subsection{Instrumentos para la adquisición de los datos de conectividad}

El estudio de la conectividad cerebral se ha realizado a partir de datos estructurales y funcionales. En esta tesis la red estructural se basa en datos de imagen por resonancia magnética ponderada en difusión, que a partir del análisis de la difusión de las moléculas de agua en los tejidos cerebrales (181) permite la reconstrucción y visualización in vivo de los tractos de axones en la sustancia blanca (182). Por otra parte, la electroencefalografía se usa para construir las redes cerebrales funcionales. Con esta técnica — una de las primeras introducidas en el estudio de la fisiología cerebral que aún sigue en uso- se miden los campos eléctricos creados por la actividad neuronal de manera no invasiva, con una resolución temporal muy alta (183).

\subsubsection{Resonancia magnética ponderada en difusión}

La resonancia magnética (RM) es una técnica para generar imágenes basada en las respuestas de los protones de los átomos de hidrógeno al ser sometidos a potentes gradientes de campo magnético y a pulsos de radiofrecuencia $(181,184)$. Cuando estos protones vuelven a su estado inicial - el tiempo de relajación - liberan energía que se registra con una bobina receptora, generándose así la señal de la RM $(185,186)$.

En una imagen de RM convencional el contraste (brillo) depende de la intensidad de la señal en cada uno de los elementos de imagen o píxeles (pixel, picture element), que a su vez se relaciona con la cantidad de moléculas de agua que contienen los tejidos. Debido a que el contenido de agua de los tractos que forman la sustancia blanca es similar, no hay un contraste adecuado entre estas estructuras y resulta muy difícil identificar tractos específicos. Esta limitación se resuelve con el uso de secuencias especiales en las que se miden propiedades locales químicas y físicas del agua, como la difusión y el flujo, para obtener las llamadas imágenes ponderadas o potenciadas en difusión $(184,187)$.

La RM ponderada en difusión (DWI, diffusion-weighted imaging) es una secuencia funcional que se fundamenta en el análisis del fenómeno de difusión de las moléculas de agua dentro de cada vóxel (volumetric pixel), la unidad cúbica tridimensional de la imagen $(188,189)$. Con esta técnica 
se generan imágenes cuyo contraste refleja las diferencias de difusión del agua en los tejidos y permite reconstruir la disposición de los tractos de fibras en la sustancia blanca $(186,190)$. La difusión del agua es el movimiento o desplazamiento microscópico aleatorio de las moléculas de agua debido a la agitación térmica (movimiento browniano) $(181,191)$. Si las moléculas se mueven libremente en todas las direcciones (como en el líquido cefalorraquídeo), la difusión se denomina isotrópica; en cambio, si el movimiento queda obstaculizado en alguna dirección y favorecido en otra (como dentro de un axón/tracto de axones) se llama difusión anisotrópica $(187,192)$ (Fig. 6).

A

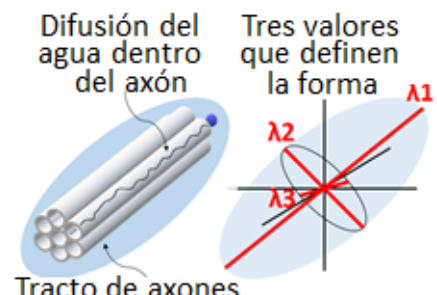

Tracto de axones

C

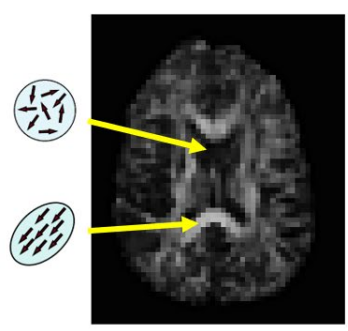

B

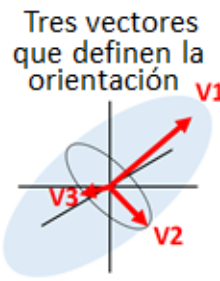

D

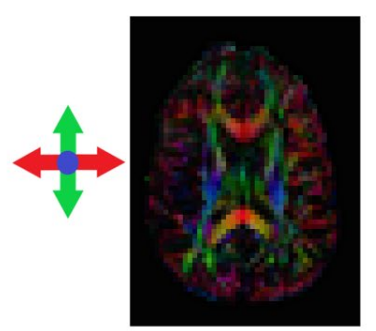

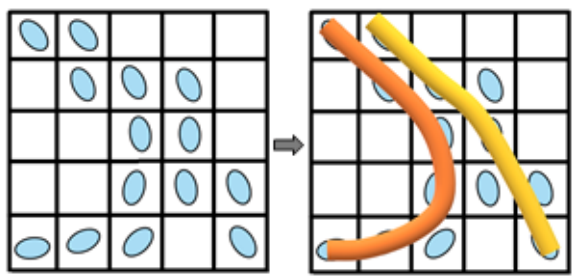

E

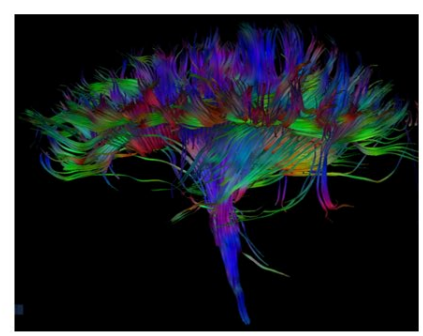

Fig. 6 Tractografía por tensor de difusión. (A) La restricción de la difusión del agua en los axones sirve de base para estimar el elipsoide de difusión por cada vóxel, del cual se determina su forma y orientación. (B) En la tractografía con algoritmos deterministas las streamlines (en color naranja y amarillo) siguen la dirección principal de la difusión del agua vóxel por vóxel, mientras que con algoritmos probabilísticos es posible estimar la incertidumbre de la trayectoria de un tracto. (C) En el mapa de anisotropía fraccional el contraste refleja si la difusión en un píxel es anisotrópica (píxel brillante) o isotrópica (oscuro). (D) En el mapa en colores la dirección de la difusión se representa por un código de colores: verde (anterior-posterior), rojo (izquierda-derecha) y azul (superior-inferior). (E) El tractograma cerebral reconstruye in vivo la disposición anatómica tridimensional de los tractos de sustancia blanca en el cerebro. Basado en ref. $(58,181)$. 


\subsubsection{Elipsoide y tensor de difusión}

La aplicación de un gradiente de campo magnético en una dirección determinada permite medir la difusión del agua en esa dirección (193). Cuando se utilizan al menos 6 direcciones ortogonales, los datos de difusión en cada vóxel se pueden representar mediante un elipsoide tridimensional simétrico (187) (Fig. 6). Este elipsoide describe las características de la difusión del agua, y si se da por hecho que la dirección de las fibras nerviosas coincide con la dirección del movimiento de las moléculas de agua, permite establecer la orientación predominante de los tractos de fibras nerviosas dentro de cada vóxel (194). En este proceso de medición y ajuste se utiliza un tensor $(D) 3 \times 3$, que da nombre a la técnica de imagen, que es llamada RM por tensor de difusión (DTI, diffusion tensor imaging) (195).

La magnitud y dirección del elipsoide de difusión en cada vóxel se determinan a partir de tres valores propios $\lambda$ (eigenvalues) — que definen la forma - y tres vectores propios $v$ (eigenvectors) - que definen la orientación-(Fig. 6A). Cada uno de los valores propios se asocia con un vector propio, ordenados de modo que $\lambda_{1}$ es el valor propio principal que representa la difusión a lo largo de $v_{l}$ (191).

La información de los parámetros del elipsoide de difusión se puede representar de diferentes maneras, como el mapa de anisotropía, el mapa de orientación y el tractograma tridimensional $(181,196)$. Mediante el uso de la DTI no es posible establecer el trazado de un único axón, ya que la estimación de la difusión del agua se realiza por cada vóxel, dentro del cual hay muchos axones. En cambio, se puede representar la trayectoria de un haz o un tracto formado por muchas fibras nerviosas con disposición homogénea y con varios vóxeles de diámetro $(181,187,195)$.

La tractografía estima las trayectorias de los tractos en la sustancia blanca partiendo de los valores propios principales $\left(\lambda_{l}\right)$ de los vóxeles, representándolas con líneas de mayor difusión local llamadas streamlines, que son la representación tridimensional de las trayectorias de los tractos de fibras. En esta tesis para determinar las streamlines se sigue un método probabilístico de seguimiento de fibra, el cual incorpora la incertidumbre esperada en el algoritmo de seguimiento y permite generar varios streamlines dentro de un solo vóxel en regiones de mayor incertidumbre, con la posibilidad de delinear una porción mayor de un tracto de sustancia blanca (197). 


\subsubsection{Anisotropía fraccional}

Los datos del tensor de difusión también son útiles para calcular los parámetros que cuantifican la difusividad local, como la anisotropía fraccional (FA, fractional anisotropy). Esta medida cuantifica el grado de dirección de la difusión de agua en cada vóxel (198) y su valor va de 0 para una difusión completamente isotrópica o esférica en la que todos los valores propios son iguales-, a 1 -para una difusión totalmente anisotrópica o lineal completa en la que solo hay un valor propio $>0$ La FA se calcula a partir los 3 valores propios del elipsoide de difusión $\left(\lambda_{1}, \lambda_{2}, \lambda_{3}\right)$, es decir, los valores de difusión en las 3 direcciones principales que definen la forma del elipsoide y algunos factores, como la densidad axonal, la mielinización y la homogeneidad en la orientación axonal, pueden afectar su estimación $(181,195)$.

\subsubsection{Electroencefalograma}

Desde finales del siglo XIX diferentes estudios electrofisiológicos, como los llevados a cabo por R. Caton, habían demostrado la naturaleza eléctrica de la actividad cerebral en animales (199). Pero fue H. Berger, quien en 1924 abrió un nuevo capítulo en el estudio de la fisiología cerebral cuando realizó el que es considerado como el primer registro de la actividad eléctrica cerebral en humanos, que él llamaría electroencefalograma (EEG) $(128,200,201)$. Desde entonces, el EEG ha sido una herramienta de gran utilidad en el estudio de la fisiología cerebral, con importantes aplicaciones clínicas y en la investigación, que incluye su uso para el estudio de la conectividad funcional (24).

La electroencefalografía es una técnica que mide la actividad neuronal oscilatoria con una alta resolución temporal, por lo que es adecuada para evaluar los cambios rápidos vinculados a la función cognitiva $(23,202)$. Tiene una resolución espacial baja, dependiente del número de electrodos de registro y de la distancia entre ellos, si bien registros de alta densidad con más de 250 sensores, y nuevos métodos de reconstrucción de las fuentes de la actividad eléctrica pueden lograr en la actualidad una resolución espacial cercana a unos pocos milímetros (203). 
A

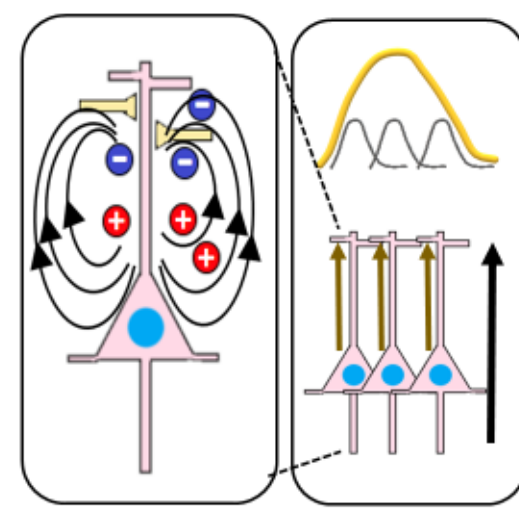

C

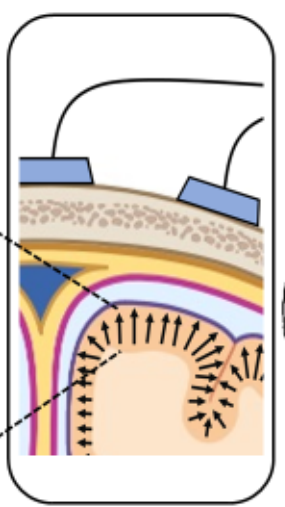

D

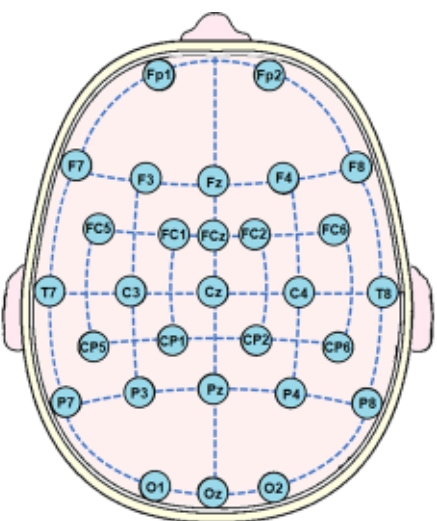

Fig. 7| Electroencefalograma (EEG). (A) Las neuronas piramidales de la corteza experimentan potenciales postsinápticos, los cuales conducen a la formación de un dipolo llamado potencial de campo local $(27,118)$. (B) La actividad sincrónica de un grupo de neuronas piramidales con disposición paralela crea un campo eléctrico de una magnitud suficiente como para extenderse en forma de onda electroquímica por el volumen extracelular y alcanzar la superficie $(118,204,205)$. (C) El EEG es la versión atenuada de los potenciales de campo local a través de tejidos con distinta conductividad eléctrica situados entre la fuente de la señal y el electrodo de registro (206). (D) Localización de los electrodos de registro usados en esta tesis según el sistema internacional 10-20, llamado así porque las distancias entre los electrodos representan un 10 o 20 por 100 de las distancias de los ejes que unen determinados puntos craneométricos. Basado en ref. $(205,207,208)$.

Origen de la señal del EEG y potenciales de campo eléctrico. El EEG es el registro de los campos eléctricos producidos por la actividad neuronal de la corteza cerebral usando electrodos localizados en el cuero cabelludo. Se estima que alrededor de 60 a 100 millones de neuronas - que se encuentran en un área superficial cercana a $6-10 \mathrm{~cm}^{2}$ - deben tener una actividad sincrónica para formar un campo eléctrico dipolar de magnitud suficiente como para ser detectado por un electrodo de registro (204) (Fig. 7). La señal EEG es la representación gráfica en un osciloscopio de la fluctuación de la diferencia del voltaje del potencial eléctrico entre dos electrodos a lo largo del tiempo.

El EEG se origina principalmente en las neuronas piramidales de la corteza que tienen el eje longitudinal del soma y de la dendrita apical dispuesto en forma paralela al de neuronas vecinas y perpendicular a la superficie del electrodo de registro. Se considera que de los distintos tipos de actividad eléctrica que experimentan estas neuronas los potenciales postsinápticos son los responsables de originar la señal del EEG (118) puesto que, aunque de menor voltaje que los potenciales de acción, tienen 
una duración suficiente (50-200 ms) como para permitir su suma espaciotemporal, que hace posible la creación de un campo eléctrico registrable (Fig. 7).

\subsection{Estado de la cuestión}

En esta sección se ofrece una visión general de los antecedentes más relevantes del análisis de la conectividad estructural y funcional y del estudio de la potencia de ruido de la actividad oscilatoria en la banda $\gamma$ realizados en la esquizofrenia y el trastorno bipolar, subdivididos de acuerdo con los tres estudios realizados.

\subsubsection{Conectividad estructural: análisis de la CS y del SW}

Los análisis de la conectividad estructural en la esquizofrenia basados en los parámetros de la teoría de grafos han revelado propiedades de SW alteradas (2) debido a un patrón de integración disminuida (es decir, un valor de L más alto). Otras alteraciones de la conectividad descritas en este síndrome incluyen un aumento del C (21) y una reducción de la eficiencia global (3).

En la revisión bibliográfica realizada no se encontraron estudios que, utilizando parámetros de la teoría de grafos, compararan directamente la conectividad estructural entre pacientes con esquizofrenia y trastorno bipolar a partir de redes cerebrales elaboradas con datos de DTI, si bien otros estudios han comparado la conectividad estructural cerebral entre estos trastornos usando otros métodos. Un estudio comparó la conectividad estructural entre la esquizofrenia y el trastorno bipolar basado en correlaciones de grosor cortical (209); otro evaluó con parámetros de grafos la organización de la red estructural en pacientes con esquizofrenia de primer episodio sin tratamiento previo (210), revelando valores de L más altos pero de SW conservados; y otro estudio basado en datos de resonancia magnética funcional reveló una conectividad tanto aumentada como disminuida a través de diferentes regiones cerebrales en pacientes con esquizofrenia al compararlos con controles, con diferencias intermedias en los pacientes bipolares (211). 
Una investigación reciente mostró reducciones generalizadas de anisotropía fraccional (FA) en la esquizofrenia y el trastorno bipolar, así como una FA más alta en la esquizofrenia en áreas que se corresponden con la red neuronal por defecto (DMN) (212). En otro análisis de conectividad estructural, se describió un $C$ global reducido y una $L$ aumentada en pacientes con trastorno bipolar en comparación con controles sanos (213).

Del mismo modo, los estudios demuestran de que el tratamiento no parece ser responsable de las alteraciones de la conectividad encontradas en estas enfermedades. En un estudio basado en DTI de pacientes con esquizofrenia en el primer episodio sin tratamiento se encontraron alteraciones de la conectividad estructural (214). Asimismo otro estudio de DTI que comparó un grupo de pacientes con esquizofrenia crónica que nunca había recibido tratamiento y otro grupo que sí lo había recibido mostró una mayor reducción de la FA en algunos tractos de sustancia blanca en pacientes no tratados que en los que si recibían tratamiento, demostrando que el tratamiento antipsicótico no influye de forma adversa en las alteraciones de la FA (215).

\subsubsection{Conectividad funcional: análisis de la CS y su modulación durante una tarea cognitiva}

En un estudio que examinó la conectividad funcional en la esquizofrenia a partir de registros EEG utilizando el parámetro SW y su modulación relacionada con una tarea cognitiva $\mathrm{P} 300$, se encontró que, en comparación con los controles, se produce una menor modulación del SW en la esquizofrenia (es decir, un menor aumento del valor este índice durante la realización de la tarea cognitiva), sugestiva de una menor capacidad de integración de la información en este síndrome (18).

En otro estudio de la conectividad funcional utilizando el parámetro de CS — realizado también a partir de registros EEG durante la realización de una tarea cognitiva - se informaron valores de CS basales más altos en pacientes con esquizofrenia en las bandas global y $\theta$ en comparación con controles. De estos, la CS en la banda $\theta$ tuvo una menor modulación durante una tarea P300 (216). Esta menor modulación relacionada con la tarea es un hallazgo que también se ha replicado en otros estudios de las 
propiedades espectrales del EEG en dos muestras diferentes de pacientes con esquizofrenia $(217,218)$.

En un estudio de RM funcional en estado de reposo en pacientes con esquizofrenia sin tratamiento y pacientes crónicos tratados (219) se encontró que ambos grupos de pacientes mostraban alteraciones en parámetros de la teoría de grafos al compararlos con los controles. Sin embargo, los pacientes que nunca habían recibido tratamiento mostraron mayor reducción de la eficacia global en relación con los pacientes tratados con antipsicóticos y controles, lo que indicaría que el tratamiento antipsicótico a largo plazo podría proteger parcialmente o mejorar la conectividad cerebral en la esquizofrenia. Del mismo modo, en otro estudio se sugirió que los fármacos antipsicóticos podrían mejorar algunas anomalías de la red (reducción del coeficiente de agrupamiento) del conectoma funcional en pacientes con esquizofrenia (220).

\subsubsection{Actividad oscilatoria Y: análisis de la NP}

Numerosas investigaciones han mostrado anormalidades en la actividad oscilatoria $\gamma$ en la esquizofrenia, relacionadas con síntomas clínicos y deterioro cognitivo $(23,147,148,221)$ y se ha propuesto que estas también podrían estar relacionadas con la disfunción cognitiva y los síntomas en el trastorno bipolar $(24,222,223)$. Los estudios de la actividad oscilatoria en la banda $\gamma$ basados en la NP en pacientes con esquizofrenia han mostrado valores elevados de este parámetro en la línea media y en regiones frontal o parietal que han sido asociados con déficits cognitivos y síntomas clínicos $(177,179,224)$, aunque esta relación no ha sido encontrada en el trastorno bipolar (177).

Las elevaciones de la NP en la banda $\gamma$ en la esquizofrenia han sido relacionadas con alteraciones cognitivas $(172,224)$ y con síntomas negativos (224) y se han relacionado con la disminución de la variabilidad de la actividad eléctrica cerebral durante una tarea cognitiva (178). 


\section{CAPÍTULO 2}

\section{Hipótesis y objetivos}

ara el correcto funcionamiento del cerebro resultan
imprescindibles unos adecuados patrones de conectividad entre
distintos grupos neuronales localizados en diferentes regiones. Estos patrones pueden estar alterados en algunas enfermedades mentales, incluidos los trastornos psicóticos, de los cuales la esquizofrenia y el trastorno bipolar tipo I son los principales ejemplos.

Por tanto, resulta de interés examinar la conectividad cerebral en estas dos enfermedades con una metodología uniforme para determinar las anormalidades respecto a un grupo de controles sanos, y al mismo tiempo permita reconocer las coincidencias y diferencias entre los dos trastornos. Asimismo, es relevante examinar el papel de la cronicidad y del tratamiento sobre las alteraciones de la conectividad cerebral ya que, si se determinan en pacientes crónicos que reciben tratamiento farmacológico, es posible que estos factores tengan un papel potencial sobre estas alteraciones.

Así pues, en esta tesis se estudia la conectividad cerebral en la esquizofrenia y el trastorno bipolar en redes estructurales y funcionales. Y dado que la conectividad funcional se relaciona con la sincronización de la actividad oscilatoria rítmica de neuronas distribuidas en amplias regiones cerebrales (5), también se hace un estudio de las oscilaciones corticales en la banda $\gamma(35-45 \mathrm{~Hz})$, las cuales sustentan la conectividad de las asambleas neuronales a nivel local. 
Con base en investigaciones realizadas en este campo, la hipótesis principal de esta tesis es que las alteraciones de los diferentes parámetros de la conectividad estructural y funcional y de la sincronización de la actividad oscilatoria en la banda $\gamma$ son mayores en pacientes con esquizofrenia en comparación con pacientes con trastorno bipolar. Del mismo modo, las hipótesis secundarias son (i) que las alteraciones detectadas no se relacionan con el tratamiento con antipsicóticos u otros grupos de psicofármacos, ni con la duración de la enfermedad y (ii) que cuanto mayor es la alteración de los parámetros de conectividad y sincronización, hay más manifestaciones clínicas y peor desempeño en las pruebas cognitivas.

El objetivo general de esta tesis es estimar las alteraciones de los parámetros de conectividad estructural y funcional de la red cerebral y de la sincronización de la actividad neuronal en la banda $\gamma$ en una muestra de pacientes con esquizofrenia y trastorno bipolar, comparándolos con un grupo de controles sanos. En el estudio de la red estructural estos parámetros son la fuerza de conectividad (CS) y el índice de mundo pequeño (SW). En el estudio de la red funcional el parámetro es la CS en las bandas global y $\theta$ durante una tarea cognitiva P300, estimando su valor en la condición preestímulo y su modificación durante la respuesta de la tarea, que en esta tesis se denomina modulación relacionada con la tarea. En el estudio de la actividad en la banda $\gamma$ el parámetro usado es la potencia de ruido cortical (NP), que refleja la magnitud de la actividad oscilatoria que no está vinculado con el estímulo de una tarea cognitiva en ejecución.

Los objetivos específicos son:

a) Analizar la especificidad para el diagnóstico clínico de las alteraciones de la CS y el SW de la red cerebral estructural.

b) Determinar la especificidad para el diagnóstico de las alteraciones de la CS en la red funcional en la condición preestímulo y su modulación relacionada con una tarea cognitiva. 
c) Evaluar la especificidad para el diagnóstico de las alteraciones de la potencia de ruido cortical en la banda $\gamma(\mathrm{NP}-\gamma)$.

d) Estudiar el papel de la duración de la enfermedad en las alteraciones de los parámetros estudiados.

e) Investigar los posibles efectos del tratamiento con antipsicóticos y otros psicofármacos en estas alteraciones.

f) Examinar la relación de estas alteraciones con los síntomas clínicos y la cognición. 



\section{CAPÍTULO 3}

\section{Metodología}

$\mathrm{n}$ este capítulo se describen de manera general los materiales y métodos utilizados en los tres estudios que conforman esta tesis. En cada uno de ellos se adaptó la metodología aquí descrita, según los requerimientos de su diseño (Tabla 1), y puede consultarse en la respectiva sección de cada publicación en los Anexos.

\subsection{Participantes}

Los participantes se dividieron en tres grupos: (i) pacientes con esquizofrenia (ESQ) - en el estudio de la conectividad estructural se subdividió en pacientes con esquizofrenia en el primer episodio (EPE) y crónica (EC)—, (ii) pacientes con trastorno bipolar I en eutimia (TB) y (iii) controles sanos (CTRL). Su número varió en los artículos, según los datos disponibles.

El diagnóstico se realizó a través de entrevistas clínicas a los pacientes y sus familiares, siguiendo los criterios del Manual diagnóstico y estadístico de los trastornos mentales en su quinta edición (DSM-5) (45). Los criterios de exclusión fueron: (i) cualquier enfermedad neurológica, (ii) antecedentes de traumatismo craneoencefálico con pérdida de conciencia de más de un minuto, (iii) abuso de sustancias pasado o presente - excepto nicotina o cafeína-, (iv) cociente intelectual total menor de 70, (iv) cualquier otro trastorno mental en el caso de los pacientes y (v) en el caso los controles, cualquier otra 
condición psiquiátrica o neurológica en tratamiento con psicofármacos. Además, todos los participantes negaron problemas de audición.

\section{Tabla 1 | Aspectos metodológicos de los artículos de investigación}

\begin{tabular}{|c|c|c|c|}
\hline Publicación & Participantes & Psicometría & $\begin{array}{c}\text { Parámetros de } \\
\text { estudio }\end{array}$ \\
\hline $\begin{array}{l}\text { Artículo 1: } \\
\text { Conectividad } \\
\text { estructural en la } \\
\text { esquizofrenia y el } \\
\text { trastorno bipolar: } \\
\text { efectos de la cronicidad } \\
\text { y el tratamiento } \\
\text { antipsicótico }\end{array}$ & $\begin{array}{l}18 \mathrm{EPE} \\
25 \mathrm{EC} \\
24 \mathrm{~TB} \\
28 \mathrm{CTRL}\end{array}$ & $\begin{array}{l}\text { PANSS } \\
\text { BNSS } \\
\text { WAIS-III } \\
\text { WCST } \\
\text { BACS } \\
\text { MSCEIT }\end{array}$ & $\begin{array}{l}\text { CS } \\
\text { SW }\end{array}$ \\
\hline $\begin{array}{l}\text { Artículo 2: } \\
\text { Fuerza de conectividad } \\
\text { de la red funcional } \\
\text { basada en EEG en la } \\
\text { esquizofrenia y el } \\
\text { trastorno bipolar }\end{array}$ & $\begin{array}{l}79 \mathrm{ESQ} \\
\text { (31 EPE } \\
\text { y } 48 \mathrm{EC}) \\
29 \mathrm{~TB} \\
63 \mathrm{CTRL}\end{array}$ & $\begin{array}{l}\text { PANSS } \\
\text { HDRS } \\
\text { YMRS } \\
\text { WAIS-III } \\
\text { BACS }\end{array}$ & $\begin{array}{l}\text {-CS en la ventana } \\
\text { preestímulo } \\
\text {-Modulación de la } \\
\text { CS relacionada } \\
\text { con la tarea } \\
\text { (ambas en las } \\
\text { bandas global y } \theta \text { ) }\end{array}$ \\
\hline $\begin{array}{l}\text { Artículo 3: } \\
\text { Potencia de ruido en la } \\
\text { banda y en la } \\
\text { esquizofrenia y el } \\
\text { trastorno bipolar } \\
\text { durante una tarea } \\
\text { cognitiva }\end{array}$ & $\begin{array}{l}29 \text { ESQ } \\
27 \text { TB } \\
36 \text { CTRL }\end{array}$ & $\begin{array}{l}\text { PANSS } \\
\text { WAIS-III } \\
\text { BACS }\end{array}$ & $N P-\gamma$ \\
\hline
\end{tabular}

EPE, pacientes con esquizofrenia en el primer episodio; EC, pacientes con esquizofrenia crónica; $E S Q$, pacientes con esquizofrenia; TB, pacientes con trastorno bipolar I; CTRL, controles sanos; PANSS, Escala para el síndrome positivo y negativo de la esquizofrenia; BNSS, Escala breve de síntomas negativos; WAIS-III, Escala de inteligencia para adultos de Wechsler- tercera edición; WCST, Test de clasificación de tarjetas de Wisconsin; BACS, Evaluación breve de la cognición en esquizofrenia; MSCEIT, Prueba de inteligencia emocional Mayer-Salovey-Caruso; HDRS, Escala de evaluación para la depresión de Hamilton; YMRS, Escala de manía de Young CS, fuerza de conectividad; SW, índice de mundo pequeño; NP-y, potencia de ruido en la banda $\mathrm{y}$. 
Asimismo, todos los participantes expresaron por escrito su consentimiento para participar en la investigación después de haberles proporcionado la información completa impresa y verbal sobre los procedimientos para la adquisición de los datos y su uso. El comité de ética local del Hospital Clínico Universitario de Valladolid aprobó las investigaciones, de acuerdo con el Código de Ética de la Asociación Médica Mundial (Declaración de Helsinki).

\subsection{Valoración de la cognición, de los síntomas y del tratamiento farmacológico}

Para la evaluación cognitiva de todos los participantes se aplicaron los siguientes instrumentos en su versión en español:

a) Escala de inteligencia para adultos de Wechsler WAIS-III (225).

b) Prueba de clasificación de tarjetas de Wisconsin (WCST) (226).

c) Escala de evaluación breve en cognición en la esquizofrenia (BACS) (227).

d) Test de inteligencia emocional Mayer-Salovey-Caruso (MSCEIT) (228).

En la evaluación de los síntomas en los pacientes se utilizaron las siguientes escalas:

a) Escala del síndrome positivo y negativo (PANSS) (229).

b) Escala breve de síntomas negativos (BNSS) (230).

c) Escala para la evaluación de la depresión de Hamilton (HRDS) (231).

d) Escala de manía de Young (YMRS) (232).

E1 tratamiento farmacológico con antipsicóticos, litio, benzodiacepinas, anticonvulsivos y/o antidepresivos fue informado por el psiquiatra a cargo del paciente, y se mantuvo sin cambios los 3 meses previos a la realización del estudio. La dosis del tratamiento antipsicótico se transformó en equivalentes de clorpromazina (expresados en $\mathrm{mg} / \mathrm{d}$ ). Asimismo, se comprobó la adherencia al tratamiento, considerándola adecuada si pacientes retiraron más del $70 \%$ de las recetas de las oficinas de farmacia durante los seis meses previos a su inclusión en la investigación. 


\subsection{Adquisición y procesamiento de las imágenes por resonancia magnética ponderada en difusión}

La adquisición de las imágenes se realizó con un sistema Achieva $3 \mathrm{~T}$ (Philips Healthcare; Best, Países Bajos) y el procesamiento fue realizado utilizando los programas informáticos gratuitos FSL, Freesurfer y MRtrix (233-235). El protocolo de adquisición duró 18 minutos, e incluyó imágenes anatómicas ponderadas en T1 e imágenes ponderadas en difusión. Sus principales aspectos técnicos se detallan a continuación.

Para las imágenes anatómicas ponderadas en T1 se empleó una secuencia TFE (turbo field echo), el tamaño del vóxel fue de $1 \times 1 \times 1$ $\mathrm{mm}^{3}$ y el de la matriz fue de 256 x 256, adquiriéndose 160 cortes sagitales que abarcaron la totalidad del cerebro. Para las imágenes ponderadas en difusión se empleó una secuencia EPI (echo-planar imaging) para obtener 61 imágenes con diferentes direcciones del gradiente de difusión (b-valor $=1000 \mathrm{~s} / \mathrm{mm}^{2}$ ) y una imagen basal (bvalor $=0 \mathrm{~s} / \mathrm{mm}^{2}$ ). El tamaño del vóxel fue de $2 \times 2 \times 2 \mathrm{~mm}^{3}$ y el de la matriz fue de $128 \times 128$, adquiriéndose 66 cortes axiales.

Con las imágenes ponderadas en T1 se realizó una parcelación cortical y una segmentación de estructuras de sustancia gris subcortical usando el atlas de Desikan-Killiany (236). Se obtuvieron 84 regiones en total, que por cada lado incluían 34 regiones cerebrales corticales, 7 regiones cerebrales subcorticales y 1 región del cerebelo.

Esta información se combinó con las imágenes ponderadas en difusión para obtener una tractografía con restricción anatómica (ACT, anatomically-constrained tractography) del cerebro completo. Se generaron 2 millones de streamlines por cada participante y se crearon los mapas de anisotropía fraccional (FA, fractional anisotropy).

A partir de los resultados de la parcelación cortical y la tractografía se calculó el conectoma, construyendo una matriz de adyacencia (o de conectividad) simétrica $84 \times 84$ por cada participante. Los nodos de la red estructural que componen la matriz se corresponden con las regiones resultantes de la parcelación. Las aristas equivalen a las streamlines entre cada par de esas regiones. El peso de cada arista resulta del valor de la FA promedio a lo largo de las streamlines que contiene. No se aplicó ningún umbral a las matrices, por lo que los coeficientes de la matriz 
pudieron ser iguales a cero en caso de no reconstruirse streamlines entre dos regiones.

\subsection{Adquisición de los electroencefalogramas durante un paradigma oddball}

Para la adquisición de los EEG se utilizó un equipo Brain Vision ${ }^{\circledR}$ (Brain Products $\mathrm{GmbH}$; Múnich, Alemania) de 29 electrodos colocados en un gorro elástico (Electro-Cap International, Inc.; Eaton, Ohio, EE. UU.), de acuerdo con el Sistema internacional 10/20 modificado, que fueron: Fp1, Fp2, F7, F3, Fz, F4, F8, FC5, FC1, FCZ, FC2, FC6, T7, C3, Cz, C4, T8, CP5, CP1, CP2, CP6, P7, P3, Pz, P4, P8, O1, Oz y O2. Además, se colocaron dos electrodos adicionales de electrooculografía (EOG) para registrar los movimientos de los parpados y ojos.

La impedancia de los electrodos se mantuvo por debajo de $5 \mathrm{k} \Omega$. La frecuencia de muestreo fue de $500 \mathrm{~Hz}$ y se aplicó un filtro de paso alto (frecuencia de corte $=0.50 \mathrm{~Hz}$; constante de tiempo $=0.3 \mathrm{~s}$; pendiente $=12 \mathrm{~dB} /$ oct). Durante la grabación se utilizó como referencia al electrodo $\mathrm{Cz}$ y una vez finalizada, las señales fueron referenciadas de nuevo a la actividad promedio de todos los electrodos $(106,237)$.

Se realizaron registros EEG de 13 minutos de duración, empleándose un paradigma auditivo de rareza o del elemento extraño (paradigma oddball) para obtener el potencial evocado P300. Este paradigma de 3 estímulos incluía el estímulo diana (tono de $500 \mathrm{~Hz}$ ), el estímulo distractor (tono de $1000 \mathrm{~Hz}$ ) y el estímulo estándar (tono de $2000 \mathrm{~Hz}$ ) presentados con probabilidad de 0.20, 0.20 y 0.60 , respectivamente, en una serie aleatoria de 600 estímulos. Cada tono tenía una intensidad de $90 \mathrm{~dB}$, duración de $50 \mathrm{~ms}$ y tiempo de subida y bajada de $5 \mathrm{~ms}$, con un intervalo entre estímulos que osciló aleatoriamente entre 1.16 y $1.44 \mathrm{~s}$. Los participantes permanecieron sentados cómodamente en una habitación tranquila con los ojos cerrados y se les indicó que presionaran un botón del ratón cada vez que detectaran el estímulo diana. En los análisis solo se incluyeron los ensayos en los que el estímulo diana fue detectado de forma correcta por el participante. 


\subsubsection{Procesamiento del EEG y elaboración de las matrices de conectividad funcional}

Para eliminar el artefacto debido al movimiento de los ojos y a la contracción muscular se realizó un análisis de componentes independientes (ICA) para descartar los componentes ruidosos. Después de la reconstrucción ICA, las señales se dividieron en ensayos de $1 \mathrm{~s}$ (desde $300 \mathrm{~ms}$ antes del inicio del estímulo hasta $700 \mathrm{~ms}$ después). Los ensayos en los que la señal superaba un umbral adaptativo de amplitud se rechazaron de modo automático (238). Se aplicó un filtro de paso banda entre 1 y $70 \mathrm{~Hz}$, y un filtro de muesca de $50 \mathrm{~Hz}$ para eliminar el artefacto de la línea eléctrica.

Con base en estudios previos de la red funcional en esquizofrenia $(109,216)$, se aplicó un filtro de paso banda para obtener la señal EEG en la banda global $(1-70 \mathrm{~Hz})$ y en la banda $\theta(4-8 \mathrm{~Hz})$. Asimismo, en ambas bandas se delimitaron dos ventanas por cada ensayo: (i) la ventana preestímulo, formada por los $300 \mathrm{~ms}$ previos al inicio del tono diana, que corresponde a un periodo de expectativa antes del inicio del estímulo; y (ii) la ventana de respuesta, del $150 \mathrm{~ms}$ al $450 \mathrm{~ms}$ después del inicio del tono diana, que contiene el potencial P3b.

Para elaborar los grafos cerebrales basados en registros EEG, los nodos equivalieron a la representación matemática de los electrodos del EEG, mientras que los valores de las aristas de la red correspondió a una medida del acoplamiento neuronal entre cada par de electrodos (74). Este acoplamiento fue estimado, como se ha dicho previamente, con el valor de acoplamiento de fase (PLV) (239) a lo largo de ensayos sucesivos, un parámetro que es sensible a los componentes oscilatorios de baja amplitud del EEG (148) y a las no linealidades (240).

Para el cálculo del PLV se utilizó la transformada wavelet continua (CWT) para determinar el acoplamiento de fase en cada ensayo (241) considerando los conos de influencia para eliminar los efectos de borde (242). Aplicando el enfoque de la CWT para la realización del filtro y la extracción de fase en una sola operación, se obtuvo el PLV entre dos señales, $x(\mathrm{t})$ e $y(\mathrm{t})$, evaluando la variabilidad de la diferencia de fase a través de ensayos sucesivos. 
De este modo, se extrajo la fase instantánea de dos señales temporales, $x(\mathrm{t})$ e $y(\mathrm{t})$ y se calculó la variabilidad de la diferencia de fase de estas dos señales en los ensayos sucesivos, utilizando la siguiente fórmula $(109,239)$ :

$$
P L V_{x y}(k, s)=\frac{1}{N t}\left|\sum_{n=1}^{N} e^{\Delta \varphi_{x y}(k, s, n)}\right|,
$$

donde $N t$ es el número de ensayos, $\Delta \varphi_{x y}$ es la diferencia de fase instantánea entre las señales $x$ e $y, k$ es el intervalo de tiempo, y $s$ es el factor de escala de la wavelet madre.

Los pesos de las matrices de conectividad funcional (o matrices de adyacencia) se determinaron con el PLV entre cada par de electrodos. Debido al hecho de que no se aplicó ningún umbral, estas matrices de conectividad oscilaron entre 0 y 1 ; se obtuvo 0 cuando dos señales no tenían sincronización y 1 cuando dos señales se sincronizaron perfectamente, es decir que, a mayor sincronización entre los sensores, mayor PLV. Como las estimaciones de la conectividad cerebral se realizaron en dos bandas (banda $\theta /$ banda global) y dos ventanas (preestímulo/respuesta), por cada participante se construyeron 4 matrices de conectividad.

Para reducir los sesgos en un análisis de conectividad basado en señales registradas en el cuero cabelludo, se verificó que las conexiones con diferencias de fase de 0 o 180 ( \pm 1 grado) fueran menos del 1\%. De igual forma, para minimizar los efectos de conducción del volumen se siguió una estrategia fundamentada en la suposición de que la conducción del volumen afecta de manera similar las estimaciones de conectividad en dos contrastes experimentales diferentes, de modo que las estimaciones espurias se eliminan al comparar ambas condiciones (243).

\subsubsection{Procesamiento del EEG para el cálculo del ruido cortical}

Se aplicó un filtro paso banda de 0.5 a $70 \mathrm{~Hz}$ y un filtro de muesca de 50 $\mathrm{Hz}$ para eliminar el artefacto de línea eléctrica. Después, las grabaciones se dividieron en épocas de $650 \mathrm{~ms}$ con inicio a los $50 \mathrm{~ms}$ antes del estímulo diana. Estas épocas se corrigieron en la línea de base 
(equivalente al segmento de $50 \mathrm{~ms}$ antes del estímulo) y se rechazaron de modo automático cuando superaban un rango de $\pm 70 \mu \mathrm{V}$ en cualquiera de los 29 canales (244). A continuación, se realizó un ICA (software Brain Vision ${ }^{\circledR}$ ), para eliminar los artefactos de movimiento ocular y parpadeo. Además, se realizó una inspección visual para rechazar de manera manual las épocas restantes que aún presentaban cualquier otro artefacto. Los datos de los participantes se incluyeron en el análisis sólo si se disponía de al menos 40 épocas útiles.

\subsection{Cálculo de los parámetros para la valoración de la conectividad de la red y la actividad oscilatoria}

\subsubsection{Cálculo de los parámetros de la teoría de grafos}

A partir de las matrices de conectividad estructural y funcional se calculó la CS como el promedio la fuerza de todos los nodos de la red, que a su vez consiste en la suma de los pesos de todas sus aristas. Como se ha explicado, en el caso de las matrices de conectividad estructural el peso se estimó con la FA promedio de las streamlines que conformaron las aristas y en el caso de las matrices de conectividad funcional, con el PLV entre cada par de los nodos (109).

La CS se calculó con la siguiente fórmula:

$$
C S=\frac{\sum_{i=1}^{N} \sum_{j>i} w_{i j}}{T},
$$

donde $N$ denota el número de nodos del grafo, $w_{i j}$ denota el valor del peso de la arista entre $i$ y $j$, y $T=N(N-1) / 2$, que es el número total de conexiones de un grafo no dirigido (109).

En el estudio de la conectividad estructural se calcularon otros tres parámetros: el coeficiente de agrupamiento $C$, la longitud de camino característica $L$, y la relación entre éstos, denominado índice de mundo pequeño $(S W)$. El análisis se basó en el $S W$, de forma que en las comparaciones se encontraban diferencias significativas, éstas se caracterizaron con un análisis del $C$ y de la $L(100)$. 
El coeficiente de agrupamiento ponderado de la red $\left(C^{w}\right)$ se definió como:

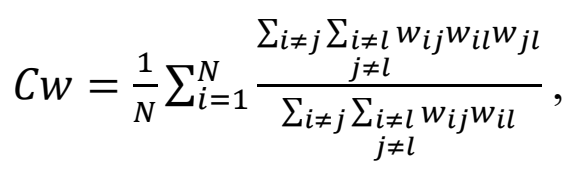

donde $N$ denota el número de nodos del grafo y $w_{i j}$ denota el valor del peso de la arista entre $i$ y $j(105,109)$.

Y la longitud de camino característica ponderada $\left(L_{w}\right)$ :

$$
L w=\frac{N(N-1)}{\sum_{i=1}^{N} \sum_{j \neq i}^{N} \frac{1}{L_{i j}}},
$$

donde $N$ denota el número de nodos del grafo y $L_{i j}$ denota la inversa del peso de la arista $w_{i j}(105,109)$.

Por definición $C_{\mathrm{w}}$ y $L_{\mathrm{w}}$ dependen no solo del peso de las aristas sino también del tamaño de la red. Para eliminar esta dependencia al tamaño de la red, en esta tesis los valores de $C_{\mathrm{w}}$ y $L_{\mathrm{w}}$ fueron normalizados así:

$$
C_{n}=\frac{C w}{C^{\text {random }}}
$$

y

$$
L_{n}=\frac{L w}{L^{\text {random }}},
$$

donde $C^{\text {random }}$ y $L^{\text {random }}$ denotan el $C$ y $L$ promediados sobre un conjunto de 50 redes construidas mediante el barajeo aleatorio de sus conexiones (105). De modo que el índice $S W$ se calculó como:

$$
S W=\frac{C n}{L n} .
$$

En el estudio de la conectividad funcional la CS fue calculada para cada ensayo en las ventanas preestímulo y respuesta, tanto en la banda global como en la banda $\theta$. Asimismo, en ambas bandas se calculó la modulación de la CS relacionada con la tarea cognitiva, definida como el cambio que experimentan los valores de CS entre las ventanas de preestímulo y respuesta (es decir, el valor de la CS en la ventana respuesta menos el valor de la CS en la ventana preestímulo). 


\subsubsection{Cálculo de potencia de ruido en la banda y (NP)}

Utilizando el programa informático de análisis del EEG (Brain Vision ${ }^{\circledR}$, Brain Products GmbH; Múnich, Alemania), de cada una de las épocas que contenían los estímulos diana se extrajo un segmento de $100 \mathrm{~ms}$ de duración con la respuesta P300, comprendido en el intervalo de 300 a $400 \mathrm{~ms}$ después del inicio del tono diana. Después, se aplicó un nuevo filtro de paso banda para obtener la señal en la banda $\gamma(35-45 \mathrm{~Hz})$.

En estos segmentos del registro, la NP de cada electrodo se calculó siguiendo un modelo lineal (173) a partir del valor de la potencia total de la señal (TP, total power) y de la relación señal/ruido (SNR, signal to noise ratio) $(171,172,224)$. La TP promedio, que es la amplitud cuadrática media expresada en $\mu \mathrm{V}^{2}$ de todos los ensayos promediados (174) se estimó con un análisis espectral usando la transformada rápida de Fourier (FFT). El valor de la SNR fue proporcionado por el programa de análisis Brain Analyzer (172,224).

Debido a que:

$$
T P=S P+N P,
$$

donde $T P$ es la potencia total, $S P$ la potencia de la señal y $N P$ la potencia de ruido.

Y a que:

$$
S N R=\frac{S P}{N P},
$$

la $N P$ se obtiene a partir del valor del $S N R$ y del $T P$ usando la siguiente fórmula:

$$
N P=\frac{T P}{S N R+1} .
$$

\subsection{Análisis estadístico}

Dados los tamaños de muestra de los grupos y la distribución no normal de muchos datos, para el análisis estadístico se eligieron test no paramétricos. El nivel de significación estadística del contraste de hipótesis fue establecido con un valor $p<0.05$. 
En los tres estudios realizados, de las anormalidades encontradas en los parámetros analizados se determinó: (a) la especificidad para el diagnóstico, (b) la relación con la duración de la enfermedad, (c) la relación con el tratamiento farmacológico y (d) la relación con los síntomas y con la cognición.

\subsubsection{Artículo 1: Structural connectivity in schizophrenia and bipolar disorder: Effects of chronicity and antipsychotic treatment}

Se realizaron comparaciones entre los cuatro grupos de participantes (EPE, EC, TB y CTRL) de la distribución de sexo (prueba de la $\chi^{2}$ ) y de la distribución de edad y de las puntuaciones de las escalas cognitivas utilizando un análisis de varianza (prueba de Kruskal-Wallis, post hoc $U$ de Mann-Whitney). Asimismo, se realizaron comparaciones entre los tres grupos de pacientes de las puntuaciones de las escalas de síntomas, de las dosis de tratamiento antipsicótico y de la duración de la enfermedad (prueba de Kruskal-Wallis, post hoc $U$ de Mann-Whitney).

(a) Se compararon los parámetros de red (CS y $\mathrm{SW}$ ) entre los grupos EC, TB y CTRL (prueba de Kruskall-Wallis). Al detectar un efecto de grupo significativo, se realizaron comparaciones post hoc para identificar los grupos que diferían (prueba $U$ de Mann-Whitney). Dado que el SW es la relación entre $\mathrm{C}$ y L, en los grupos con diferencias significativas del $\mathrm{SW}$ se analizaron estos otros valores.

(b) Se compararon los parámetros de red entre los grupos EPE y EC (prueba $U$ de Mann-Whitney). Además, se realizó el cálculo de los coeficientes de correlación de Spearman $(\rho)$ entre los parámetros de red y la duración de la enfermedad.

(c) Se compararon los parámetros de red entre pacientes con TB tratados con antipsicóticos $(\mathrm{n}=13)$ y sin este tratamiento durante al menos los últimos seis meses $(\mathrm{n}=11)$ (prueba $U$ de Mann-Whitney). Asimismo, se realizó el cálculo de los coeficientes de correlación de Spearman $(\rho)$ entre los parámetros de red y la dosis actual de antipsicóticos (en equivalentes de clorpromazina). 
(d) Se realizó el cálculo de los coeficientes de correlación de Spearman $(\rho)$ entre los parámetros de red y (i) las puntuaciones del rendimiento cognitivo (BACS, errores perseverativos del WCST y cognición social) y (ii) las puntuaciones de las escalas de síntomas (PANSS positivo y total y BNSS total).

\subsubsection{Artículo 2: Connectivity strength of the EEG functional network in schizophrenia and bipolar disorder}

Se compararon las distribuciones por edad y sexo entre los grupos ESQ, TB y CTRL (análisis de varianza -ANOVA - y prueba de la $\chi^{2}$, respectivamente). Se compararon las dosis de tratamiento y la duración de la enfermedad entre los pacientes (prueba $t$ de Student para muestras independientes). Se determinó la normalidad de la distribución de los valores de CS en cada uno de los grupos (prueba de KolmogorovSmirnov).

(a) Se compararon los valores de la CS preestímulo en la banda global y en la banda $\theta$ y la modulación de CS en la banda global y en la banda $\theta$ entre los grupos (análisis multivariado de la covarianza MANCOVA-, con los valores de CS como variables dependientes y la edad como covariable). Al encontrar un efecto significativo del grupo, se realizaron comparaciones de grupos por pares para identificar los grupos que diferían. Los tamaños del efecto se calcularon usando los valores de $\eta^{2}$.

(b) Se compararon los valores alterados de la CS entre los grupos EC y EPE, (prueba $t$ de Student para muestras independientes).

(c) Se calcularon las correlaciones $r$ de Pearson entre los valores alterados de la CS y la dosis actual de antipsicóticos (en equivalentes de clorpromazina). Los valores alterados de la CS se compararon (i) entre los pacientes TB que recibieron y no recibieron antipsicóticos y (ii) entre los pacientes que recibieron y no recibieron los demás tipos de psicofármacos: litio —solo pacientes TB-, benzodiacepinas, anticonvulsivos o antidepresivos (prueba $U$ de Mann-Whitney).

(d) Se realizó un análisis de componentes principales para obtener un factor de cognición general a partir de las puntuaciones cognitivas 
individuales de todos los participantes. Se utilizó un análisis de regresión lineal múltiple para evaluar las relaciones entre los valores de la CS y (i) las puntuaciones del factor de cognición general y (ii) las puntuaciones de las escalas de síntomas.

\subsubsection{Artículo 3: Altered gamma band noise power in schizophrenia and bipolar patients during a cognitive task}

Entre los tres grupos ESQ, TB y CTRL se comparó (i) la distribución de sexo (prueba de la $\chi^{2}$ ) y (ii) la distribución de edad, los años de educación, las puntuaciones de BACS, la amplitud de P300 y las variables de rendimiento durante la tarea oddball (ANOVA, Bonferroni post hoc). Entre los grupos ESQ y TB se compararon las puntuaciones de la PANSS, las dosis de tratamiento y la duración de la enfermedad (prueba $t$ de Student para muestras independientes). Se determinó la normalidad de los valores de la NP- $\gamma$ (pruebas de KolmogorovSmirnov).

(a) Se compararon los valores de la NP- $\gamma$ entre los grupos (prueba de permutación basada en estadísticas $t$ utilizando FieldTrip para MATLAB). Se calculó una estimación de Montecarlo de las probabilidades de significancia o valores críticos, basado en aleatorizar los datos 1000 veces entre las condiciones del estudio. El error de tipo I en las comparaciones múltiples se controló mediante la corrección de Bonferroni $(p=0.05 / 29=0.002)$.

(b) Se evaluó la relación entre los valores alterados de la NP- $\gamma$ y la duración de la enfermedad (coeficiente de correlación $\rho$ de Spearman). Asimismo, esta relación se evaluó mediante un análisis predictivo con un modelo de regresión múltiple tomando cada valor alterado de la NP$\gamma$ como variable dependiente y la duración de la enfermedad como variable independiente.

(c) Se evaluó la relación entre los valores alterados de la NP- $\gamma$ y la dosis de antipsicóticos, en equivalentes de clorpromazina (coeficiente de correlación $\rho$ de Spearman). Asimismo, esta relación se evaluó mediante un análisis predictivo con un modelo de regresión múltiple tomando cada valor alterado de la NP- $\gamma$ como variable dependiente y la dosis actual de 
antipsicóticos como variable independiente. Además, los valores alterados de la NP- $\gamma$ se compararon (i) entre los pacientes TB que recibieron y no recibieron antipsicóticos y (ii) entre los pacientes que recibieron y no recibieron los demás tipos de psicofármacos: litio — solo pacientes TB-, benzodiacepinas, anticonvulsivos o antidepresivos (test de permutación con simulación de Montecarlo - FieldTrip).

(d) Se realizó un análisis de componentes principales para obtener los factores de cognición general a partir de las puntuaciones cognitivas individuales de todos los participantes. Se realizó el cálculo de los coeficientes de correlación de Spearman $(\rho)$ entre los valores alterados de la NP- $\gamma$ y las puntuaciones de síntomas (PANSS) y cognitivas (factores de cognición general y las variables de rendimiento de la tarea oddball). En el grupo ESQ se realizó un análisis de regresión múltiple para evaluar las relaciones entre cada valor alterado de NP- $\gamma$ (variable dependiente) y las puntuaciones del rendimiento cognitivo y de los síntomas (variables independientes). 


\section{CAPÍTULO 4}

\section{Resultados}

os resultados de cada uno de los artículos de investigación se detallan en las respectivas publicaciones incorporadas en los anexos de esta memoria. En esta sección se expone un extracto de cada uno de ellos traducido a la lengua española y una adaptación de las tablas y figuras que resumen los resultados más relevantes.

\subsection{Conectividad estructural de la red cerebral} Artículo 1: Structural connectivity in schizophrenia and bipolar disorder: Effects of chronicity and antipsychotic treatment.

\subsubsection{Resultados demográficos, clínicos y cognitivos}

- Distribución por sexo. No hubo diferencias significativas entre los grupos $\left(\chi^{2}=1.428, \mathrm{gl}=3, p=0.699\right)$ (Tabla 2).

- Distribución por edad. Hubo diferencias significativas entre los grupos $\left(\chi^{2}=27.13, \mathrm{gl}=3, p=<0.001\right)$ : el grupo TB era mayor que el $\operatorname{EPE}(U=34.5, \mathrm{z}=4.53, p<0.001)$ y CTRL $(U=139, \mathrm{z}=3,47, p$ $<0.001)$. Igualmente, la edad del grupo EC era mayor que la del grupo EPE $(U=84.5, \mathrm{z}=3.22, p<0.001)$. Sin embargo, no hubo diferencias significativas al comparar los grupos EPE y EC con CTRL. 
- Escalas de valoración cognitiva. Hubo diferencias significativas entre los grupos: los pacientes mostraron puntuaciones más bajas en comparación con los CTRL en el coeficiente intelectual total y en todos los dominios de la BACS, excepto en la torre de Londres.

- Duración de la enfermedad. Hubo diferencias significativas entre los grupos: la del grupo EC fue mayor en comparación con EPE ( $U$ $=14.5, \mathrm{z}=4.66, p<0.001)$ y la del grupo TB fue mayor en comparación con $\operatorname{EPE}(U=14, \mathrm{z}=4.68, p<0.001)$. No hubo diferencias significativas entre los grupos de EC y TB $(U=204, \mathrm{z}=$ $0.891, p<0.372)$.

\subsubsection{Especificidad para el diagnóstico de las alteraciones de los parámetros de conectividad estructural}

- CS y SW. Se encontró un efecto de grupo significativo para la $C S\left(\chi^{2}\right.$ $=16.391, \mathrm{gl}=2, p<0.001)$ y para el $S W\left(\chi^{2}=6.096, \mathrm{gl}=2, p=\right.$ 0.042) (Fig. 8 y Tabla 3).

- CS. En el grupo EC fue menor en comparación con los CTRL (U = $161, \mathrm{z}=-3.367, p=0.001)$ pero en comparación con TB no hubo diferencias significativas $(U=279, \mathrm{z}=-0.420, p=0.368)$.

- $\mathbf{S W}$. En el grupo EC fue menor en comparación con los CTRL ( $U=$ 212, $\mathrm{z}=-2,459, p=0.014)$ pero en comparación con TB no hubo diferencias significativas $(U=255, \mathrm{z}=-0.900, p=0.368)$.

C y L. Al comparar los grupos EC y CTRL no hubo diferencias significativas en el $C_{\mathrm{n}}$, pero hubo una diferencia significativa en el $L_{\mathrm{n}}$, que fue mayor en el grupo $\mathrm{EC}(U=227, \mathrm{z}=2.192, p=0.014)$. 
Tabla 2 | Resultados demográficos, cognitivos, clínicos y del tratamiento en el estudio de conectividad estructural

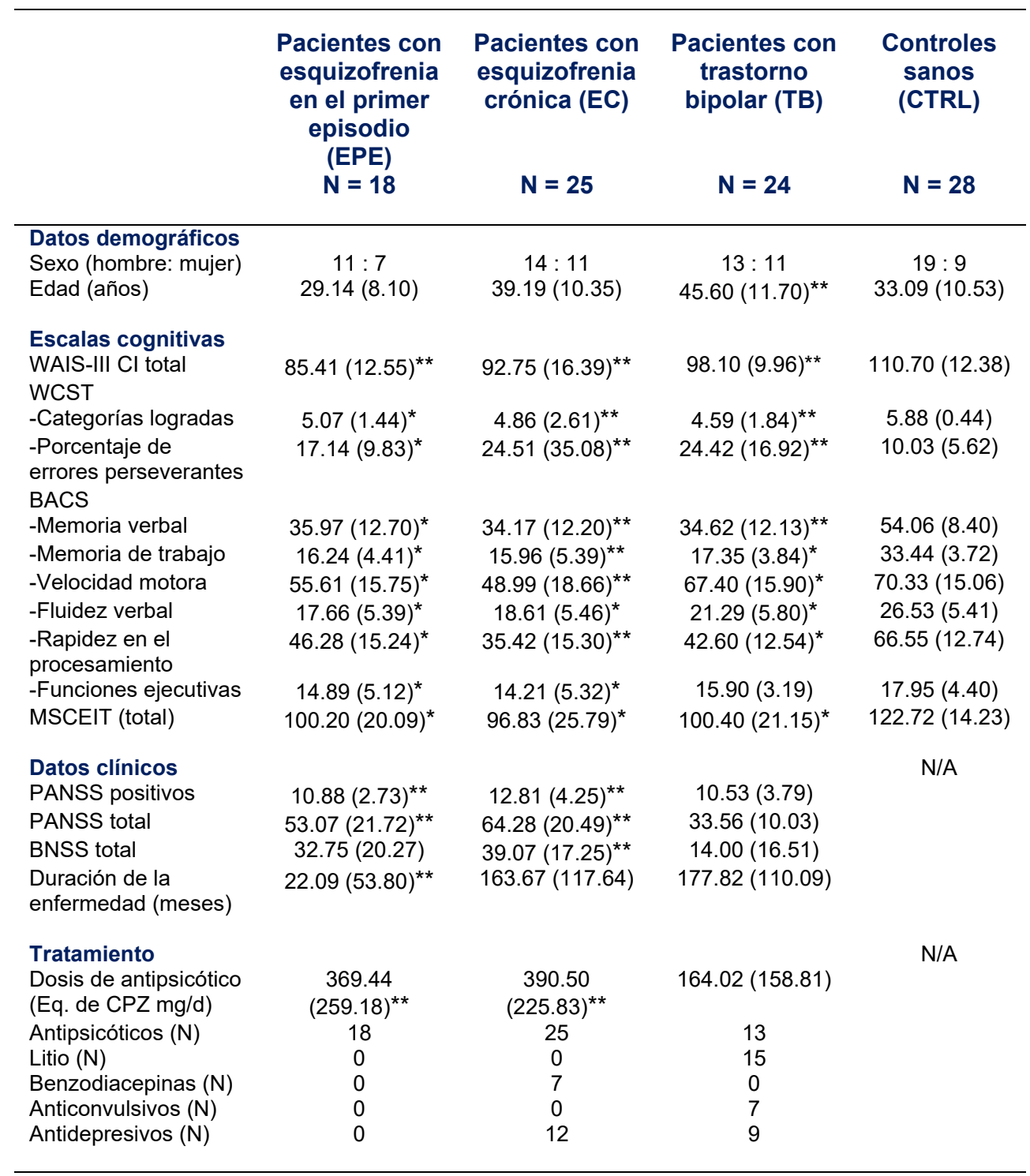

Para la edad, las escalas cognitivas y los datos clínicos se muestran los valores de la media y desviación estándar (SD). Se representa la significación estadística de la comparación entre los pacientes y controles (edad y escalas cognitivas) y de la comparación entre pacientes con esquizofrenia y con trastorno bipolar (datos clínicos y dosis de antipsicótico). ${ }^{*} p<0.05 ;{ }^{* *} p<0.01$. WAIS-III, Escala de inteligencia del adulto de Wechsler; $\mathrm{Cl}$, cociente intelectual; WCST, Test de clasificación de tarjetas de Wisconsin; BACS, Escala de evaluación breve de la cognición en la esquizofrenia; MSCEIT, Prueba de inteligencia emocional Mayer-Salovey-Caruso; PANSS, Escala para el síndrome positivo y negativo de la esquizofrenia; BNSS, Escala breve de síntomas negativos; Eq. de CPZ, equivalentes de clorpromazina; N/A, no es aplicable. 
A

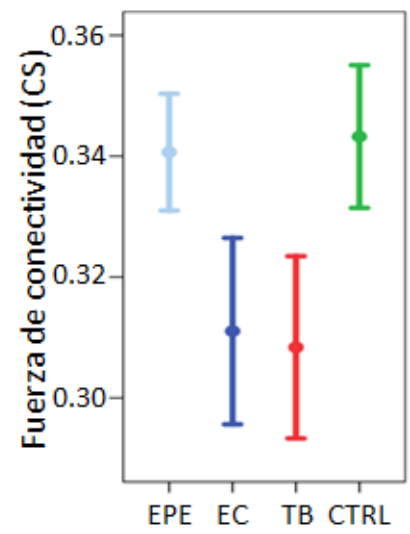

B

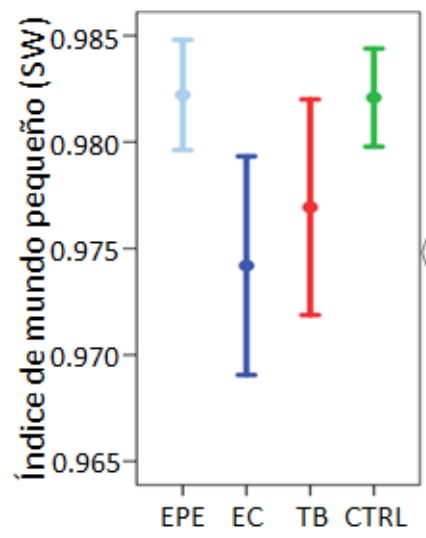

C

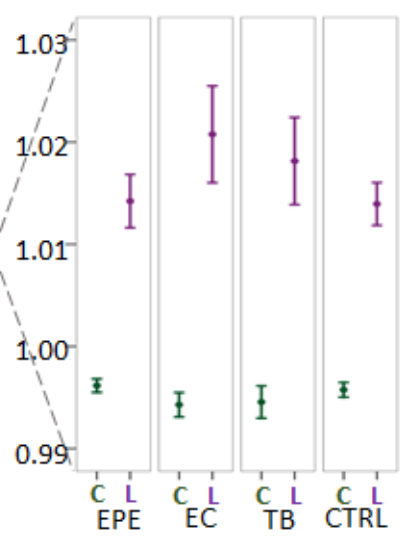

Fig. 8| Fuerza de conectividad e índice de mundo pequeño de la red estructural. (A-B) Intervalos de confianza del 95\% de la fuerza de conectividad (CS) e índice de mundo pequeño (SW). (C) EI SW indica la relación entre el coeficiente de agrupamiento (C) y la longitud de camino característica $(\mathrm{L})$ de la red. Los pacientes con EC y TB mostraron valores de SW más pequeños y $L$ más grandes que los $C T R L$, pero no hubo diferencias significativas en el $C$ entre los grupos. EPE, pacientes con esquizofrenia en el primer episodio; EC, pacientes con esquizofrenia crónica; TB, pacientes con trastorno bipolar; CTRL, controles sanos.

Tabla 3 | Valores de fuerza de conectividad (CS) e índice de mundo pequeño (SW)

\begin{tabular}{|c|c|c|c|c|}
\hline $\begin{array}{c}\text { Parámetros } \\
\text { de } \\
\text { conectividad }\end{array}$ & $\begin{array}{l}\text { Pacientes con } \\
\text { esquizofrenia } \\
\text { en el primer } \\
\text { episodio (EPE) } \\
\mathrm{N}=18\end{array}$ & $\begin{array}{l}\text { Pacientes con } \\
\text { esquizofrenia } \\
\text { crónica } \\
\text { (EC) } \\
\text { N = 25 }\end{array}$ & $\begin{array}{c}\text { Pacientes con } \\
\text { trastorno } \\
\text { bipolar } \\
\text { (TB) } \\
\text { N = 24 }\end{array}$ & $\begin{array}{c}\text { Controles } \\
\text { sanos } \\
\text { (CTRL) } \\
\text { N = 28 }\end{array}$ \\
\hline CS & $0.341(0.019)$ & $0.311(0.037)^{\star *}$ & $0.304(0.035)^{* *}$ & $0.343(0.030)$ \\
\hline SW & $0.982(0.005)$ & $0.974(0.012)^{*}$ & $0.976(0.012)^{*}$ & $0.982(0.006)$ \\
\hline$-C$ & $0.996(0.001)$ & $0.994(0.003)$ & $0.994(0.004)$ & $0.996(0.002)$ \\
\hline$-L$ & $1.014(0.005)$ & $1.021(0.011)^{* *}$ & $1.018(0.010)^{*}$ & $1.014(0.005)$ \\
\hline
\end{tabular}

El índice de mundo pequeño indica la relación entre el coeficiente de agrupamiento $(\mathrm{C})$ y la longitud de camino característica (L) de la red. Se muestran los valores de la media y desviación estándar (SD). Se representa la significación estadística de la comparación entre los pacientes y controles. ${ }^{*} p<0.05,{ }^{* *} p<0.001$. 


\subsubsection{Relación de las alteraciones de los parámetros de conectividad estructural con la duración de la enfermedad}

- CS. Hubo una diferencia significativa al comparar los grupos EC y $\operatorname{EPE}(U=103, \mathrm{z}=-3.003, p=0.003)$, siendo menor en $\mathrm{EC}$.

No hubo diferencias significativas entre EPE y CTRL $(U=231, \mathrm{z}=$ 0.473, $p=0.636$ ) (Fig. 9).

- SW. Hubo una diferencia significativa al comparar el grupo EC y EPE $(U=128, \mathrm{z}=-2.388, p=0.017)$ siendo menor en EC. Igualmente, entre estos grupos hubo diferencias significativas en el $C$ y $L$ : en el grupo EC el $C_{\mathrm{n}}$ fue menor $(U=135, \mathrm{z}=-2.21, p=0.027)$ y el $L_{\mathrm{n}}$ fue mayor $(U=143, \mathrm{z}=2.043, p=0.04)$.

No hubo diferencias significativas del $S W$ entre el grupo EPE y CTRL.

- Correlación entre CS y SW y la duración de la enfermedad. Al considerar en conjunto a los tres grupos de pacientes, hubo una correlación inversa significativa entre la duración de la enfermedad y los valores de la $C S(\rho=-0.336, p=0.004)$ y del $S W(\rho=-0.374$, $p=0.004)$.

Al considerar solo los grupos de pacientes con esquizofrenia (EPE y $\mathrm{EC})$, aún hubo una correlación significativa para la $C S(\rho=-0.422$, $p=0.009)$ y el $S W(\rho=-0.515, p=0.001)$. Pero al considerar solo el grupo TB la correlación ya no fue significativa para la $C S$ ( $\rho=$ $-0.007, p=0.976)$ ni para el $S W(\rho=-0.179, p=0.427)$.

\subsubsection{Relación de las alteraciones de los parámetros de conectividad estructural con el tratamiento farmacológico}

- CS. No hubo diferencia significativa entre los pacientes TB tratados $(0.259, \mathrm{SD} 0.033)$ y no tratados $(0.320, \mathrm{SD} 0.037)$ con antipsicóticos $(U=46, \mathrm{z}=-1.178, p=0.257)$. 

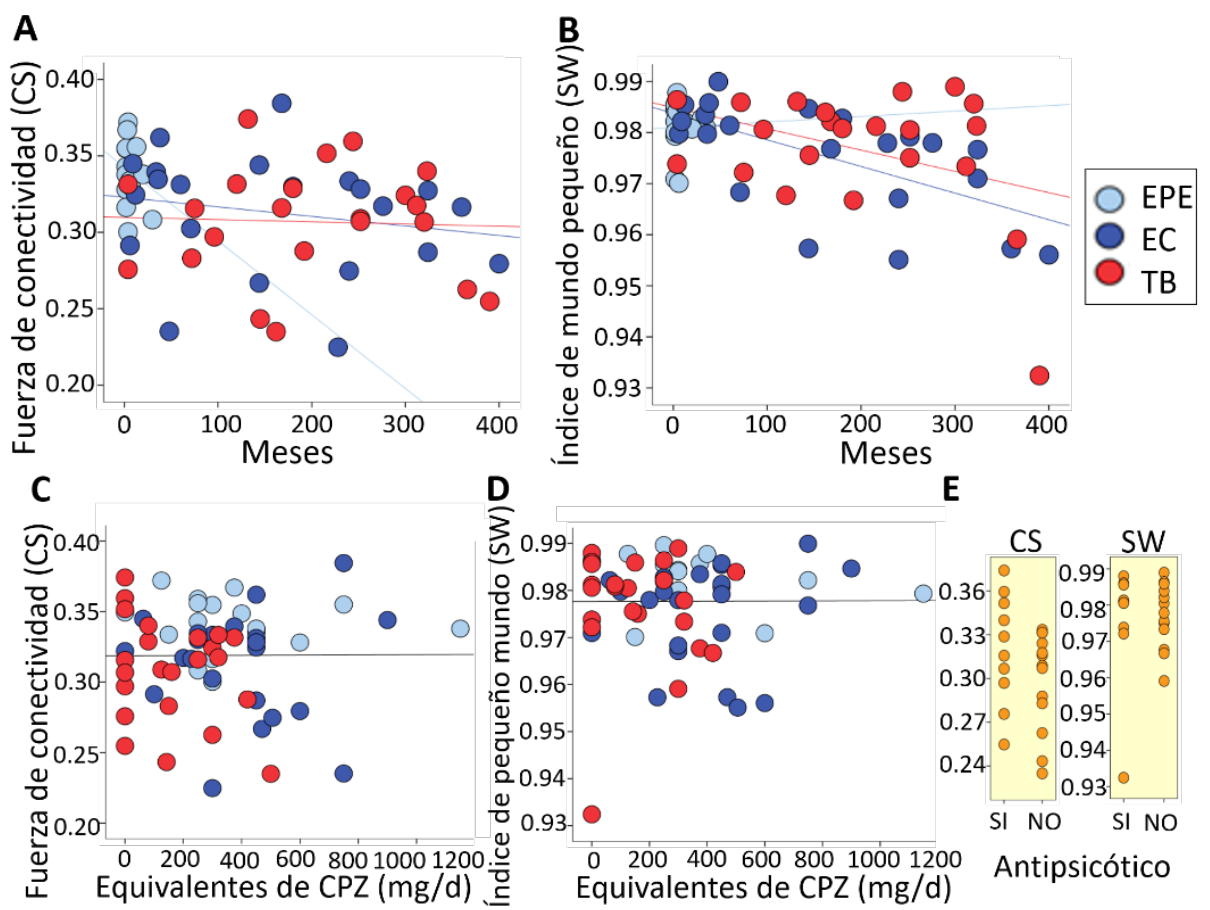

Fig. 9 Relación de los valores de fuerza de conectividad e índice de mundo pequeño con la duración de la enfermedad y el tratamiento antipsicótico. (A-B) Diagramas de dispersión que muestran la correlación entre los valores de la fuerza de conectividad (CS) y del índice de mundo pequeño (SW) y la duración de la enfermedad. La correlación fue significativa en la esquizofrenia en ambos casos, pero no en el grupo bipolar. (C-D) Diagramas de dispersión que muestran la ausencia de correlación entre la dosis de antipsicótico y los valores de CS y SW. (E) Comparación de los valores de CS y SW entre pacientes bipolares con o sin tratamiento antipsicótico. EPE, pacientes con esquizofrenia en el primer episodio; EC, pacientes con esquizofrenia crónica; TB pacientes con trastorno bipolar.

- SW. No hubo diferencias significativas entre los pacientes TB tratados $(0.977$, SD 0.008$)$ y no tratados $(0.975$, SD 0.018) con antipsicóticos $(U=57, \mathrm{z}=-0.194, p=0.875)$.

Las comparaciones del $C_{\mathrm{n}}$ entre pacientes TB tratados $(0.994, \mathrm{SD}$ $0.003)$ y no tratados con antipsicóticos $\left(0.994\right.$, SD 0.005) y la del $L_{\mathrm{n}}$ entre pacientes con TB tratados (1.017, SD 0.007) y no tratados con antipsicóticos (1.019, SD 0.014) tampoco mostraron diferencias significativas. 
No hubo diferencias significativas entre la duración de la enfermedad de los pacientes TB tratados (210.9, SD 96.60 meses) y no tratados (65.29, SD 128.67) con antipsicóticos $(U=35, \mathrm{z}=-1.04, p=.32)$.

- Correlación entre CS y SW y la dosis de antipsicótico. No hubo correlación significativa entre la dosis actual de antipsicótico y la $C S$ ni el $S W$, tanto para todos los pacientes tomados en conjunto $(-0.059$ $<\rho<0.060)$ como para los pacientes con esquizofrenia y $\mathrm{TB}$ considerados por separado $(0.103<\rho<0.114)$.

\subsubsection{Relación de las alteraciones de los parámetros de conectividad estructural con la cognición y los síntomas}

En todos los pacientes considerados en conjunto:

- CS. Tuvo una correlación directa con la puntuación en la memoria de trabajo $(\rho=0.291, p=0.039)$.

- SW. Tuvo una correlación directa con la puntuación en las funciones ejecutivas — torre de Londres—; $(\rho=0.289, p=0.040)$ y correlación inversa con el porcentaje de errores perseverativos del WCST $(\rho=$ $-0.321, p=0.025$ ) (Fig. 10).

En los pacientes con esquizofrenia (EPE y EC):

- CS. No tuvo correlación significativa con los síntomas ni con las puntuaciones cognitivas.

- SW. Tuvo una correlación directa con la puntuación en las funciones ejecutivas - torre de Londres- $(\rho=0.332, p=0.048)$ y con la puntuación total en la cognición social -MSCEIT- $(\rho=0.372, p=$ 0.047). Asimismo, tuvo una correlación inversa con el porcentaje de errores perseverativos del WCST ( $\rho=-0.358, p=0.035)$.

En los pacientes con trastorno bipolar:

- CS. Tuvo una correlación directa con las puntuaciones de la cognición social $(\rho=0.547, p=0.028)$.

- SW. Tuvo una correlación inversa con los síntomas positivos $(\rho=$ $-0.661, p=0.001$ ).

- No hubo correlaciones significativas entre la CS y el SW y las puntuaciones de la cognición general. 
A

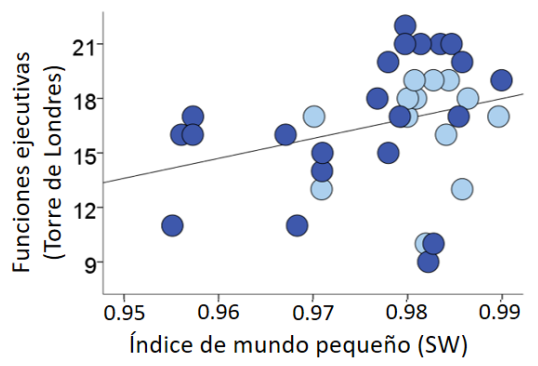

B

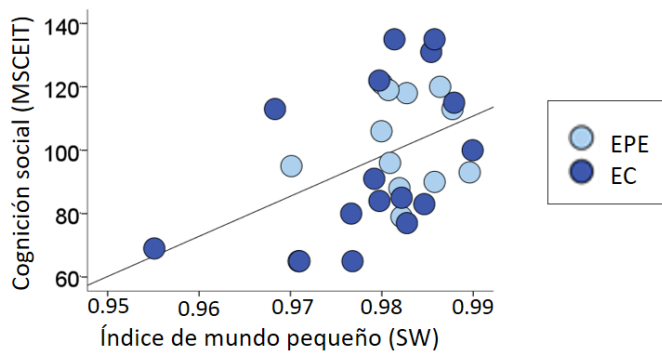

Fig. 10| Relación de los valores del índice de mundo pequeño con las puntuaciones cognitivas en la esquizofrenia. Diagramas de dispersión que muestran la correlación directa entre los valores del índice de mundo pequeño (SW) y (A) las puntuaciones del dominio de funciones ejecutivas y (B) las puntuaciones de la cognición social. MSCEIT, Prueba de inteligencia emocional de Mayer-Salovey-Caruso. EPE, pacientes con esquizofrenia en el primer episodio; EC, pacientes con esquizofrenia crónica.

\subsection{Conectividad funcional de la red cerebral}

Artículo 2: Connectivity strength of the EEG functional network in schizophrenia and bipolar disorder.

\subsubsection{Resultados demográficos, clínicos y cognitivos}

- Distribución por sexo. No hubo diferencias significativas entre los grupos $\left(\chi^{2}=2.05, p=0.358\right)$ (Tabla 4$)$.

- Distribución por edad. Hubo diferencias significativas entre los grupos: el grupo TB era mayor en comparación con ESQ y CTRL ( $\mathrm{F}$ $=10.66, p<0.001)$.

- Duración de la enfermedad. Hubo una diferencia significativa entre los grupos: el grupo TB tenía una mayor duración de la enfermedad comparado con $\operatorname{ESQ}(t=-2.95, p=0.004)$.

- Tratamiento farmacológico. Hubo una diferencia significativa entre los grupos: el grupo ESQ recibía dosis más altas de antipsicóticos comparado con TB $(t=3.35, p=0.001)$. 
Tabla 4. Resultados demográficos, cognitivos, clínicos y del tratamiento en el estudio de conectividad funcional

\begin{tabular}{|c|c|c|c|}
\hline & $\begin{array}{c}\text { Pacientes con } \\
\text { esquizofrenia } \\
\text { (ESQ) } \\
N=79\end{array}$ & $\begin{array}{l}\text { Pacientes con } \\
\text { trastorno bipolar } \\
\text { (TB) } \\
N=29\end{array}$ & $\begin{array}{l}\text { Controles } \\
\text { sanos } \\
\text { (CTRL) } \\
N=63\end{array}$ \\
\hline \multicolumn{4}{|l|}{ Datos demográficos } \\
\hline $\begin{array}{l}\text { Sexo } \\
\text { (hombre: mujer) }\end{array}$ & $48: 31$ & $17: 12$ & $32: 31$ \\
\hline Edad (años) & $35.66(10.57)$ & $46.41(10.45)^{\star *}$ & $38.19(10.14)$ \\
\hline \multicolumn{4}{|l|}{ Escalas cognitivas } \\
\hline WAIS-III CI total & $92.06(14.73)^{\star *}$ & $98.32(10.23)^{\star *}$ & $114.02(11.44)$ \\
\hline $\begin{array}{l}\text { BACS } \\
\text {-Memoria verbal }\end{array}$ & $34.72(13.44)^{\star *}$ & $36.00(9.25)^{\star \star}$ & $49.12(8.37)$ \\
\hline -Memoria de trabajo & $16.41(4.73)^{* *}$ & $17.58(4.03)^{* *}$ & $21.44(3.37)$ \\
\hline -Velocidad motora & $59.05(19.31)^{\star *}$ & $66.11(13.67)^{*}$ & 73.91 (16.64) \\
\hline -Fluidez verbal & $17.13(7.10)^{* *}$ & $19.79(7.87)^{\star *}$ & $24.78(9.88)$ \\
\hline $\begin{array}{l}\text {-Rapidez en el } \\
\text { procesamiento }\end{array}$ & $41.94(14.59)^{* *}$ & $41.48(12.40)^{\star *}$ & $64.93(13.74)$ \\
\hline -Funciones ejecutivas & $16.02(4.36)$ & $15.95(3.30)$ & $17.11(3.25)$ \\
\hline $\begin{array}{l}\text { Factor cognitivo } \\
\text { general }\end{array}$ & $-0.84(0.89)^{\star *}$ & $-0.79(0.69)^{\star *}$ & $0.31(0.62)$ \\
\hline \multicolumn{3}{|l|}{ Datos clínicos } & N/A \\
\hline PANSS-P positiva & $11.07(3.62)$ & $7.37(0.83)$ & \\
\hline PANSS-N negativa & $16.50(7.26)$ & $9.05(2.48)$ & \\
\hline YMRS & $0.83(1.91)$ & $1.81(1.34)$ & \\
\hline HDRS & $5.87(5.09)$ & $4.98(3.81)$ & \\
\hline $\begin{array}{l}\text { Duración de la } \\
\text { enfermedad (meses) }\end{array}$ & $101.69(116.10)$ & $182.22(106.04)^{* *}$ & \\
\hline \multicolumn{3}{|l|}{ Tratamiento (N) } & N/A \\
\hline $\begin{array}{l}\text { Dosis de antipsicótico } \\
\text { (Eq de CPZ mg/d) }\end{array}$ & $411.21(377.28)^{* *}$ & $157.40(152.90)$ & \\
\hline Antipsicóticos & 48 & 15 & \\
\hline Litio & 0 & 18 & \\
\hline Benzodiacepinas & 27 & 7 & \\
\hline Anticonvulsivos & 0 & 4 & \\
\hline Antidepresivos & 15 & 6 & \\
\hline \multicolumn{4}{|l|}{ Neurofisiología } \\
\hline P3b amplitud $(\mu \mathrm{V})$ & $1.04(0.61)$ & $1.23(0.63)$ & $1.58(0.76)$ \\
\hline P3b latencia (ms) & $423.22(95.43)$ & $464.69(96.11)$ & $458.54(71.40)$ \\
\hline
\end{tabular}

Para la edad, las escalas cognitivas, los datos clínicos y de neurofisiología se muestran los valores de la media y desviación estándar (SD). Se representa la significación estadística de la comparación entre los pacientes y CTRL o la comparación entre los pacientes. ${ }^{*} p<0.05 ;{ }^{* *} p<0.01$. WAIS-III, Escala de inteligencia del adulto de Wechsler; $\mathrm{Cl}$, cociente intelectual; BACS, Escala de evaluación breve de la cognición en la esquizofrenia; PANSS, Escala para el síndrome positivo y negativo de la esquizofrenia YMRS, Escala de manía de Young; HDRS, Escala de evaluación para la depresión de Hamilton; Eq. de CPZ, equivalentes de clorpromazina; N/A, no es aplicable. 
Tabla 5 Valores de fuerza de conectividad (CS) en la ventana preestímulo y su modulación durante la tarea cognitiva en las bandas global (BG) y $\theta$

\begin{tabular}{|c|c|c|c|}
\hline $\begin{array}{l}\text { Parámetros de } \\
\text { conectividad }\end{array}$ & $\begin{array}{c}\text { Pacientes con } \\
\text { esquizofrenia } \\
\text { (ESQ) } \\
N=79\end{array}$ & $\begin{array}{c}\text { Pacientes con } \\
\text { trastorno } \\
\text { bipolar (TB) } \\
\mathrm{N}=29\end{array}$ & $\begin{array}{c}\text { Controles } \\
\text { sanos (CTRL) } \\
\text { N = 63 }\end{array}$ \\
\hline CS preestímulo BG & $0.3136(0.0438)^{*}$ & $0.2963(0.0337)$ & $0.2981(0.0425)$ \\
\hline CS preestímulo $\theta$ & $0.3600(0.0448)$ & $0.3486(0.0397)$ & $0.3507(0.0364)$ \\
\hline Modulación CS BG & $-0.0003(0.0084)$ & $-0.0030(0.0081)$ & $0.0005(0.0124)$ \\
\hline Modulación CS $\theta$ & $0.0195(0.0298)^{* * *}$ & $0.0192(0.0236)^{* * *}$ & $0.0636(0.0467)$ \\
\hline
\end{tabular}

La modulación de la CS indica la diferencia entre las ventanas preestímulo y respuesta durante la tarea cognitiva. Se muestran los valores de la media y desviación estándar (SD). Se representa la significación estadística de la comparación entre los pacientes con esquizofrenia y trastorno bipolar $\left({ }^{*} p<0.05\right)$ y entre los pacientes y controles $\left({ }^{* *} p<0.001\right)$.

\subsubsection{Especificidad para el diagnóstico de las alteraciones en los parámetros de conectividad funcional}

- CS preestímulo y modulación de la CS en la banda global y $\boldsymbol{\theta}$. Los valores de CS preestímulo y de su modulación durante una tarea cognitiva en las bandas global y $\theta$ tuvieron una distribución normal en cada grupo (Tabla 5).

- El análisis multivariado mostró un efecto de grupo significativo $(\lambda$ de Wilks $\left.=7.44, \mathrm{gl}=8.318, p<0.001 ; \eta^{2}=0.158\right)$.

Las pruebas de los efectos inter-sujetos revelaron un efecto significativo de grupo para la CS preestímulo en la banda global ( suma de cuadrados tipo $\mathrm{III}=0.012, \mathrm{~F}=3.30, \mathrm{gl}=2, p=0.04 ; \eta^{2}=$ 0.04 ) y para la modulación de la CS en la banda $\theta$ (suma de cuadrados tipo III $=0.037, \mathrm{~F}=27.48, \mathrm{gl}=2, p<0.0001 ; \eta^{2}=0.25 ;$ ). No hubo diferencias significativas entre los grupos en la CS preestímulo $\theta$.

- CS preestímulo en la banda global. Fue más alto en ESQ en comparación con CTRL (diferencia de medias $=0.016$; $\mathrm{IC}_{95 \%}=$ $0.002-0.031, p=0.027$ ) y TB (diferencia de medias $=0.020 ;$ IC95\% 
$=0.000-0.040, p=0.049)$. No hubo diferencias significativas al comparar TB con CTRL (diferencia de medias $=-0.004$; $\mathrm{IC}_{95} \%=$ $-0.024-0.016, p=0.70)$ (Fig. 11).

- Modulación de la CS en la banda $\boldsymbol{\theta}$. Fue significativamente menor en ESQ en comparación con CTRL (diferencia de medias $=-0.043$; $\left.\mathrm{IC}_{95 \%}=-0.055 \mathrm{a}-0.031, p<0.001\right)$ y TB (diferencia de medias $=$ -0.044 ; IC95\% $=-0.061$ a $-0.027, p<0.001$ ) (Fig. 12).

- Análisis regional de los valores de CS preestímulo en la banda global y la modulación de la CS en la banda $\boldsymbol{\theta}$. Las diferencias significativas observadas al comparar CTRL y ESQ fueron mayores que al comparar CTRL y TB. De la CS preestímulo en la banda global las principales diferencias se ubicaron en las regiones frontal y parietal izquierdo al comparar CTRL y ESQ.
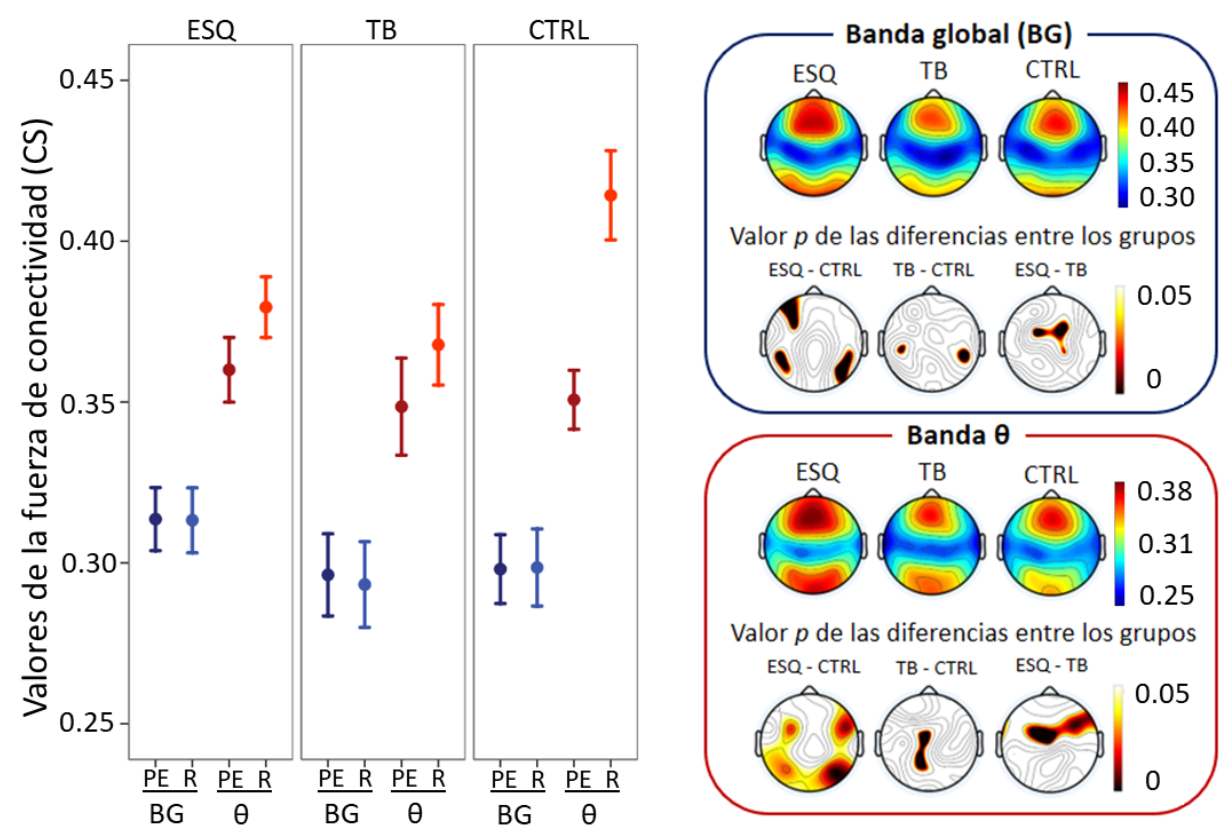

Fig. 11| Fuerza de conectividad (CS) de la red funcional. A la izquierda se muestran los intervalos de confianza del $95 \%$ de los valores de la CS en los grupos de estudio en la banda global (BG) y $\theta$, tanto en la ventana preestímulo $(\mathrm{PE})$ como respuesta $(\mathrm{R})$. A la derecha se representan los mapas topográficos de la distribución de los valores de la CS preestímulo en las bandas global y $\theta$, así como los valores $p$ de la diferencia al comparar los grupos. ESQ, pacientes con esquizofrenia; TB, pacientes con trastorno bipolar; CTRL, controles sanos. 

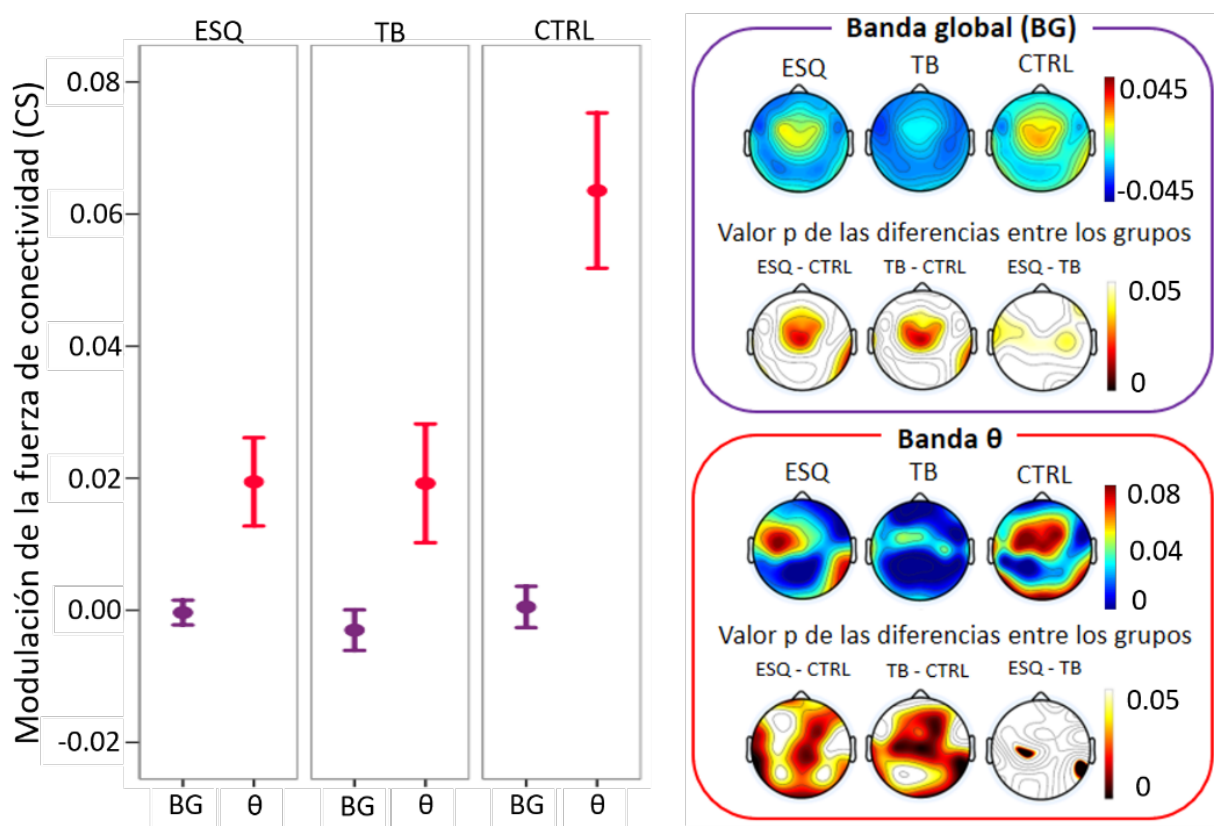

Fig. 12 Modulación de la fuerza de conectividad durante la tarea cognitiva. A la izquierda se muestran los intervalos de confianza del $95 \%$ de los valores de la modulación de la CS en los grupos de estudio en la banda global (BG) y $\theta$, definida como la diferencia entre la ventana preestímulo y respuesta durante una tarea cognitiva. A la derecha se representan los mapas topográficos de la distribución de los valores de la modulación de la CS en las bandas global y $\theta$, así como los valores $p$ de la diferencia al comparar los grupos. ESQ, pacientes con esquizofrenia; TB, pacientes con trastorno bipolar; CTRL, controles sanos.

En cuanto a la modulación en la banda $\theta$, las principales diferencias se ubicaron en regiones central y frontal al comparar CTRL y ambos grupos de pacientes.

- Relación entre la CS preestímulo en la banda global y la modulación de la CS en la banda $\boldsymbol{\theta}$. En los pacientes la diferencia fue significativa $(r=-0.24, \mathrm{n}=108, p=0.01)$ e indicó que los valores mayores de CS preestímulo se asociaron con aumentos menores en la modulación CS $\theta$. 


\subsubsection{Relación de las alteraciones de los parámetros de conectividad funcional con la duración de la enfermedad}

- CS preestímulo en la banda global. Al comparar el grupo EPE $($ media $=0.32, \mathrm{SD}=0.06)$ con $\mathrm{EC}($ media $=0.31, \mathrm{SD}=0.03)$ no hubo diferencias significativas $(t=1.04, p=0.29)$.

- Modulación CS en la banda $\boldsymbol{\theta}$. Al comparar el grupo EPE (media = $0.024, \mathrm{SD}=0.03)$ con $\mathrm{EC}($ media $=0.016, \mathrm{SD}=0.03)$ no hubo diferencias significativas $(t=1.14, p=0.25)$.

- No hubo correlación significativa entre la duración de la enfermedad (en meses) y la CS preestímulo en la banda global o la modulación de la CS en la banda $\theta$.

\subsubsection{Relación de las alteraciones de los parámetros de conectividad funcional con el tratamiento farmacológico}

- CS preestímulo en la banda global. No hubo diferencia significativa entre los pacientes TB tratados y no tratados con antipsicóticos $(U=84, \mathrm{z}=-0.19, p=0.86)$. Tampoco hubo diferencias significativas entre los pacientes TB tratados y no tratados con litio $(U=73, \mathrm{z}=-0.37, p=0.73)$, benzodiacepinas $(U$ $=481, \mathrm{z}=0.69, p=0.48)$, anticonvulsivos $(U=99, \mathrm{z}=0.28, p=$ $0.79)$ o antidepresivos $(U=201, \mathrm{z}=0.83, p=0.40)$. En el grupo ESQ no hubo diferencias significativas entre los participantes que recibieron o no benzodiacepinas $(U=84, \mathrm{z}=-0.293, p=0.79) \mathrm{o}$ antidepresivos $(U=60, \mathrm{z}=-1.08, p=0.29)$ (Fig. 13).

- Modulación CS en la banda $\boldsymbol{\theta}$. No hubo diferencias significativas entre los pacientes TB que recibieron y no recibieron antipsicóticos $(U=70, \mathrm{z}=-0.88, p=0.37)$. Tampoco hubo diferencias significativas entre pacientes TB que recibieron y no recibieron litio $(U=55, \mathrm{z}=-1.13, p=0.18)$, BZD $(U=220, \mathrm{z}=1.32, p=0.19)$, anticonvulsivos $(U=84, \mathrm{z}=-0.79, p=0.42)$ o antidepresivos $(U=$ $190, \mathrm{z}=-1.09, p=0.27)$. 
A

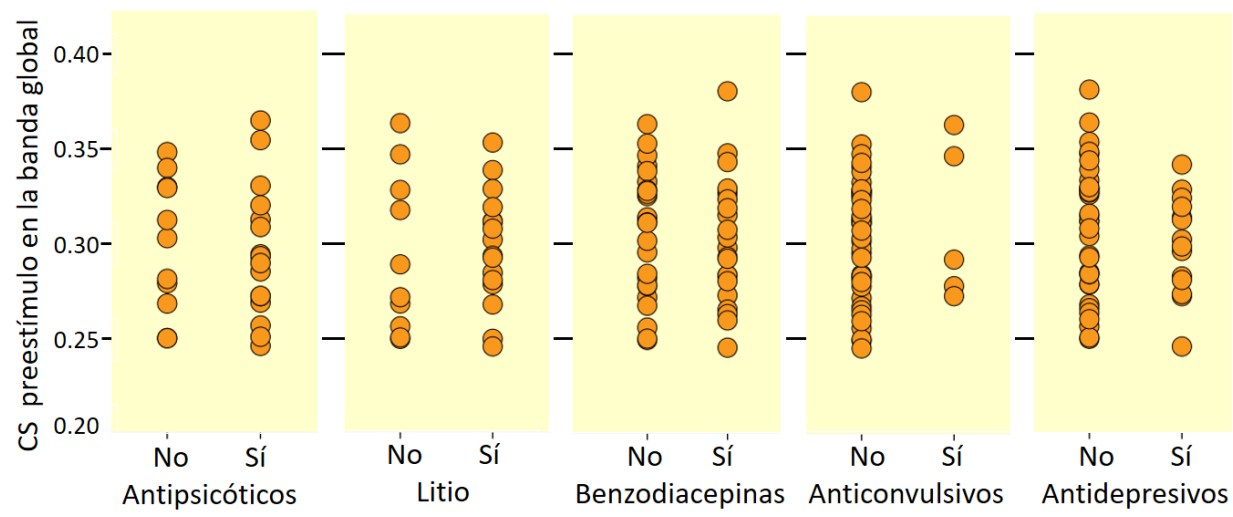

B

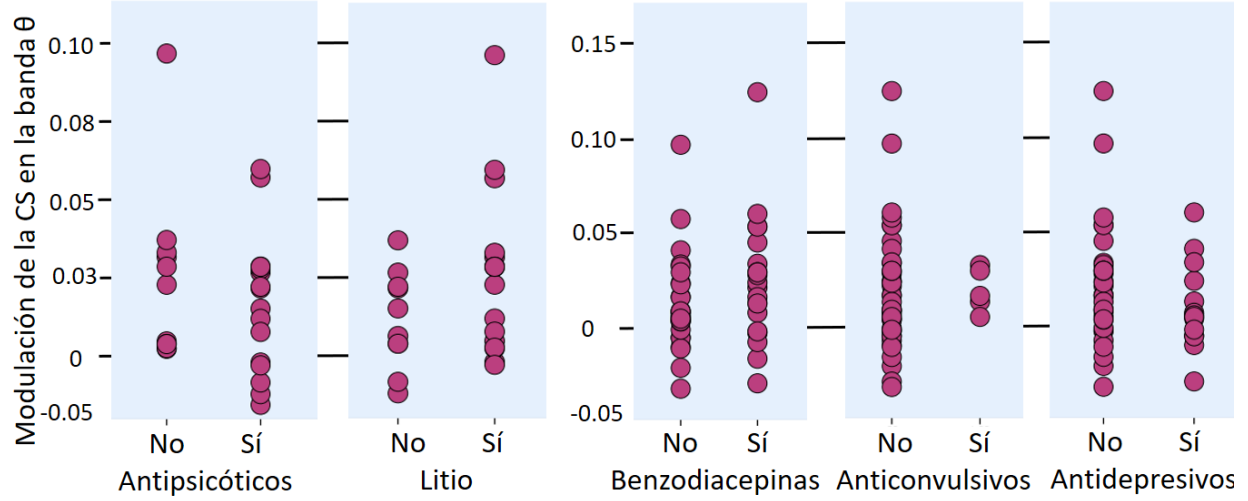

Fig. 13 Relación de la fuerza de conectividad y su modulación con el tratamiento farmacológico. (A) Distribuciones individuales de la fuerza de conectividad (CS) preestímulo en la banda global de los pacientes tratados y no tratados con los diferentes grupos de psicofármacos. (B) Distribuciones individuales de la modulación de la CS en la banda $\theta$ durante la realización de una tarea cognitiva de los pacientes tratados y no tratados con los diferentes grupos de psicofármacos. En ambos casos, en las comparaciones de antipsicóticos y litio se incluyeron solo a pacientes con trastorno bipolar y en las restantes a todos los pacientes.

En el grupo ESQ no hubo diferencias significativas entre los que recibieron o no BZD $(U=61, \mathrm{z}=-1.41, p=0.17)$ o antidepresivos $(U=59, \mathrm{z}=-1.13, p=0.27)$.

- Correlación entre la CS preestímulo en la banda global y la modulación en la banda $\theta$ con la dosis de antipsicótico. No hubo correlación significativa entre la dosis de antipsicóticos de todos los pacientes y la CS preestímulo en la banda global $(r=0.058, \mathrm{n}=94$, $p=0.58)$ ni con la modulación de la CS en la banda $\theta(r=-0.05, p$ 
$=0.53)$. Estas correlaciones tampoco fueron significativas en el grupo ESQ considerado por separado (CS preestímulo BG: $r=$ -0.004; modulación CS $\theta: r=-0.046$ ) ni en el grupo TB (CS preestímulo BG: $r=-0.025$; modulación CS $\theta r=-0.008$ ).

\subsubsection{Relación de las alteraciones de los parámetros de conectividad funcional con la cognición y los síntomas}

\section{Rendimiento cognitivo}

- El análisis de componentes principales de las puntuaciones cognitivas produjo un único factor que combinaba todas las puntuaciones individuales y explicaba el $55.40 \%$ de la varianza (valor propio 3.34). Hubo diferencias significativas del valor del factor entre los grupos, que fue menor en los pacientes en comparación con CTRL, lo que implica un peor rendimiento cognitivo general.

- En el grupo ESQ la regresión lineal múltiple seleccionó el CS preestímulo en la banda global como un predictor significativo de la cognición general $\left(\mathrm{R}^{2}=0.137, \mathrm{~F}=8.70, p=0.005\right)$, que se asoció con un peor rendimiento cognitivo (Fig. 14).

- En el grupo TB no hubo predictores significativos de la cognición general.

\section{Síntomas}

- En el grupo ESQ la regresión lineal múltiple seleccionó la modulación CS en la banda $\theta$ como un predictor significativo de síntomas negativos $\left(\mathrm{R}^{2}=0.084, \mathrm{~F}=6.23, p=0.015\right)$, pero no hubo predictores significativos de los síntomas positivos.

- En el grupo TB, no hubo correlación significativa entre los valores de CS y las puntuaciones de la PANSS.

- En todos los pacientes, no hubo correlación significativa entre los valores de CS y las puntuaciones de las escalas HDRS e YMRS. 
A

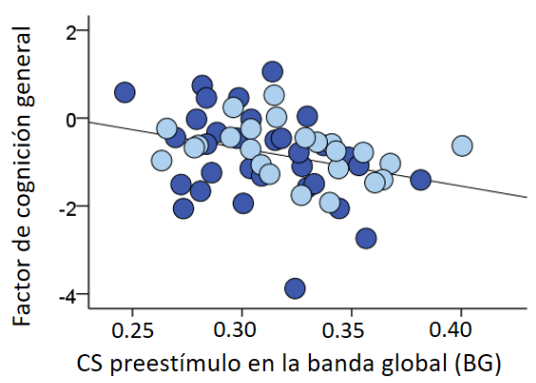

B

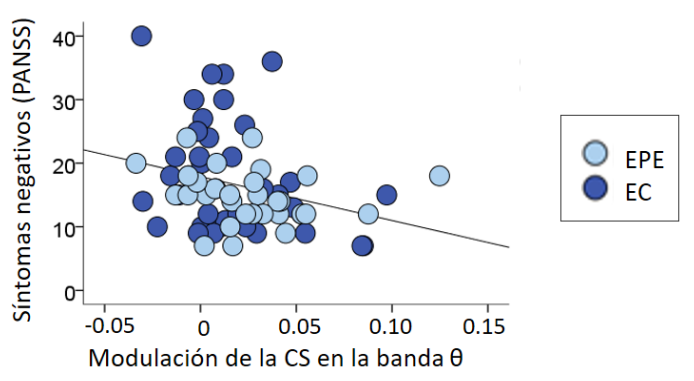

Fig. 14| Relación de la fuerza de conectividad con las puntuaciones cognitivas y los síntomas en la esquizofrenia. (A) Diagrama de dispersión que muestra la correlación inversa entre los valores de fuerza de conectividad (CS) en la ventana preestímulo en la banda global y los valores del factor de cognición general que resume las puntuaciones cognitivas. (B) Diagrama de dispersión que muestra la correlación inversa entre los valores de la modulación de la CS en la banda $\theta$ y las puntuaciones de síntomas negativos de la Escala para el síndrome positivo y negativo de la esquizofrenia (PANSS). EPE, pacientes con esquizofrenia en el primer episodio; EC, pacientes con esquizofrenia crónica.

\subsection{Potencia de ruido cortical en la banda y (NP-y)} Artículo 3: Altered gamma band noise power in schizophrenia and bipolar patients during a cognitive task.

\subsubsection{Resultados demográficos, clínicos y cognitivos}

- Distribución por sexo. No hubo diferencias significativas entre los grupos $\left(\chi^{2}=0.041, p=0.98\right)$ (Tabla 6).

- Distribución por edad. Hubo diferencias significativas entre los grupos: el grupo TB era mayor en comparación con CTRL (diferencia de medias $=5.63, p=0.04$ ).

Escalas de valoración cognitiva. Hubo diferencias significativas entre los grupos: En comparación con CTRL, los pacientes mostraron puntuaciones menores en el coeficiente intelectual total y en todos los dominios BACS, excepto la torre de Londres. En cuanto al desempeño durante la tarea oddball, los pacientes tuvieron peores tiempos de reacción, dieron menos respuestas correctas y cometieron más errores por omisión y falsas alarmas. 


\section{Tabla 6 | Resultados demográficos, cognitivos, clínicos y del tratamiento en el estudio de la potencia de ruido cortical en la banda y}

\begin{tabular}{|c|c|c|c|}
\hline & $\begin{array}{c}\text { Pacientes con } \\
\text { esquizofrenia } \\
\text { (ESQ) } \\
\text { N = 29 }\end{array}$ & $\begin{array}{l}\text { Pacientes con } \\
\text { trastorno bipolar } \\
\text { (TB) } \\
\text { N }=27\end{array}$ & $\begin{array}{l}\text { Controles } \\
\text { sanos } \\
\text { (CTRL) } \\
\mathrm{N}=36\end{array}$ \\
\hline \multicolumn{4}{|l|}{ Datos demográficos } \\
\hline $\begin{array}{l}\text { Sexo } \\
\text { (hombre: mujer) }\end{array}$ & $19: 10$ & $17: 10$ & $23: 13$ \\
\hline Edad (años) & $41.00(8.20)$ & $45.52(9.91)^{\star}$ & $39.89(8.53)$ \\
\hline \multicolumn{4}{|l|}{ Escalas cognitivas } \\
\hline WAIS-III Cl total & $94.41(11.94)^{* *}$ & $98.05(9.93)^{\star *}$ & $115.65(8.80)$ \\
\hline BACS & $33.61(11.87)^{\star *}$ & $35.63(9.19)^{\star *}$ & $49.21(8.02)$ \\
\hline -Memoria verbal & $33.61(11.87)^{* *}$ & $35.63(9.19)^{\star *}$ & $49.21(8.02)$ \\
\hline -Memoria de trabajo & $15.36(4.11)^{\star *}$ & $17.47(3.85)^{\star *}$ & $22.26(3.05)$ \\
\hline -Velocidad motora & $59.78(19.55)^{* *}$ & $66.53(13.40)^{* *}$ & $80.59(11.90)$ \\
\hline -Fluidez verbal & $17.04(5.34)^{\star \star}$ & $20.79(5.90)^{\star \star}$ & $28.70(6.29)$ \\
\hline $\begin{array}{l}\text {-Rapidez en el } \\
\text { procesamiento }\end{array}$ & $37.61(12.82)^{\star *}$ & $41.53(12.32)^{\star *}$ & $64.50(10.81)$ \\
\hline -Funciones ejecutivas & $16.46(4.734)$ & $16.26(2.978)$ & $17.82(3.27)$ \\
\hline Factor cognitivo general 1 & $-0.82(0.79)^{* *}$ & $-0.43(0.63)^{* *}$ & $0.87(0.51)$ \\
\hline Factor cognitivo general 2 & $-0.03(1.15)$ & $-0.16(0.82)$ & $0.11(0.98)$ \\
\hline Datos clínicos & & & N/A \\
\hline PANSS-P positiva & $12.04(4.59)^{\star *}$ & $7.33(0.84)$ & \\
\hline PANSS-N negativa & $18.20(8.25)^{\star \star}$ & $8.94(2.51)$ & \\
\hline PANSS totall & $57.16(19.48)^{\star \star}$ & $30.33(3.24)$ & \\
\hline $\begin{array}{l}\text { Duración de la enfermedad } \\
\text { (años) }\end{array}$ & $173.57(105.53)$ & $193.91(105.23)$ & \\
\hline Tratamiento (N) & & & N/A \\
\hline $\begin{array}{l}\text { Dosis de antipsicótico } \\
\text { (Eq de CPZ mg/d) }\end{array}$ & $416.46(203.52)^{\star \star}$ & $230.78(123.77)$ & \\
\hline Antipsicóticos & 29 & 16 & \\
\hline Litio & 0 & 17 & \\
\hline Benzodiacepinas & 17 & 12 & \\
\hline Anticonvulsivos & 0 & 14 & \\
\hline Antidepresivos & 8 & 11 & \\
\hline \multicolumn{4}{|l|}{ Neurofisiología } \\
\hline P3b amplitud Pz $(\mu \mathrm{V})$ & $1.22(1.85)$ & $1.56(1.71)$ & $1.780(2.02)$ \\
\hline Ensayos diana incluidos & $75.50(8.12)^{* *}$ & $84.04(19.57)$ & $93.69(12.20)$ \\
\hline Respuestas correctas (\%) & $83.46(18.83)^{* *}$ & $90.82(11.11)^{* *}$ & $99.23(0.99)$ \\
\hline Omisiones (\%) & $16.54(18.83)^{\star *}$ & $9.18(11.11)^{* *}$ & $0.77(0.99)$ \\
\hline Falsas alarmas (\%) & $15.57(18.57)^{* *}$ & $12.87(16.80)^{* *}$ & $1.43(1.97)$ \\
\hline Tiempo de reacción (ms) & $298.49(46.87)^{\star *}$ & $276.68(58.75)^{\star \star}$ & $241.13(41.84)$ \\
\hline
\end{tabular}

Para la edad, las escalas cognitivas, los datos clínicos y de neurofisiología se muestran los valores de la media y desviación estándar (SD). Se representa la significación estadística de la comparación entre los pacientes y CTRL o la comparación entre los pacientes. ${ }^{*} p<0.05 ;{ }^{* *} p<0.01$. WAIS-III, Escala de inteligencia del adulto de Wechsler; CI, cociente intelectual; BACS, Escala de evaluación breve de la cognición en la esquizofrenia; PANSS, Escala para el síndrome positivo y negativo de la esquizofrenia; Eq. de CPZ, equivalentes de clorpromazina; N/A, no es aplicable. 
- Duración de la enfermedad. No hubo diferencias significativas entre los grupos de pacientes.

- Tratamiento farmacológico. Hubo diferencias significativas entre los grupos: el grupo ESQ había recibido dosis más altas de antipsicóticos comparado con TB $(t=3.307, p=0.002)$.

\subsubsection{Especificidad para el diagnóstico de las alteraciones de la potencia de ruido (NP-y)}

- Las comparaciones no paramétricas de la NP- $\gamma$ corregidas por Bonferroni mostraron diferencias significativas entre los grupos (Tabla 7 y Fig. 15).

A

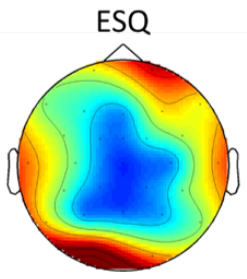

B ESQ - CTRL

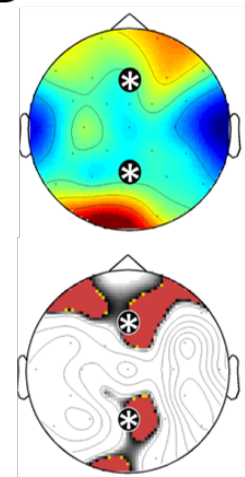

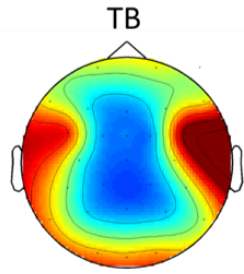

TB - CTRL
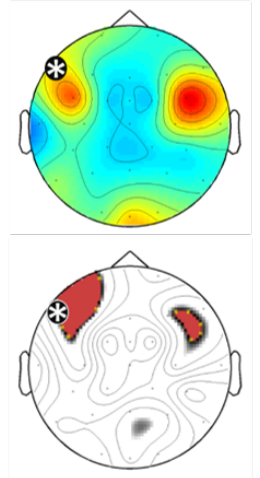

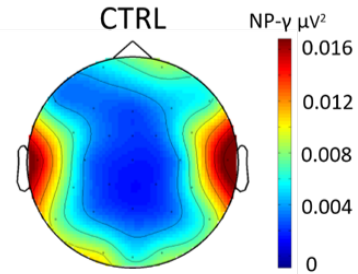

ESQ - TB
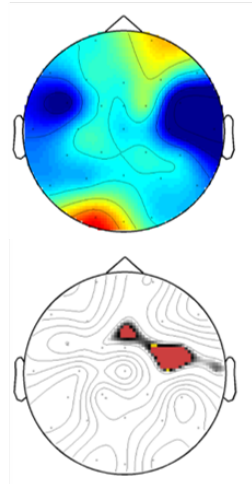

$N P-\gamma \mu V^{2}$

0.012

0.008

0.004

0

$-0.002$

-0.00
0.05

0.04

0.03

0.02

0.01

Fig. 15 Potencia de ruido en la banda y en la esquizofrenia y el trastorno bipolar. (A) Los mapas topográficos muestran los promedios generales de los valores de la NP-y $\left(e n \mu V^{2}\right)$ de cada electrodo del EEG en los grupos de participantes. (B) En la fila superior los mapas representan las diferencias de la NP-y al comparar los grupos y en la fila inferior se muestra la significación estadística de estas comparaciones (valor $p$ ); los electrodos que mostraron diferencias significativas después de aplicar la corrección de Bonferroni se señalan con un asterisco. ESQ, pacientes con esquizofrenia; TB, pacientes con trastorno bipolar; CTRL controles sanos. 
Tabla 7 | Valores de la potencia de ruido cortical en la banda y (NP- - )

\begin{tabular}{|c|c|c|c|c|c|c|}
\hline \multirow[t]{2}{*}{$N P-Y$} & \multicolumn{2}{|c|}{$\begin{array}{c}\text { Pacientes con } \\
\text { esquizofrenia (ESQ) } \\
\text { N }=29\end{array}$} & \multicolumn{2}{|c|}{$\begin{array}{l}\text { Pacientes con } \\
\text { trastorno bipolar } \\
\text { (TB) } \\
N=27\end{array}$} & \multicolumn{2}{|c|}{$\begin{array}{l}\text { Controles sanos } \\
\qquad \begin{array}{c}\text { (CTRL) } \\
\text { N }=36\end{array}\end{array}$} \\
\hline & Media & SD & Media & SD & Media & SD \\
\hline Fp1 & $0.00967^{\#}$ & $(0.01026)$ & $0.00868^{\#}$ & $(0.00665)$ & 0.00534 & $(0.00506)$ \\
\hline Fp2 & $0.01509^{\#}$ & $(0.01557)$ & 0.00934 & $(0.00811)$ & 0.00814 & $(0.00922)$ \\
\hline F7 & $0.00780^{\#}$ & $(0.00967)$ & $0.00936^{\#}$ & $(0.01153)$ & 0.00394 & $(0.00304)$ \\
\hline F3 & $0.00600^{\#}$ & $(0.00689)$ & $0.00508^{\#}$ & $(0.00345)$ & 0.00315 & $(0.00219)$ \\
\hline $\mathrm{Fz}$ & $0.00384^{\star \#}$ & $(0.00200)$ & 0.00259 & $(0.00161)$ & 0.00230 & $(0.00160)$ \\
\hline F4 & $0.01044^{\#}$ & $(0.01720)$ & 0.00738 & $(0.00669)$ & 0.00462 & $(0.00433)$ \\
\hline F8 & 0.01003 & $(0.00953)$ & 0.00989 & $(0.00872)$ & 0.00634 & $(0.00821)$ \\
\hline FC5 & 0.00794 & $(0.01029)$ & $0.01438^{\#}$ & $(0.01528)$ & 0.00635 & $(0.00773)$ \\
\hline FC1 & 0.00388 & $(0.00290)$ & 0.00282 & $(0.00159)$ & 0.00276 & $(0.00367)$ \\
\hline $\mathrm{FCz}$ & $0.00311^{\#}$ & $(0.00141)$ & 0.00243 & $(0.00179)$ & 0.00215 & $(0.00170)$ \\
\hline FC2 & $0.00583^{\star \#}$ & $(0.00699)$ & 0.00271 & $(0.00146)$ & 0.00263 & $(0.00252)$ \\
\hline FC6 & $0.00880^{*}$ & $(0.00945)$ & $0.01850^{\#}$ & $(0.02294)$ & 0.00860 & $(0.01041)$ \\
\hline $\mathrm{T7}$ & 0.01244 & $(0.01790)$ & 0.01527 & $(0.01167)$ & 0.01605 & $(0.01760)$ \\
\hline C3 & 0.00715 & $(0.01447)$ & 0.00636 & $(0.00826)$ & 0.00374 & $(0.00487)$ \\
\hline $\mathrm{Cz}$ & 0.00185 & $(0.00099)$ & 0.00179 & $(0.00088)$ & 0.00167 & $(0.00088)$ \\
\hline C4 & $0.00356^{*}$ & $(0.00323)$ & 0.00952 & $(0.01600)$ & 0.00430 & $(0.00610)$ \\
\hline T8 & $0.01224^{*}$ & $(0.01862)$ & 0.02270 & $(0.01837)$ & 0.01843 & $(0.01808)$ \\
\hline CP5 & 0.00749 & $(0.01041)$ & 0.00750 & $(0.00778)$ & 0.00571 & $(0.00816)$ \\
\hline CP1 & $0.00320^{\#}$ & $(0.00325)$ & 0.00199 & $(0.00130)$ & 0.00185 & $(0.00148)$ \\
\hline CP2 & 0.00324 & $(0.00419)$ & 0.00204 & $(0.00116)$ & 0.00179 & $(0.00108)$ \\
\hline CP6 & 0.00731 & $(0.01119)$ & 0.00765 & $(0.00672)$ & 0.00698 & $(0.01144)$ \\
\hline P7 & 0.01078 & $(0.01663)$ & 0.01138 & $(0.01366)$ & 0.00818 & $(0.00855)$ \\
\hline P3 & 0.00363 & $(0.00306)$ & 0.00421 & $(0.00434)$ & 0.00322 & $(0.00319)$ \\
\hline $\mathrm{Pz}$ & $0.00276^{\#}$ & $(0.00178)$ & 0.00222 & $(0.00150)$ & 0.00175 & $(0.00086)$ \\
\hline P4 & 0.00415 & $(0.00432)$ & 0.00368 & $(0.00238)$ & 0.00290 & $(0.00210)$ \\
\hline P8 & 0.00787 & $(0.00884)$ & 0.00861 & $(0.00675)$ & 0.00753 & $(0.00884)$ \\
\hline 01 & $0.02057^{\#}$ & $(0.02518)$ & 0.01160 & $(0.01323)$ & 0.00909 & $(0.01025)$ \\
\hline $\mathrm{Oz}$ & $0.01721^{\#}$ & $(0.02282)$ & 0.01180 & $(0.02045)$ & 0.00604 & $(0.00813)$ \\
\hline $\mathrm{O} 2$ & 0.01213 & $(0.01244)$ & 0.01160 & $(0.01790)$ & 0.00729 & $(0.01599)$ \\
\hline
\end{tabular}

Se muestran los valores de la media y desviación estándar (SD). Se representa la significación estadística de la comparación entre los pacientes y controles $\left({ }^{*} p<0.05\right)$ y la comparación entre pacientes con esquizofrenia y trastorno bipolar $(\# p<0.05)$. Se resalta en letra negrilla las comparaciones significativas tras la corrección de Bonferroni $(p<0.002)$.

- En comparación con el grupo CTRL, el grupo ESQ mostró valores más altos en los electrodos $\mathrm{Fz}(t=3.460, p=0.002)$ y $\mathrm{Pz}(t=2.997$, $p=0.002)$ y el grupo TB mostró valores más altos en el electrodo $\mathrm{F} 7$ $(t=2.702, p=0.002)$.

- No hubo diferencias significativas al comparar ESQ con TB 


\subsubsection{Relación de las alteraciones de la NP-y con la duración de la enfermedad}

No hubo correlación significativa entre la duración de la enfermedad y los valores de la NP- $\gamma$ alterados en ESQ o TB.

\subsubsection{Relación de las alteraciones de la NP- $\gamma$ con el tratamiento farmacológico}

- En el grupo ESQ no hubo correlación significativa entre los valores alterados de la NP- $\gamma$ y las dosis de antipsicóticos (Fig. 16).

- En los electrodos con valores alterados de la NP- $\gamma$ no hubo diferencias significativas entre los pacientes tratados y no tratados con los diferentes grupos de fármacos (la comparación para los antipsicóticos y litio se hizo en el grupo TB, y los restantes en todos los pacientes) (Fig. 16).

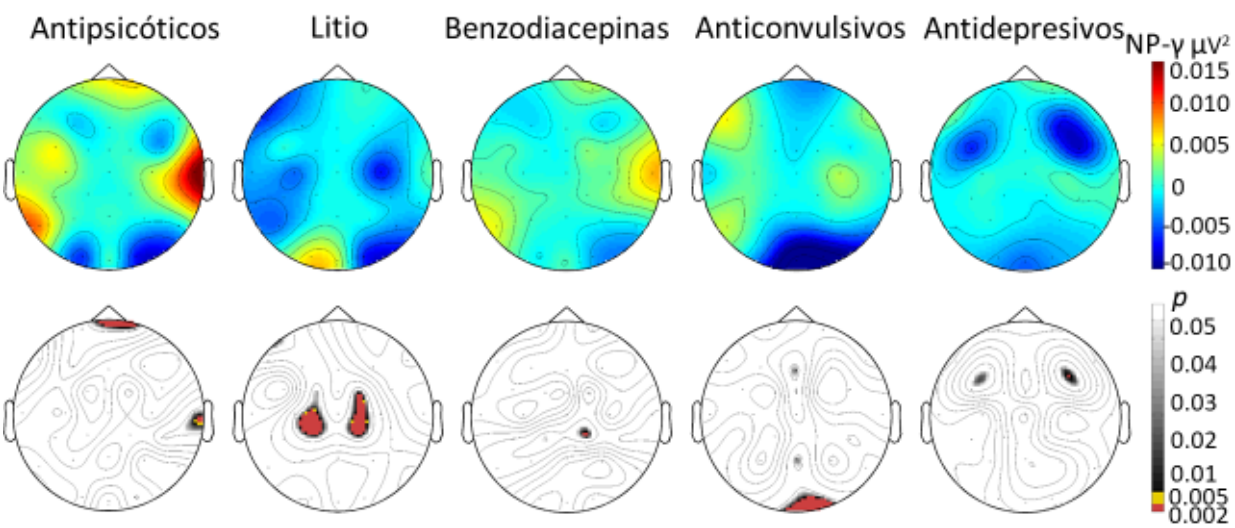

Fig. 16|. Relación de la potencia de ruido en la banda y y el tratamiento farmacológico. En la fila superior los mapas topográficos representan los valores de la diferencia de la NP-y al comparar pacientes tratados y no tratados con diferentes tipos de psicofármacos. En la fila inferior, los mapas topográficos representan la significación estadística de estas comparaciones (valor $p$ ) en las que ningún electrodo mostró diferencias significativas después de aplicar la corrección de Bonferroni. 


\subsubsection{Relación de las alteraciones de la NP-y con la cognición y los síntomas}

\section{Rendimiento cognitivo}

- El análisis de componentes principales de las puntuaciones BACS produjo dos factores independientes (valor propio $>1$ ) que explicaron respectivamente el 57,4\% y el 17,6\% de la varianza. El primer factor cognitivo incluyó todas las puntuaciones BACS excepto el dominio de funciones ejecutivas/resolución de problemas (torre de Londres), que correspondió al segundo factor cognitivo. Hubo diferencias significativas entre los grupos de las puntuaciones del primer factor cognitivo, que fueron menores en ambos grupos de pacientes en comparación con CTRL $(p<0.001)$.

- No hubo correlación significativa entre los valores alterados de la NP $-\gamma$ y las puntuaciones de los factores cognitivos en ninguno de los grupos de pacientes.

- No hubo correlación significativa entre los valores alterados de la NP- $\gamma$ y ninguna de las variables de rendimiento en la tarea oddball.

\section{Escalas de síntomas}

- En el grupo ESQ no hubo correlación significativa entre los valores alterados de la NP- $\gamma$ y las puntuaciones de PANSS, aunque después de la corrección de Bonferroni se observó una correlación significativa entre la puntuación del síndrome negativo de la PANSS y otros electrodos F7 $(\rho=0.642, p=0.001)$, FC5 $(\rho=0.612, p=$ $0.001), \mathrm{T} 7(\rho=0.649, p<0.001), \mathrm{P} 7(\rho=0.627, p=0.001), \mathrm{P} 3(\rho=$ $0.629, p=0.001)$, P4 $(\rho=0.581, p=0.002)$ y P8 $(\rho=0.712, p<$ $0.001)$. Así también hubo correlación significativa entre la puntuación total de la PANSS y los electrodos T7 $(\rho=0.668, p<$ $0.001)$, P7 ( $\rho=0.665, p<0.001)$ y P8 $(\rho=0.618, p=0.001)$ (Fig. 18).

- En el grupo TB no hubo correlación entre los valores alterados de la NP- $\gamma$ y las puntuaciones de la PANSS.

En el grupo ESQ los modelos de regresión lineal múltiple no revelaron predictores significativos del rendimiento cognitivo ni de las puntuaciones en las escalas de síntomas. 

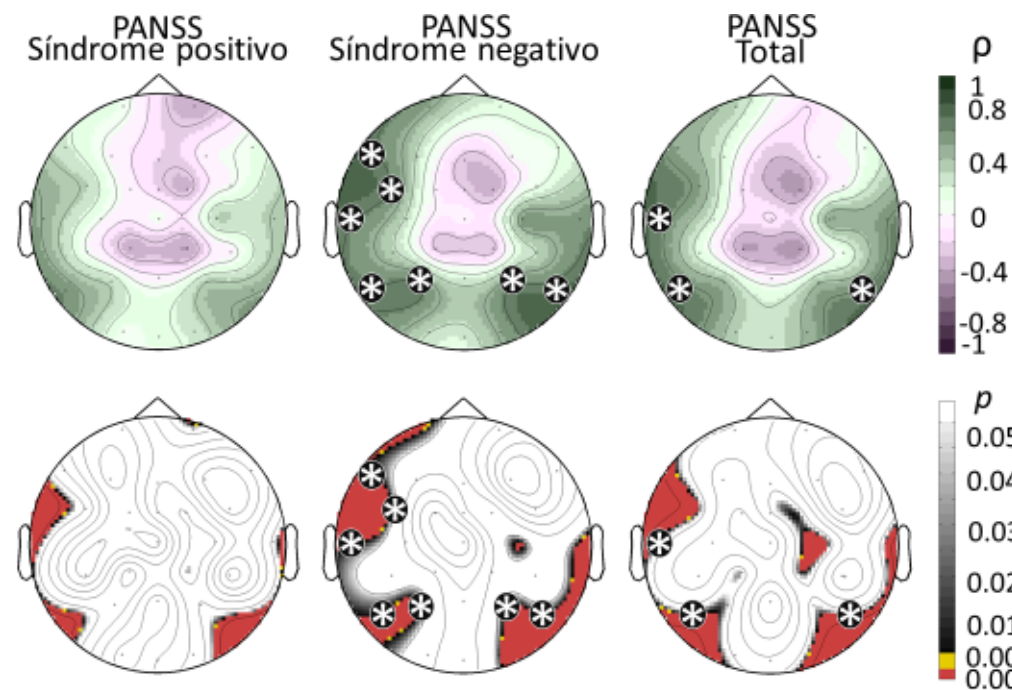

0.05

0.04

0.03

0.02

0.01

0.005

Fig. 17| Relación de la potencia de ruido en la banda y y los síntomas en los pacientes con esquizofrenia. En la fila superior los mapas topográficos representan los valores de $\rho$ de la correlación entre los valores de NP-y y los síntomas evaluados por la Escala del síndrome positivo y negativo en la esquizofrenia (PANSS). En la fila inferior se representa la significación estadística (valor $p$ ) de esta correlación, que fue significativa en los electrodos de la región frontal izquierda y temporoparietal bilateral (señalada con asteriscos) para las puntuaciones de síntomas negativos y total. 


\section{CAPÍTULO 5}

\section{Discusión}

as herramientas y métodos para estudiar el funcionamiento del cerebro humano y sus alteraciones han experimentado un gran

avance en las últimas décadas, produciendo un considerable incremento en el tipo y volumen de datos disponibles. En este sentido, la caracterización de redes cerebrales estructurales y funcionales mediante la teoría de redes complejas, permite disponer de modelos útiles para comprender la organización y el funcionamiento cerebral, al mismo tiempo que propone nuevos caminos para abordar los posibles mecanismos etiopatogénicos de distintas enfermedades mentales.

El parámetro de conectividad de la red cerebral utilizado en esta tesis para caracterizar la conectividad cerebral en la esquizofrenia y el trastorno bipolar es la fuerza de conectividad (CS) y en el caso de la conectividad estructural, también el índice de mundo pequeño (SW). Por otra parte, la conectividad de las redes cerebrales funcionales se fundamenta en la actividad rítmica oscilatoria de las asambleas neuronales que la forman, por lo que se estudia la actividad oscilatoria cortical en la banda $\gamma$ a través de la estimación de la potencia del ruido cortical (NP). Estos parámetros fueron estimados para realizar comparaciones directas que ayuden a determinar su especificidad para el diagnóstico y conocer su posible relación con la duración de la enfermedad y con el tratamiento farmacológico. La discusión de los resultados se presenta en tres secciones correspondientes a cada uno de los artículos de investigación. 


\subsection{Conectividad estructural}

Según los resultados, la CS y el SW de la red estructural están reducidos tanto en la esquizofrenia crónica como en el trastorno bipolar, pero no en la esquizofrenia en el primer episodio psicótico. Las alteraciones del índice SW probablemente son el resultado de valores más altos de la longitud de camino característica $(L)$ de la red. Estos hallazgos son consistentes con publicaciones previas en la esquizofrenia $(2,245)$.

No se han encontrado estudios de conectividad estructural que hagan comparaciones directas entre la esquizofrenia y el trastorno bipolar utilizando parámetros de la teoría de grafos basados en datos de anisotropía fraccional (FA) (245), si bien un estudio de conectividad estructural basado en las correlaciones de los valores regionales de espesor de materia gris — un índice indirecto de conectividad corticalencontró alteraciones en la esquizofrenia deficitaria pero no en la esquizofrenia no deficitaria ni en el trastorno bipolar (209). Asimismo, una revisión de estudios que utilizan la teoría de grafos y la imagen de difusión para evaluar la conectividad estructural por separado en la esquizofrenia y el trastorno bipolar respalda la noción de desconectividad frontal en ambos trastornos (6).

La estimación de la conectividad estructural en esta tesis se basa en valores de FA, que dependen de la integridad axonal y de la mielina, de modo que también es posible que haya otros factores (destrucción axonal o desmielinización) que podrían contribuir potencialmente a la reducción de la FA (246). No obstante, los datos aquí obtenidos en los pacientes con esquizofrenia crónica son consistentes con una menor FA informada en la esquizofrenia $(247,248)$, así como en la esquizofrenia y el trastorno bipolar (249). Asimismo, la mayor $L$ en los pacientes con esquizofrenia crónica también es consistente con la reducción de las vías de comunicación global descrita en este trastorno $(250,251)$. Más aun, las alteraciones de la red en el trastorno bipolar también son acordes con informes previos sobre la reducción generalizada de la FA en este síndrome (252). Tomados en conjunto, todos estos hallazgos sugieren la presencia de una afectación de los tractos de sustancia blanca en las psicosis crónicas. 
Debe tenerse en cuenta que el parámetro que valora la arquitectura conectiva (SW) fue calculado en la red cerebral global, sin considerar las posibles alteraciones circunscritas a regiones específicas. No obstante, algunas investigaciones sugieren una distribución regional desigual de las alteraciones de la conectividad en la esquizofrenia y en el trastorno bipolar. La disminución del SW de la red global en la esquizofrenia podría estar relacionada con la reducción de la centralidad de intermediación (betweenness centrality) de los hubs frontales que ha sido informada en la esquizofrenia (2) y que ha sido descrita también en investigaciones de redes basadas en imágenes funcionales $(251,253)$. Un estudio reciente basado en volúmenes de materia gris respalda la evidencia de hiperconectividad entre la red neuronal por defecto (DMN) y la red límbica en la esquizofrenia en comparación con el trastorno bipolar, así como la hipoconectividad entre la red límbica y la de atribución de relevancia (salience network) (254). Además, se han informado alteraciones de la FA en las conexiones entre la corteza prefrontal, el núcleo caudado, el tálamo y el cingulado anterior en la esquizofrenia (233), que parecen consistentes con esos resultados. Otro estudio con DTI del cuerpo calloso y haz del cíngulo en pacientes con esquizofrenia, trastorno bipolar y controles no encontró diferencias de FA entre los grupos, aunque encontró mayor difusividad radial (RD) y coeficiente de difusión aparente (ADC) solo en los pacientes con esquizofrenia (255). Estas diferencias regionales de la conectividad pueden ser la base de las alteraciones en los patrones de conectividad global observadas en ambos trastornos psicóticos.

Las alteraciones de la conectividad estructural encontradas no parecen estar relacionadas con el tratamiento antipsicótico, ya que no hubo correlación entre los parámetros de la red y la dosis de antipsicóticos recibida, ni estos parámetros difirieron entre los pacientes bipolares que recibieron o no el tratamiento antipsicótico. Por otra parte, la cronicidad de la enfermedad sí podría tener un efecto perjudicial progresivo en la conectividad, según lo sugiere la relación inversa encontrada en este estudio entre la duración de la enfermedad (pero no la edad) y el SW en los pacientes. De esta manera, de acuerdo a los resultados obtenidos, la duración de la enfermedad, pero no el tratamiento antipsicótico, podría estar relacionada con la desconectividad de la red global en estos síndromes. 
En el trastorno bipolar las alteraciones en la conectividad podrían estar relacionadas de manera hipotética con otros tratamientos distintos a los antipsicóticos — como los estabilizadores del estado de ánimo-, pero esto parece poco probable, ya que se ha informado una relación positiva entre la exposición al litio y los valores de FA (256), y la mayoría de los pacientes con trastorno bipolar incluidos en el estudio que tomaban litio.

En la esquizofrenia, los valores más altos de SW se asociaron con un mejor desempeño en tareas ejecutivas y cognición social (torre de Londres y WCST), lo que parece consistente con la amplia participación cortical en este tipo de tareas, dadas las múltiples demandas cognitivas subyacentes. Estos resultados parecen indicar que en la esquizofrenia las alteraciones de la arquitectura (SW) tienen un mayor impacto en estos dominios de la cognición que los déficits cuantitativos (CS) y que el deterioro de la arquitectura conectiva podría disminuir el rendimiento cognitivo, debido a una menor capacidad para armonizar la actividad de las distintas áreas involucradas. En el trastorno bipolar, sin embargo, no se detectó una relación similar entre la estructura de la red y la cognición. Esto podría estar relacionado con el tamaño de muestra más pequeño de este grupo, pero también puede sugerir diferentes fundamentos para el menor SW en el trastorno bipolar y la esquizofrenia, lo que se refuerza por la relación inversa entre el SW y los síntomas positivos que fue encontrada en el trastorno bipolar, pero no en la esquizofrenia.

\subsection{Conectividad funcional}

Los resultados muestran (i) valores de CS en la ventana preestímulo de la banda global (CS preestímulo BG) más altos en la esquizofrenia en comparación con el trastorno bipolar y el grupo control, y (ii) un déficit significativo de la modulación de los valores de CS durante la tarea cognitiva en la banda $\theta$ (modulación CS $\theta$ ) tanto en la esquizofrenia como en el trastorno bipolar.

La mayor CS preestímulo en la banda global encontrada en la esquizofrenia es conforme con los resultados previos publicados con muestras parcialmente superpuestas $(109,216)$. Respecto a estos, se 
replicó el hallazgo de una mayor CS preestímulo en la banda global (216) pero no en la banda $\theta(109)$.

En el análisis de la distribución espacial de la CS se encontró que la mayor CS preestímulo en la banda global no parece depender de la región, aunque se observaron diferencias significativas más extendidas al comparar el grupo de esquizofrenia con el grupo control. Por lo tanto, los resultados del estudio sugieren que el aumento de la CS preestímulo en la banda global puede ser específico de la esquizofrenia.

La CS se calculó a partir de los valores de acoplamiento de fase (PLV) entre los electrodos de registro del EEG, que a su vez se relacionan con la sincronía de fase de la actividad de las neuronas que forman las asambleas neuronales funcionales. Así, la mayor CS preestímulo en la banda global en la esquizofrenia (es decir valores promedio de PLV más altos entre los electrodos) sugiere un estado de hipersincronización de la actividad eléctrica en este síndrome que podría coincidir con el déficit de transmisión gabaérgica que se ha encontrado en este trastorno (31). La correlación inversa entre la CS preestímulo en la banda global y la cognición sugiere que esta hipersincronización dificulta potencialmente el procesamiento cognitivo.

La diferencia más notable entre los pacientes y controles fue la menor modulación de la CS en la banda $\theta$ —es decir, una reducción de la diferencia entre la CS de las ventanas preestímulo y respuesta- que fue común a la esquizofrenia y el trastorno bipolar. Esta reducción en la modulación puede interpretarse como un aumento general más pequeño o una hiposincronización de la actividad oscilatoria $\theta$ en los pacientes durante la realización de la tarea cognitiva. Este hallazgo concuerda con resultados anteriores que han mostrado una menor modulación de la frecuencia media (MF) y un menor aumento de la potencia relativa (RP) en la banda $\theta$ durante la realización de la misma tarea cognitiva en una muestra diferente de pacientes con esquizofrenia $(217,218)$.

No se descarta una relación entre el aumento en la CS preestímulo en la banda global y la reducción de la modulación en la banda $\theta$, sugerida por una correlación significativa en los pacientes. En otras palabras, no se descarta la posibilidad de que en los pacientes la actividad cerebral de la banda global hipersincronizada durante la ventana preestímulo pueda modificar la respuesta esperada durante la realización de la tarea. En condiciones normales la actividad $\theta$ aumenta durante la 
realización de la tarea cognitiva (257), de modo que la hipersincronización basal podría interferir con el patrón de activación cerebral esperado. Este planteamiento se ajusta con la proposición de la importancia que tiene el estado basal o de reposo del cerebro para los patrones de actividad cerebral inducidos por estímulos (258).

Además, se sabe que la frecuencia dominante de la actividad rítmica de las asambleas neuronales depende del número de neuronas participantes - bajas frecuencias implican asambleas neuronales de gran tamaño (22) - y que la actividad oscilatoria $\theta$ pueden sustentar interacciones cortico-corticales que comunican regiones relativamente distantes $(259,260)$. En este contexto, los hallazgos de una mayor CS preestímulo en la banda global y una menor modulación en la banda $\theta$ pueden significar una menor capacidad para la integración general de la información en las psicosis y podría explicar la relación de una menor modulación con los síntomas negativos en la esquizofrenia.

Como se ha indicado antes, estos valores de CS representan estimaciones de la red cerebral global. El análisis regional, por su parte, respalda la idea de que algunas regiones podrían tener un papel más prominente en el aumento de la CS. En la Fig. 12 se muestra que el déficit de modulación CS en la banda global es más evidente en las regiones centrales y frontales. Estas áreas coinciden o se superponen en parte con las de la DMN, la cual según algunas investigaciones presenta un estado basal hiperactivo y una menor desactivación relacionada con la tarea en pacientes con esquizofrenia $(261,262)$ y con trastorno bipolar $(263)$ en comparación con controles sanos. Por lo tanto, los resultados obtenidos apoyarían la noción de un estado hiperactivo en esta red que podría interferir con la modulación de la actividad cerebral relacionada con la tarea y con el rendimiento cognitivo. Aunque debe tenerse en cuenta que en el estudio realizado los datos tanto de la ventana de preestímulo como de respuesta se obtuvieron durante la realización de una tarea, mientras que las evaluaciones funcionales de la DMN citadas se han realizado en estado de reposo.

Finalmente, de acuerdo a los resultados, la duración de la enfermedad y el tratamiento con antipsicóticos y otros grupos de psicofármacos examinados no explicarían las alteraciones encontradas en la CS. 


\subsection{Potencia de ruido cortical en la banda y ( $\mathrm{Y}-\mathrm{NP})$}

Los resultados muestran valores de la NP- $\gamma$ más altos en las regiones de la línea media frontal y parietal en la esquizofrenia en comparación con los controles. Este hallazgo concuerda y podría ser explicado con la evidencia de una sincronización neural alterada (147) y de un desequilibrio de la actividad neuronal excitadora/inhibidora (E/I) que han sido descritos en este trastorno (264). También es consistente con un estudio anterior de la NP- $\gamma$, que encontró valores más altos en la región de la línea media en una muestra de pacientes distinta $(172,179)$, y con otros informes de valores elevados de la NP en regiones frontales en la esquizofrenia (176).

La distribución regional de estos valores alterados de la NP- $\gamma$ en la esquizofrenia también corresponde de manera aproximada con la DMN $(265,266)$, en la que se han descrito alteraciones en la desactivación durante la realización de una tarea cognitiva (261), relacionadas con anormalidades en la neurotransmisión gabaérgica (31). Si bien los orígenes neurofisiológicos de la NP- $\gamma$ no están definidos con exactitud (se relaciona con el aumento de la variabilidad de las respuestas y con el aumento de la actividad cortical no relacionada con el estímulo), estos resultados sugieren la posible existencia de un mecanismo de hiperactivación común que va en detrimento de la activación/ desactivación satisfactoria de las asambleas neuronales requerida durante una tarea cognitiva (174).

En el trastorno bipolar se observó una NP- $\gamma$ más alta en el área frontal izquierda en comparación con los controles. Este resultado puede indicar asimismo la posible presencia de un estado de hiperactivación neuronal en el trastorno bipolar similar al encontrado en la esquizofrenia, aunque más leve y con una distribución regional aparentemente distinta. Un estudio previo de la NP- $\gamma$ que comparó muestras diferentes de pacientes con trastorno bipolar y controles no encontró diferencias significativas entre estos dos grupos (177). Pero no se descarta que esta discrepancia podría estar relacionada con diferencias metodológicas, ya que los análisis de ese estudio previo se basaron en un registro de EEG de menor resolución (17 electrodos) y se utilizó un análisis factorial de los valores de la NP- $\gamma(177)$, mientras que esta investigación se realizó 
con registros con un mayor número de electrodos y se siguió un análisis estadístico diferente, utilizando comparaciones corregidas por Bonferroni de los valores de la NP- $\gamma$ en cada uno de los electrodos.

Aunque esta investigación evalúa específicamente la actividad neuronal en la banda $\gamma$, los resultados de investigaciones previas de la NP en otras bandas de frecuencia han sido contradictorios. Un estudio informó valores elevados de la NP en todas las bandas de frecuencias en la esquizofrenia en comparación con controles sanos (176), mientras que otro no detectó diferencias significativas de la NP en la banda $\theta$ (179). Nuevamente, diferencias metodológicas podrían explicar estas diferencias, ya que, por definición las estimaciones de la NP señalan la variabilidad de las respuestas a un estímulo y por tanto son sensibles a los aspectos metodológicos que determinan estas respuestas.

No se encontró relación entre los valores alterados de la NP- $\gamma$ y la duración de la enfermedad o el tratamiento antipsicótico ni con los otros grupos de psicofármacos estudiados (benzodiacepinas, antidepresivos o estabilizadores del ánimo). Esto sugiere que las alteraciones encontradas en los pacientes son independientes de la duración de la enfermedad y del tratamiento farmacológico.

Los pacientes mostraron un peor rendimiento cognitivo comparados con los controles en la mayoría de las funciones de la cognición estudiadas. Sin embargo, no se encontró una relación significativa entre los valores alterados de la NP- $\gamma$ y el factor cognitivo general, que incluía todos los puntajes BACS, excepto la torre de Londres. Aunque una investigación de la NP- $\gamma$ en la esquizofrenia que correlacionó los valores de NP con los dominios individuales de la cognición informó de una correlación con un peor desempeño en la memoria de trabajo y resolución de problemas $(179,224)$. No obstante, debe tenerse en cuenta que la NP- $\gamma$ fue calculada durante un paradigma oddball (una tarea de atención y memoria de trabajo) pero no durante la propia realización de la batería cognitiva BACS, durante la cual es probable que se ejecuten procesos neuronales más complejos (267). Por lo tanto, no se descarta de manera definitiva una relación relevante entre la cognición y la NP- $\gamma$ que no ha sido detectada en el presente estudio.

Se observó una relación directa entre los valores elevados de la NP- $\gamma$ en las regiones frontal izquierda y parietotemporal y síntomas negativos en la esquizofrenia. Estos hallazgos corresponden 
aproximadamente a la correlación directa entre una mayor NP- $\gamma$ y puntuaciones PANSS negativas informadas en publicaciones previas en pacientes con esquizofrenia en el primer episodio (224). Esta relación con los síntomas negativos podría sugerir que el estado de hiperactividad neuronal ineficaz en la banda $\gamma$ indicado por la NP, probablemente interfiera con los mecanismos neuronales que sustentan los procesos afectivos. Por otro lado, aunque en el trastorno bipolar no se encontró una relación entre las alteraciones de la NP- $\gamma$ y las puntuaciones PANSS y de cognición, algunos análisis de la actividad EEG han mostrado alteraciones en las regiones frontales izquierdas relacionadas con características clínicas y cognitivas de este trastorno (267-270).

\subsection{Limitaciones}

La edad de los pacientes con trastorno bipolar fue mayor que la de los pacientes con esquizofrenia crónica, como probable consecuencia del diagnóstico más tardío del trastorno bipolar, aunque en todos los casos se intentó recolectar muestras con una duración de enfermedad similar.

La muestra de trastorno bipolar no incluyó pacientes en primeros episodios que permitiera hacer comparaciones entre estadios iniciales de la enfermedad y los crónicos.

Los tamaños de las muestras fueron relativamente pequeños, aunque estos son similares al de muchos otros estudios en este campo.

No se obtuvo una muestra de pacientes libres del todo de tratamiento farmacológico, que sería útil para ahondar en el posible papel del tratamiento en las alteraciones de la conectividad de la red. Algunos pacientes estaban recibiendo diferentes medicamentos al mismo tiempo y su interacción potencial no fue controlada.

En el estudio de conectividad estructural, no fueron consideradas las conexiones interhemisféricas para estimar los parámetros de la red.

El estudio de la actividad oscilatoria $\gamma$ se hace con estimaciones de la NP, que por definición se calcula durante la ejecución de una tarea cognitiva, y no se usó una medida equivalente durante el estado de reposo para su comparación. Esto podría ser de interés, ya que también se han encontrado alteraciones de la potencia espectral en la banda $\gamma$ durante el estado de reposo $(144,271)$. 
El estudio de la NP- $\gamma$ incluye únicamente a pacientes crónicos y no fue posible realizar comparaciones con estadios iniciales de la enfermedad (sujetos clínicos de alto riesgo o pacientes con primer episodio).

\subsection{Perspectivas de futuro}

Además de la duración de la enfermedad o el uso de tratamiento farmacológico, otros factores pueden tener una influencia negativa en la conectividad cerebral o de la potencia de ruido en los pacientes psicóticos crónicos, como el consumo temprano de drogas o el aislamiento social. De modo que los efectos potenciales correspondientes a estas variables podrían evaluarse con diseños específicos.

Es posible que el patrón de las alteraciones de la conectividad funcional encontrado (en la esquizofrenia aumento de la CS preestímulo en la banda global y reducción de la modulación en la banda $\theta$, mientras que en el trastorno bipolar solo la reducción de la modulación de la CS en la banda $\theta$ ) se relacione con un sustrato para estas anormalidades diferente entre estos dos grupos, lo que podría investigarse más a fondo.

También en el estudio de la conectividad funcional sería de interés evaluar la CS de los pacientes durante el estado de reposo (diferente al de la ventana preestímulo durante el desempeño de la tarea) y realizar análisis de fuentes apropiados para determinar las regiones clave responsables de las diferencias generales.

Ya que en pacientes con trastorno bipolar no se puede descartar una dependencia del estado, más que del rasgo, de las alteraciones de la conectividad y de la NP- $\gamma$, podrían realizarse estos análisis en evaluaciones longitudinales de las etapas depresiva, eutímica y maníaca. 


\section{CAPÍTULO 6}

\section{Conclusiones}

onsiderando los objetivos establecidos y en base a los resultados

obtenidos, las conclusiones de esta tesis son las siguientes:

I. Se observaron alteraciones de la conectividad cerebral tanto estructural como funcional en la esquizofrenia y el trastorno bipolar. Algunas de estas alteraciones son similares en estos dos trastornos psicóticos, lo que sugiere la existencia de algunos mecanismos subyacentes comunes. Por otra parte, los resultados también muestran diferencias importantes entre estos síndromes, en general a expensas de mayores alteraciones conectivas en la esquizofrenia crónica, que sugieren la coexistencia de mecanismos propios y específicos para este trastorno.

II. En cuanto a la conectividad estructural de la red cerebral, se observan alteraciones en la esquizofrenia crónica y en el trastorno bipolar, pero no en la esquizofrenia en el primer episodio psicótico.

III. Las alteraciones de la conectividad estructural no parecen relacionadas con el tratamiento antipsicótico, ya que no se encontró correlación entre la dosis de antipsicótico y los parámetros de conectividad, y estos no difirieron entre los pacientes bipolares que recibieron tratamiento antipsicótico y los que no lo recibieron.

IV. En la esquizofrenia crónica y en el trastorno bipolar hubo una relación directa entre los valores de conectividad estructural y el 
rendimiento en la memoria de trabajo y las pruebas de resolución de problemas.

V. En el estudio de la conectividad funcional de la red cerebral, el aumento del valor de la CS preestímulo en la banda global fue significativamente mayor en la esquizofrenia en comparación con el trastorno bipolar y con los controles sanos; que se asoció con déficits cognitivos y síntomas negativos. Este hallazgo puede indicar una sincronización hiperactiva entre grupos neuronales, específico de la esquizofrenia, que puede dificultar la cognición en este síndrome.

VI. La modulación de la CS relacionada con una tarea cognitiva en la banda $\theta$ fue menor en la esquizofrenia y el trastorno bipolar en comparación con controles sanos.

VII. Las alteraciones de la conectividad funcional no parecen relacionadas con el tratamiento antipsicótico ni la duración de la enfermedad. El tratamiento con antipsicóticos, litio, benzodiacepinas y anticonvulsivos tampoco parece influir en los valores de CS de la red funcional, y los pacientes con esquizofrenia en el primer episodio y los pacientes con esquizofrenia crónica no mostraron diferencias significativas en estos valores de CS.

VIII. En el estudio de la potencia del ruido cortical en la banda $\gamma$ se observó un aumento de los valores de la $\gamma$-NP anormalmente más altos en electrodos de la línea media (Fz y Pz) en la esquizofrenia, y en la región frontal izquierda (F7) en el trastorno bipolar.

IX. Estos resultados no parecen relacionados con el uso del tratamiento farmacológico ni con la duración de la enfermedad.

X. La distribución regional de las alteraciones encontradas en los estudios de conectividad funcional y de la potencia de ruido cortical en la banda $\gamma$, se corresponde en parte con la de la red neuronal por defecto, por lo que es plausible que un déficit en el mecanismo de inhibición neural pueda ser la base de la hipersincronización/ hiperactividad cortical ineficaz encontrada. 


\section{CAPÍTULO 7}

\section{Referencias}

1. Sporns O, Chialvo DR, Kaiser M, Hilgetag CC. Organization, development and function of complex brain networks. Trends Cogn Sci. 8(9):418-25.

http://linkinghub.elsevier.com/retrieve/pii/S1364661304001901

2. van den Heuvel MP, Mandl RCW, Stam CJ, Kahn RS, Hulshoff Pol HE. Aberrant frontal and temporal complex network structure in schizophrenia: a graph theoretical analysis. J Neurosci. 30(47):15915-26. http://www.jneurosci.org/cgi/doi/10.1523/JNEUROSCI.2874-10.2010

3. Wang Q, Su T-P, Zhou Y, Chou K-H, Chen I-Y, Jiang T, et al. Anatomical insights into disrupted small-world networks in schizophrenia. Neuroimage. 59(2):1085-93. https://linkinghub.elsevier.com/retrieve/pii/S1053811911010834

4. O’Neill A, Mechelli A, Bhattacharyya S. Dysconnectivity of large-scale functional networks in early psychosis: a meta-analysis. Schizophr Bull. 45(3):579-90.

https://academic.oup.com/schizophreniabulletin/article/45/3/579/5048697

5. van den Heuvel MP, Fornito A. Brain networks in schizophrenia. Neuropsychol Rev. 24(1):32-48. http://link.springer.com/10.1007/s11065014-9248-7

6. O’Donoghue S, Holleran L, Cannon DM, McDonald C. Anatomical dysconnectivity in bipolar disorder compared with schizophrenia: a selective review of structural network analyses using diffusion MRI. $J$ Affect Disord. 209:217-28.

https://linkinghub.elsevier.com/retrieve/pii/S0165032716310175

7. Perry A, Roberts G, Mitchell PB, Breakspear M. Connectomics of bipolar disorder: a critical review, and evidence for dynamic instabilities within interoceptive networks. Mol Psychiatry. 24(9):1296-318. http:/www.nature.com/articles/s41380-018-0267-2

8. Zhang R, Shao R, Xu G, Lu W, Zheng W, Miao Q, et al. Aberrant brain 
structural-functional connectivity coupling in euthymic bipolar disorder. Hum Brain Mapp. 40(12):hbm.24608. https://onlinelibrary.wiley.com/doi/abs/10.1002/hbm.24608

9. Lois G, Linke J, Wessa M. Altered functional connectivity between emotional and cognitive resting state networks in euthymic bipolar I disorder patients. PLoS One. 9(10):e107829. https://onlinelibrary.wiley.com/doi/abs/10.1002/hbm.24608

10. Park H-J, Friston K. Structural and functional brain networks: from connections to cognition. Science (80- ). 342(6158):1238411-1238411. https://www.sciencemag.org/lookup/doi/10.1126/science.1238411

11. Fries P. A mechanism for cognitive dynamics: neuronal communication through neuronal coherence. Trends Cogn Sci. 9(10):474-80. https://inkinghub.elsevier.com/retrieve/pii/S1364661305002421

12. Womelsdorf T, Schoffelen J-M, Oostenveld R, Singer W, Desimone R, Engel AK, et al. Modulation of neuronal interactions through neuronal synchronization. Science (80- ). 316(5831):1609-12.

https://www.sciencemag.org/lookup/doi/10.1126/science.1139597

13. Lee K-H, Williams LM, Breakspear M, Gordon E. Synchronous gamma activity: a review and contribution to an integrative neuroscience model of schizophrenia. Brain Res Rev. 41(1):57-78.

https://www.sciencedirect.com/science/article/pii/S0165017302002205?vi a\%3Dihub

14. Başar E, Güntekin B, Atagün İ, Turp Gölbaşı B, Tülay E, Özerdem A. Brain's alpha activity is highly reduced in euthymic bipolar disorder patients. Cogn Neurodyn. 6(1):11-20. http://link.springer.com/10.1007/s11571-011-9172-y

15. Artieda J, Alegre M, Valencia M, Urrestarazu E, Pérez Alcázar M, Nicolás MJ, et al. Brain oscillations: pathophysiological and potentially therapeutic role in some neurological and psychiatric diseases. An Sist Sanit Navar. 32(Supl 3):45-60. https://recyt.fecyt.es/index.php/ASSN/article/view/8336/6485

16. Başar E. Brain oscillations in neuropsychiatric disease. Dialogues Clin Neurosci. 15(3):291-300. http://www.ncbi.nlm.nih.gov/pubmed/24174901

17. Allen P, Sommer IE, Jardri R, Eysenck MW, Hugdahl K. Extrinsic and default mode networks in psychiatric conditions: relationship to excitatory-inhibitory transmitter balance and early trauma. Neurosci Biobehav Rev. 99:90-100. https://linkinghub.elsevier.com/retrieve/pii/S0149763418307231

18. Gomez-Pilar J, Lubeiro A, Poza J, Hornero R, Ayuso M, Valcárcel C, et al. Functional EEG network analysis in schizophrenia: evidence of larger segregation and deficit of modulation. Prog NeuroPsychopharmacology Biol Psychiatry. 76:116-23. https://linkinghub.elsevier.com/retrieve/pii/S027858461630286X

19. Rubinov M, Knock SA, Stam CJ, Micheloyannis S, Harris AWF, 
Williams LM, et al. Small-world properties of nonlinear brain activity in schizophrenia. Hum Brain Mapp. 30(2):403-16.

http://doi.wiley.com/10.1002/hbm.20517

20. van den Heuvel MP, Stam CJ, Boersma M, Hulshoff Pol HE. Small-world and scale-free organization of voxel-based resting-state functional connectivity in the human brain. Neuroimage. 43(3):528-39. https://linkinghub.elsevier.com/retrieve/pii/S1053811908009130

21. Bassett DS, Bullmore E, Verchinski BA, Mattay VS, Weinberger DR, Meyer-Lindenberg A. Hierarchical organization of human cortical networks in health and schizophrenia. J Neurosci. 28(37):9239-48. http://www.jneurosci.org/cgi/doi/10.1523/JNEUROSCI.1929-08.2008

22. Buzsaki G, Draguhn A. Neuronal oscillations in cortical networks. Science (80- ). 304(5679):1926-9. http://www.sciencemag.org/cgi/doi/10.1126/science.1099745

23. Uhlhaas PJ, Singer W. Abnormal neural oscillations and synchrony in schizophrenia. Nat Rev Neurosci. 11(2):100-13. http://www.nature.com/articles/nrn2774

24. Uhlhaas PJ, Singer W. Neural synchrony in brain disorders: Relevance for cognitive dysfunctions and pathophysiology. Neuron. 52(1):155-68. https://linkinghub.elsevier.com/retrieve/pii/S0896627306007276

25. Uhlhaas PJ, Roux F, Rodriguez E, Rotarska-Jagiela A, Singer W. Neural synchrony and the development of cortical networks. Trends Cogn Sci. 14(2):72-80. https://linkinghub.elsevier.com/retrieve/pii/S1364661309002824

26. Gray CM, Singer W. Stimulus-specific neuronal oscillations in orientation columns of cat visual cortex. Proc Natl Acad Sci. 86(5):1698-702. http://www.pnas.org/cgi/doi/10.1073/pnas.86.5.1698

27. Buzsáki G. Rhythms of the brain. New York: Oxford University Press; $448 \mathrm{p}$.

28. Foss-Feig JH, Adkinson BD, Ji JL, Yang G, Srihari VH, McPartland JC, et al. Searching for cross-diagnostic convergence: neural mechanisms governing excitation and inhibition balance in schizophrenia and autism spectrum disorders. Biol Psychiatry. 81(10):848-61.

https://linkinghub.elsevier.com/retrieve/pii/S0006322317313604

29. Krystal JH, Anticevic A, Yang GJ, Dragoi G, Driesen NR, Wang X-J, et al. Impaired tuning of neural ensembles and the pathophysiology of schizophrenia: a translational and computational neuroscience perspective. Biol Psychiatry. 81(10):874-85. https://linkinghub.elsevier.com/retrieve/pii/S0006322317300367

30. Fee C, Banasr M, Sibille E. Somatostatin-positive gamma-aminobutyric acid interneuron deficits in depression: cortical microcircuit and therapeutic perspectives. Biol Psychiatry. 82(8):549-59. https://linkinghub.elsevier.com/retrieve/pii/S000632231731630X

31. Gonzalez-Burgos G, Fish KN, Lewis DA. GABA neuron alterations, 
cortical circuit dysfunction and cognitive deficits in schizophrenia. Neural Plast. 2011:1-24. http://www.hindawi.com/journals/np/2011/723184/

32. Hirvonen J, Wibral M, Palva JM, Singer W, Uhlhaas P, Palva S. Wholebrain source-reconstructed MEG-data reveal reduced long-range synchronization in chronic schizophrenia. eneuro. 4(5):ENEURO.033817.2017. http://eneuro.org/lookup/doi/10.1523/ENEURO.0338-17.2017

33. Steullet P, Cabungcal J-H, Coyle J, Didriksen M, Gill K, Grace AA, et al. Oxidative stress-driven parvalbumin interneuron impairment as a common mechanism in models of schizophrenia. Mol Psychiatry. 22(7):936-43. http://www.nature.com/articles/mp201747

34. Real Academia Nacional de Medicina. Diccionario de términos médicos. 1 ed. Editorial Médica Panamericana; 1800 p.

35. Lieberman JA, First MB. Psychotic disorders. Ropper AH, editor. $N$ Engl J Med. 379(3):270-80. http://www.nejm.org/doi/10.1056/NEJMra1801490

36. Sadock BJ, Sadock VA, Ruiz P. Kaplan and Sadock's Comprehensive Textbook of Psychiatry. 10th Ed. Wolters Kluwer; 4997 p.

37. Pape K, Tamouza R, Leboyer M, Zipp F. Immunoneuropsychiatry novel perspectives on brain disorders. Nat Rev Neurol. 15(6):317-28. http://dx.doi.org/10.1038/s41582-019-0174-4

38. Delaney S, Fallon B, Alaedini A, Yolken R, Indart A, Feng T, et al. Inflammatory biomarkers in psychosis and clinical high risk populations. Schizophr Res. 206:440-3. https://doi.org/10.1016/j.schres.2018.10.017

39. van den Heuvel MP, Scholtens LH, Kahn RS. Multiscale neuroscience of psychiatric disorders. Biol Psychiatry. 86(7):512-22. https://doi.org/10.1016/j.biopsych.2019.05.015

40. Stengel E. Concepts of schizophrenia. BMJ. 1(5028):1174-6. https:/www.bmj.com/lookup/doi/10.1136/bmj.1.5028.1174

41. Kahn RS, Sommer IE, Murray RM, Meyer-Lindenberg A, Weinberger DR, Cannon TD, et al. Schizophrenia. Nat Rev Dis Prim. 1(1):15067. http://www.nature.com/articles/nrdp201567

42. Mueser KT, McGurk SR. Schizophrenia. Lancet. 363(9426):2063-72. https://linkinghub.elsevier.com/retrieve/pii/S0140673604164581

43. Dietz AG, Goldman SA, Nedergaard M. Glial cells in schizophrenia: a unified hypothesis. The Lancet Psychiatry. 7(3):272-81. https://linkinghub.elsevier.com/retrieve/pii/S2215036619303025

44. Sadock BJ, Ahmad S, Sadock VA. Kaplan \& Sadock. Manual de bolsillo de psiquiatría clínica Kaplan \& Sadock. $6^{\mathrm{a}}$ Ed. Wolters Kluwer; 617 p.

45. American Psychiatric Association. Diagnostic and Statistical Manual of Mental Disorders. Fifth Edition, DSM-5. American Psychiatric Association; $5^{\text {th }}$ Revised Edition; $991 \mathrm{p}$.

46. Akiskal HS. La nueva era bipolar. En: Akiskal HS, Cetkovich-Bakmas MG, García-Bonetto G, Strejilevich SA, Vázquez GH, editores. Trastornos bipolares Conceptos clínicos, neurobiológicos y terapéuticos. 1 
Ed. Editorial Médica Panamericana; p. 354.

47. Saunders KEA, Goodwin GM. The course of bipolar disorder. $A d v$ Psychiatr Treat. 16(5):318-28.

https://www.cambridge.org/core/product/identifier/S135551460000691X/t ype/journal_article

48. Bobo $\mathrm{W}$ V. The diagnosis and management of bipolar I and II disorders: clinical practice update. Mayo Clin Proc. 92(10):1532-51. http://dx.doi.org/10.1016/j.mayocp.2017.06.022

49. Grande I, Berk M, Birmaher B, Vieta E. Bipolar disorder. Lancet. 387(10027):1561-72. http://dx.doi.org/10.1016/S0140-6736(15)00241-X

50. Grunze H. Bipolar Disorder. En: Zigmond MJ, Rowland LP, Coyle JT, editores. Neurobiology of Brain Disorders: Biological Basis of Neurological and Psychiatric Disorders. Academic Press; p. 655-73.

51. Azevedo FAC, Carvalho LRB, Grinberg LT, Farfel JM, Ferretti REL, Leite REP, et al. Equal numbers of neuronal and nonneuronal cells make the human brain an isometrically scaled-up primate brain. J Comp Neurol. 513(5):532-41. http://doi.wiley.com/10.1002/cne.21974

52. Kandel ER, Schwartz JH, Jessell TM, Siegelbaum SA, Hudspeth AJ. Principles of neural science. 5th ed. New York: McGraw-Hill Medical; 1709 p.

53. Pakkenberg B. Aging and the human neocortex. Exp Gerontol. 38(12):95-9. https://linkinghub.elsevier.com/retrieve/pii/S0531556502001511

54. Sporns O. Networks of the brain. MIT Press; 412 p.

55. Sporns O. The future of network neuroscience. Netw Neurosci. 1(1):1-2. https://www.mitpressjournals.org/doi/abs/10.1162/NETN_e_00005

56. Newman M. Networks: An introduction. OUP Oxford; 784 p.

57. Newman M, Barabási A-L, Watts DJ. The structure and dynamics of networks. Princeton University Press; 582 p.

58. Fornito A, Zalesky A, Bullmore ET. Fundamentals of brain network analysis. Fundamentals of Brain Network Analysis. Elsevier; 476 p.

59. Papo D, Buldú JM, Boccaletti S, Bullmore ET. Complex network theory and the brain. Philos Trans R Soc B Biol Sci.369(1653):20130520. https://royalsocietypublishing.org/doi/10.1098/rstb.2013.0520

60. Lin A, Liu KKL, Bartsch RP, Ivanov PC. Dynamic network interactions among distinct brain rhythms as a hallmark of physiologic state and function. Commun Biol. 3(1):197. http://www.nature.com/articles/s42003020-0878-4

61. Power JD, Cohen AL, Nelson SM, Wig GS, Barnes KA, Church JA, et al. Functional network organization of the human brain. Neuron. 72(4):66578. https://linkinghub.elsevier.com/retrieve/pii/S0896627311007926

62. Thomas Yeo BT, Krienen FM, Sepulcre J, Sabuncu MR, Lashkari D, Hollinshead M, et al. The organization of the human cerebral cortex estimated by intrinsic functional connectivity. J Neurophysiol. 
106(3):1125-65. https://www.physiology.org/doi/10.1152/jn.00338.2011

63. Kawakubo H, Matsui Y, Kushima I, Ozaki N, Shimamura T. A network of networks approach for modeling interconnected brain tissue-specific networks. Berger B, editor. Bioinformatics. 35(17):3092-101. https://academic.oup.com/bioinformatics/article/35/17/3092/5289537

64. Bassett DS, Bullmore ET. Human brain networks in health and disease. Curr Opin Neurol. 22(4):340-7. https://journals.lww.com/00019052200908000-00003

65. Bassett DS, Siebenhühner F. Multiscale Network Organization in the Human Brain. En: Pesenson MM, Schuster HG, editores. Multiscale Analysis and Nonlinear Dynamics: From Genes to the Brain. Wiley-VCH Verlag GmbH \& Co. KGaA; p. 179-204.

66. Petersen SE, Sporns O. Brain networks and cognitive architectures. Neuron. 88(1):207-19.

https://linkinghub.elsevier.com/retrieve/pii/S0896627315008168

67. Bassett DS, Sporns O. Network neuroscience. Nat Neurosci. 20(3):35364. http://www.nature.com/articles/nn.4502

68. Maestú Unturbe F, Pereda de Pablo E, del Pozo Guerrero F. Conectividad funcional y anatómica en el cerebro humano. Análisis de señales y aplicaciones en ciencias de la salud. Barcelona: Elsevier; 288 p.

69. Sporns O. Structure and function of complex brain networks. Dialogues Clin Neurosci. 15(3):247-62.

http://www.pubmedcentral.nih.gov/articlerender.fcgi?artid=PMC3811098

70. Friston KJ. Functional and effective connectivity in neuroimaging: a synthesis. Hum Brain Mapp. 2(1-2):56-78. http://doi.wiley.com/10.1002/hbm.460020107

71. Mišić B, McIntosh AR. Effective connectivity. En: Brain Mapping: An Encyclopedic Reference. Elsevier Inc.; p. 587-92.

72. Bullmore E, Sporns O. Complex brain networks: graph theoretical analysis of structural and functional systems. Nat Rev Neurosci. 10(3):186-98. http://www.nature.com/articles/nrn2575

73. Power JD, Fair DA, Schlaggar BL, Petersen SE. The development of human functional brain networks. Neuron. 67(5):735-48. https://linkinghub.elsevier.com/retrieve/pii/S0896627310006276

74. Stam CJ, van Straaten ECW. The organization of physiological brain networks. Clin Neurophysiol. 123(6):1067-87. https://linkinghub.elsevier.com/retrieve/pii/S1388245712000570

75. Stam CJ. Characterization of anatomical and functional connectivity in the brain: a complex networks perspective. Int J Psychophysiol. 77(3):186-94. https://linkinghub.elsevier.com/retrieve/pii/S0167876010001686

76. Newman MEJ. The structure and function of complex networks. SIAM Rev. 45(2):167-256.

http://epubs.siam.org/doi/10.1137/S003614450342480 
77. Boccaletti S, Latora V, Moreno Y, Chavez M, Hwang D. Complex networks: structure and dynamics. Phys Rep. 424(4-5):175-308. https://linkinghub.elsevier.com/retrieve/pii/S037015730500462X

78. Sejnowski TJ, Churchland PS, Movshon JA. Putting big data to good use in neuroscience. Nat Neurosci. 17(11):1440-1.

http://www.nature.com/articles/nn.3839

79. Betzel RF, Bassett DS. Multi-scale brain networks. Neuroimage. 160:7383. https://linkinghub.elsevier.com/retrieve/pii/S1053811916306152

80. Costa L da F, Rodrigues FA, Travieso G, Boas PRV. Characterization of complex networks: a survey of measurements. Adv Phys. 56(1):167-242. http://arxiv.org/abs/cond-mat/0505185

81. Strogatz SH. Exploring complex networks. Nature. 410(6825):268-76. http://www.nature.com/articles/35065725

82. Albert R, Barabási A-L. Statistical mechanics of complex networks. Rev Mod Phys. 74(1):47-97.

https://link.aps.org/doi/10.1103/RevModPhys.74.47

83. Watts DJ, Strogatz SH. Collective dynamics of 'small-world' networks. Nature. 393(6684):440-2. http://www.nature.com/articles/30918

84. Erdös P, Rényi A. On random graphs I. Publ Math. 6:290-7.

85. Barabási A-L, Albert R. Emergence of scaling in random networks. Science (80- ). 286(5439):509-12.

https://www.sciencemag.org/lookup/doi/10.1126/science.286.5439.509

86. Lynn CW, Bassett DS. The physics of brain network structure, function and control. Nat Rev Phys. 1(5):318-32. http://dx.doi.org/10.1038/s42254019-0040-8

87. Hilgetag CC, Goulas A. Is the brain really a small-world network? Brain Struct Funct. 221(4):2361-6. http://link.springer.com/10.1007/s00429015-1035-6

88. Papo D, Zanin M, Martínez JH, Buldú JM. Beware of the small-world neuroscientist! Front Hum Neurosci. 10:8-11.

http://journal.frontiersin.org/Article/10.3389/fnhum.2016.00096/abstract

89. Bassett DS, Bullmore E. Small-world brain networks. Neurosci.

12(6):512-23.

http://journals.sagepub.com/doi/10.1177/1073858406293182

90. Bassett DS, Bullmore ET. Small-world brain networks revisited.

Neurosci. 23(5):499-516.

http://journals.sagepub.com/doi/10.1177/1073858416667720

91. Yu S, Huang D, Singer W, Nikolić D. A small world of neuronal synchrony. Cereb Cortex. 18(12):2891-901.

https://academic.oup.com/cercor/articlelookup/doi/10.1093/cercor/bhn047

92. Bucolo M, Fazzino S, La Rosa M, Fortuna L. Small-world networks of fuzzy chaotic oscillators. Chaos, Solitons \& Fractals. 17(2-3):557-65. 
https://linkinghub.elsevier.com/retrieve/pii/S0960077902003983

93. Lago-Fernández LF, Huerta R, Corbacho F, Sigüenza JA. Fast response and temporal coherent oscillations in small-world networks. Phys Rev Lett. 84(12):2758-61. https://link.aps.org/doi/10.1103/PhysRevLett.84.2758

94. Stam CJ, Reijneveld JC. Graph theoretical analysis of complex networks in the brain. Nonlinear Biomed Phys. 1(1):3. https://nonlinearbiomedphys.biomedcentral.com/articles/10.1186/17534631-1-3

95. Achard S, Bullmore E. Efficiency and cost of economical brain functional networks. Friston KJ, editor. PLoS Comput Biol. 3(2):e17. https://dx.plos.org/10.1371/journal.pcbi.0030017

96. Hallquist MN, Hillary FG. Graph theory approaches to functional network organization in brain disorders: a critique for a brave new small-world. Netw Neurosci. 3(1):1-26. https://www.mitpressjournals.org/doi/abs/10.1162/netn_a_00054

97. Sporns O, Tononi G, Kötter R. The human connectome: a structural description of the human brain. PLoS Comput Biol. 1(4):e42. https://dx.plos.org/10.1371/journal.pcbi.0010042

98. Bullmore ET, Bassett DS. Brain graphs: graphical models of the human brain connectome. Annu Rev Clin Psychol. 7(1):113-40. http:/www.annualreviews.org/doi/10.1146/annurev-clinpsy-040510143934

99. Butts CT. Revisiting the foundations of network analysis. Science (80-). 325(5939):414-6.

https://www.sciencemag.org/lookup/doi/10.1126/science.1171022

100. Rubinov M, Sporns O. Complex network measures of brain connectivity: uses and interpretations. Neuroimage. 52(3):1059-69. https://linkinghub.elsevier.com/retrieve/pii/S105381190901074X

101. Sporns O. Graph theory methods: applications in brain networks. Dialogues Clin Neurosci. 20(2):111-20. https://pubmed.ncbi.nlm.nih.gov/30250388/

102. Farahani F V., Karwowski W, Lighthall NR. Application of graph theory for identifying connectivity patterns in human brain networks: a systematic review. Front Neurosci. 13:585. https://www.frontiersin.org/article/10.3389/fnins.2019.00585/full

103. Finotelli P, Dulio P. Graph theoretical analysis of the brain. An overview. Sci e Ric. (July):89-96.

104. Kaiser M. A tutorial in connectome analysis: topological and spatial features of brain networks. Neuroimage. 57(3):892-907. https://linkinghub.elsevier.com/retrieve/pii/S1053811911005131

105. Stam CJ, de Haan W, Daffertshofer A, Jones BF, Manshanden I, van Cappellen van Walsum AM, et al. Graph theoretical analysis of magnetoencephalographic functional connectivity in Alzheimer's disease. 
Brain. 132(1):213-24. https://academic.oup.com/brain/articlelookup/doi/10.1093/brain/awn262

106. Gomez-Pilar J, Poza J, Bachiller A, Gómez C, Núñez P, Lubeiro A, et al. Quantification of graph complexity based on the edge weight distribution balance: application to brain networks. Int J Neural Syst. 28(01):1750032. https://www.worldscientific.com/doi/abs/10.1142/S0129065717500320

107. Bassett DS, Zurn P, Gold JI. On the nature and use of models in network neuroscience. Nat Rev Neurosci. 19(9):566-78. http://dx.doi.org/10.1038/s41583-018-0038-8

108. Humphries M., Gurney K, Prescott T. The brainstem reticular formation is a small-world, not scale-free, network. Proc R Soc B Biol Sci. 273(1585):503-11. https://royalsocietypublishing.org/doi/10.1098/rspb.2005.3354

109. Gomez-Pilar J, de Luis-García R, Lubeiro A, de Uribe N, Poza J, Núñez $\mathrm{P}$, et al. Deficits of entropy modulation in schizophrenia are predicted by functional connectivity strength in the theta band and structural clustering. NeuroImage Clin. 18:382-9.

https://linkinghub.elsevier.com/retrieve/pii/S2213158218300391

110. Buzsáki G, Watson BO. Brain rhythms and neural syntax: implications for efficient coding of cognitive content and neuropsychiatric disease.

Dialogues Clin Neurosci. 14(4):345-67.

http://www.ncbi.nlm.nih.gov/pubmed/23393413

111. Ford JM, Krystal JH, Mathalon DH. Neural synchrony in schizophrenia: From networks to new treatments. Schizophr Bull. 33(4):848-52. https://academic.oup.com/schizophreniabulletin/articlelookup/doi/10.1093/schbul/sbm062

112. Lopes da Silva F. EEG and MEG: relevance to neuroscience. Neuron. 80(5):1112-28. https://linkinghub.elsevier.com/retrieve/pii/S0896627313009203

113. Neustadter E, Mathiak K, Turetsky BI. EEG and MEG Probes of Schizophrenia Pathophysiology. En: Abel T, Nickl-Jockschat T, editores. The Neurobiology of Schizophrenia. Academic Press; p. 213-36.

114. Roach BJ, Mathalon DH. Event-related EEG time-frequency analysis: an overview of measures and an analysis of early gamma band phase locking in schizophrenia. Schizophr Bull. 34(5):907-26.

https://academic.oup.com/schizophreniabulletin/articlelookup/doi/10.1093/schbul/sbn093

115. Fröhlich F. Network Neuroscience. 1st ed. Academic Press; 482 p.

116. Mathalon DH, Sohal VS. Neural oscillations and synchrony in brain dysfunction and neuropsychiatric disorders. JAMA Psychiatry. 72(8):840. http://archpsyc.jamanetwork.com/article.aspx?doi=10.1001/jamapsychiatr y.2015.0483

117. Poulet JFA, Petersen CCH. Internal brain state regulates membrane potential synchrony in barrel cortex of behaving mice. Nature. 
454(7206):881-5. http://www.nature.com/articles/nature07150

118. Buzsáki G, Anastassiou CA, Koch C. The origin of extracellular fields and currents - EEG, ECoG, LFP and spikes. Nat Rev Neurosci. 13(6):407-20. http://www.nature.com/articles/nrn3241

119. Sultan KT, Brown KN, Shi S-H. Production and organization of neocortical interneurons. Front Cell Neurosci. 7:221. http://journal.frontiersin.org/article/10.3389/fncel.2013.00221/abstract

120. Marcuse L, Fields M, Yoo J. Rowan's Prmier of EEG. 2nd ed. Elsevier; $216 \mathrm{p}$.

121. Stam CJ, Nolte G, Daffertshofer A. Phase lag index: assessment of functional connectivity from multi channel EEG and MEG with diminished bias from common sources. Hum Brain Mapp. 28(11):117893. http://doi.wiley.com/10.1002/hbm.20346

122. Rossini PM, Ferreri F. Neurophysiological techniques in the study of the excitability, connectivity, and plasticity of the human brain. En: Başar E, Başar-Eroglu C, Özerdem A, Rossini PM, Yener GG, editores.

Application of Brain Oscillations in Neuropsychiatric Diseases. 1. ${ }^{a}$ ed. Elsevier B.V.; p. 1-17.

123. He B, Astolfi L, Valdes-Sosa PA, Marinazzo D, Palva SO, Benar C-G, et al. Electrophysiological brain connectivity: theory and implementation. IEEE Trans Biomed Eng. 66(7):2115-37. https://ieeexplore.ieee.org/document/8708690/

124. Capilla A, Carretié L. Bases neurofisiológicas de las oscilaciones cerebrales. En: Maestú F, Pereda E, del Pozo F, editores. Conectividad funcional y anatómica en el cerebro humano. Análisis de señales y aplicaciones en ciencias de la salud. Elsevier; p. 29-37.

125. Schnitzler A, Gross J. Functional connectivity analysis in magnetoencephalography. En: International Review of Neurobiology. Int Rev Neurobiol; p. 173-95.

126. Steriade M, McCormick D, Sejnowski T. Thalamocortical oscillations in the sleeping and aroused brain. Science (80- ). 262(5134):679-85. https://www.sciencemag.org/lookup/doi/10.1126/science. 8235588

127. Penttonen M, Buzsáki G. Natural logarithmic relationship between brain oscillators. Thalamus Relat Syst. 2(02):145. http://www.journals.cambridge.org/abstract_S1472928803000074

128. Berger H. Über das Elektrenkephalogramm des Menschen. Arch Psychiatr Nervenkr. 87:527-70.

129. A glossary of terms most commonly used by clinical electroencephalographers. Electroencephalogr Clin Neurophysiol. 37(5):538-48. https://linkinghub.elsevier.com/retrieve/pii/0013469474900996

130. Babiloni C, Barry RJ, Başar E, Blinowska KJ, Cichocki A, Drinkenburg WHIM, et al. International Federation of Clinical Neurophysiology (IFCN) - EEG research workgroup: Recommendations on frequency and 
topographic analysis of resting state EEG rhythms. Part 1: Applications in clinical research studies. Clin Neurophysiol. 131(1):285-307. https://linkinghub.elsevier.com/retrieve/pii/S1388245719311642

131. Kane N, Acharya J, Beniczky S, Caboclo L, Finnigan S, Kaplan PW, et al. A revised glossary of terms most commonly used by clinical electroencephalographers and updated proposal for the report format of the EEG findings. Revision 2017. Clin Neurophysiol Pract. 2:170-85. https://linkinghub.elsevier.com/retrieve/pii/S2467981X17300215

132. Uhlhaas PJ, Haenschel C, Nikolić D, Singer W. The role of oscillations and synchrony in cortical networks and their putative relevance for the pathophysiology of schizophrenia. Schizophr Bull. 34(5):927-43. https://academic.oup.com/schizophreniabulletin/article/34/5/927/1881444

133. Buzsáki G. Theta rhythm of navigation: Link between path integration and landmark navigation, episodic and semantic memory. Hippocampus. 15(7):827-40. http://doi.wiley.com/10.1002/hipo.20113

134. Kahana MJ, Seelig D, Madsen JR. Theta returns. Curr Opin Neurobiol. 11(6):739-44.

https://linkinghub.elsevier.com/retrieve/pii/S0959438801002781

135. Herweg NA, Solomon EA, Kahana MJ. Theta oscillations in human memory. Trends Cogn Sci. 24(3):208-27.

https://linkinghub.elsevier.com/retrieve/pii/S1364661319302943

136. Hensch TK, Fagiolini M, editores. Excitatory-Inhibitory Balance Synapses, Circuits, Systems. Springer US; 288 p.

137. Tatti R, Haley MS, Swanson OK, Tselha T, Maffei A. Neurophysiology and regulation of the balance between excitation and inhibition in neocortical circuits. Biol Psychiatry. 81(10):821-31. http://dx.doi.org/10.1016/j.biopsych.2016.09.017

138. Ferguson BR, Gao W-J. PV interneurons: critical regulators of E/I balance for prefrontal cortex-dependent behavior and psychiatric disorders. Front Neural Circuits. 12(May):1-13. http://journal.frontiersin.org/article/10.3389/fncir.2018.00037/full

139. Sohal VS, Rubenstein JLR. Excitation-inhibition balance as a framework for investigating mechanisms in neuropsychiatric disorders. Mol Psychiatry. 24(9):1248-57. http://dx.doi.org/10.1038/s41380-019-0426-0

140. Gao R, Penzes P. Common mechanisms of excitatory and inhibitory imbalance in schizophrenia and autism spectrum disorders. Curr Mol Med. 15(2):146-67.

http://www.eurekaselect.com/openurl/content.php?genre=article\&issn=15 66-5240\&volume $=15$ \& issue $=2 \&$ spage $=146$

141. Rubenstein JLR, Merzenich MM. Model of autism: increased ratio of excitation/inhibition in key neural systems. Genes, Brain Behav. 2(5):25567. http://doi.wiley.com/10.1034/j.1601-183X.2003.00037.x

142. Tiesinga $P$, Sejnowski TJ. Cortical enlightenment: are attentional gamma oscillations driven by ING or PING? Neuron. 63(6):727-32. 
http://dx.doi.org/10.1016/j.neuron.2009.09.009

143. Cardin JA, Carlén M, Meletis K, Knoblich U, Zhang F, Deisseroth K, et al. Driving fast-spiking cells induces gamma rhythm and controls sensory responses. Nature. 459(7247):663-7. http://www.nature.com/articles/nature08002

144. Grent-’t-Jong T, Gross J, Goense J, Wibral M, Gajwani R, Gumley AI, et al. Resting-state gamma-band power alterations in schizophrenia reveal E/I-balance abnormalities across illness-stages. Elife. 7.

https://elifesciences.org/articles/37799

145. Uhlhaas PJ, Singer W. Neuronal dynamics and neuropsychiatric disorders: toward a translational paradigm for dysfunctional large-scale networks.

Neuron. 75(6):963-80.

https://linkinghub.elsevier.com/retrieve/pii/S0896627312008112

146. Gonzalez-Burgos G, Lewis DA. NMDA receptor hypofunction, parvalbumin-positive neurons, and cortical gamma oscillations in schizophrenia. Schizophr Bull. 38(5):950-7.

https://academic.oup.com/schizophreniabulletin/articlelookup/doi/10.1093/schbul/sbs010

147. Gandal MJ, Edgar JC, Klook K, Siegel SJ. Gamma synchrony: towards a translational biomarker for the treatment-resistant symptoms of schizophrenia. Neuropharmacology. 62(3):1504-18. https://inkinghub.elsevier.com/retrieve/pii/S0028390811000773

148. Spencer KM, Nestor PG, Niznikiewicz MA, Salisbury DF, Shenton ME, McCarley RW. Abnormal neural synchrony in schizophrenia. J Neurosci. 23(19):7407-11. http://www.jneurosci.org/lookup/doi/10.1523/JNEUROSCI.23-1907407.2003

149. Newson JJ, Thiagarajan TC. EEG frequency bands in psychiatric disorders: a review of resting state studies. Front Hum Neurosci. 12:521. https://www.frontiersin.org/article/10.3389/fnhum.2018.00521/full

150. Crick F, Koch C. Towards a neurobiological theory of consciousness. Semin Neurosci. 2:263-75.

151. Merker B. Cortical gamma oscillations: the functional key is activation, not cognition. Neurosci Biobehav Rev. 37(3):401-17.

http://dx.doi.org/10.1016/j.neubiorev.2013.01.013

152. Fries P, Neuenschwander S, Engel AK, Goebel R, Singer W. Rapid feature selective neuronal synchronization through correlated latency shifting. Nat Neurosci. 4(2):194-200. http://www.nature.com/articles/nn0201_194

153. Varela F, Lachaux J-P, Rodriguez E, Martinerie J. The brainweb: phase synchronization and large-scale integration. Nat Rev Neurosci. 2(4):22939. http://www.nature.com/articles/35067550

154. Başar E. A review of gamma oscillations in healthy subjects and in cognitive impairment. Int J Psychophysiol. 90(2):99-117. 
https://www.sciencedirect.com/science/article/pii/S0167876013002134

155. Palva JM, Palva S. Functional integration across oscillation frequencies by cross-frequency phase synchronization. Eur J Neurosci. 48(7):2399406. http://doi.wiley.com/10.1111/ejn.13767

156. Salinas E, Sejnowski TJ. Correlated neuronal activity and the flow of neural information. Nat Rev Neurosci. 2(8):539-50.

http://www.nature.com/articles/35086012

157. Klimesch W. The frequency architecture of brain and brain body oscillations: an analysis. Eur J Neurosci. 48(7):2431-53. https://onlinelibrary.wiley.com/doi/10.1111/ejn.14192

158. Gougelet RJ. Neural Oscillation Dynamics of Emerging Interest in Neuroergonomics. En: Ayaz H, Dehais F, editores. Neuroergonomics: The Brain at Work and in Everyday Life. Academic Press; p. 87-91.

159. Sauseng P, Klimesch W. What does phase information of oscillatory brain activity tell us about cognitive processes? Neurosci Biobehav Rev. 32(5):1001-13. https://linkinghub.elsevier.com/retrieve/pii/S0149763408000493

160. Gross J. Analytical methods and experimental approaches for electrophysiological studies of brain oscillations. J Neurosci Methods. 228:57-66. http://dx.doi.org/10.1016/j.jneumeth.2014.03.007

161. Niso G, Bajo R, Castellanos NP, Pereda E. Estudio de la conectividad funcional y efectiva en EEG/MEG. En: Maestú F, Pereda E, del Pozo Guerrero F, editores. Conectividad funcional y anatómica en el cerebro humano. Análisis de señales y aplicaciones en ciencias de la salud. Barcelona: Elsevier; p. 39-50.

162. Penny WD, Kiebel SJ, Kilner JM, Rugg MD. Event-related brain dynamics. Trends Neurosci. 25(8):387-9.

https://www.sciencedirect.com/science/article/pii/S0166223602022026?vi $\mathrm{a} \% 3$ Dihub

163. Andrew C, Pfurtscheller G. Event-related coherence as a tool for studying dynamic interaction of brain regions. Electroencephalogr Clin Neurophysiol. 98(2):144-8. https://linkinghub.elsevier.com/retrieve/pii/0013469495002286

164. Sauseng P, Klimesch W, Gruber WR, Hanslmayr S, Freunberger R, Doppelmayr M. Are event-related potential components generated by phase resetting of brain oscillations? a critical discussion. Neuroscience. 146(4):1435-44. https://linkinghub.elsevier.com/retrieve/pii/S0306452207003314

165. Pfurtscheller G, Lopes da Silva FH. Event-related EEG/MEG synchronization and desynchronization: basic principles. Clin Neurophysiol. 110(11):1842-57. https://linkinghub.elsevier.com/retrieve/pii/S1388245799001418

166. Pfurtscheller G, Lopes da Silva F. EEG Event-Related Desynchronization (ERD) and Event-Related Synchronization (ERS). En: Schomer DL, 
Lopes da Silva FH, editores. Niedermeyer's Electroencephalography Basic Principles, Clinical Applications, and Related Fields. 6th ed. Lippincott Williams \& Wilkins (LWW); p. 935-48.

167. Bastiaansen M, Mazaheri A, Jensen O. Beyond ERPs: Oscillatory Neuronal Dynamics. En: The Oxford Handbook of Event-Related Potential Components. Oxford University Press.

168. Kappenman ES, Luck SJ. ERP Components: The Ups and Downs of Brainwave Recordings. En: Luck SJ, Kappenman ES, editores. The Oxford Handbook of Event-Related Potential Components. Oxford University Press.

169. Reinhart RMG, Mathalon DH, Roach BJ, Ford JM. Relationships between pre-stimulus gamma power and subsequent $\mathrm{P} 300$ and reaction time breakdown in schizophrenia. Int J Psychophysiol. 79(1):16-24. https://www.sciencedirect.com/science/article/pii/S0167876010006884

170. Polich J. Updating P300: an integrative theory of P3a and P3b. Clin Neurophysiol. 118(10):2128-48. https://linkinghub.elsevier.com/retrieve/pii/S1388245707001897

171. Winterer G, Ziller M, Dorn H, Frick K, Mulert C, Dahhan N, et al. Cortical activation, signal-to-noise ratio and stochastic resonance during information processing in man. Clin Neurophysiol. 110(7):1193-203. https://linkinghub.elsevier.com/retrieve/pii/S1388245799000590

172. Díez Á, Suazo V, Casado P, Martín-Loeches M, Molina V. Spatial distribution and cognitive correlates of gamma noise power in schizophrenia. Psychol Med. 43(6):1175-85. https://www.cambridge.org/core/product/identifier/S0033291712002103/t ype/journal_article

173. Huang Z, Zhang J, Longtin A, Dumont G, Duncan NW, Pokorny J, et al. Is there a nonadditive interaction between spontaneous and evoked activity? Phase-dependence and its relation to the temporal structure of scale-free brain activity. Cereb Cortex. 27(2):1037-59.

http://cercor.oxfordjournals.org/cgi/doi/10.1093/cercor/bhv288

174. Winterer G, Ziller M, Dorn H, Frick K, Mulert C, Wuebben Y, et al. Schizophrenia: reduced signal-to-noise ratio and impaired phase-locking during information processing. Clin Neurophysiol. 111(5):837-49. https://www.sciencedirect.com/science/article/pii/S1388245799003223?vi $\mathrm{a} \% 3$ Dihub

175. Möcks J, Gasser T, Köhler W. Basic statistical parameters of event-related potentials. J Psychophysiol. 2(1):61-70.

176. Winterer G, Coppola R, Goldberg TE, Egan MF, Jones DW, Sanchez CE, et al. Prefrontal broadband noise, working memory, and genetic risk for schizophrenia. Am J Psychiatry. 161(3):490-500.

http://psychiatryonline.org/doi/abs/10.1176/appi.ajp.161.3.490

177. Suazo V, Lubeiro A, Jurado-Barba R, Moreno-Ortega M, Dompablo M, Morales-Muñoz I, et al. Elevated midline-parietal gamma band noise 
power in schizophrenia but not in bipolar patients. Eur Arch Psychiatry Clin Neurosci. 266(8):743-53. http://link.springer.com/10.1007/s00406016-0673-x

178. Molina V, Bachiller A, Suazo V, Lubeiro A, Poza J, Hornero R. Noise power associated with decreased task-induced variability of brain electrical activity in schizophrenia. Eur Arch Psychiatry Clin Neurosci. 266(1):55-61. http://link.springer.com/10.1007/s00406-014-0569-6

179. Díez Á, Suazo V, Casado P, Martín-Loeches M, Perea MV, Molina V. Frontal gamma noise power and cognitive domains in schizophrenia. Psychiatry Res Neuroimaging. 221(1):104-13. https://linkinghub.elsevier.com/retrieve/pii/S0925492713003041

180. Winterer G. Cortical microcircuits in schizophrenia - The dopamine hypothesis revisited. Pharmacopsychiatry. 39(SUPPL. 1):68-71. http://www.thieme-connect.de/DOI/DOI?10.1055/s-2006-931498

181. Mori S, Zhang J. Principles of diffusion tensor imaging and its applications to basic neuroscience research. Neuron. 51(5):527-39. https:/linkinghub.elsevier.com/retrieve/pii/S0896627306006349

182. Jones DK. Studying connections in the living human brain with diffusion MRI. Cortex. 44(8):936-52.

https://linkinghub.elsevier.com/retrieve/pii/S001094520800110X

183. Tecchio F, Porcaro C, Barbati G, Zappasodi F. Functional source separation and hand cortical representation for a brain-computer interface feature extraction. $J$ Physiol. 580(3):703-21.

http://doi.wiley.com/10.1113/jphysiol.2007.129163

184. Mori S, Wanaka S, Nagae-Poetscher LM, van Zijl PCM. MRI Atlas of Human White Matter. 1st ed. Elsevier; 276 p.

185. Cochard LR, Netter FH. Netter's introduction to imaging. 1st ed. Elsevier; $281 \mathrm{p}$.

186. Ramón Botella E, Aguado del Hoyo A, Lafuente Martínez J. Imagen por resonancia magnética. Fundamentos técnicos. Riesgos. En: del Cura Rodríguez JL, Pedraza Gutiérrez S, Gayete Cara À, Rovira Cañellas À, editores. Radiología esencial. $2^{\mathrm{a}}$ Edición. Editorial Médica Panamericana; p. 2416.

187. Mori S. Introduction to Diffusion Tensor Imaging. 1st ed. Introduction to Diffusion Tensor Imaging. Elsevier Science; 176 p.

188. Basser PJ, Mattiello J, LeBihan D. MR diffusion tensor spectroscopy and imaging. Biophys J. 66(1):259-67.

https://linkinghub.elsevier.com/retrieve/pii/S0006349594807751

189. Le Bihan D, Breton E. Imagerie de diffusion in-vivo par résonance magnétique nucléaire. Comptes-Rendus l'Académie des Sci. 95(5):27-34.

190. Le Bihan D, Mangin J-F, Poupon C, Clark CA, Pappata S, Molko N, et al. Diffusion tensor imaging: concepts and applications. J Magn Reson Imaging. 13(4):534-46. http://doi.wiley.com/10.1002/jmri.1076 
191. Ellingson BM, Cohen-Adad J. Diffusion-Weighted Imaging of the Spinal Cord. En: Quantitative MRI of the Spinal Cord. Elsevier Inc.; p. 123-45.

192. Linden D. Neuroimaging and Neurophysiology in Psychiatry. Oxford University Press; 160 p.

193. Duque A, Roa E, Castedo J. Anatomía de la sustancia blanca mediante tractografía por tensor de difusión. Radiologia. 50(2):99-111. https://linkinghub.elsevier.com/retrieve/pii/S0033833808719441

194. Basser PJ, Jones DK. Diffusion-tensor MRI: theory, experimental design and data analysis - a technical review. NMR Biomed. 15(7-8):456-67. http://doi.wiley.com/10.1002/nbm.783

195. Ito R, Mori S, Melhem ER. Diffusion tensor brain imaging and tractography. Neuroimaging Clin N Am. 12(1):1-19. https://linkinghub.elsevier.com/retrieve/pii/S1052514903000674

196. Recio Rodríguez M, Hernández Aceituno D, Hernández González LC, Martínez de Vega Fernández V, Carrascoso J. Imagen de tractografía 3T: anatomía y aplicaciones clínicas. Radiologia. 55(1):57-68. https://linkinghub.elsevier.com/retrieve/pii/S0033833811003614

197. Mukherjee P, Berman JI, Chung SW, Hess CP, Henry RG. Diffusion tensor MR imaging and fiber tractography: theoretic underpinnings. Am J Neuroradiol. 29(4):632-41.

http://www.ajnr.org/lookup/doi/10.3174/ajnr.A1051

198. Basser PJ, Pierpaoli C. Microstructural and physiological features of tissues elucidated by quantitative-diffusion-tensor MRI. J Magn Reson. 213(2):560-70.

https://linkinghub.elsevier.com/retrieve/pii/S109078071100334X

199. Niedermeyer E, Schomer DL. Historical Aspects of EEG. En: Schomer DL, Lopes da Silva FH, editores. Niedermeyer's Electroencephalography Basic Principles, Clinical Applications, and Related Fields. 6th Ed. Lippincott Williams \& Wilkins (LWW); p. 1296.

200. Schomer DL, Lopes da Silva FH, editores. Niedermeyer's Electroencephalography: Basic Principles, Clinical Applications, and Related Fields. 6th Ed. Lippincott Williams \& Wilkins (LWW); 1296 p.

201. Stone JL, Hughes JR. Early history of electroencephalography and establishment of the american clinical neurophysiology society. $J$ Clin Neurophysiol. 30(1):28-44. http://journals.lww.com/00004691201302000-00002

202. Winterer G, Mccarley RW. Electrophysiology of schizophrenia. En: Weinberger DR, Harrison P, editores. Schizophrenia. 3rd ed. WileyBlackwell; p. 311-33.

203. Mikulan E, Russo S, Parmigiani S, Sarasso S, Zauli FM, Rubino A, et al. Simultaneous human intracerebral stimulation and HD-EEG, ground-truth for source localization methods. Sci Data. 7(1):127. http:/www.nature.com/articles/s41597-020-0467-x

204. Nunez PL, Srinivasan R. Electric Fields of the Brain: The neurophysics of 
EEG. 2nd ed. Oxford University Press; 640 p.

205. Siuly S, Li Y, Zhang Y. EEG Signal Analysis and Classification.

Techniques and Applications. 1st Ed. Springer International Publishing; $256 \mathrm{p}$.

206. Smith SW. The Scientist and Engineer's Guide to Digital Signal Processing. Second Edi. San Diego, CA: California Technical Publishing;

207. Nadin D. Generation of EEG signals [Internet]. https://app.biorender.com/community/gallery/s5ee0198d3f3e6700aace31cc-generation-of-eeg-signals

208. Nuwer MR, Comi G, Emerson R, Fuglsang-Frederiksen A, Guérit J-M, Hinrichs $\mathrm{H}$, et al. IFCN standards for digital recording of clinical EEG. Electroencephalogr Clin Neurophysiol. 106(3):259-61. https://linkinghub.elsevier.com/retrieve/pii/S0013469497001065

209. Wheeler AL, Wessa M, Szeszko PR, Foussias G, Chakravarty MM, Lerch JP, et al. Further neuroimaging evidence for the deficit subtype of schizophrenia. JAMA Psychiatry. 72(5):446.

http://archpsyc.jamanetwork.com/article.aspx?doi=10.1001/jamapsychiatr y.2014.3020

210. Zhang R, Wei Q, Kang Z, Zalesky A, Li M, Xu Y, et al. Disrupted brain anatomical connectivity in medication-naïve patients with first-episode schizophrenia. Brain Struct Funct. 220(2):1145-59.

http://link.springer.com/10.1007/s00429-014-0706-z

211. Skåtun KC, Kaufmann T, Tønnesen S, Biele G, Melle I, Agartz I, et al. Global brain connectivity alterations in patients with schizophrenia and bipolar spectrum disorders. J Psychiatry Neurosci. 41(5):331-41. http://jpn.ca/vol41-issue5/41-5-331/

212. Ji E, Guevara P, Guevara M, Grigis A, Labra N, Sarrazin S, et al. Increased and decreased superficial white matter structural connectivity in schizophrenia and bipolar disorder. Schizophr Bull. 45(6):1367-78. https://academic.oup.com/schizophreniabulletin/article/45/6/1367/543012 3

213. Leow A, Ajilore O, Zhan L, Arienzo D, GadElkarim J, Zhang A, et al. Impaired inter-hemispheric integration in bipolar disorder revealed with brain network analyses. Biol Psychiatry. 73(2):183-93.

https://linkinghub.elsevier.com/retrieve/pii/S0006322312008098

214. Li F, Lui S, Yao L, Ji G-J, Liao W, Sweeney JA, et al. Altered white matter connectivity within and between networks in antipsychotic-naive first-episode schizophrenia. Schizophr Bull. 44(2):409-18. https://academic.oup.com/schizophreniabulletin/article/44/2/409/3829524

215. Xiao Y, Sun H, Shi S, Jiang D, Tao B, Zhao Y, et al. White matter abnormalities in never-treated patients with long-term schizophrenia. Am J Psychiatry. 175(11):1129-36. http://ajp.psychiatryonline.org/doi/10.1176/appi.ajp.2018.17121402

216. Gomez-Pilar J, de Luis-García R, Lubeiro A, de la Red H, Poza J, Núñez 
P, et al. Relations between structural and EEG-based graph metrics in healthy controls and schizophrenia patients. Hum Brain Mapp.

39(8):3152-65. http://doi.wiley.com/10.1002/hbm.24066

217. Bachiller A, Díez A, Suazo V, Domínguez C, Ayuso M, Hornero R, et al. Decreased spectral entropy modulation in patients with schizophrenia during a P300 task. Eur Arch Psychiatry Clin Neurosci. 264(6):533-43. http://link.springer.com/10.1007/s00406-014-0488-6

218. Molina V, Bachiller A, Gomez-Pilar J, Lubeiro A, Hornero R, Cea-Cañas B, et al. Deficit of entropy modulation of the EEG in schizophrenia associated to cognitive performance and symptoms. A replication study. Schizophr Res. 195:334-42. https://linkinghub.elsevier.com/retrieve/pii/S0920996417305339

219. Yao L, Li F, Liu J, Liao W, Li X, Li M, et al. Functional brain networks in never-treated and treated long-term Ill schizophrenia patients.

Neuropsychopharmacology. 44(11):1940-7.

http://dx.doi.org/10.1038/s41386-019-0428-2

220. Wang LX, Guo F, Zhu YQ, Wang HN, Liu WM, Li C, et al. Effect of second-generation antipsychotics on brain network topology in firstepisode schizophrenia: a longitudinal rs-fMRI study. Schizophr Res. 208:160-6. https://doi.org/10.1016/j.schres.2019.03.015

221. Basar-Eroglu C, Brand A, Hildebrandt H, Karolina Kedzior K, Mathes B, Schmiedt C. Working memory related gamma oscillations in schizophrenia patients. Int J Psychophysiol. 64(1):39-45. https://www.sciencedirect.com/science/article/pii/S0167876006002133

222. Selten M, van Bokhoven H, Nadif Kasri N. Inhibitory control of the excitatory/inhibitory balance in psychiatric disorders. F1000Research. 7(May):23. https://f1000research.com/articles/7-23/v1

223. Murray JD, Wang X-J. Cortical circuit models in psychiatry: Linking disrupted excitation-inhibition balance to cognitive deficits associated with schizophrenia. En: Anticevic A, Murray JD, editores. Computational Psychiatry Mathematical Modeling of Mental Illness. Oxford: Academic Press; p. 3-25.

224. Suazo V, Díez Á, Martín C, Ballesteros A, Casado P, Martín-Loeches M, et al. Elevated noise power in gamma band related to negative symptoms and memory deficit in schizophrenia. Prog Neuro-Psychopharmacology Biol Psychiatry. 38(2):270-5.

https://linkinghub.elsevier.com/retrieve/pii/S0278584612000826

225. Wechsler D. Wechsler Adult Intelligence Scale-Third Edition. The Psychological Corporation. San Antonio, TX;

226. Grant DA, Berg E. A behavioral analysis of degree of reinforcement and ease of shifting to new responses in a Weigl-type card-sorting problem. $J$ Exp Psychol. 38(4):404-11.

227. Segarra N, Bernardo M, Gutierrez F, Justicia A, Fernadez-Egea E, Allas $\mathrm{M}$, et al. Spanish validation of the Brief Assessment in Cognition in 
Schizophrenia (BACS) in patients with schizophrenia and healthy controls. Eur Psychiatry. 26(2):69-73. http://linkinghub.elsevier.com/retrieve/pii/S092493380900193X

228. Mayer JD, Salovey P, Caruso DR, Sitarenios G. Measuring emotional intelligence with the MSCEIT V2.0. Emotion. 3(1):97-105. http://doi.apa.org/getdoi.cfm?doi=10.1037/1528-3542.3.1.97

229. Kay SR, Fiszbein A, Opler LA. The Positive and Negative Syndrome Scale (PANSS) for schizophrenia. Schizophr Bull. 13(2):261-76. https://academic.oup.com/schizophreniabulletin/articlelookup/doi/10.1093/schbul/13.2.261

230. Mané A, García-Rizo C, Garcia-Portilla MP, Bergé D, Sugranyes G, Garcia-Alvarez L, et al. Spanish adaptation and validation of the Brief Negative Symptoms Scale. Compr Psychiatry. 55(7):1726-9. https://linkinghub.elsevier.com/retrieve/pii/S0010440X14001503

231. Ramos-Brieva JA, Cordero Villafáfila A. Validación de la versión castellana de la escala de Hamilton para la depresión. Actas Luso Esp Neurol Psiquiatr Cienc Afines. 14(4):324-34.

http://www.ncbi.nlm.nih.gov/pubmed/3776732

232. Colom F, Vieta E, Martínez Arán A, García García M, Reinares M, Torrent $\mathrm{C}$, et al. Versión española de una escala de evaluación de la manía: validez y fiabilidad de la Escala de Young. Med Clin (Barc). 119(10):366-71. https://medes.com/publication/6650

233. Molina V, Lubeiro A, Soto O, Rodriguez M, Álvarez A, Hernández R, et al. Alterations in prefrontal connectivity in schizophrenia assessed using diffusion magnetic resonance imaging. Prog NeuroPsychopharmacology Biol Psychiatry. 76:107-15. https://linkinghub.elsevier.com/retrieve/pii/S0278584616303724

234. Tournier J-D, Smith R, Raffelt D, Tabbara R, Dhollander T, Pietsch M, et al. MRtrix3: a fast, flexible and open software framework for medical image processing and visualisation. Neuroimage. 202:116137. https://linkinghub.elsevier.com/retrieve/pii/S1053811919307281

235. Jenkinson M, Beckmann CF, Behrens TEJ, Woolrich MW, Smith SM. FSL. Neuroimage. 62(2):782-90. https://linkinghub.elsevier.com/retrieve/pii/S1053811911010603

236. Desikan RS, Ségonne F, Fischl B, Quinn BT, Dickerson BC, Blacker D, et al. An automated labeling system for subdividing the human cerebral cortex on MRI scans into gyral based regions of interest. Neuroimage. 31(3):968-80.

http://linkinghub.elsevier.com/retrieve/pii/S1053811906000437

237. Bledowski C, Prvulovic D, Hoechstetter K, Scherg M, Wibral M, Goebel $\mathrm{R}$, et al. Localizing P300 generators in visual target and distractor Processing: a combined event-related potential and functional magnetic resonance imaging study. $J$ Neurosci. 24(42):9353-60. http://www.jneurosci.org/cgi/doi/10.1523/JNEUROSCI.1897-04.2004 
238. Bachiller A, Poza J, Gómez C, Molina V, Suazo V, Hornero R. A comparative study of event-related coupling patterns during an auditory oddball task in schizophrenia. J Neural Eng. 12(1):016007. https://iopscience.iop.org/article/10.1088/1741-2560/12/1/016007

239. Lachaux JP, Rodriguez E, Martinerie J, Varela FJ. Measuring phase synchrony in brain signals. Hum Brain Mapp. 8(4):194-208.

http:/www.ncbi.nlm.nih.gov/pubmed/10619414

240. van Diessen E, Numan T, van Dellen E, van der Kooi AW, Boersma M, Hofman D, et al. Opportunities and methodological challenges in EEG and MEG resting state functional brain network research. Clin Neurophysiol. 126(8):1468-81. https://linkinghub.elsevier.com/retrieve/pii/S1388245714008104

241. Bob P, Palus M, Susta M, Glaslova K. EEG phase synchronization in patients with paranoid schizophrenia. Neurosci Lett. 447(1):73-7. https://linkinghub.elsevier.com/retrieve/pii/S0304394008013293

242. Torrence C, Compo GP, Torrence C, Compo GP. A practical guide to wavelet analysis. Bull Am Meteorol Soc. 79(1):61-78.

https://doi.org/10.1175/1520-

0477(1998)079\%3C0061:APGTWA\%3E2.0.CO;2

243. Bastos AM, Schoffelen J-M. A tutorial review of functional connectivity analysis methods and their interpretational pitfalls [Internet]. Vol. 9, Frontiers in Systems Neuroscience. p. 175. https://www.frontiersin.org/article/10.3389/fnsys.2015.00175

244. Núñez P, Poza J, Bachiller A, Gomez-Pilar J, Lubeiro A, Molina V, et al. Exploring non-stationarity patterns in schizophrenia: neural reorganization abnormalities in the alpha band. J Neural Eng. 14(4):046001. https://iopscience.iop.org/article/10.1088/1741-2552/aa6e05

245. Suo X, Lei D, Li L, Li W, Dai J, Wang S, et al. Psychoradiological patterns of small-world properties and a systematic review of connectome studies of patients with 6 major psychiatric disorders. $J$ Psychiatry Neurosci. 43(6):427. http://jpn.ca/43-6-416/

246. Jones DK, Cercignani M. Twenty-five pitfalls in the analysis of diffusion MRI data. NMR Biomed. 23(7):803-20. http://doi.wiley.com/10.1002/nbm.1543

247. Ellison-Wright I, Bullmore E. Meta-analysis of diffusion tensor imaging studies in schizophrenia. Schizophr Res. 108(1-3):3-10. http://linkinghub.elsevier.com/retrieve/pii/S0920996408005276

248. Patel S, Mahon K, Wellington R, Zhang J, Chaplin W, Szeszko PR. A meta-analysis of diffusion tensor imaging studies of the corpus callosum in schizophrenia. Schizophr Res. 129(2-3):149-55. http://linkinghub.elsevier.com/retrieve/pii/S0920996411001629

249. Hercher C, Chopra V, Beasley CL. Evidence for morphological alterations in prefrontal white matter glia in schizophrenia and bipolar disorder. $J$ Psychiatry Neurosci. 39(6):376-85. http://jpn.ca/vol39-issue6/39-6-376/ 
250. Griffa A, Baumann PS, Ferrari C, Do KQ, Conus P, Thiran J-P, et al. Characterizing the connectome in schizophrenia with diffusion spectrum imaging. Hum Brain Mapp. 36(1):354-66. http://doi.wiley.com/10.1002/hbm.22633

251. van den Heuvel MP, Sporns O, Collin G, Scheewe T, Mandl RCW, Cahn $\mathrm{W}$, et al. Abnormal rich club organization and functional brain dynamics in schizophrenia. JAMA Psychiatry. 70(8):783.

http://archpsyc.jamanetwork.com/article.aspx?doi=10.1001/jamapsychiatr y.2013.1328

252. Barysheva M, Jahanshad N, Foland-Ross L, Altshuler LL, Thompson PM. White matter microstructural abnormalities in bipolar disorder: a whole brain diffusion tensor imaging study. NeuroImage Clin. 2:558-68. https://www.sciencedirect.com/science/article/pii/S2213158213000375

253. Lynall M-E, Bassett DS, Kerwin R, McKenna PJ, Kitzbichler M, Muller $\mathrm{U}$, et al. Functional connectivity and brain networks in schizophrenia. $J$ Neurosci. 30(28):9477-87. http://www.jneurosci.org/cgi/doi/10.1523/JNEUROSCI.0333-10.2010

254. Brandl F, Avram M, Weise B, Shang J, Simões B, Bertram T, et al. Specific substantial dysconnectivity in schizophrenia: a transdiagnostic multimodal meta-analysis of resting-state functional and structural MRI studies. Biol Psychiatry. https://www.sciencedirect.com/science/article/pii/S000632231832081X

255. Nenadić I, Hoof A, Dietzek M, Langbein K, Reichenbach JR, Sauer H, et al. Diffusion tensor imaging of cingulum bundle and corpus callosum in schizophrenia vs. bipolar disorder. Psychiatry Res Neuroimaging. 266:96100. https://linkinghub.elsevier.com/retrieve/pii/S0925492716303651

256. Gildengers AG, Butters MA, Aizenstein HJ, Marron MM, Emanuel J, Anderson SJ, et al. Longer lithium exposure is associated with better white matter integrity in older adults with bipolar disorder. Bipolar Disord. 17(3):248-56. http://doi.wiley.com/10.1111/bdi.12260

257. Başar-Eroglu C, Demiralp T. Event-related theta oscillations: an integrative and comparative approach in the human and animal brain. Int $J$ Psychophysiol. 39(2-3):167-95. https://linkinghub.elsevier.com/retrieve/pii/S0167876000001409

258. Northoff G. Beyond the Passive/Active Dichotomy: A Spectrum Model of the Brain's Neural Activities. En: The Spontaneous Brain. The MIT Press; p. 3-25.

259. von Stein A, Chiang C, König P. Top-down processing mediated by interareal synchronization. Proc Natl Acad Sci U S A. 97(26):14748-53. http://www.pnas.org/cgi/doi/10.1073/pnas.97.26.14748

260. Devrim M, Demiralp T, Ademoglu A, Kurt A. A model for P300 generation based on responses to near-threshold visual stimuli. Cogn Brain Res. 8(1):37-43.

https://linkinghub.elsevier.com/retrieve/pii/S0926641099000075 
261. Pomarol-Clotet E, Salvador R, Sarró S, Gomar J, Vila F, Martínez Á, et al. Failure to deactivate in the prefrontal cortex in schizophrenia: dysfunction of the default mode network? Psychol Med. 38(8):1185-93. https://www.cambridge.org/core/product/identifier/S0033291708003565/t ype/journal_article

262. Whitfield-Gabrieli S, Thermenos HW, Milanovic S, Tsuang MT, Faraone S V, McCarley RW, et al. Hyperactivity and hyperconnectivity of the default network in schizophrenia and in first-degree relatives of persons with schizophrenia. Proc Natl Acad Sci U S A. 106(4):1279-84. www.pnas.org/cgi/content/full/

263. Pomarol-Clotet E, Moro N, Sarró S, Goikolea JM, Vieta E, Amann B, et al. Failure of de-activation in the medial frontal cortex in mania: evidence for default mode network dysfunction in the disorder. World $J$ Biol Psychiatry. 13(8):616-26. http://www.tandfonline.com/doi/full/10.3109/15622975.2011.573808

264. Uhlhaas PJ, Singer W. Oscillations and neuronal dynamics in schizophrenia: the search for basic symptoms and translational opportunities. Biol Psychiatry. 77(12):1001-9. http://dx.doi.org/10.1016/j.biopsych.2014.11.019

265. Buckner RL, DiNicola LM. The brain's default network: updated anatomy, physiology and evolving insights. Nat Rev Neurosci. 20(10):593-608. http://dx.doi.org/10.1038/s41583-019-0212-7

266. Raichle ME, MacLeod AM, Snyder AZ, Powers WJ, Gusnard DA, Shulman GL. A default mode of brain function. Proc Natl Acad Sci. 98(2):676-82. http://www.pnas.org/cgi/doi/10.1073/pnas.98.2.676

267. Canali P, Sarasso S, Rosanova M, Casarotto S, Sferrazza-Papa G, Gosseries $\mathrm{O}$, et al. Shared reduction of oscillatory natural frequencies in bipolar disorder, major depressive disorder and schizophrenia. $J$ Affect Disord. 184:111-5. http://dx.doi.org/10.1016/j.jad.2015.05.043

268. Harmon-Jones E, Abramson LY, Nusslock R, Sigelman JD, Urosevic S, Turonie LD, et al. Effect of bipolar disorder on left frontal cortical responses to goals differing in valence and task difficulty. Biol Psychiatry. 63(7):693-8.

https://linkinghub.elsevier.com/retrieve/pii/S0006322307007524

269. Nusslock R, Harmon-Jones E, Alloy LB, Urosevic S, Goldstein K, Abramson LY. Elevated left mid-frontal cortical activity prospectively predicts conversion to bipolar I disorder. J Abnorm Psychol. 121(3):592601. http://doi.apa.org/getdoi.cfm?doi=10.1037/a0028973

270. Koller-Schlaud K, Ströhle A, Bärwolf E, Behr J, Rentzsch J. EEG frontal asymmetry and theta power in unipolar and bipolar depression. $J$ Affect Disord. 276:501-10. https://doi.org/10.1016/j.jad.2020.07.011

271. Baradits M, Kakuszi B, Bálint S, Fullajtár M, Mód L, Bitter I, et al. Alterations in resting-state gamma activity in patients with schizophrenia: a high-density EEG study. Eur Arch Psychiatry Clin Neurosci. 269(4):429-37. http://link.springer.com/10.1007/s00406-018-0889-z 


\section{ANEXOS}





\section{Anexo 1}

\section{Structural connectivity in schizophrenia and bipolar disorder: Effects of chronicity and antipsychotic treatment ${ }^{1}$}

Benjamín Cea-Cañas ${ }^{a}$, Rodrigo de Luis $^{b}$, Alba Lubeiro ${ }^{c}$, Javier Gomez-Pilar ${ }^{d}$, Eva Sotelo ${ }^{\mathrm{e}}$, Pilar del Valle ${ }^{\mathrm{e}}$, Marta Gómez-García ${ }^{\mathrm{e}}$, Adrián Alonso-Sánchez ${ }^{\mathrm{e}}$, Vicente Molina ${ }^{c, e, f *}$

${ }^{a}$ Clinical Neurophysiology Service, Clinical Hospital of Valladolid, Ramón y Cajal, 3, 47003 Valladolid, Spain

${ }^{b}$ Imaging Processing Laboratory, University of Valladolid, Paseo de Belén, 15, 47011 Valladolid, Spain

${ }^{c}$ Psychiatry Department, School of Medicine, University of Valladolid, Av. Ramón y Cajal, 7, 47005 Valladolid, Spain

${ }^{d}$ Biomedical Engineering Group, University of Valladolid, Paseo de Belén, 15, 47011 Valladolid, Spain

${ }^{e}$ Psychiatry Service, Clinical Hospital of Valladolid, Ramón y Cajal, 3, 47003 Valladolid, Spain

${ }^{f}$ Neurosciences Institute of Castilla y León (INCYL), Pintor Fernando Gallego, 1, 37007 University of Salamanca, Spain

*Corresponding author.

Highlights

- Structural connectivity is altered in both chronic schizophrenia and bipolar disorder.

- Structural connectivity is not altered in first-episode schizophrenia.

- Network parameters does not differ between patients receiving or not antipsychotics.

- Executive functions and social cognition are related to small-world index in the schizophrenia group.

\footnotetext{
${ }^{1}$ Received 20 November 2018; Received in revised form 11 February 2019; Accepted 14 February 2019.

https://doi.org/10.1016/j.pnpbp.2019.02.006
} 


\section{ABSTRACT}

Previous studies based on graph theory parameters applied to diffusion tensor imaging support an alteration of the global properties of structural connectivity network in schizophrenia. However, the specificity of this alteration and its possible relation with chronicity and treatment have received small attention. We have assessed small-world (SW) and connectivity strength indexes of the structural network built using fractional anisotropy values of the white matter tracts connecting 84 cortical and subcortical regions in 25 chronic and 18 first episode (FE) schizophrenia and 24 bipolar patients and 28 healthy controls. Chronic schizophrenia and bipolar patients showed significantly smaller SW and connectivity strength indexes in comparison with controls and FE patients. SW reduction was driven by increased averaged path-length (PL) values. Illness duration but not treatment doses were negatively associated with connectivity strength, SW and PL in patients. Bipolar patients exposed to antipsychotics did not differ in SW or connectivity strength from bipolar patients without such an exposure. Executive functions and social cognition were related to SW index in the schizophrenia group. Our results support a role for chronicity but not treatment in structural network alterations in major psychoses, which may not differ between schizophrenia and bipolar disorder, and may hamper cognition.

Keywords: Small-world, Structural connectivity, Schizophrenia, Bipolar disorder, Fractional anisotropy, Antipsychotic agents.

\section{Introduction}

The mental functions altered in major psychoses are likely sustained by the coordinated activity of distributed cerebral regions. Thus, analyses of global connectivity patterns are potentially relevant for the assessment of the underpinnings of psychoses. Analyses derived from graph-theory combined with structural and functional imaging techniques have been helpful in describing global connectivity in the normal brain (Sporns et al., 2004) and its alterations in major psychoses (van den Heuvel et al., 2010; Wang et al., 2012).

Among parameters derived from graph-theory, the small-world index (SW) is useful to summarize properties of the global brain network. This index, in reference to the small-world architecture of the human brain (Bassett et al., 2008), measures the balance between local connectivity or specialization of neuronal assemblies and the optimal communication between distant brain regions in a functional way. Since it is believed that the brain architecture must simultaneously coordinate the information transfer of local specialized networks and global functioning, SW can be seen as a measure of overall efficiency of the functional brain network. Thus, SW is the ratio between 
clustering coefficient $(\mathrm{CLC})$ and characteristic path length $(\mathrm{PL})$, as indexes of the local and long-range connectivity, respectively. High SW values are therefore associated with efficient coordination of distributed cerebral subnetworks. Additionally, the connectivity strength parameter can estimate the average connective density among the network nodes. Thus, SW and connectivity strength indexes respectively allow for more qualitative and quantitative depictions of the structural connective architecture of the brain.

When applied to structural connectivity in schizophrenia, graph-based analyses have revealed a pattern of decreased integration secondary to higher PL (van den Heuvel et al., 2010), associated with altered SW properties (Suo et al., 2018). Other connectivity alterations described in this syndrome included increased CLC (Bassett et al., 2008) and reduced global efficiency (Wang et al., 2012).

Most of these studies were performed in chronic patients. Therefore, a role for chronicity and/or treatment in these connectivity alterations cannot be discarded. To our knowledge, only one study evaluated complex network organization in medication-naïve, first episode schizophrenia patients (Zhang et al., 2015), revealing longer PL but conserved SW values.

Besides, global characteristics of structural networks have not been directly compared between schizophrenia and other major psychoses using graph parameters. Such comparisons could inform about the possible specificity of global network dysconnectivity in schizophrenia. In bipolar disorder, reduced overall CLC and increased PL of structural connectivity have been described in comparison to healthy subjects (Leow et al., 2013). Later studies used different methods and measurements, such as modelling cortical and subcortical areas and testing connections between these areas (Forde et al., 2015) and assessing rich-club connectivity (Collin et al., 2016). The diversity of parameters across studies is a further difficulty for the assessment of the effects of syndrome and chronicity. To our knowledge, the only published face to face comparison of brain networks between schizophrenia and bipolar disorder of structural connectivity is based on correlations of cortical thickness (Wheeler et al., 2015). Other studies have compared cerebral connectivity between schizophrenia and bipolar disorder, although not using graph theory. In a recent preprint, Ji et al. showed widespread fractional anisotropy (FA) reductions in schizophrenia and bipolar disorder as well as higher FA in schizophrenia in areas overlapping the default mode network. Moreover, functional data revealed both increased and decreased connectivity across cerebral regions in schizophrenia compared with control subjects, with intermediate differences in bipolar patients (Skatun et al., 2016).

Therefore, we decided to compare global structural connectivity properties in schizophrenia (chronic and first episode), bipolar patients and control subjects, using diffusion magnetic resonance imaging (dMRI) and the same graph parameters in all groups. Our objectives were to assess the 
specificity and the possible effects of chronicity and antipsychotic treatment on structural connectivity networks. Thus, we selected schizophrenia and bipolar patients with comparable illness duration (to assess specificity) and compared FE and chronic schizophrenia patients (to assess chronicity effects). Since a proportion of bipolar patients receive long-term antipsychotic therapy, we planned to compare patients with and without such treatment (to assess possible effects of these drugs on global networks). Our hypotheses were that (i) network alterations would be more evident in schizophrenia than in bipolar patients, (ii) similar alterations would be found in chronic and FE schizophrenia patients and (iii) antipsychotic treatment would be unrelated to these alterations.

\section{Methods}

\subsection{Subjects}

Forty-three schizophrenia ( 25 males) and 24 euthymic type I bipolar (13 males) patients, and 28 healthy subjects (19 males) were included. The schizophrenia sample included 25 stable and 18 first-episode patients (11 males).

Two of the psychiatrists in the group (VM and PdV) diagnosed the patients according to the Diagnostic and Statistical Manual of Mental Disorders, 5th edition. Diagnoses were based on full interviews with patients and other relevant sources of information, such as interviews with relatives.

Schizophrenia patients received stable doses of atypical antipsychotics, 31 of them in monotherapy (12 of them also received antidepressants and 7 benzodiazepines). FE patients had been receiving stable doses of antipsychotics for $<15$ days. All the bipolar patients receiving stable treatment: 13 were treated with antipsychotics, and 11 were not receiving this treatment nor had received it for at least the last six months (none of the latter had ever received antipsychotics for more than one month). Fifteen bipolar patients were being treated with lithium, seven with anticonvulsants and nine with antidepressants at the time of inclusion. In total, four bipolar patients were treated in monotherapy with lithium, and 20 received a combination treatment. Antipsychotic doses were individually transformed to chlorpromazine equivalents in $\mathrm{mg} / \mathrm{d}$, and adherence was assessed by means of the percent of antipsychotic prescriptions withdrawn during the last six months from pharmacy offices according to the regional register system.

Positive symptoms were scored using the Positive and Negative Syndrome Scale (PANSS) (Kay et al., 1987) and negative symptoms with the Brief Negative Symptoms Scale (Mané et al., 2014). All schizophrenia patients showed positive and negative psychotic symptoms, while in the bipolar group five cases showed positive symptoms and twelve showed negative symptoms 
at the time of inclusion. Exclusion criteria were: (i) any neurological illness; (ii) history of cranial trauma with loss of consciousness longer than one minute; (iii) past or present substance abuse, except nicotine or caffeine; (iv) total intelligence quotient (IQ) smaller than 70; and (iv) for patients, any other psychiatric disorder, and (v) for controls, any current psychiatric or neurological diagnosis or treatment.

The population here included overlaps in part with that of previous reports of our group in schizophrenia on functional networks based on evoked response (Gomez-Pilar et al., 2017), graph complexity (Gomez-Pilar et al., 2018) and structural connectivity of specific tracts of the prefrontal region (Molina et al., 2017).

We obtained written informed consent from all participants after full printed information. The ethical committees of the Clinical University Hospital of Valladolid approved the study.

\subsection{Cognitive assessment}

We assessed cognition in patients and controls using the Wechsler Adult Intelligence Scale, WAIS-III (IQ); the Wisconsin Card Sorting Test (WCST; completed categories and percentage of perseverative errors); and the Spanish version of the Brief Assessment in Cognition in Schizophrenia Scale (BACS) (Segarra et al., 2011). In this battery, verbal memory is assessed using word lists, working memory using digit span tests, motor speed by simultaneously placing with both hands two small tokens in a recipient, verbal fluency as the average of words beginning by " $\mathrm{s}$ " and " $\mathrm{f}$ " and belonging to a semantic category, performance speed with a digit/symbols tests and problem solving with a Tower of London test. Social cognition was assessed using Mayer, Salovey and Caruso Emotional Intelligence Test (MSCEIT), which scores the dimensions of emotional perception, facilitation, understanding and management (Mayer et al., 2003).

\subsection{MRI acquisition and processing}

All the acquisitions were performed at a Philips Achieva 3 Tesla MRI unit (Philips Healthcare, Best, The Netherlands) located at the University of Valladolid. The protocol (total acquisition time was $18 \mathrm{~min}$ ) included an anatomical T1-weighted image and a diffusion acquisition. For the T1weighted images, a turbo field echo (TFE) sequence was employed, using a spatial resolution of $1 \times 1 \times 1 \mathrm{~mm}^{3}$ and a matrix size of $256 \times 256.160$ sagittal slices were acquired, covering the whole brain.

With regard to the diffusion weighted images (DWIs), an EPI acquisition was employed, obtaining 61 images with different gradient directions (b- 
value $=1000 \mathrm{~s} / \mathrm{mm}^{2}$ ) and one baseline image. Matrix size was $128 \times 128$, with a voxel size of $2 \times 2 \times 2 \mathrm{~mm}^{3}$. 66 axial slices were acquired.

From the raw data, a processing pipeline was applied in order to extract a connectivity matrix from each subject. The pipeline, which is explained in deeper detail elsewhere (Molina et al., 2017), makes use of freely available research-oriented software (FSL, Freesurfer and MRtrix) in order to first obtain a tissue-type segmentation, a cortical parcellation and a segmentation of subcortical gray matter structures from the T1-weighted images. This information is combined with the diffusion acquisition to obtain a whole brain, anatomically-constrained tractography ( 2 million streamlines were generated for each subject).

Finally, connectivity matrices were constructed from the tractography results and the (registered) cortical parcellation. Connectivity between any two cortical regions is described in terms of the averaged FA (fractional anisotropy) found along the streamlines connecting them. FA is a commonly employed diffusion metric, and quantifies how much the diffusion is predominantly oriented along one direction. FA is usually interpreted as a descriptor of white matter integrity, as several alterations (axonal destruction or demyelination, for instance) have been described to yield lower FA values.

Image processing is summarized in Fig. 1.

As 84 regions were employed for cortical parcellation, $84 \times 84$ symmetric connectivity matrices were obtained. No threshold was applied to the matrices, but matrix coefficients can be equal to zero if no streamlines are found between any two cortical regions.

\subsection{Graph-theory parameters}

From the structural connectivity matrices, we calculated three graph parameters (Rubinov and Sporns, 2010): (i) connectivity strength (i.e. mean network node degree), (ii) network segregation using CLC, and (iii) network integration by means of PL. In addition, SW index was also computed as a useful description of the topology of the whole network. Thus, whereas connectivity strength is as a quantitative marker of global connectivity, SW gives a measure of the network efficiency, yielding complementary information of the structural network.

In order to obtain results independent of network size and network strength, SW index was computed as the ratio between normalized CLC and PL. For that purpose, an ensemble of 50 surrogate random networks was obtained by random reshuffling of the connections (Stam et al., 2009). Then, $C L C_{n}$ and $P L_{n}$ were defined as follows (Stam et al., 2009):

$$
\begin{aligned}
& C L C_{n}=\frac{\mathrm{CLC}}{\mathrm{C}^{\text {random }}}, \\
& P L_{n}=\frac{\mathrm{PL}}{\mathrm{L}^{\text {random }}},
\end{aligned}
$$


where $\mathrm{C}^{\text {random }}$ and $\mathrm{L}^{\text {random }}$ represent the average of the $\mathrm{CLC}$ and $\mathrm{PL}$ values over the 50 surrogate networks. Finally, SW index is the ratio between the aforementioned $\mathrm{CLC}_{n}$ and $\mathrm{PL}_{n}$.

It is noteworthy that the weights of the connectivity matrices represent the averaged FA along the streamlines connecting two brain regions.

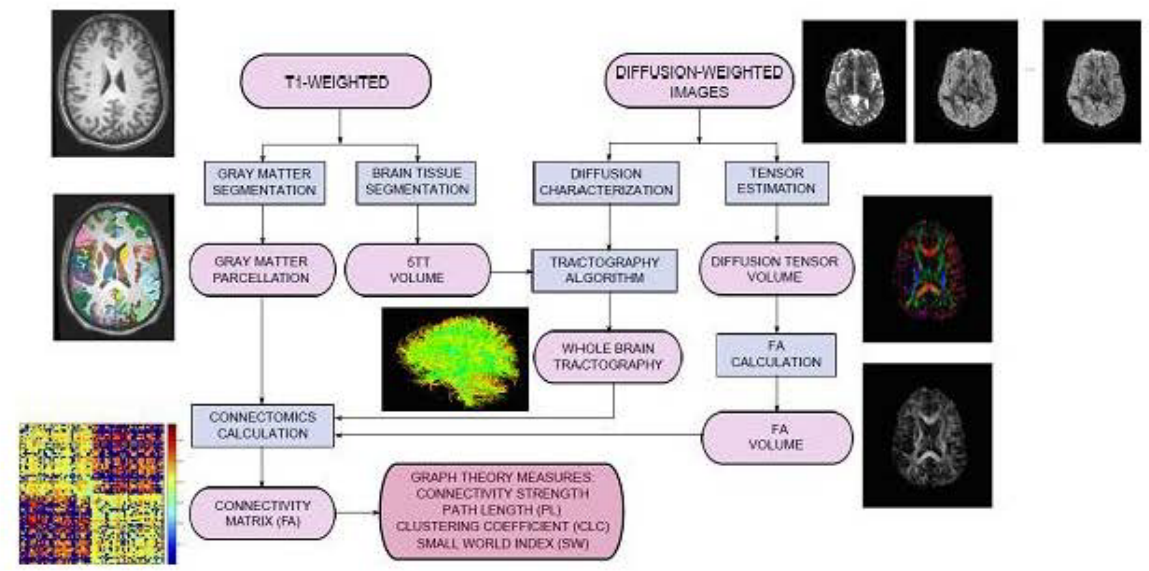

Fig 1. Schem atic depiction of the processing pipeline. Pink boxes indicate initial data, as well as intermediate and final processing outputs. Blue boxes indicate procedures that are applied to the data. From the T1-weighted im ages, different gray matter structures were segmented to obtain a gray matter parcellation. Also, gray matter, white matter and CSF were separated, and further combined to form the so-called "five-tissue-type" image (5tt), which is employed to guide the anatomically constrained tractography algorithm. Tractography is performed also using the diffusion MRI data, which is employed as well to create fractional anisotropy (FA) maps. All this information (gray matter parcellation, whole-brain tractography and FA $m$ ap) is taken into account to perform the connectom ics calculation, which yields a connectivity m atrix. From this matrix, different graph theory parameters are extracted that characterize the connectivity net work.

\subsection{Statistical analyses}

As a general strategy, to reduce type I errors risk we analyzed a parameter summarizing network architecture (SW) and another one summarizing connective density (connectivity strength). If SW showed significant between-groups differences, we carried out further analyses (PL and $(\mathrm{LC})$ to characterize network alterations. We chose non-parametric statistics given the subgroups sample sizes. Therefore, in the study, $p$ values $<.05$ were taken as significant since independent analyses with one or a small number of comparisons were carried out to test a priori hypothesis, as follows:

Our first aim was to analyze the specificity of structural network alterations described in schizophrenia. For this purpose, we compared two values (SW and connectivity strength) values between chronic schizophrenia and bipolar patients and healthy controls using a single Kruskall-Wallis test. 
Only if a significant effect of group was detected, pots-hoc comparisons using Mann-Whitney tests were performed to identify which groups differed in SW and connectivity strength values. Next, since $S W$ is the ratio between $C L C_{n}$ and $P L_{n}$, we planned to compare these values between the groups showing significant SW differences.

Our second aim was to assess the role of chronicity in these structural connectivity alterations. This effect was tested using a single Mann-Whitney $U$ test between chronic and FE patients for SW and connectivity strength. Then, Spearman's rho coefficients were calculated between graph parameters and, duration of illness (in months).

Finally, to further assess the possible effects of antipsychotics on brain connectivity, we compared with a Mann-Whitney test the network values in the bipolar group between patients receiving $(n=13)$ or not $(n=11)$ antipsychotics during at least the last six months. Moreover, we calculated in the Spearman's rho coefficients between current antipsychotic dosage and network values.

Finally, we calculated the Spearman's rho coefficients between SW values and cognitive performance (BACS, perseverative errors in WCST and social cognition) and symptoms scores (PANSS positive and total, and BNSS total).

For the sake of interpretability of network values, we assessed the Pearson's correlation coefficients with Bonferroni adjustment between structural SW and the average FA values in white matter tracts connecting prefrontal cortex (PFC) with other relevant regions in accordance with the methodology employed in a previous study (Molina et al., 2017).

A database with the main data supporting the present results is freely available (Mendeley Data, v2 http://dx.doi.org/10.17632/y93pffg4zd).

\section{Results}

Demographic data are shown in Table 1. Sex distribution did not differ between groups $\left(\chi^{2}=1.428, d f=3, p=.699\right)$. Both schizophrenia groups and bipolar patients showed a generalized cognitive deficit as compared to controls. Negative symptoms scores were significantly higher in schizophrenia as compared to bipolar patients (Table 1 ).

There was a significant difference in age between groups $\left(\chi^{2}=27.13\right.$, $\mathrm{df}=3, \mathrm{p}<.001$ ), bipolar patients being significantly older than FE SZ ( $U=34.5$, $z=4.53, p<.001)$ and healthy controls $(U=139, z=3.47, p<.001)$. Chronic SZ patients were also significantly older that FE SZ patients $(U=84.5, z=3.22$, $p<.001)$, but both SZ groups did not differ significantly in age from healthy controls.

Illness duration was significantly longer in chronic schizophrenia $(U=14.5, z=4.66, p<.001)$ and bipolar $(U=14, z=4.68, p<.001)$ as 
compared to FE patients, but did not differ between the former two groups ( $U=204, z=0.891, p=.372)$.

Treatment adherence was deemed to be good in patients. All groups withdrew $>70 \%$ of prescription from pharmacy offices during the last six months.

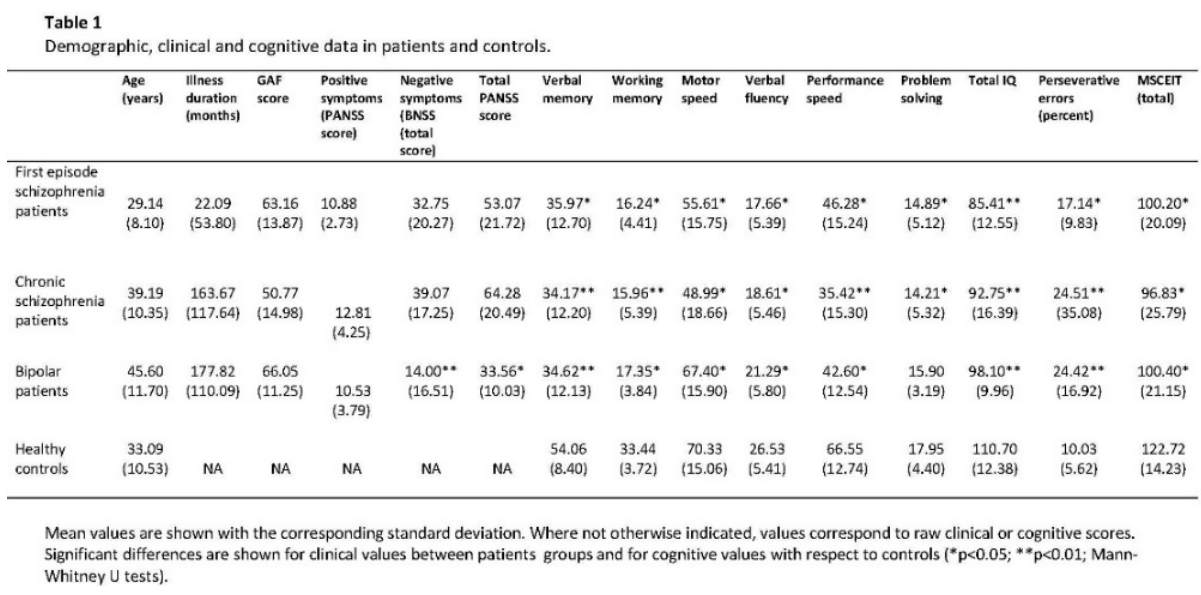

\subsection{SW values in specific tracts}

SW values were directly related to FA in the following tracts linking homolateral regions $(n=85$; in all cases $p<.001)$ : right superior frontal with superior parietal $(r=0.429)$; right rostral middle frontal with hippocampus $(r=0.330)$ and superior temporal $(r=0.351)$; left superior frontal with superior parietal $(r=0.332)$; left middle frontal with caudate $(r=0.318)$ and superior parietal $(r=0.429)$. Therefore, higher SW values were associated with higher FA values in the tracts linking prefrontal cortex with other regions.

\subsection{Specificity analyses}

A significant effect of group (chronic SZ, bipolar, control) was found on SW $(\chi 2=6.096, d f=2, p=.042)$. Post-hoc Mann-Whitney tests showed significantly lower SW values in chronic SZ as compared to controls ( $U=212$, $z=-2.459, p=.014)$, without significant differences in SW between chronic SZ and bipolar patients $(U=255, z=-0.900, p=.368$; Table 2 and Fig. 2$)$. 


\section{Anexo 1}

Table 2

Structural connectivity parameters for patients and controls.

\begin{tabular}{lcccc}
\hline & $\begin{array}{c}\text { First episode } \\
\text { schizophrenia } \\
\text { patients }\end{array}$ & $\begin{array}{c}\text { Chronic } \\
\text { schizophrenia } \\
\text { patients }\end{array}$ & $\begin{array}{c}\text { Bipolar } \\
\text { patients }\end{array}$ & $\begin{array}{c}\text { Healthy } \\
\text { controls }\end{array}$ \\
\hline Clustering coefficient & $0.996(0.001)$ & $0.994(0.003)$ & $0.994(0.004)$ & $0.996(0.002)$ \\
Path Length & $1.014(0.005)$ & $1.021(0.011)^{*}$ & $1.018(0.010)^{*}$ & $1.014(0.005)$ \\
Small-worldness & $0.982(0.005)$ & $0.974(0.012)^{*}$ & $0.976(0.012)^{*}$ & $0.982(0.006)$ \\
$\begin{array}{l}\text { Connectivity Strength } \\
\text { (Density) }\end{array}$ & $0.341(0.019)$ & $0.311(0.037)^{* *}$ & $0.304(0.035)^{* *}$ & $0.343(0.030)$ \\
\hline
\end{tabular}

Significant differences in comparison to healthy controls ${ }^{*} p<0.05 ; * * p<0.01$.
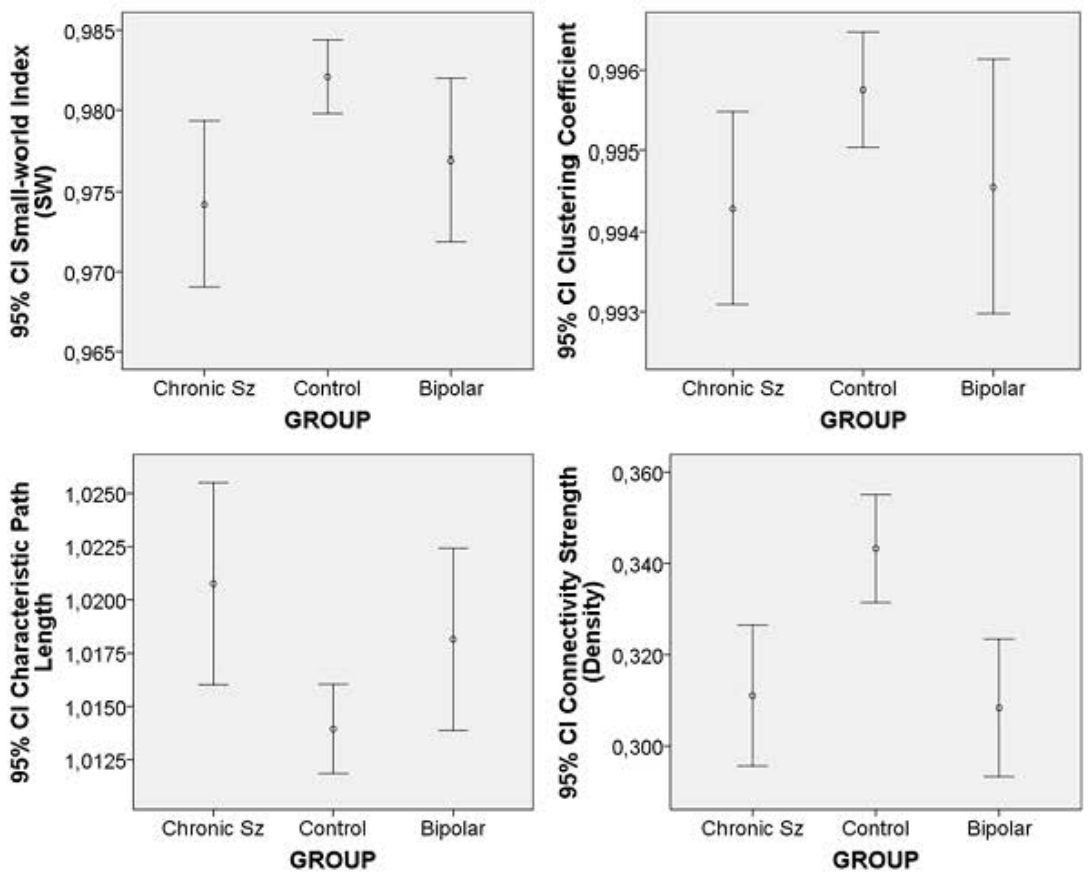

Fig. 2. $95 \%$ d intervals of network parameters for structural connectivity in the chronic schizophrenia and bipolar and healthy control groups. Both patients groups showed smaller $S W$ and larger PL values than controls, without significant differences in $\mathrm{QC}$ as compared to controls. There were no significant differences in any parameter bet ween chronic $S z$ and bipolar patients.

A larger $\mathrm{PL}_{n}$ in chronic $\mathrm{SZ}$ as compared to controls $(\mathrm{U}=227, \mathrm{z}=2.192$, $p=.014)$ seems to be driving the $S W$ differences. There were no significant differences in $\mathrm{CLC}_{n}$ or $\mathrm{PL}_{n}$ between chronic $\mathrm{SZ}$ and bipolar patients. $\mathrm{CLC}_{n}$ did not differ significantly between chronic schizophrenia with respect to bipolar patients and controls. 
Similarly, a highly significant effect of group was found for connectivity strength $\left(\chi^{2}=16.391, \mathrm{df}=2, p<.001\right)$. Post-hoc Mann-Whitney tests showed significantly lower connectivity strength in chronic $S Z$ as compared to controls $(U=161, z=-3.367, p=.001)$, without significant differences between chronic SZ and bipolar patients $(U=279, z=-0.420, p=.368$; Fig. 2).

\subsection{Chronicity analyses}

Significantly lower SW values were found in chronic SZ as compared to FE patients $(U=128, z=-2.388, p=.017)$, as well as significantly longer $P L_{n}$ $(U=143, z=2.043, p=.04)$ and smaller $C_{C L}(U=135, z=-2.21, p=.027)$ (Fig. 3). There were no significant SW differences between FE patients and controls.
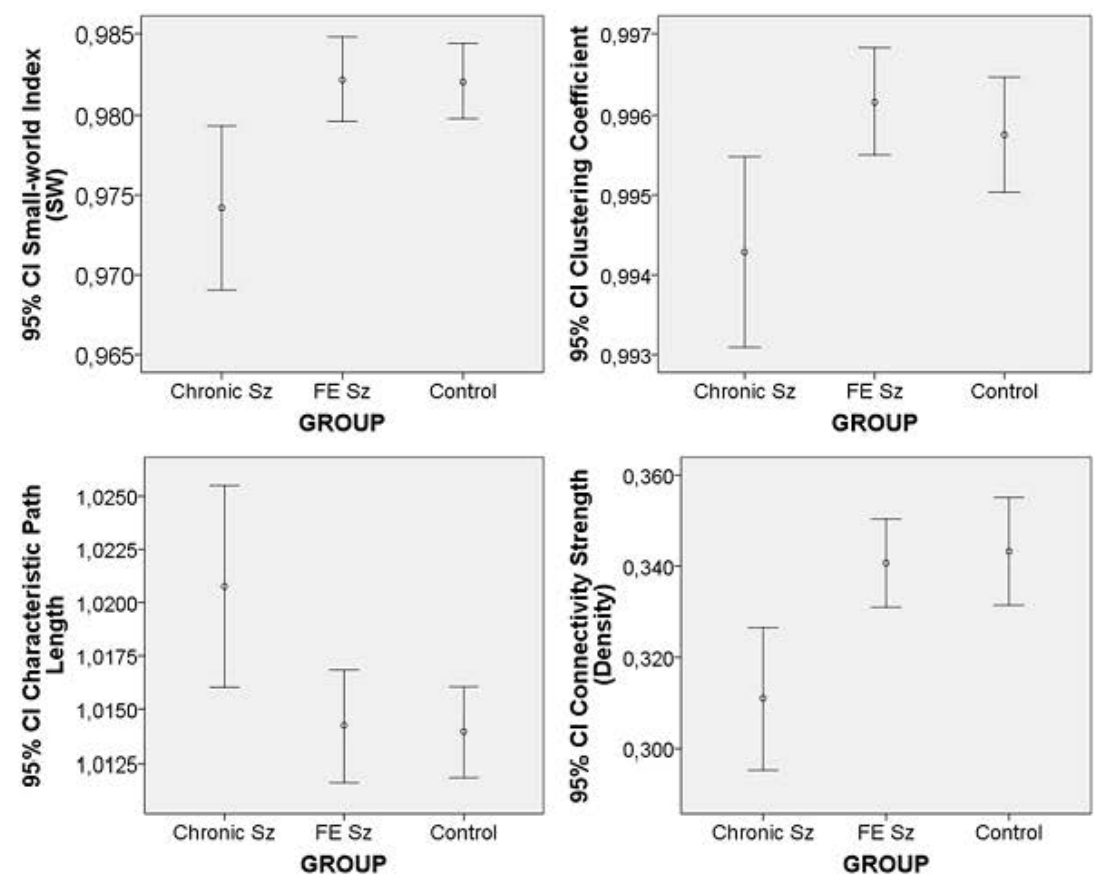

Fig. 3. $95 \%$ Q intervals of net work param eters for structural connectivity in the chronic and FE schizophrenia and healthy control groups. There were no significant differences in any param eter between FE schizophrenia and healthy controls.

Moreover, chronic patients showed significantly lower connectivity strength than FE patients $(U=103, z=-3.003, p=.003)$. FE patients did not differ in connectivity strength from healthy controls $(U=231, z=0.473$, $p=.636$ ).

Illness duration was inversely associated with SW values (rho $=-0.374$, $\mathrm{p}=.004$ ) and connectivity strength (rho $=-0.336, \mathrm{p}=.004$ ) in all patients. In the schizophrenia patients alone, this correlation was still significant for SW ( $r$ ho $=-0.515, p=.001$ ) and connectivity strength ( $r h o=-0.422, p=.009)$, but 
not in the bipolar group (SW: rho $=-0.179, \mathrm{p}=.427$; $($ rho $=-0.007, \mathrm{p}=.976)$ (Fig. 4).
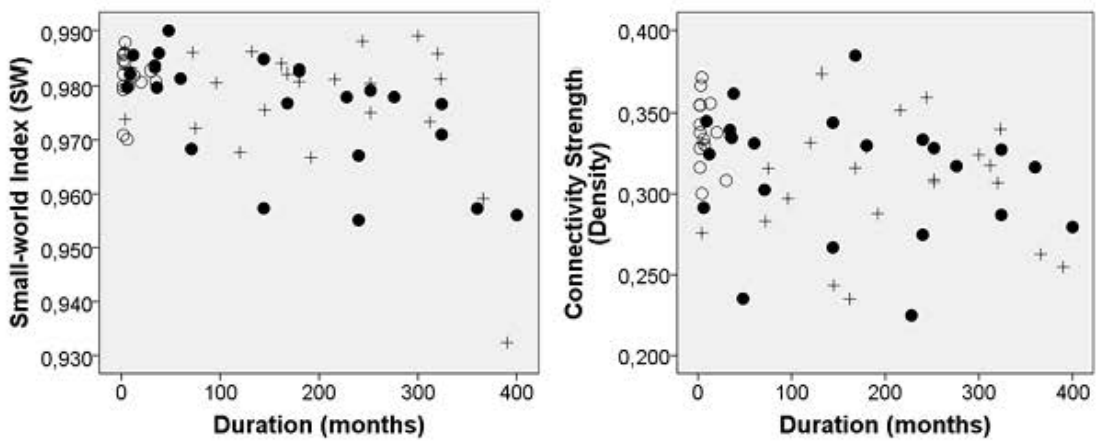

Fig. 4. Association between illness duration and SW indexes and connectivity strength values The relation was significant in the schizophrenia for both SW and connectivity strength, but not in the bipolar group. Solid dots: chronic SZ; open circles: FE schizophrenia; crosses: bipolar patients.

\subsection{Treatment effects}

Correlation coefficients between current antipsychotic doses and network parameters were not statistically significant, neither for the whole sample $(-0.059<$ rho $<0.060)$ nor for SZ and bipolar patients separately considered (0.103< rho $<0.114$; Fig. 5).
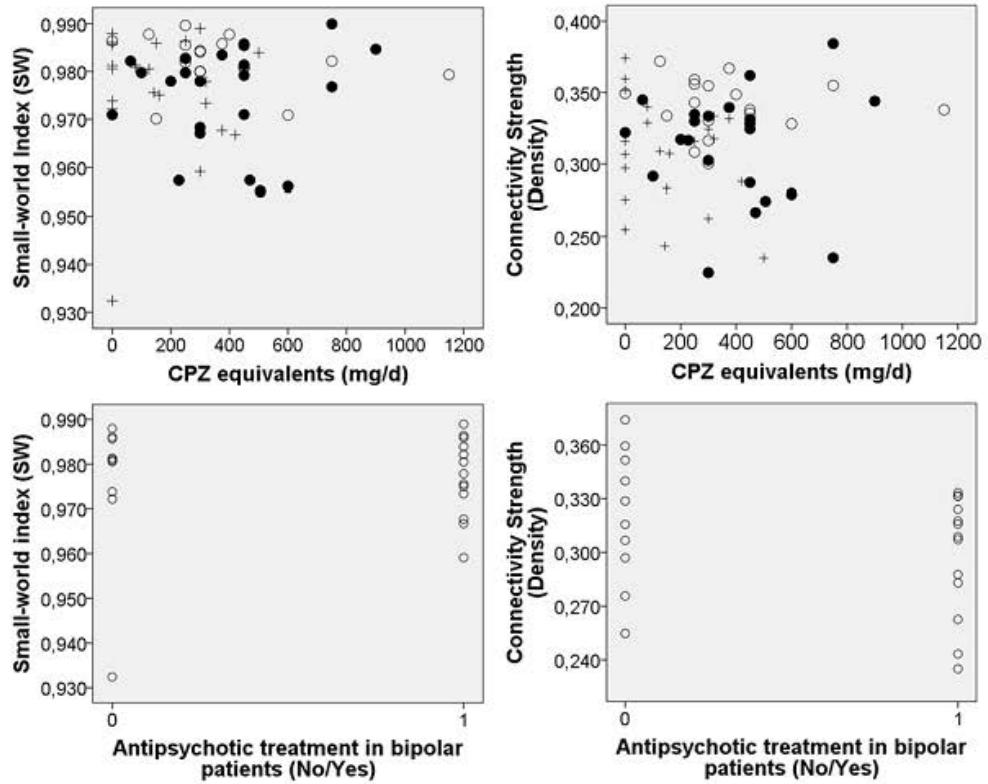

Fig. 5. In the upper row, scatterplot showingthe lack of association between antipsychotic dose and SW and connectivity strength values. (Solid dots: chronic SZ; open circles: FE schizophrenia; crosses: bipolar patients). In the lower row, comparison of $S W$ and connectivity strength values between bipolar patients with or without antipsychotic treatment. 
There were no significant differences in SW values between bipolar patients with $(0.977, \mathrm{sd} 0.008)$ and without $(0.975$, sd 0.018$)$ antipsychotic treatment exposure (Mann-Whitney, $U=57, z=-0.194, p=.875$ ). Similarly, connectivity strength did not differ between bipolar patients previously treated $(0.259$, sd 0.033$)$ and untreated $(0.320$, sd 0.037$)$ with $\operatorname{AP}(U=46$, $\mathrm{z}=-1.178, \mathrm{p}=.257)$.

Further comparisons of $\mathrm{CLC}_{n}$ (with antipsychotics (0.994, sd 0.003); without antipsychotics (0.994, sd 0.005)) and $P L_{n}$ (with antipsychotics (1.017, sd 0.007); without antipsychotics (1.019, sd 0.014)) showed non-significant differences related to exposure to antipsychotics in this group. Illness duration was not significantly different in bipolar patients treated $(210.9$, sd 96.60 months) or not $(65.29$, sd 128.67) with antipsychotics $(U=35, z=-1.04$, $\mathrm{p}=.32$ ).

\subsection{Clinical correlates}

In all patients considered together, SW values were directly associated with problem solving performance (Tower of London; rho $=0.289, p=.040$ ) and inversely to the percent of perseverative errors in WCST (rho $=-0.321$, $\mathrm{p}=.025$ ). Connectivity strength was directly associated with working memory performance (rho $=0.291, p=.039$ ).

In the SZ group, SW values were directly associated with problem solving ( $r h o=0.332, p=.048$ ), and inversely with percent of perseverative errors (rho $=-0.358, p=.035$ ). Moreover, in this group SW values were directly associated with social cognition performance (MSCEIT total score, rho $=0.372, p=.047$ ) (Fig. 6). There was a marginal association between total PANSS scores and SW values in this group ( $r h o=-0.281, p=.087$ ).
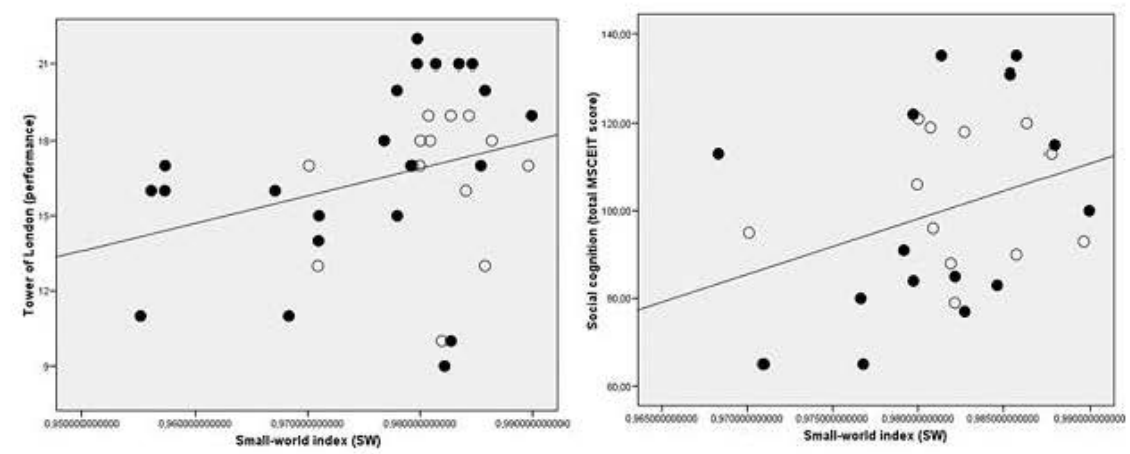

Fig. 6. Association in the schizhophrenia group between $S W$ index of strcutural connectivity and problem solving (left) and social cognition (right). Solid dots: chronic schizophrenia; open circles: FE schizophrenia. 
In this group, connectivity strength was not significantly associated with cognitive nor clinical values.

In bipolar patients, there were not significant correlations between network parameters and general cognition, but SW values were inversely related to positive symptoms ( $r h o=-0.661, p=.001$ ), and connectivity strength was related to social cognition ( $r$ o $=0.547, p=.028$ ).

\section{Discussion}

According to our data, the global architecture (SW) and density (connectivity strength) of the structural network is altered in both chronic schizophrenia and bipolar disorder, but not in FE schizophrenia. The higher PL values in chronic SZ and bipolar patients are likely to be driving their alterations of the SW.

This finding is consistent with previous reports in schizophrenia (Suo et al., 2018; van den Heuvel et al., 2010) and, according to our data, seems not to be explained by antipsychotic treatment, since there was no correlation between current antipsychotic dosage and network values, and network parameters did not differ between bipolar patients receiving or not this treatment.

As previously mentioned, there are not previous head to head comparisons of structural connectivity between schizophrenia and bipolar disorder using graph parameters and FA (Suo et al., 2018). Direct comparisons of structural connectivity based on graph parameters between these syndromes have been published based on correlations of regional gray matter thickness values, an indirect index of cortical connectivity, revealing alterations in deficit schizophrenia but not in non-deficit schizophrenia or bipolar disorder (Wheeler et al., 2015). A recent review of studies using graph theory and diffusion imaging to assess structural connectivity separately in schizophrenia and bipolar disorder supports frontal dysconnectivity in both syndromes (O'Donoghue et al., 2017).

There is evidence that FA values may be related to myelin integrity, although there are several other factors that can contribute to reductions in FA (Jones and Cercignani, 2010). Therefore, our data in chronic patients are consistent with the lower FA reported in schizophrenia (Ellison-Wright and Bullmore, 2009; Patel et al., 2011), as well as in schizophrenia and bipolar disorder (Hercher et al., 2014). The larger PL in our chronic patients is also consistent with the reported reduction of global communication paths (Griffa et al., 2015; van den Heuvel et al., 2013) in schizophrenia. Moreover, network alterations in the bipolar group also agree with previous report on widespread decease of FA in this syndrome (Barysheva et al., 2013). Taken together, these findings suggest a disruption of white matter tracts in chronic psychoses. 
Our analysis reveals an alteration in connective architecture in both schizophrenia and bipolar disorder. Our aim was to assess global properties of such architecture, not considering alteration in specific tracts, but previous literature supports a different regional distribution of connective alterations between these syndromes. A recent review based on gray matter volumes support hyperconnectivity between the default mode and limbic networks in schizophrenia as compared to bipolar disorder, as well as hypoconnectivity between limbic and salience networks (Brandl et al., 2019). We previously described FA alterations in the connections between prefrontal cortex, caudate nucleus, thalamus and anterior cingulate in schizophrenia (Molina et al., 2017), which seems consistent with the former results. Another group reported no effect of group for FA between schizophrenia and bipolar patients and controls in corpus callosum and cingulum bundle with radial diffusivity and apparent diffusion coefficient elevated in schizophrenia and not bipolar disorder (Nenadic et al., 2017). These regional differences in connectivity may underlie altered global patterns of connectivity in both psychotic syndromes.

If antipsychotic treatment is not the primary reason behind structural connectivity alteration in these syndromes, illness might have a progressively deleterious effect on that connectivity. In other words, chronicity but not antipsychotic treatment could be the primary reason of global network dysconnectivity in these syndromes, which seem consistent with the negative association found in the present sample between illness duration (but not age) and SW in our patients. Moreover, beyond illness duration, other factors might have a negative influence on cerebral connectivity in chronic psychotic patients, such as early drug consumption or social isolation. The corresponding potential effects may be assessed with specific designs.

In the bipolar group, alterations in connectivity might hypothetically be secondary to other treatments than antipsychotics, such as mood stabilizers. This however seems unlikely, since a positive relation has been reported between lithium exposure and FA values (Gildengers et al., 2015), and the majority of the bipolar patients were receiving lithium.

In the schizophrenia group, higher SW values were associated with a better performance in executive (Tower of London and WCST) and social cognition tasks, which seems consistent with the broad cortical involvement in this kind of tasks, given the multiple cognitive demands underlying it. Thus, when connectivity architecture is hampered, performance may decrease due to a lower capacity for harmonizing the activity of the multiple areas involved. In the bipolar group, however, we did not detect a similar relation between network structure and cognition. This could be related to the smaller sample size of this group but may also suggest different underpinnings for the lower $\mathrm{SW}$ in bipolar disorder as compared to schizophrenia, which is also suggested by the inverse association in this group but not in schizophrenia with positive symptoms. 
According to our data, architecture disruption (SW alterations) seems to have larger consequences for cognition in schizophrenia than quantitative (connectivity strength) deficits. This seems consistent with the data revealing a reduced betweenness centrality for frontal hubs in schizophrenia (van den Heuvel et al., 2010), since a less central role of these structures in the global network could reduce its SW value. Similar data have been reported with functional imaging network analyses (Lynall et al., 2010; van den Heuvel et al., 2013).

Our study has limitations. Bipolar patients were significantly older than chronic schizophrenia patients, but this is likely an effect of the later illness onset of bipolar disorder (Dagani et al., 2017): since we tried to collect samples with similar illness duration, an older age was expected in bipolar patients. Interhemispheric connections were not considered to assess network values. Sample sizes are relatively small, but this size is similar to that in other reports in the field, with the advantage of including bipolar and schizophrenia patients. Our sample did not include first episodes of bipolar disorder, and we lack a completely treatment-naïve schizophrenia sample, which would be necessary to clarify the possible role of treatment in network deficits.

\section{Conclusion}

Alterations of global structural connectivity were found in both chronic schizophrenia and bipolar syndromes but not in FE schizophrenia; the effect of antipsychotic treatment on structural connectivity seems questionable since no correlation was found between current antipsychotic dosage and connectivity parameters, and these did not differ between bipolar patients receiving antipsychotic treatment and those who do not. Structural connectivity values were positively associated in these syndromes to performance in working memory and problem solving tests. White matter connective alterations may have a pathogenetic role in primary psychotic syndromes.

\section{Conflict of interest}

None.

\section{Funding}

This work was supported by the Instituto Carlos III (PI15/00299) and the Gerencia Regional de Salud de Castilla y León (GRS 1485/A/17 and GRS 1721/A/18) grants; 'Consejería de Educación de la Junta de Castilla y León' (VA05P17 grant); and predoctoral fellowships to A. Lubeiro ('Consejería de 
Educación Junta de Castilla y León') and to J. Gomez-Pilar (University of Valladolid).

\section{References}

Barysheva, M., Jahanshad, N., Foland-Ross, L., Altshuler, L. L., \& Thompson, P. M. (2013). White matter microstructural abnormalities in bipolar disorder: A whole brain diffusion tensor imaging study. Neurolmage: Clinical, 2, 558-568. https://doi.org/10.1016/J.NICL.2013.03.016

Bassett, D. S., Bullmore, E., Verchinski, B. A., Mattay, V. S., Weinberger, D. R., \& Meyer-Lindenberg, A. (2008). Hierarchical Organization of Human Cortical Networks in Health and Schizophrenia. Journal of Neuroscience, 28(37), 92399248. https://doi.org/10.1523/JNEUROSCI.1929-08.2008

Brandl, F., Avram, M., Weise, B., Shang, J., Simões, B., Bertram, T., ... Sorg, C. (2018). Specific substantial dysconnectivity in schizophrenia A transdiagnostic multimodal meta-analysis of resting-state functional and structural MRI studies. Biological Psychiatry. https://doi.org/10.1016/J.BIOPSYCH.2018.12.003

Collin, G., van den Heuvel, M. P., Abramovic, L., Vreeker, A., de Reus, M. A., van Haren, N. E. M., ... Kahn, R. S. (2016). Brain network analysis reveals affected connectome structure in bipolar I disorder. Human Brain Mapping, 37(1), 122134. https://doi.org/10.1002/hbm.23017

Dagani, J., Signorini, G., Nielssen, O., Bani, M., Pastore, A., Girolamo, G. de, \& Large, M. (2017). Meta-analysis of the Interval between the Onset and Management of Bipolar Disorder. The Canadian Journal of Psychiatry, 62(4), 247-258. https://doi.org/10.1177/0706743716656607

Ellison-Wright, I., \& Bullmore, E. (2009). Meta-analysis of diffusion tensor imaging studies in schizophrenia. Schizophrenia Research, 108(1-3), 3-10. https://doi.org/10.1016/j.schres.2008.11.021

Forde, N. J., O'Donoghue, S., Scanlon, C., Emsell, L., Chaddock, C., Leemans, A., ... McDonald, C. (2015). Structural brain network analysis in families multiply affected with bipolar I disorder. Psychiatry Research: Neuroimaging, 234(1), 4451. https://doi.org/10.1016/j.pscychresns.2015.08.004

Gildengers, A. G., Butters, M. A., Aizenstein, H. J., Marron, M. M., Emanuel, J., Anderson, S. J., ... Reynolds, C. F. (2015). Longer lithium exposure is associated with better white matter integrity in older adults with bipolar disorder. Bipolar Disorders, 17(3), 248-256. https://doi.org/10.1111/bdi.12260

Gomez-Pilar, J., Lubeiro, A., Poza, J., Hornero, R., Ayuso, M., Valcárcel, C., ... Molina, V. (2017). Functional EEG network analysis in schizophrenia: Evidence of larger segregation and deficit of modulation. Progress in Neuro-Psychopharmacology and Biological Psychiatry, 76, 116-123. https://doi.org/10.1016/j.pnpbp.2017.03.004

Gomez-Pilar, J., Poza, J., Bachiller, A., Gómez, C., Núñez, P., Lubeiro, A., ... Hornero, R. (2018). Quantification of Graph Complexity Based on the Edge Weight Distribution Balance: Application to Brain Networks. International Journal of Neural Systems, 28(01), 1750032. https://doi.org/10.1142/S0129065717500320

Griffa, A., Baumann, P. S., Ferrari, C., Do, K. Q., Conus, P., Thiran, J.-P., \& Hagmann, P. (2015). Characterizing the connectome in schizophrenia with diffusion spectrum 
imaging. Human Brain Mapping, 36(1), 354-366.

https://doi.org/10.1002/hbm.22633

Hercher, C., Chopra, V., \& Beasley, C. L. (2014). Evidence for morphological alterations in prefrontal white matter glia in schizophrenia and bipolar disorder. Journal of Psychiatry \& Neuroscience : JPN, 39(6), 376-385. Retrieved from http://www.ncbi.nlm.nih.gov/pubmed/24936776

Jones, D. K., \& Cercignani, M. (2010). Twenty-five pitfalls in the analysis of diffusion MRI data. NMR in Biomedicine, 23(7), 803-820. https://doi.org/10.1002/nbm.1543

Kay, S. R., Fiszbein, A., \& Opler, L. A. (1987). The positive and negative syndrome scale (PANSS) for schizophrenia. Schizophrenia Bulletin, 13(2), 261-276. Retrieved from http://www.ncbi.nlm.nih.gov/pubmed/3616518

Leow, A., Ajilore, O., Zhan, L., Arienzo, D., GadElkarim, J., Zhang, A., ... Altshuler, L. (2013). Impaired inter-hemispheric integration in bipolar disorder revealed with brain network analyses. Biological Psychiatry, 73(2), 183-193. https://doi.org/10.1016/j.biopsych.2012.09.014

Lynall, M.-E., Bassett, D. S., Kerwin, R., McKenna, P. J., Kitzbichler, M., Muller, U., \& Bullmore, E. (2010). Functional Connectivity and Brain Networks in Schizophrenia. Journal of Neuroscience, 30(28), 9477-9487. https://doi.org/10.1523/JNEUROSCI.0333-10.2010

Mané, A., García-Rizo, C., Garcia-Portilla, M. P., Bergé, D., Sugranyes, G., GarciaAlvarez, L., ... Fernandez-Egea, E. (2014). Spanish adaptation and validation of the Brief Negative Symptoms Scale. Comprehensive Psychiatry, 55(7), 1726-1729. https://doi.org/10.1016/j.comppsych.2014.05.024

Mayer, J. D., Salovey, P., Caruso, D. R., \& Sitarenios, G. (2003). Measuring emotional intelligence with the MSCEIT V2.0. Emotion (Washington, D.C.), 3(1), 97-105. Retrieved from http://www.ncbi.nlm.nih.gov/pubmed/12899321

Molina, V., Lubeiro, A., Soto, O., Rodriguez, M., Álvarez, A., Hernández, R., \& de LuisGarcía, R. (2017). Alterations in prefrontal connectivity in schizophrenia assessed using diffusion magnetic resonance imaging. Progress in Neuro-

Psychopharmacology \& Biological Psychiatry, 76, 107-115. https://doi.org/10.1016/j.pnpbp.2017.03.001

Nenadić, I., Hoof, A., Dietzek, M., Langbein, K., Reichenbach, J. R., Sauer, H., \& Güllmar, D. (2017). Diffusion tensor imaging of cingulum bundle and corpus callosum in schizophrenia vs. bipolar disorder. Psychiatry Research. Neuroimaging, 266, 96-100. https://doi.org/10.1016/j.pscychresns.2017.05.011

O'Donoghue, S., Holleran, L., Cannon, D. M., \& McDonald, C. (2017). Anatomical dysconnectivity in bipolar disorder compared with schizophrenia: A selective review of structural network analyses using diffusion MRI. Journal of Affective Disorders, 209, 217-228. https://doi.org/10.1016/j.jad.2016.11.015

Patel, S., Mahon, K., Wellington, R., Zhang, J., Chaplin, W., \& Szeszko, P. R. (2011). A meta-analysis of diffusion tensor imaging studies of the corpus callosum in schizophrenia. Schizophrenia Research, 129(2-3), 149-155. https://doi.org/10.1016/j.schres.2011.03.014

Rubinov, M., \& Sporns, O. (2010). Complex network measures of brain connectivity: Uses and interpretations. Neurolmage, 52(3), 1059-1069. https://doi.org/10.1016/j.neuroimage.2009.10.003 
Segarra, N., Bernardo, M., Gutierrez, F., Justicia, A., Fernadez-Egea, E., Allas, M., ... Keefe, R. S. E. (2011). Spanish validation of the Brief Assessment in Cognition in Schizophrenia (BACS) in patients with schizophrenia and healthy controls. European Psychiatry, 26(2), 69-73. https://doi.org/10.1016/j.eurpsy.2009.11.001 Skåtun, K. C., Kaufmann, T., Tønnesen, S., Biele, G., Melle, I., Agartz, I., ... Westlye, L. T. (2016). Global brain connectivity alterations in patients with schizophrenia and bipolar spectrum disorders. Journal of Psychiatry \& Neuroscience : JPN, 41(5), 331-341. https://doi.org/10.1503/jpn.150159

Sporns, O., Chialvo, D. R., Kaiser, M., \& Hilgetag, C. C. (2004). Organization, development and function of complex brain networks. Trends in Cognitive Sciences, 8(9), 418-425. https://doi.org/10.1016/j.tics.2004.07.008

Stam, C. J., de Haan, W., Daffertshofer, A., Jones, B. F., Manshanden, I., van Cappellen van Walsum, A. M., ... Scheltens, P. (2009). Graph theoretical analysis of magnetoencephalographic functional connectivity in Alzheimer's disease. Brain, 132(1), 213-224. https://doi.org/10.1093/brain/awn262

Suo, X., Lei, D., Li, L., Li, W., Dai, J., Wang, S., ... Gong, Q. (2018). Psychoradiological patterns of small-world properties and a systematic review of connectome studies of patients with 6 major psychiatric disorders. Journal of Psychiatry \& Neuroscience : JPN, 43(5), 170214. Retrieved from http://www.ncbi.nlm.nih.gov/pubmed/29952747

van den Heuvel, M. P., Mandl, R. C. W., Stam, C. J., Kahn, R. S., \& Hulshoff Pol, H. E. (2010). Aberrant Frontal and Temporal Complex Network Structure in Schizophrenia: A Graph Theoretical Analysis. Journal of Neuroscience, 30(47), 15915-15926. https://doi.org/10.1523/JNEUROSCI.2874-10.2010

van den Heuvel, M. P., Sporns, O., Collin, G., Scheewe, T., Mandl, R. C. W., Cahn, W., ... Kahn, R. S. (2013). Abnormal rich club organization and functional brain dynamics in schizophrenia. JAMA Psychiatry, 70(8), 783-792. https://doi.org/10.1001/jamapsychiatry.2013.1328

Wang, Q., Su, T.-P., Zhou, Y., Chou, K.-H., Chen, I.-Y., Jiang, T., \& Lin, C.-P. (2012). Anatomical insights into disrupted small-world networks in schizophrenia. Neurolmage, 59(2), 1085-1093. https://doi.org/10.1016/j.neuroimage.2011.09.035

Wheeler, A. L., Wessa, M., Szeszko, P. R., Foussias, G., Chakravarty, M. M., Lerch, J. P., ... Voineskos, A. N. (2015). Further neuroimaging evidence for the deficit subtype of schizophrenia: a cortical connectomics analysis. JAMA Psychiatry, 72(5), 446-455. https://doi.org/10.1001/jamapsychiatry.2014.3020

Zhang, R., Wei, Q., Kang, Z., Zalesky, A., Li, M., Xu, Y., ... Huang, R. (2015). Disrupted brain anatomical connectivity in medication-naïve patients with first-episode schizophrenia. Brain Structure and Function, 220(2), 1145-1159. https://doi.org/10.1007/s00429-014-0706-z 



\section{Anexo 2}

\section{Connectivity strength of the EEG functional network in schizophrenia and bipolar disorder ${ }^{2}$}

Benjamín Cea-Cañas ${ }^{a}$, Javier Gomez-Pilar ${ }^{b}$, Pablo Núñez ${ }^{b}$, Eva RodríguezVázquez $^{c}$, Nieves de Uribe ${ }^{c}$, Álvaro Díez ${ }^{d}$, Adela Pérez-Escudero ${ }^{c}$, Vicente Molina ${ }^{c, d, e}{ }^{*}$

${ }^{a}$ Clinical Neurophysiology Service. Clinical Hospital of Valladolid, Ramón y Cajal, 3, 47003 Valladolid, Spain.

${ }^{b}$ Biomedical Engineering Group, University of Valladolid, Paseo de Belén, 15, 47011 Valladolid, Spain.

${ }^{c}$ Psychiatry Service, Clinical Hospital of Valladolid, Ramón y Cajal, 3, 47003 Valladolid, Spain.

${ }^{d}$ Psychiatry Department, School of Medicine, University of Valladolid, Av. Ramón y Cajal, 7, 47005 Valladolid, Spain.

${ }^{e}$ Neurosciences Institute of Castilla y León (INCYL), School of Medicine, University of Valladolid, Av. Ramón y Cajal, 7, 47005 Valladolid, Spain.

*Corresponding author.

Highlights

- Connectivity strength CS assesses the functional connectivity of brain networks.

- Global band pre-stimulus CS values are specifically higher in schizophrenia.

- Pharmacological treatment is not associated with the altered CS values.

- CS values in schizophrenia are associated with worse general cognition.

\section{ABSTRACT}

The application of graph theory measures in the study of functional brain networks allows for the description of their general properties and their

\footnotetext{
${ }^{2}$ Received 3 June 2019; received in revised form 16 September 2019; Accepted 29 October 2019.

https://doi.org/10.1016/j.pnpbp.2019.109801
} 
alterations in mental illness. Among these measures, connectivity strength (CS) estimates the degree of functional connectivity of the whole network. Previous studies in schizophrenia patients have reported higher baseline CS values and modulation deficits in EEG spectral properties during cognitive activity. The specificity of these alterations and their relationships with pharmacological treatments remain unknown. Therefore, in the present study, we assessed functional CS on EEG-based brain networks in 79 schizophrenia and 29 bipolar patients in addition to 63 healthy controls. The subjects performed a P300 task during the EEG recordings from which the prestimulus and the task-related modulation CS values were computed in the global and theta bands. These values were compared between the groups and between the patients who had and had not received different treatments. The global band pre-stimulus CS was significantly higher in the schizophrenia group compared with the bipolar and control groups. Theta band CS modulation was decreased in schizophrenia and bipolar patients. Treatment with antipsychotics, lithium, benzodiazepines, and anticonvulsants did not significantly alter these CS values. The first-episode and chronic schizophrenia patients did not show significant differences in CS values. Higher global band pre-stimulus CS values were associated with worse general cognition in schizophrenia patients. These data support increased connectivity in the whole-brain network that is specific to schizophrenia and suggest a general hyper-synchronized basal state that might hamper cognition in this syndrome.

Keywords: Connectivity strength, Functional network, Graph-theory, Schizophrenia, Bipolar disorder

\section{Introduction}

Parameters derived from graph theory have been applied to biological signals to assess brain network properties in healthy subjects and those with pathological conditions (Gomez-Pilar et al., 2017; Rubinov et al., 2009; van den Heuvel et al., 2008; van den Heuvel et al., 2010). Electroencephalography (EEG) has been widely used to this end, since its temporal resolution is adequate to evaluate the fast changes in these networks that are linked to cognitive function.

Given the likely widespread cerebral involvement in mental functions, assessing the functional connectivity of whole-brain networks and its modulation by cognition is relevant to the study of mental disorders. Thus, our group has previously calculated the small-world (SW) index from EEG recordings to assess network characteristics during cognitive activity (i.e., during a P300 task performance) and has reported that, compared with controls, a lower increase in this index occurs in schizophrenia patients in association with task performance (Gomez-Pilar et al., 2017). 
In this line, other network measures can increase our understanding of functional alterations in mental disease. Among these, connectivity strength (CS), which is an extension of the density measure in binary networks, summarizes the averaged edge values of all of the nodes in the network. These edges are usually calculated from phase-locking values (PLV) that in the case of EEG indicates the degree of synchronization among sensors because a higher synchronization is associated with greater PLV among sensors.

CS represents a quantitative index of synchrony among neural assemblies. Since this synchronous firing of transitory neural assemblies is related to the interneuron mediated inhibitory neural activity (Buzsáki, 2006), from a theoretical perspective it could be proposed that possible CS alterations might relate to an abnormal excitatory/inhibitory balance. Because deficits in inhibitory transmission have been reported in major psychoses (Fee et al., 2017; Gonzalez-Burgos et al., 2011), CS alterations may be expected in these disorders.

Our group has previously reported higher pre-stimulus CS values in the EEG global and theta bands in schizophrenia (Gomez-Pilar et al., 2018a). Of these, the increase in the theta band was related to decreased modulation of the spectral properties during a P300 task in schizophrenia, defining modulation as the difference between the pre-stimulus and the response windows values (Gomez-Pilar et al., 2018b). The decreased task-related modulation of spectral properties is a finding that has been replicated in two different schizophrenia patient samples (Bachiller et al., 2014; Molina et al., 2018). Task-related modulation is likely to reflect a re-shaping in the functional connections between distributed neural assemblies, considering the role proposed for these assemblies in cognition (Buzsaki and Draguhn, 2004; Varela et al., 2001), and the fact that the evolving EEG record results from the coordinated action of such assemblies (Uhlhaas and Singer, 2010). Thus, the task-related modulation of network parameters may be proposed to reflect the re-shaping of the underlying neural assemblies related to the corresponding cognitive activity.

To our knowledge, the specificity of these CS alterations described in schizophrenia and the possible effects of antipsychotic treatment have not yet been assessed. To this end, in the present study we assessed the CS values that were altered in our previous reports in a partially new schizophrenia sample and in a bipolar sample using a denser EEG array (Gomez-Pilar et al., 2018b). Based on these previous reports, and in order to reduce the number of comparisons, in this study we only focused on theta and global frequency bands. Our primary hypothesis was that pre-stimulus CS would be specifically higher in the schizophrenia group, and this group would exhibit lower taskrelated CS modulation that would be correlated with cognitive deficits. The inclusion of bipolar subjects also allowed us to assess the effects of antipsychotic treatment through the comparison between drug-free and 
medicated patients. In this case, our hypothesis was that the network alteration would not depend on antipsychotic treatment.

\section{Subjects and methods}

\subsection{Subjects}

In the present study, we included 79 schizophrenia (48 males) and 29 bipolar patients (17 males) in addition to 63 healthy controls ( 32 males). The schizophrenia group included 31 first-episode (FE) patients. Data from 38 healthy controls and 31 schizophrenia patients ( $7 \mathrm{FE}$ ) have been included in previous reports from our group (Gomez-Pilar et al., 2018a, Gomez-Pilar et al., $2018 b)$. None of the bipolar subjects have been included in any previous report, although diffusion tensor imaging data from 24 of these subjects have been published elsewhere (Cea-Cañas et al., 2019). All participants provided written informed consent after receiving full oral and printed information. The local ethical committee approved this study.

The patients were diagnosed by one of the psychiatrists in the group ( $\mathrm{V}$. M., the treating psychiatrist of most of the cases) according to the criteria of the Diagnostic and Statistical Manual of Mental Disorders, 5th edition, based on full interviews and all available evidence. The healthy controls were recruited through newspaper advertisements. The socio-demographic and clinical characteristics are presented in Table 1.

Table 1

Sociodemographic, clinical, and cognitive data of the patient and control groups. Individual cognitive scores are summarized in the general cognitive factor (see text).

\begin{tabular}{|c|c|c|c|c|}
\hline & $\begin{array}{l}\text { Schizophrenia } \\
\text { Patients }\end{array}$ & $\begin{array}{l}\text { Bipolar } \\
\text { patients }\end{array}$ & $\begin{array}{l}\text { Healthy } \\
\text { controls }\end{array}$ & Statistic ( $p$ value) \\
\hline M:F ratio & $48: 31$ & $17: 12$ & $32: 31$ & $x^{2}=2.05(p=0.358)$ \\
\hline Age (years) & $35.66(10.57)$ & $46.41(10.45) *$ & $38.19(10.14)$ & $\hat{F}=10.66(p<0.001)$ \\
\hline $\begin{array}{l}\text { Illness } \\
\text { duration (months) }\end{array}$ & $101.69(116.10)$ & $182.22(106.04)$ & N/A & $t=-2.95(p=0.004)$ \\
\hline Education (years) & $14.59(3.64)$ & $13.10(4.44)$ & $15.75(1.48)$ & $F=1.10(p=0.341)$ \\
\hline Parental education (yrs) & $12.11(4.40)$ & $9.63(3.29)$ & $9.75(2.49)$ & $F=1.81(p=0.177)$ \\
\hline Positive PANSS & $11.07(3.62)$ & $7.37(0.83)$ & N/A & $t=7.70(p<0.001)$ \\
\hline Negative PANSS & $16.50(7.26)$ & $9.05(2.48)$ & $N / A$ & $t=7.17(p<0.001)$ \\
\hline Young mania & $0.83(1.91)$ & $1.81(1.34)$ & $N / A$ & $t=0.47(p=0.435)$ \\
\hline Hamilton depression & $5.87(5.09)$ & $4.98(3.81)$ & $N / A$ & $t=0.10(p=0.811)$ \\
\hline Verbal memory & $34.72(13.44) * *$ & $36.00(9.25)$ ** & $49.12(8.37)$ & $F=27.61(p<0.001)$ \\
\hline Working memory & $16.41(4.73)$ ** & $17.58(4.03)$ ** & $21.44(3.37)$ & $F=23.84(p<0.001)$ \\
\hline Motor speed & $59.05(19.31) * *$ & $66.11(13.67)$ & $73.91(16.64)$ & $F=10.88(p<0.001)$ \\
\hline Verbal fluency & $17.13(7.10)^{* *}$ & $19.79(7.87)$ & $24.78(9.88)$ & $F=12.25(p<0.001)$ \\
\hline Processing speed & $41.94(14.59)^{* *}$ & $41.48(12.40) * *$ & $64.93(13.74)$ & $F=45.82(p<0.001)$ \\
\hline Problem solving & $16.02(4.36)$ & $15.95(3.30)$ & $17.11(3.25)$ & $F=1.42(p=0.245)$ \\
\hline General cognitive factor & $-0.84(0.89) * *$ & $-0.79(0.69) * *$ & $0.31(0.62)$ & $F=35.92(p<0.001)$ \\
\hline Total IQ & $92.06(14.73) * *$ & $98.32(10.23) * *$ & $114.02(11.44)$ & $F=44.11(p<0.001)$ \\
\hline $\mathrm{CPZ}$ equivalents (mg/d) & $411.21(377.28)$ & $157.40(152.90)$ & N/A & $t=3.35(p=0.001)$ \\
\hline P300 amplitude $(\mu \mathrm{V})$ & $1.04(0.61)$ & $1.23(0.63)$ & $1.58(0.76)$ & $F=0.68(p=0.260)$ \\
\hline P300 latency (ms) & $423.22(95.43)$ & $464.69(96.11)$ & $458.54(71.40)$ & $F=0.59(p=0.371)$ \\
\hline
\end{tabular}

Results are displayed as the mean (SD); N/A = not applicable. Statistics for the betweengroups comparisons (MANOVA or Chi tests) are shown in the right column ( $F(p)$ or $X^{2}(p)$ ) comparisons between patient groups are shown as $t(p)$.

${ }^{*} p<0.01$ in the bipolar compared with the schizophrenia and control groups.

** Significance levels for post-hoc comparisons between patient groups and controls are shown when applicable $(p<0.01)$. 
The exclusion criteria for patients and controls included a history of neurological illness (including cranial trauma with loss of consciousness), past or present substance abuse (except for nicotine and caffeine), and an intelligence quotient (IQ) below 70. Additionally, controls were excluded in cases of any current psychiatric diagnosis or treatment.

All chronic schizophrenia patients were receiving stable doses of atypical antipsychotics. Of these patients, 15 were also receiving antidepressants, and 27 were receiving benzodiazepines (BZD). The FE patients were also receiving stable doses of antipsychotics. Eleven of these patients had been so doing for fewer than 15 days. Regarding the bipolar patients, 15 were receiving atypical antipsychotics, 18 were receiving lithium, 4 were receiving anticonvulsants, 7 were receiving $B D Z$, and 6 were receiving antidepressants.

\subsection{Clinical and cognitive assessment}

The symptoms were scored using the Positive and Negative Syndrome Scale (PANSS) (Kay et al., 1987). Depression and mania were scored using the Hamilton Depression (HAM-D) and Young Mania Rating scales (YMRS). Cognitive data from the patients and controls were collected using the Wechsler Adult Intelligence Scale (third edition; WAIS-III) and the Spanish version of the Brief Assessment in Cognition in Schizophrenia Scale (BACS) (Segarra et al., 2011).

\subsection{EEG recording and processing}

\subsubsection{EEG data acquisition and preprocessing}

We recorded the EEG data using a 29-channel EEG system (BrainVision ${ }^{\circledR}$, Brain Products $\mathrm{GmbH}$ ). Electrodes were place at Fp1, Fp2, F7, F3, Fz, F4, F8, FC5, FC1, FCz, FC2, FC6, T7, C3, Cz, C4, T8, CP5, CP1, CP2, CP6, P7, P3, Pz, P4, $\mathrm{P} 8, \mathrm{O} 1, \mathrm{Oz}$ and $\mathrm{O} 2$ following the international $10-10$ system; the impedance was below $5 \mathrm{k} \Omega$, and the sampling frequency was $500 \mathrm{~Hz}$. The channels were referenced over $\mathrm{Cz}$ during acquisition and re-referenced offline to the averaged activity of all of the sensors (Bledowski et al., 2004; Gomez-Pilar et al., 2018c). We recorded 13 min of eyes-closed EEG activity during an auditory odd-ball 3-condition paradigm that presented 600 random stimuli in the sequence of a target $(500-\mathrm{Hz}$ tone, probability of 0.2$)$, distractor $(1000-\mathrm{Hz}$ tone, probability of 0.2$)$, and a standard $(2000-\mathrm{Hz}$ tone, probability of 0.6$)$ tone. Each tone lasted 50 milliseconds $(\mathrm{ms}$ ) and comprised a rise and fall time of $5 \mathrm{~ms}$ with an intensity of $90 \mathrm{~dB}$. The inter-stimulus interval randomly jittered between 1.16 and $1.44 \mathrm{~s}$. The participants were asked to press a button upon hearing targets, that were considered 'attended' when followed by a button press. Only the 'attended' target tones were considered for 
further analysis. Alertness differences were controlled for by comparing the accuracies of the target responses.

The following three-step artifact rejection algorithm was applied to minimize electrooculographic and electromyographic contamination (Bachiller et al., 2015): (i) an independent component analysis (ICA) was performed to discard noisy ICA components; (ii) the signals were divided after ICA reconstruction into trials of $1 \mathrm{~s}$ (from $300 \mathrm{~ms}$ prior to the stimulus onset to $700 \mathrm{~ms}$ after); and (iii) the trials with amplitudes that exceeded an adaptive statistical-based threshold were automatically rejected (Núñez et al., 2017). The signals were band-pass filtered between 1 and $70 \mathrm{~Hz}$, and a $50-\mathrm{Hz}$ notch filter was utilized to remove the power line artifact.

\subsubsection{EEG-based brain graphs and connectivity strength calculation}

In the construction of EEG-based brain graphs, network nodes are a mathematical representation of the EEG electrodes whereas the values of the network edges are calculated from the neural coupling between each pair of electrodes (Stam and van Straaten, 2012). This coupling can be estimated with different methods. Here, we selected the phase-locking value (PLV) across successive trials (Lachaux et al., 1999), which is sensitive to low-amplitude oscillatory EEG components (Spencer et al., 2003) in addition to nonlinearities (van Diessen et al., 2015).

The PLV, in turn, can be computed using different methodologies; we used the continuous wavelet transform (CWT) to compute the phase information from each trial (Bob et al., 2008) considering cones of influence to remove edge effects (Torrence et al., 1998).

Applying the CWT approach for the performance of filter and phase extraction in one operation, the PLV between two signals, $x(t)$ and $y(t)$, was obtained evaluating the variability of the phase difference across successive trials (Gomez-Pilar et al., 2018b; Lachaux et al., 1999):

$$
P L V_{x y}(k, s)=\frac{1}{N t}\left|\sum_{n=1}^{N} e^{\Delta \phi_{x y}(k, s, n)}\right|
$$

where $\mathrm{Nt}$ is the number of trials, $\Delta \phi x y$ is the instantaneous phase difference between the signals $x$ and $y, k$ is the time interval, and $s$ is the scaling factor of the mother wavelet.

We generated functional connectivity matrices using the PLV values. Due to the fact that no threshold was applied, these connectivity matrices ranged between 0 and $1 ; 0$ was obtained when two signals had no synchronization and 1 was obtained when two signals were perfectly synchronized.

We selected two windows from the EEG signal: (i) the pre-stimulus window, which corresponded to a period of expectation before the stimulus onset from $-300 \mathrm{~ms}$ to the stimulus onset, thereby the pre-stimulus window 
is located during the task performance and is completely different from the resting state; and (ii) the response window, which is related to the P3b response ( 150 to $450 \mathrm{~ms}$ after the stimulus onset). This procedure was applied both for the EEG theta band $(4-8 \mathrm{~Hz})$ and the global band $(1-70 \mathrm{~Hz})$, in which higher values of pre-stimulus CS have been reported in previous studies in schizophrenia (Gomez-Pilar et al., 2018a, Gomez-Pilar et al., 2018b).

Thus, adjacency matrices were computed for the pre-stimulus and the response windows. From these matrices, the connectivity strength was computed as follows:

$$
C S=\frac{\sum_{i=1}^{N} \sum_{j>i} w_{i j}}{N(N-1) / 2}
$$

where wij refers to PLV between nodes $i$ and $j$, and $N$ is the total number of nodes of the network (Gomez-Pilar et al., 2018b). Finally, task-related CS modulation was defined as the change of the CS values between the prestimulus and the response windows (i.e., CS at the response minus CS at the pre-stimulus windows, which allowed us to measure the intra-individual change of this measure secondary to the cognitive task).

\subsection{Statistical tests}

We compared the age and sex distribution between the groups using an analysis of variance (ANOVA) and chi-square (X2) tests, respectively. The normality of the distribution of the CS values in each group was tested using Kolmogorov-Smirnov tests. Treatment doses and illness duration were compared between the schizophrenia and bipolar groups using t-tests for independent samples.

We tested the primary hypothesis (i.e., the specificity of higher prestimulus CS values and the decreased task-related modulation) by comparing the pre-stimulus and the task-related modulation CS values in the global and theta band between the groups using a multivariate analysis of covariance (MANCOVA) with these CS values as the dependent variables and age as a covariate. This procedure was followed by the corresponding pairwise comparisons to determine which pairs showed significantly different values if a significant effect of group was found. Effect sizes were calculated using eta squared values.

Next, several analyses were performed to assess the effects of treatment and illness duration on the CS values that were altered in the patients, and the cognitive and clinical correlates of these alterations.

To assess the possible effects of treatment, we (i) calculated the Pearson's rho correlations between the current antipsychotic doses and the altered CS values, (ii) compared these values between the bipolar patients 
receiving and not receiving antipsychotics (because all schizophrenia patients were receiving antipsychotics), and (iii) compared these values between the patients receiving and not receiving lithium (bipolar patients only), BZD, anticonvulsants, or antidepressants. Given the small corresponding sample sizes, comparisons (ii) and (iii) were performed using Mann-Whitney $U$ tests.

To test the effects of illness duration, we planned comparisons of the altered CS values between the chronic and FE schizophrenia patients with a ttest.

Finally, to test the possible relevance of these altered CS values in the patients, we used stepwise linear regression to assess the relations between these parameters and cognitive performance and positive and negative symptoms scores. As in previous studies (Gomez-Pilar et al., 2018a), we calculated an overall score that summarized cognition by introducing the individual cognitive scores of all subjects into a principal component analysis and saved the factor scores for further analysis.

\section{Results}

There were no significant differences in the sex distribution between the groups. The bipolar patients were significantly older than the schizophrenia patients and healthy controls. The bipolar patients had received significantly smaller doses of antipsychotics and had longer duration of illness than the schizophrenia patients (Table 1).

The pre-stimulus and task-related modulation CS values in and the global and theta bands were normally distributed in each group.

The principal component analysis of the cognitive scores yielded a single factor for which all individual scores positively loaded, and this factor explained $55.40 \%$ of the variance (eigenvalue 3.34). The factor scores were saved. Compared with the healthy controls, the factor scores were significantly smaller in both groups of patients, which implied worse general cognitive performance (Table 1).

\subsection{Comparison of CS values}

\subsubsection{Between-group differences}

The multivariate effect of group was significant (Wilk's $\lambda=7.44$, $\mathrm{df}=8.318, \mathrm{p}<0.001$; eta squared $=0.158$ ). The effect of age was not significant (Wilk's $\lambda=0.81, \mathrm{df}=4.159, \mathrm{p}=0.52$; eta squared $=0.02$ ).

The between-subject effect tests revealed significant effects of group for the global band pre-stimulus CS (type III sum of squares $=0.012, F=3.30$, $\mathrm{df}=2, \quad \mathrm{p}=0.04$; eta squared $=0.04$ ) and theta band CS task-related modulation (type III sum of squares $=0.037, F=27.48$, df $=2, p<0.0001$; eta squared $=0.25$; Table 2, Fig. 1 ). 
Table 2

Connectivity strength (CS) values in the pre-stimulus window and the modulation of the $\mathrm{CS}$ in the global and theta bands. Modulation indicates the difference between the response and pre-stimulus windows during a P300 task (see text).

\begin{tabular}{lccc}
\hline & $\begin{array}{c}\text { Schizophrenia } \\
\text { Patients }\end{array}$ & $\begin{array}{c}\text { Bipolar } \\
\text { Patients }\end{array}$ & $\begin{array}{c}\text { Healthy } \\
\text { controls }\end{array}$ \\
\hline CS pre-stimulus (global band) & $0.3136(0.0438)^{\star}$ & $0.2963(0.0337)$ & $0.2981(0.0425)$ \\
CS pre-stimulus (theta band) & $0.3600(0.0448)$ & $0.3486(0.0397)$ & $0.3507(0.0364)$ \\
CS modulation (global band) & $-0.0003(0.0084)$ & $-0.0030(0.0081)$ & $0.0005(0.0124)$ \\
& & & \\
CS modulation (theta band) & $0.0195(0.0298)^{\star \star \star}$ & $0.0192(0.0236)^{\star \star \star}$ & $0.0636(0.0467)$ \\
\hline
\end{tabular}

Significant differences.

${ }^{*} p<0.05$ in the schizophrenia compared with the bipolar and control groups.

${ }^{\star \star \star} p<0.001$ in the schizophrenia and bipolar groups compared with the control group.
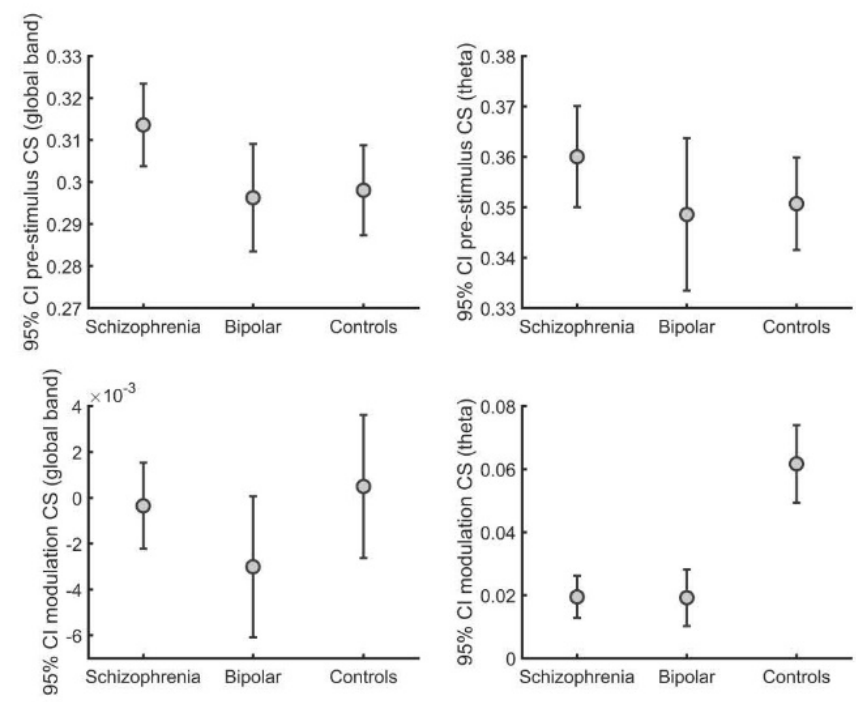

Fig. 1. Differences between groups in the pre-stimulus CS and its task-related modulation in the global band and theta bands.

Pair-wise comparisons revealed higher global band pre-stimulus CS values in the schizophrenia patients compared with the controls (mean difference $=0.016 ; 95 \% \mathrm{Cl}=0.002-0.031, \mathrm{p}=0.027$ ) and the bipolar patients (mean difference $=0.020 ; 95 \% \mathrm{Cl}=0.000-0.040, \mathrm{p}=0.049$ ). The global band pre-stimulus CS values were not significantly different in the bipolar patients compared with the healthy controls (mean difference $=-0.004 ; 95 \%$ $\mathrm{Cl}=-0.024-0.016, \quad \mathrm{p}=.70)$. There were no significant between-group differences in the pre-stimulus CS in the theta band. 
Compared with the controls, the theta band task-related CS modulation was significantly smaller in the schizophrenia patients (mean difference $=-0.043 ; 95 \% \mathrm{Cl}=-0.055$ to $-0.031, \mathrm{p}<0.001$ ) and bipolar patients (mean difference $=-0.044 ; 95 \% \mathrm{Cl}=-0.061$ to $-0.027, \mathrm{p}<0.001$ ).

Although unplanned, we explored the possible relation between the pre-stimulus global band CS and the theta-band CS modulation in the patients; the difference was significant $(r=-0.24, n=108, p=0.01)$ and indicated that larger pre-stimulus CS values were associated with smaller increases in the theta band CS with the task response.

\subsubsection{Regional analysis}

We also performed a regional analysis of the pre-stimulus and modulation CS values for both the theta band (Fig. 2) and the global band (Fig. 3). This regional analysis showed higher significant differences when comparing controls and schizophrenia patients than when comparing controls and bipolar patients.

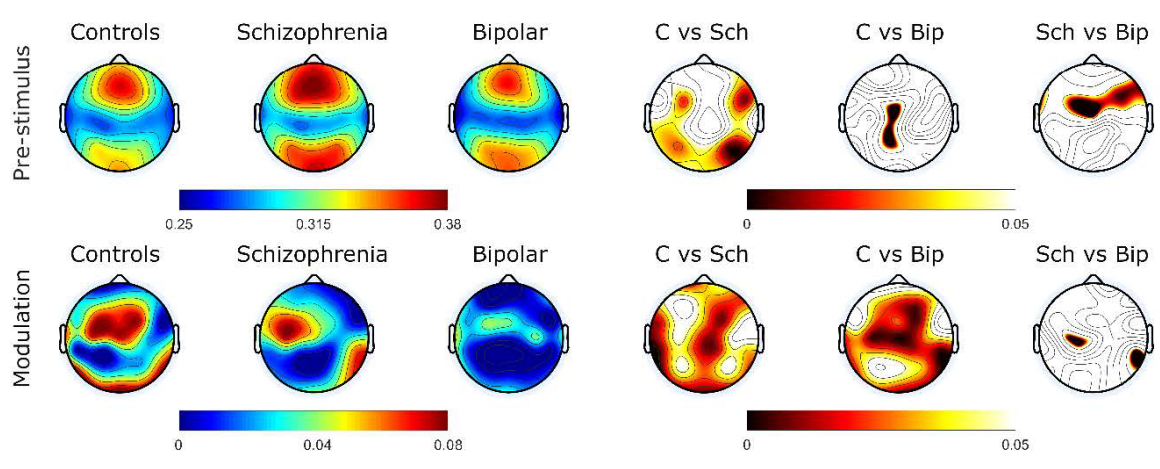

Fig. 2. Spatial distribution of the connectivity strength (CS) in the theta band for each electrode (i.e. node degree) for each group in the pre-stimulus and modulation windows (left), and the regional statistical differences between groups (right) (C: controls, Sch: schizophrenia patients, Bip: bipolar patients).

In the theta band, the main differences in the pre-stimulus window were located in the right parieto-occipital and fronto-temporal regions when comparing the control and the schizophrenia groups (Fig. 2, first row); in the modulation window, the main differences were located in the central and frontal regions for the comparison between controls and both patient groups (Fig. 2, second row). 


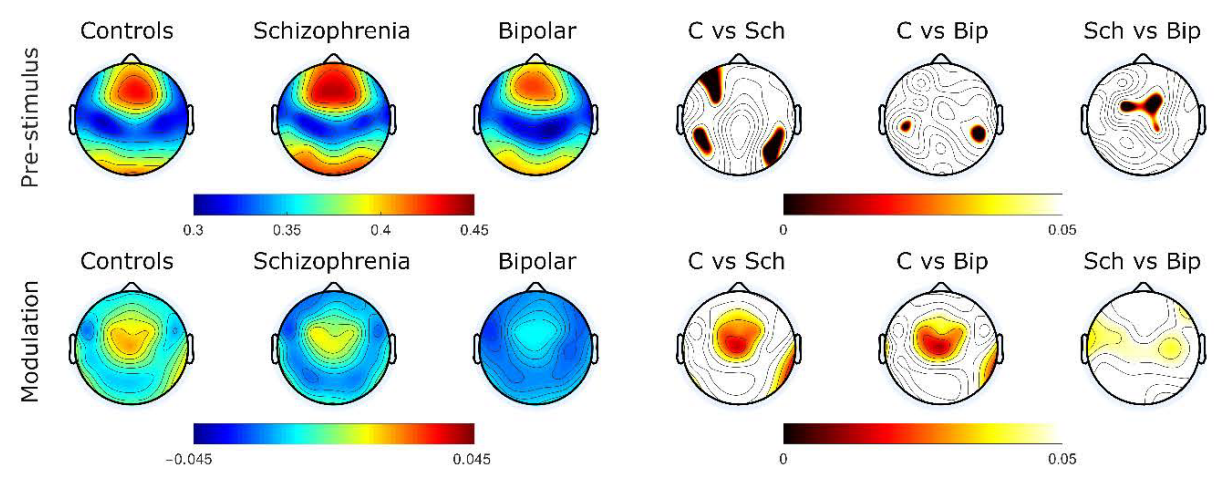

Fig. 3. Spatial distribution of the connectivity strength in the global band for each electrode (i.e. node degree) for each group in the pre-stimulus and modulation windows (left), and the regional statistical differences between groups (right) (C: controls, Sch: schizophrenia patients, Bip: bipolar patients).

In the global band, the main differences in the pre-stimulus window were located in the parietal and left frontal when comparing controls and schizophrenia patients (Fig. 3, first row); in the modulation window, the main significant differences were found in the central region for the comparison between controls and both patient groups (Fig. 3, second row).

\subsection{Treatment and illness duration effects}

The correlations of antipsychotic dose with global band pre-stimulus CS $(r=0.058, n=94, p=0.58)$ and theta band CS modulation $(r=-0.05, p=0.53)$ were not statistically significant. Likewise, these correlations were nonsignificant in the schizophrenia (global band pre-stimulus CS: $r=-0.004$; theta CS modulation $r=-0.046$ ) nor in the bipolar patients (global band pre-stimulus CS: $r=-0.025$; theta CS modulation $r=-0.008$ ).

The global band pre-stimulus CS did not differ between the bipolar patients receiving and not receiving antipsychotics $(U=84, z=-0.19, p=0.86$; Fig. 4a) or between the bipolar patients receiving and not receiving lithium $(U=73, z=-0.37, p=0.73)$. This parameter did not differ significantly between the patients receiving BZD $(U=481, z=0.69, p=0.48)$, anticonvulsants $(U=99, z=0.28, p=0.79)$, or antidepressants $(U=201$, $z=0.83, p=0.40$ ). When considering only the schizophrenia patients, there were no significant differences in global band pre-stimulus CS between subjects receiving or not $B Z D(U=84, z=-0.293, p=0.79)$ or antidepressants $(U=60, z=-1.08, p=0.29)$. 
Anexo 2

a
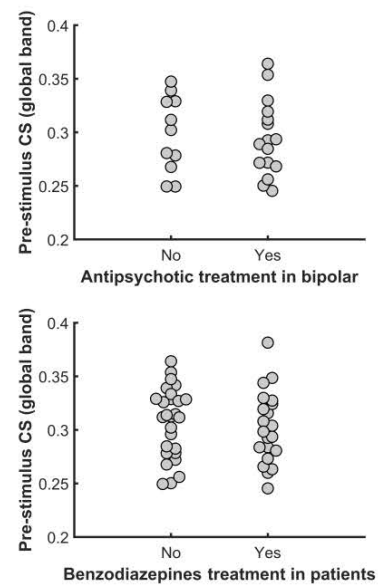

b
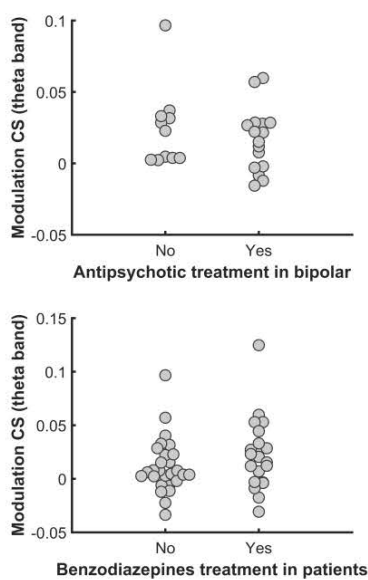
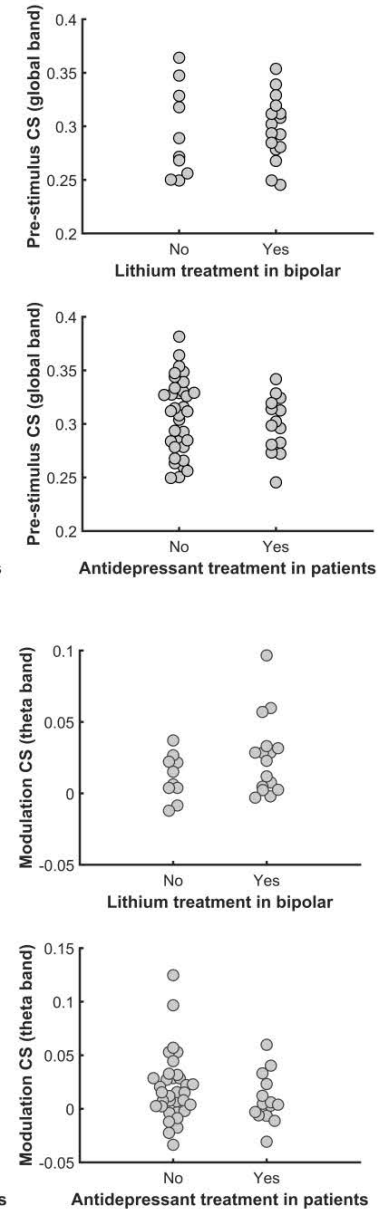

Fig. 4. (a) Individual distributions of the pre-stimulus CS in the global band in the bipolar patients receiving and not receiving antipsychotics and lithium (upper row) and the patients receiving or not receiving benzodiazepines and antidepressants (lower row). (b) Individual distributions of the theta band CS modulation in bipolar patients receiving or not receiving antipsychotics and lithium (upper row) and patients receiving or not receiving benzodiazepines and antidepressants (lower row).

Similarly, the theta band CS modulation did not differ between the bipolar patients receiving and not receiving antipsychotics $(U=70, z=-0.88$, $p=0.37$; Fig. 4 b) or between the bipolar patients receiving and not receiving lithium $(U=55, z=-1.13, p=0.18)$. This parameter did not significantly differ between the patients receiving BZD $(U=220, z=1.32, p=0.19)$, anticonvulsants $(U=84, z=-0.79, p=0.42)$, or antidepressants $(U=190$, $z=-1.09, p=0.27)$. When considering only the schizophrenia patients, there were no significant differences in theta band CS modulation between subjects receiving or not $B Z D(U=61, z=-1.41, p=0.17)$ or antidepressants $(U=59$, $z=-1.13, p=0.27)$. 
The difference in the global band pre-stimulus CS between the FE ( mean $=0.32, s d=0.06$ ) and chronic schizophrenia patients (mean $=0.31$, $s d=0.03)$ was not statistically significant $(t=1.04, p=0.29)$. Similarly, these differences were also non-significant for the theta band CS modulation (chronic $=0.016, \mathrm{sd}=0.03 ; \mathrm{FE}=0.024, \mathrm{sd}=0.03 ; \mathrm{t}=1.14, \mathrm{p}=.25$ ).

\subsection{Clinical and cognitive correlates}

Multivariate linear regression selected the global band pre-stimulus CS as a significant predictor of general cognition in the schizophrenia patients $(R 2=0.137, F=8.70, p=0.005)$. Thus, in this group, a higher global band CS in the pre-stimulus window was associated with worse cognitive performance (Fig. 5a). There were no significant predictors of general cognition in the bipolar group. For illustrative purposes, correlation coefficients were calculated between global band pre-stimulus $\mathrm{CS}$ and individual BACS domains in schizophrenia and bipolar patients (Table S1).
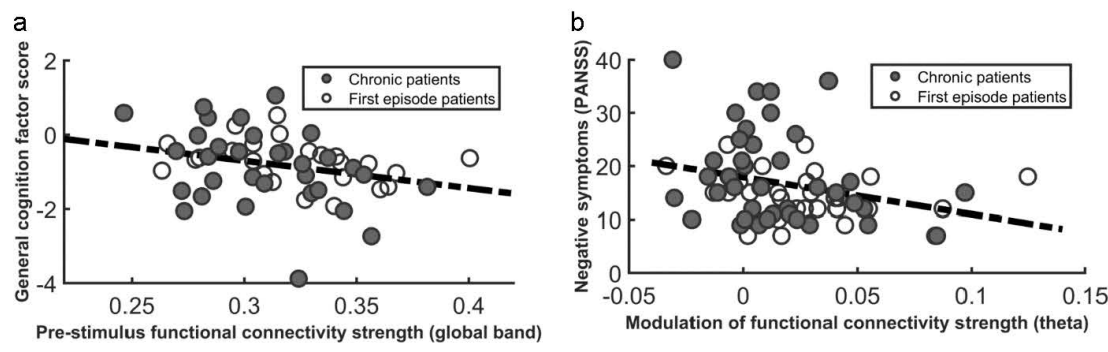

Fig. 5. Scatterplot representing (a) the association between the global-band pre-stimulus $\mathrm{CS}$ and the general cognition score in schizophrenia patients, and (b) the association between the theta band task-related CS modulation and the general cognition score in schizophrenia patients.

Moreover, linear regression selected the theta band CS modulation as a significant predictor of negative symptoms in the schizophrenia patients $(\mathrm{R} 2=0.084, \mathrm{~F}=6.23, \mathrm{p}=0.015 ; \mathrm{Fig} .5 \mathrm{~b})$. Positive symptoms were not predicted in this group by the CS values. In the bipolar group, there were no significant associations of the CS values with the PANSS scores.

Illness duration and the HAM-D, and YMRS scores were uncorrelated with the global band pre-stimulus and theta band modulation CS in all cases.

\section{Discussion}

In this study, we found a higher global band pre-stimulus CS in schizophrenia patients compared with bipolar patients and healthy controls and a highly significant deficit of theta band CS modulation (i.e., a lesser increase with task performance of the CS in this band) in both the 
schizophrenia and bipolar patients. The larger global band pre-stimulus CS in schizophrenia was associated with worse cognitive performance, and lower theta band CS modulation was associated with negative symptoms. Antipsychotics and other treatments seemed to have no significant effect on this alteration, and age did not play a major role in the CS differences between groups.

The abnormally high pre-stimulus CS found in this sample of schizophrenia patients is in line with previously reported findings with partially overlapping samples (Gomez-Pilar et al., 2018a, Gomez-Pilar et al., 2018b). We were able to replicate the finding of larger global band pre-stimulus CS (Gomez-Pilar et al., 2018a) but were unable to replicate the finding in the theta band (Gomez-Pilar et al., 2018b). In addition, we have found that the higher global band pre-stimulus CS is not regional-dependent, but shows more widespread statistical significant differences when comparing the schizophrenia patients with controls. In this context, the present data suggest that a global band pre-stimulus CS increase may be specific to schizophrenia.

We calculated the CS from the phase-locking values (PLV) between sensors. Therefore, because neurons firing within a functional assembly synchronize their signal phases, the larger pre-stimulus CS in the schizophrenia patients (i.e., higher average PLV values among the sensors) suggests a hypersynchronized state in this condition. The significant negative association between cognition and the pre-stimulus CS suggests that this potentially hyperactive basal state hampers cognitive processing. Correspondingly, the reduced increase in the theta band CS modulation implies a hyposynchronization in this band with test performance.

The most remarkable differences between the patients and controls were those of the smaller theta band CS modulation, which were common to schizophrenia and bipolar patients and likely unrelated to pharmacological treatment. This smaller modulation may be interpreted as an overall smaller increase in theta activity in patients. This assertion agrees with previous results that have demonstrated a smaller modulation in media frequencies towards slower bands and a lower increase in the relative theta power during the same task in a different schizophrenia sample (Bachiller et al., 2014) (replicated in (Molina et al., 2018)).

We cannot discard a relation between these two alterations as suggested by their significant correlation in the patients; i.e., we cannot discount the possibility that a general hyper-synchronized brain activity during the pre-stimulus window could modify the expected response to the task in the patients. This pattern would be in line with proposals of a relevant role for the basal or resting state of the brain in the stimulus-induced patterns of cerebral activity (Northoff, 2018). Theta activity increases in normal conditions during odd-ball task performance (Başar-Eroglu and Demiralp, 2001); thus, basal hyperactivity may hamper the expected pattern of brain activation 
during P300 task performance. Theta oscillations play a role in the synchronization between relatively distant regions (von Stein et al., 2000), and the dominant frequency of a neuronal assembly is dependent on the number of participating neurons so that lower frequencies involve larger assembly sizes (Buzsaki and Draguhn, 2004). Moreover, slow-band oscillations may subtend cortico-cortical interactions (Devrim et al., 1999). In this context, our findings of a higher global band pre-stimulus CS and its reduced modulation in the theta band may imply a lower capacity for general signal integration in psychoses, which could be related to negative symptoms that might be influenced by the pre-stimulus state. Finally, the greater pre-stimulus CS in schizophrenia, which is coherent with a hyper-active basal state, may agree with the GABA-transmission deficit found in this disorder (Gonzalez-Burgos et al., 2011).

Our data were measures of the whole-brain network (i.e., the CS represents averaged individual nodal degrees). Nevertheless, our regional analysis supports the idea that some regions might have prominent roles in increasing the CS. For instance, the default mode network has been reported to exhibit a hyperactive basal state and a smaller task-related deactivation in schizophrenia patients compared with healthy controls (E. Pomarol-Clotet et al., 2008; Whitfield-Gabrieli et al., 2009), although similar findings have been reported in bipolar disorder (Edith Pomarol-Clotet et al., 2012). As shown in Fig. 2, the lower modulation in theta is not regional-dependent, whereas Fig. 3 shows that the lack of modulation in the global band is associated with the central and frontal brain regions, where the modulation deficit is also prominent in the theta band. This area overlaps with the default network area, thus our results would support a hyperactive state in this network that might hamper cognitive performance and task-related modulation of cerebral activity. However, care must be taken when comparing these results because our data in both the pre-stimulus and response windows were acquired during the performance of a task while the cited functional assessments of the default mode network were performed in the resting state.

In the schizophrenia group there was an increased global band prestimulus CS and a reduced theta band CS modulation, whereas in the bipolar group, only the latter was observed. This pattern might suggest a different substrate of this modulation deficit between these groups, which could be further investigated.

Our study has limitations, most of which are related to the lack of completely treatment-free patients and the small sample of bipolar patients. Since many patients were receiving different drugs at the same time, their potential interaction was not fully controlled. Moreover, conducting connectivity analyses on scalp signals could introduce bias in the results. Thus, we checked that connections with phase differences of 0 or 180 ( \pm 1 degree) were $<1 \%$. In addition, in order to minimize volume conduction effects, we 
followed a well-known strategy based on the assumption that volume conduction similarly affects the connectivity estimates in two different experimental contrasts (Bastos and Schoffelen, 2016), so that spurious estimates can be effectively eliminated by comparing both conditions (Bastos and Schoffelen, 2016). Furthermore, source analyses would be appropriate to assess the possible roles of key regions in the overall differences. Finally, it would be of interest to assess the CS properties of the patients during the resting state (which might be different from the pre-stimulus window during task performance).

\section{Conclusion}

Connectivity strength in the functional brain network was higher in schizophrenia patients, and this difference was associated with cognitive deficits and negative symptoms. These findings may indicate a hyperactive synchronization among neural groups in schizophrenia that may hamper cognition.

The following are the supplementary data related to this article.

Table $\mathbf{~} 1$

Correlations between neurophysiological values found to be altered in the patients (global band pre-stimulus and theta modulation) and the six individual BACS domains.

\begin{tabular}{lcccc}
\hline & \multicolumn{2}{c}{ Schizophrenia patients } & \multicolumn{2}{c}{ Bipolar patients } \\
\cline { 2 - 5 } & $\begin{array}{c}\text { CS global band } \\
\text { pre-stimulus }\end{array}$ & $\begin{array}{c}\text { CS theta } \\
\text { modulation }\end{array}$ & $\begin{array}{c}\text { CS global band } \\
\text { pre-stimulus }\end{array}$ & $\begin{array}{c}\text { CS theta } \\
\text { modulation }\end{array}$ \\
\hline Verbal memory & -0.055 & 0.224 & -0.275 & 0.353 \\
Working memory & $-0.257^{\star}$ & 0.159 & -0.363 & 0.125 \\
Motor speed & $-0.359^{\star \star}$ & 0.086 & -0.119 & 0.214 \\
Verbal fluency & -0.148 & 0.050 & -0.368 & 0.031 \\
Processing speed & $-0.354^{\star \star}$ & 0.125 & -0.104 & 0.111 \\
Problem solving & $-0.310^{*}$ & $0.337^{\star *}$ & 0.283 & -0.372 \\
\hline
\end{tabular}

CS: Connectivity strength; BACS: Brief Assessment in Cognition in Schizophrenia; ${ }^{\star} \mathrm{p}<0.05 ;{ }^{\star \star} \mathrm{p}<0.01$

\section{Funding}

This work has been supported by the following grants: the "Instituto de Salud Carlos III" (PI15/00299 and PI18/00178), the "Gerencia Regional de Salud de Castilla y León" (GRS 1485/A/17 and GRS 1721/A/18), and the "Consejería de Educación de la Junta de Castilla y León" (VA059U13 and VA057P17). P. Núñez was in receipt of a predoctoral scholarship 'Ayuda para contratos predoctorales para la Formación de Profesorado Universitario (FPU)' grant from the 'Ministerio de Educación, Cultura y Deporte' (FPU17/00850). 


\section{Ethical statement for Progress in Neuro-Psychopharmacology \& Biological Psychiatry}

I testify on behalf of all co-authors that our article submitted to Progress in Neuro-Psychopharmacology \& Biological Psychiatry:

- This material has not been published in whole or in part elsewhere;

- The manuscript is not currently being considered for publication in another journal;

- All authors have been personally and actively involved in substantive work leading to the manuscript, and will hold themselves jointly and individually responsible for its content.

\section{Declaration of Competing Interest}

None.

\section{References}

Bachiller, A., Díez, A., Suazo, V., Domínguez, C., Ayuso, M., Hornero, R., ... Molina, V. (2014). Decreased spectral entropy modulation in patients with schizophrenia during a P300 task. European Archives of Psychiatry and Clinical Neuroscience, 264(6), 533-543. https://doi.org/10.1007/s00406-014-0488-6

Bachiller, A., Poza, J., Gómez, C., Molina, V., Suazo, V., \& Hornero, R. (2015). A comparative study of event-related coupling patterns during an auditory oddball task in schizophrenia. Journal of Neural Engineering, 12(1), 016007. https://doi.org/10.1088/1741-2560/12/1/016007

Başar-Eroglu, C., \& Demiralp, T. (2001). Event-related theta oscillations: an integrative and comparative approach in the human and animal brain. International Journal of Psychophysiology : Official Journal of the International Organization of Psychophysiology, 39(2-3), 167-195. Retrieved from http://www.ncbi.nlm.nih.gov/pubmed/11163896

Bledowski, C., Prvulovic, D., Hoechstetter, K., Scherg, M., Wibral, M., Goebel, R., \& Linden, D. E. J. (2004). Localizing P300 Generators in Visual Target and Distractor Processing: A Combined Event-Related Potential and Functional Magnetic Resonance Imaging Study. Journal of Neuroscience, 24(42), 9353-9360. https://doi.org/10.1523/JNEUROSCI.1897-04.2004

Bob, P., Palus, M., Susta, M., \& Glaslova, K. (2008). EEG phase synchronization in patients with paranoid schizophrenia. Neuroscience Letters, 447(1), 73-77. https://doi.org/10.1016/j.neulet.2008.09.055

Buzsaki, G., \& Draguhn, A. (2004). Neuronal Oscillations in Cortical Networks. Science, 304(5679), 1926-1929. https://doi.org/10.1126/science.1099745

Cea-Cañas, B., de Luis, R., Lubeiro, A., Gomez-Pilar, J., Sotelo, E., del Valle, P., ... Molina, V. (2019). Structural connectivity in schizophrenia and bipolar disorder: Effects of chronicity and antipsychotic treatment. Progress in Neuro- 
Psychopharmacology and Biological Psychiatry, 92, 369-377.

https://doi.org/10.1016/j.pnpbp.2019.02.006

Devrim, M., Demiralp, T., Ademoglu, A., \& Kurt, A. (1999). A model for P300

generation based on responses to near-threshold visual stimuli. Brain Research. Cognitive Brain Research, 8(1), 37-43. Retrieved from

http://www.ncbi.nlm.nih.gov/pubmed/10216272

Fee, C., Banasr, M., \& Sibille, E. (2017). Somatostatin-Positive Gamma-Aminobutyric

Acid Interneuron Deficits in Depression: Cortical Microcircuit and Therapeutic

Perspectives. Biological Psychiatry, 82(8), 549-559.

https://doi.org/10.1016/j.biopsych.2017.05.024

Gomez-Pilar, J., de Luis-García, R., Lubeiro, A., de la Red, H., Poza, J., Núñez, P., ... Molina, V. (2018a). Relations between structural and EEG-based graph metrics in healthy controls and schizophrenia patients. Human Brain Mapping, 39(8), 31523165. https://doi.org/10.1002/hbm.24066

Gomez-Pilar, J., de Luis-García, R., Lubeiro, A., de Uribe, N., Poza, J., Núñez, P., ... Molina, V. (2018b). Deficits of entropy modulation in schizophrenia are predicted by functional connectivity strength in the theta band and structural clustering. Neurolmage: Clinical, 18, 382-389. https://doi.org/10.1016/j.nicl.2018.02.005

Gomez-Pilar, J., Lubeiro, A., Poza, J., Hornero, R., Ayuso, M., Valcárcel, C., ... Molina, V. (2017). Functional EEG network analysis in schizophrenia: Evidence of larger segregation and deficit of modulation. Progress in Neuro-Psychopharmacology and Biological Psychiatry, 76, 116-123. https://doi.org/10.1016/j.pnpbp.2017.03.004

Gomez-Pilar, J., Poza, J., Bachiller, A., Gómez, C., Núñez, P., Lubeiro, A., ... Hornero, R. (2018c). Quantification of Graph Complexity Based on the Edge Weight Distribution Balance: Application to Brain Networks. International Journal of Neural Systems, 28(01), 1750032. https://doi.org/10.1142/S0129065717500320

Gonzalez-Burgos, G., Fish, K. N., \& Lewis, D. A. (2011). GABA Neuron Alterations, Cortical Circuit Dysfunction and Cognitive Deficits in Schizophrenia. Neural Plasticity, 2011. https://doi.org/10.1155/2011/723184

Kay, S. R., Fiszbein, A., \& Opler, L. A. (1987). The positive and negative syndrome scale (PANSS) for schizophrenia. Schizophrenia Bulletin, 13(2), 261-276. Retrieved from http://www.ncbi.nlm.nih.gov/pubmed/3616518

Lachaux, J. P., Rodriguez, E., Martinerie, J., \& Varela, F. J. (1999). Measuring phase synchrony in brain signals. Human Brain Mapping, 8(4), 194-208. Retrieved from http://www.ncbi.nlm.nih.gov/pubmed/10619414

Molina, V., Bachiller, A., Gomez-Pilar, J., Lubeiro, A., Hornero, R., Cea-Cañas, B., ... Poza, J. (2018). Deficit of entropy modulation of the EEG in schizophrenia associated to cognitive performance and symptoms. A replication study. Schizophrenia Research, 195, 334-342. https://doi.org/10.1016/j.schres.2017.08.057

Northoff, G. (2018). Beyond the Passive/Active Dichotomy: A Spectrum Model of the Brain's Neural Activities. In The Spontaneous Brain (pp. 3-25). The MIT Press. https://doi.org/10.7551/mitpress/11046.003.0004

Núñez, P., Poza, J., Bachiller, A., Gomez-Pilar, J., Lubeiro, A., Molina, V., \& Hornero, R. (2017). Exploring non-stationarity patterns in schizophrenia: neural reorganization abnormalities in the alpha band. Journal of Neural Engineering, 14(4), 046001. https://doi.org/10.1088/1741-2552/aa6e05 
Pomarol-Clotet, E., Salvador, R., Sarró, S., Gomar, J., Vila, F., Martínez, Á., ... McKenna, P. J. (2008). Failure to deactivate in the prefrontal cortex in schizophrenia: dysfunction of the default mode network? Psychological Medicine, 38(8), 1185-1193. https://doi.org/10.1017/S0033291708003565

Pomarol-Clotet, Edith, Moro, N., Sarró, S., Goikolea, J. M., Vieta, E., Amann, B., ... Salvador, R. (2012). Failure of de-activation in the medial frontal cortex in mania: evidence for default mode network dysfunction in the disorder. The World Journal of Biological Psychiatry, 13(8), 616-626. https://doi.org/10.3109/15622975.2011.573808

Rubinov, M., Knock, S. A., Stam, C. J., Micheloyannis, S., Harris, A. W. F., Williams, L. M., \& Breakspear, M. (2009). Small-world properties of nonlinear brain activity in schizophrenia. Human Brain Mapping, 30(2), 403-416. https://doi.org/10.1002/hbm.20517

Segarra, N., Bernardo, M., Gutierrez, F., Justicia, A., Fernadez-Egea, E., Allas, M., ... Keefe, R. S. E. (2011). Spanish validation of the Brief Assessment in Cognition in Schizophrenia (BACS) in patients with schizophrenia and healthy controls. European Psychiatry, 26(2), 69-73. https://doi.org/10.1016/j.eurpsy.2009.11.001 Spencer, K. M., Nestor, P. G., Niznikiewicz, M. A., Salisbury, D. F., Shenton, M. E., \& McCarley, R. W. (2003). Abnormal neural synchrony in schizophrenia. The Journal of Neuroscience : The Official Journal of the Society for Neuroscience, 23(19), 7407-7411. Retrieved from http://www.ncbi.nlm.nih.gov/pubmed/12917376

Stam, C. J., \& van Straaten, E. C. W. (2012). The organization of physiological brain networks. Clinical Neurophysiology, 123(6), 1067-1087. https://doi.org/10.1016/j.clinph.2012.01.011

Torrence, C., Compo, G. P., Torrence, C., \& Compo, G. P. (1998). A Practical Guide to Wavelet Analysis. Bulletin of the American Meteorological Society, 79(1), 61-78. https://doi.org/10.1175/1520-0477(1998)079<0061:APGTWA>2.0.CO;2

van den Heuvel, M P, Stam, C. J., Boersma, M., \& Hulshoff Pol, H. E. (2008). Smallworld and scale-free organization of voxel-based resting-state functional connectivity in the human brain. Neurolmage, 43(3), 528-539. https://doi.org/10.1016/j.neuroimage.2008.08.010

van den Heuvel, Martijn P., Mandl, R. C. W., Stam, C. J., Kahn, R. S., \& Hulshoff Pol, H. E. (2010). Aberrant Frontal and Temporal Complex Network Structure in Schizophrenia: A Graph Theoretical Analysis. Journal of Neuroscience, 30(47), 15915-15926. https://doi.org/10.1523/JNEUROSCI.2874-10.2010

van Diessen, E., Numan, T., van Dellen, E., van der Kooi, A. W., Boersma, M., Hofman, D., ... Stam, C. J. (2015). Opportunities and methodological challenges in EEG and MEG resting state functional brain network research. Clinical Neurophysiology, 126(8), 1468-1481. https://doi.org/10.1016/j.clinph.2014.11.018

von Stein, A., Chiang, C., \& König, P. (2000). Top-down processing mediated by interareal synchronization. Proceedings of the National Academy of Sciences of the United States of America, 97(26), 14748-14753.

https://doi.org/10.1073/pnas.97.26.14748

Whitfield-Gabrieli, S., Thermenos, H. W., Milanovic, S., Tsuang, M. T., Faraone, S. V, McCarley, R. W., ... Seidman, L. J. (2009). Hyperactivity and hyperconnectivity of the default network in schizophrenia and in first-degree relatives of persons with schizophrenia. Proceedings of the National Academy of Sciences of the United States of America, 106(4), 1279-1284. https://doi.org/10.1073/pnas.0809141106 



\title{
Anexo 3
}

\section{Altered gamma band noise power in schizophrenia and bipolar patients during a cognitive task ${ }^{3}$}

\author{
Benjamín Cea-Cañas ${ }^{a}$, Álvaro Díez ${ }^{b}$, Alba Lubeiro ${ }^{b}$, María Iglesias $^{a}$, Carmen \\ Capella ${ }^{c}$, Alberto Rodríguez-Lorenzana ${ }^{d}$, Vicente Molina ${ }^{b}, c, e^{*}$ \\ ${ }^{a}$ Clinical Neurophysiology Service. Clinical Hospital of Valladolid, Ramón y Cajal, 3, \\ 47003 Valladolid, Spain \\ ${ }^{b}$ Psychiatry Department, School of Medicine, University of Valladolid, Av. Ramón y \\ Cajal, 7, 47005 Valladolid, Spain \\ ${ }^{C}$ Psychiatry Service, Clinical Hospital of Valladolid, Ramón y Cajal, 3, 47003 \\ Valladolid, Spain \\ ${ }^{d}$ School of Psychology, Universidad de Las Américas, Quito, Ecuador \\ ${ }^{e}$ Neurosciences Institute of Castilla y León (INCYL), School of Medicine, University of \\ Valladolid, Av. Ramón y Cajal, 7, 47005 Valladolid, Spain \\ *Corresponding author.
}

\section{ABSTRACT}

Background and Objectives: "Noise power" (NP) is a measure that allows the assessment of the fast-firing synchronization of neural oscillations. We aimed to replicate higher gamma NP values in frontal and midline regions in patients with schizophrenia and re-evaluate its specificity to this disorder. We also aimed to assess the relationship of higher gamma NP values with drug treatment, chronicity, cognition and symptoms.

Methods: Gamma NP values were obtained from electroencephalograms recorded during an oddball paradigm from 29 patients with schizophrenia, 27 with bipolar disorder and 36 healthy controls. We compared these values between the groups to evaluate the specificity to diagnosis. Altered gamma NP values were compared between the patients who had and had not received different treatments to assess the relationship with drug treatment.

\footnotetext{
${ }^{3}$ Received 4 September 2020;Accepted 16 November 2020 https://doi.org/10.1016/j.ejpsy.2020.11.003
} 
We also analyse the correlation between gamma NP values and chronicity, symptoms, and cognition.

Results: Compared to controls, patients with schizophrenia presented increased gamma NP values in frontal and parietal midline regions, while bipolar patients showed increased gamma NP in the left frontal region. There was no significant relationship between drug treatment or chronicity with altered values. Increased gamma NP correlated with higher negative symptom scores in the schizophrenia group, but not with cognitive impairment in any of the groups of patients.

Conclusions: We replicated an increase in gamma NP in patients with schizophrenia and found that this alteration was also present in a milder form in bipolar patients. These alterations seem to be independent of pharmacological treatment and illness duration.

Keywords: Gamma band; Noise power; Schizophrenia; Bipolar disorder; Pharmacological treatment

Abbreviations: CPZ, chlorpromazine; DMN, default mode network; EEG, electroencephalogram; E/I, excitation and inhibition; EOG, electrooculogram; ICA, independent component analysis; NP, noise power; SNR, signal-to-noise ratio.

\section{Introduction}

Cognitive processes are based on accurate temporal relations of neural responses that are established by dynamic neural oscillations, ${ }^{1}$ especially in the gamma-frequency band $(30-100 \mathrm{~Hz}){ }^{2}$ This cortical gamma oscillatory activity depends on a balance of neuronal excitation and inhibition ( $E / I$ balance), a fundamental mechanism for information processing in the brain. ${ }^{3-}$ ${ }^{5}$ Although at the level of neurons the $\mathrm{E} / \mathrm{I}$ balance can be defined in a simplified way as the maintenance of appropriate ratios of excitatory versus inhibitory synaptic inputs, at the level of neural circuits is highly multidimensional and complex. ${ }^{6,7}$ Disturbances in this balance could be due to increased excitatory (glutamatergic) signaling, or to a reduction in inhibition signaling ( $\gamma$ aminobutyric acid -GABAergic) ${ }^{8}$ exercised by parvalbumin-positive interneurons that regulates pyramidal neurons firing, where NMDA receptors seems to play an important role. ${ }^{9}$ Consistent with this notion, a disruption in this $\mathrm{E} / \mathrm{I}$ balance leading to aberrant oscillatory activity in the gamma range has been proposed in schizophrenia. ${ }^{10-12}$ Correspondingly, alterations in the gamma band have also been proposed to play a role in the pathophysiology and cognitive dysfunction in schizophrenia and bipolar disorder. ${ }^{13-15}$ Thus, the assessment of gamma cortical oscillatory activity related to $\mathrm{E} / \mathrm{I}$ balance is 
considered a useful tool in the assessment of the electrophysiological basis of brain functioning and its alterations in schizophrenia and other mental disorders. ${ }^{16}$

Electroencephalography (EEG) is a widely used technique to measure oscillatory neuronal activity, providing high temporal resolution. ${ }^{17}$ Within the EEG frequency spectrum, gamma band oscillations are considered of special importance in neuroscience for a variety of reasons: (1) they are effective in supporting synchronization of neuronal firing, ${ }^{18}$ (2) seem to be involved in organizing the local neural circuits or assemblies that underlie higher brain functions, ${ }^{19}$ and (3) can be evoked or induced by sensory stimulation and cognitive tasks. ${ }^{20}$ In addition, numerous studies have reported abnormalities in the gamma band in schizophrenia, related to clinical symptoms and cognitive impairment. ${ }^{1,21-23}$

Research on the cognitive function of the human brain using EEG eventrelated potential (ERP) methods during task performance is generally focused on the analysis of stimulus-locked components. However, the activity registered after a target stimulus is composed not only of event-related activity, but also of activity linked to other concurrent cognitive processes inefficient for task resolution. ${ }^{24,25}$ Thus, other studies have been directed to investigate the neuronal activity generated during information processing that does not seem to be directly related in time to the stimulus, and has been generally considered simply as noise. ${ }^{26}$ This neuronal "noise", different from the non-neuronal or artifactual noise, represents variations in brain activity that occur apparently at random, with no clear relationship to the assessed phenomenon. ${ }^{27}$ In fact, the assessment of this noise magnitude, which is subsequently denoted as 'noise power' (NP), has provided relevant information in the investigation of neural cortical activity in schizophrenia. ${ }^{28-}$ 31

Previously, our group has reported elevated NP values in the gamma band over frontal and midline regions associated with cognitive deficit and clinical symptoms in minimally treated patients with schizophrenia. ${ }^{28,30,32}$ These results suggested that increased background neural oscillatory activity -unrelated to task performance- in the gamma band may be related to worst cognitive and clinical status of patients with schizophrenia.

In the present study we aimed to replicate these findings in a completely new sample of participants, comparing gamma NP values among schizophrenia patients, bipolar patients and healthy controls in order to reevaluate the specificity of altered gamma NP values to clinical diagnosis and their correlation with symptoms and cognition using a denser EEG array (29 electrodes) than in our previous reports. On the other hand, since neural oscillations may be affected by a number of factors, we also aimed to assess 
the possible relationship between the altered gamma NP values with psychotropic drug therapy and illness duration.

Based on evidence of an $\mathrm{E} / \mathrm{I}$ imbalance in schizophrenia ${ }^{33,34}$ related to an altered neural synchronization, ${ }^{35,36}$ and on findings from our previous research our main hypothesis was that gamma NP values during an oddball paradigm would be higher in schizophrenia but not in bipolar patients in comparison to healthy controls. Additionally, we expected to find that if these NP alterations reflect neurophysiological changes due to the disease, they were not explained by drug treatment nor illness duration. Our results could give credence to gamma NP alterations as a specific pathophysiological marker for schizophrenia.

\section{Material and methods}

\section{Subjects}

The sample of participants in this study included 29 chronic schizophrenia patients (19 males), 27 euthymic type 1 bipolar patients (17 males), and 36 healthy subjects ( 23 males). All participants reported no hearing problems. Healthy controls were recruited through newspaper advertisements. Patients were diagnosed by one of the psychiatrists from the research group (VM), who was the treating clinician in most of the cases, through clinical interviews according to the criteria of the Diagnostic and Statistical Manual of Mental Disorders, 5th edition. ${ }^{37}$ The demographic and clinical characteristics of the sample are shown in Table 1.

In patients, drugs and doses were stable during the 3 months prior to the EEG recordings. At the time of inclusion, chronic schizophrenia patients were all receiving atypical antipsychotics, 8 received antidepressants and 17 benzodiazepines. All the bipolar patients were euthymic: 16 were treated with antipsychotics, while 11 were not receiving this treatment and had not received it for at least the last six months, 17 were being treated with lithium, 14 with anticonvulsants, 11 with antidepressants and 12 with benzodiazepines (Table 1).

As in other previous studies of our group, the exclusion criteria were: (i) any neurological illness; (ii) history of cranial trauma with loss of consciousness longer than one minute; (iii) past or present substance abuse, except nicotine or caffeine; (iv) total intelligence quotient (IQ) under 70; (iv) for patients, any other psychiatric process; and ( $v$ ) for controls, any current psychiatric or neurological diagnosis and/or treatment with drugs known to act on the central nervous system. 
Table 1 Sociodemographic, clinical and cognitive data of patients and healthy controls.

\begin{tabular}{|c|c|c|c|}
\hline & $\begin{array}{c}\text { Schizophrenia } \\
\text { patients } \\
(\mathrm{N}=29) \\
\end{array}$ & $\begin{array}{l}\text { Bipolar } \\
\text { patients } \\
(\mathrm{N}=27)\end{array}$ & $\begin{array}{l}\text { Healthy } \\
\text { controls } \\
(\mathrm{N}=36)\end{array}$ \\
\hline \multicolumn{4}{|l|}{ Demographic and clinical: } \\
\hline Male: Female ratio & $19: 10$ & $17: 10$ & 23:13 \\
\hline Age (years) & $41.00(8.198)$ & $45.52(9.916)^{\#}$ & 39.89 (8.528) \\
\hline Illness duration (months) & $173.57(105.529)$ & $193.91(105.23)$ & N/A \\
\hline Education (years) & $13.286(4.088)^{\#}$ & $13.823(3.395)^{\#}$ & $17.600(3.406)$ \\
\hline Paternal education (years) & $9.611(3.913)$ & $10.600(4.137)$ & $13.300(4.739)$ \\
\hline \multicolumn{4}{|l|}{ Pharmacology (N): } \\
\hline Antipsychotics & 29 & 16 & N/A \\
\hline Lithium & 0 & 17 & $N / A$ \\
\hline Benzodiazepines & 17 & 12 & N/A \\
\hline Anticonvulsants & 0 & 14 & N/A \\
\hline Antidepressants & 8 & 11 & N/A \\
\hline $\mathrm{CPZ}$ equivalents $(\mathrm{mg} / \mathrm{d})$ & $\begin{array}{c}416.464 \\
(203.522)^{\star \star}\end{array}$ & $\begin{array}{c}230.781 \\
(123.773)\end{array}$ & N/A \\
\hline \multicolumn{4}{|l|}{ Symptoms (PANSS): } \\
\hline Positive scale & $12.04(4.587)^{\star \star}$ & $7.33(0.840)$ & N/A \\
\hline Negative scale & $18.20(8.246)^{\star \star}$ & $8.94(2.508)$ & $\mathrm{N} / \mathrm{A}$ \\
\hline Total scale & $57.16(19.482)^{\star \star}$ & $30.33(3.236)$ & N/A \\
\hline \multicolumn{4}{|l|}{ Cognition (BACS): } \\
\hline Verbal memory & $33.61(11.874)^{\#}$ & $35.63(9.185)^{\#}$ & $49.21(8.018)$ \\
\hline Working memory & $15.36(4.112)^{\#}$ & $17.47(3.850)^{\#}$ & $22.26(3.048)$ \\
\hline Motor speed & $59.78(19.551)^{\#}$ & $66.53(13.397)^{\#}$ & $80.59(11.904)$ \\
\hline Verbal fluency & $17.04(5.340)^{\#}$ & $20.79(5.907)^{\#}$ & $28.70(6.292)$ \\
\hline Processing speed & $37.61(12.819)^{\#}$ & $41.53(12.317)^{\#}$ & $64.50(10.805)$ \\
\hline Problem solving & $16.46(4.734)$ & $16.26(2.978)$ & $17.82(3.270)$ \\
\hline Cognitive factor 1 & $-0.823(0.785)^{\#}$ & $-0.432(0.632)^{\#}$ & $0.870(0.506)$ \\
\hline Cognitive factor 2 & $-0.030(1.152)$ & $-0.160(0.823)$ & $0.112(0.980)$ \\
\hline Total IQ & $94.41(11.937)^{\#}$ & $98.05(9.925)^{\#}$ & $115.65(8.800)$ \\
\hline \multicolumn{4}{|l|}{ Neurophysiology: } \\
\hline Pz P3b amplitude $(\mu \mathrm{V})$ & $1.222(1.849)$ & $1.563(1.714)$ & $1.780(2.024)$ \\
\hline Target trials included & $75.5(8.12)^{\#}$ & $84.04(19.57)$ & $93.69(12.20)$ \\
\hline Percentage of correct responses & $83.46(18.83)^{\#}$ & $90.82(11.11)^{\#}$ & $99.23(0.99)$ \\
\hline Percentage of omissions & $16.54(18.83)^{\#}$ & $9.18(11.11)^{\#}$ & $0.7711(0.99)$ \\
\hline Percentage of false alarm errors & $15.57(18.57)^{\#}$ & $12.87(16.80)^{\#}$ & $1.43(1.97)$ \\
\hline Average reaction time (ms) & $298.49(46.87)^{\#}$ & $276.68(58.75)^{\#}$ & $241.13(41.84)$ \\
\hline
\end{tabular}

Results are displayed as the mean (SD); N/A = not applicable; PANSS = Positive and Negative Syndrome Scale; BACS = Brief Assessment in Cognition in Schizophrenia Scale; WAIS-III Wechsler Adult Intelligence Scale.

${ }^{*} p<0.05$ and ${ }^{* *} p<0.01$ between schizophrenia and bipolar groups.

$\# p<0.05$ in comparison with healthy controls.

We obtained written informed consent from all participants after providing full printed and verbal information. The local ethical committee approved the study according to The Code of Ethics of the World Medical Association (Declaration of Helsinki).

\section{Clinical, pharmacological and cognitive assessment}

We scored the clinical status of patients using the Positive and Negative Syndrome Scale (PANSS). ${ }^{38}$ The type of drug treatment (antipsychotics, 
lithium, benzodiazepines, anticonvulsants and/or antidepressants) was recorded after consultation with the patients' psychiatrist or recent medical history. The dose of antipsychotic treatment was transformed into equivalents of chlorpromazine mg per day.

Cognition was assessed in all participants using the Spanish version of the Brief Assessment in Cognition in Schizophrenia Scale (BACS), ${ }^{39}$ including the following cognitive dimensions and tasks: verbal memory (word list learning), working memory (digit span), motor speed (token motor task), verbal fluency (word categories), attention and processing speed (symbol coding) and executive function/problem solving (Tower of London). IQ was estimated using the Spanish version of the Wechsler Adult Intelligence Scale (WAIS-III). ${ }^{40}$

\section{EEG acquisition and processing}

\section{EEG recording}

EEG recordings were acquired using a Brain Vision ${ }^{\circledR}$ equipment (Brain Products $\mathrm{GmbH}$; Munich, Germany) mounted in a 29-electrode cap (Electro-Cap International, Inc.; Eaton, Ohio, USA) and placed according to the modified 10/20 International System at Fp1, Fp2, F7, F3, Fz, F4, F8, FC5, FC1, FCZ, FC2, $\mathrm{FC6}, \mathrm{T7}, \mathrm{C3}, \mathrm{Cz}, \mathrm{C4}$, T8, CP5, CP1, CP2, CP6, P7, P3, PZ, P4, P8, O1, OZ, and O2. Two additional electrooculography (EOG) electrodes were placed for monitoring blinks and both vertical and lateral eye movements.

EEG signals were referenced online to $\mathrm{Cz}$ electrode and recorded continuously at a sampling rate of $500 \mathrm{~Hz}$. A high-pass hardware filter was applied (cut-off frequency $=0.50 \mathrm{~Hz}$; time constant $=0.3 \mathrm{~s}$; slope $=12 \mathrm{~dB} / \mathrm{oct}$ ). Electrode impedance was kept under $5 \mathrm{k} \Omega$ during the recordings.

A 13 min long 3-stimuli auditory oddball paradigm was employed to elicit the P3b component of the P300 evoked potential. Participants were exposed to random series of 600 binaural tone bursts (duration $50 \mathrm{~ms}$, rise and fall time $5 \mathrm{~ms}$, intensity $90 \mathrm{~dB}$ and onset asynchrony of 1000 and $1500 \mathrm{~ms}$ ) comprising target $(500 \mathrm{~Hz})$, distracter $(1000 \mathrm{~Hz})$ and standard $(2000 \mathrm{~Hz})$ tones with probabilities of $0.20,0.20$ and 0.60 , respectively. ${ }^{41}$ Participants were comfortably seated in a quiet room and were instructed to keep their eyes closed and click a mouse button whenever they detected the target tone.

\section{Signal pre-processing and processing}

EEG signals were off-line re-referenced to the average activity of all sensors and filtered using a $0.5-70 \mathrm{~Hz}$ band-pass filter. Subsequently the recordings were divided into $650 \mathrm{~ms}$ epochs starting $50 \mathrm{~ms}$ before the target stimulus onset. Only correctly identified target epochs were included in the analyses. Epochs were baseline corrected (50-ms pre-stimulus) and automatically rejected when exceeding a range of $\pm 70 \mu \mathrm{V}$ in any of the 29 channels. 
Next, to improve signal artefact correction, we applied an independent component analysis (ICA) including EEG and EOG data (Brain Vision ${ }^{\circledR}$ software). We subtracted all components clearly corresponding to eye movement. Finally, a visual inspection was performed to manually reject remaining epochs still presenting a clear artefact. Subject data were included in the analysis only if 40 or more useful epochs were still available for the target condition. The average number of valid target tone segments per participant was 87 (SD 17.65) (Table 1).

\section{P3b component calculation}

The P3b component was calculated from the average of those valid segments for the target condition and defined as the mean amplitude in the 300-400 ms interval. The individual averaged data were then grand-averaged to calculate the P3b component of each study group (Fig. S1).

\section{Gamma noise power (NP) calculation}

As described in previous articles ${ }^{25,32}$ the epoch-segmented data were band filtered ( $35-45 \mathrm{~Hz}$ for the gamma band) and a spectrum analysis was applied using a fast Fourier transform (FFT) to estimate the total power in the gamma band expressed in $\mu \mathrm{V}^{2}$.

We calculated NP following the previous recommendations, ${ }^{31,42}$ based on signal-to-noise ratio (SNR) and the average total power in the 300-400 ms post-target stimulus segment. Both measurements were estimated using the Brain Vision ${ }^{\circledR}$ software (Brain Products $\mathrm{GmbH}$; Munich, Germany. ${ }^{25,32}$

Since in the EEG recording neither the signal nor the noise powers are exactly known, average NP must be estimated with statistical methods. The signal power corresponds to the mean-squared amplitude of a series of the averaged trials, ${ }^{26}$ so that it was estimated by calculating the mean power of the averaged evoked single trials. ${ }^{24} \mathrm{NP}$ could then be computed by subtracting this signal power from the mean total power of the single sweep:

$$
\text { Avg NP=Avg Total Power - Avg Signal Power }
$$

SNR represents the quotient of the average signal power divided by the average noise power:

$$
S N R=\frac{\text { Avg Signal Power }}{\operatorname{Avg~NP}}
$$

and from Eq. (1): 
Anexo 3

$$
\text { Avg Signal Power }=\text { Avg Total Power }- \text { Avg NP }
$$

then, NP can be estimated by

$$
\text { Avg NP }=\frac{\text { Avg Total Power }}{\text { SNR+1 }}
$$

Thereby, using formula (4) in every individual participant, we calculated the averaged NP for each electrode in the gamma band from the already extracted average total power and SNR values.

\section{Statistical analysis}

Age and sex distribution were compared between groups using an analysis of variance (ANOVA, Bonferroni post-hoc) and a chi-square $\left(\mathrm{X}^{2}\right)$ test, respectively. Years of education, BACS scores, P300 amplitude, and performance variables during the oddball task were contrasted between groups also using an ANOVA (Bonferroni post-hoc). PANSS scores, treatment doses and illness duration were compared between the schizophrenia and bipolar groups using t-tests for independent samples. These statistical analyses were performed using IBM SPSS Amos 24 for Windows.

Kolmogorov-Smirnov tests for gamma NP values resulted in a nonnormal distribution for all 29-electrode measurements. Therefore, comparisons between groups on gamma NP were made using a nonparametric test (permutation test based on $t$ statistics) using the FieldTrip toolbox for MATLAB. ${ }^{43}$ Here, a Monte-Carlo estimate of the significance probabilities and/or critical values were calculated based on randomizing our data 1000 times between the study conditions. Type I error across multiple comparisons was controlled using Bonferroni correction ( $p=0.05 / 29=0.002$ ).

To reduce the number of cognitive factors (the six BACS dimensions), we performed a principal component analysis (PCA) and saved each of the participants' factorial scores for further analysis.

Spearman's rho correlation coefficient was then used to assess the relationship between clinical (treatment dose, illness durations and PANSS scores) and cognitive (factor scores) variables, and gamma NP values in those channels with a significant $p$-value in the previous step. Additionally, we studied the possible relationship between the altered NP values with the following performance variables during the oddball task: average reaction time to target stimulus, and percentages of correct responses, omissions (false negatives) and false alarm errors (false positives).

To assess a possible relationship between the use of psychotropic medication with the gamma NP values, we performed a group comparison between patients receiving or not each type of drug (antipsychotics, lithium, benzodiazepines, anticonvulsants and antidepressants). In order to make this 
comparison with the larger number of subjects, we included all patients regardless of their diagnosis. We performed these comparisons using new non-parametric permutation analyses based on t-tests (FieldTrip toolbox).

Finally, multiple regression models were carried out to analyse the predictive capacity of the different clinical and cognitive factors (independent variables) on the gamma NP values (dependent variable). The regression models were made in those groups of patients and electrodes where significant alterations of gamma NP were found in the previous comparisons. The independent variables were: illness duration, chlorpromazine equivalents received, PANSS scores (positive and negative symptoms, as well as total score), estimated IQ, response parameters in the oddball task (percentage of correct responses, omissions and false alarm errors, as well as average reaction times), and the two general cognitive factors.

A database with the main data supporting the results is available (Mendeley Data, V2, doi: 10.17632/538wvc7rp8.2).

\section{Results}

There were no significant differences between the groups in terms of sex distribution $\left(X^{2}=0.041, p=0.98\right.$; Table 1$)$. Bipolar patients were significantly older than healthy controls (mean difference $=5.63 p=0.04$ ). Illness duration was not significantly different between the groups of patients (Table 1 ).

Patients with schizophrenia had received significantly higher mean doses (in CPZ equivalents) of antipsychotics than bipolar patients $(t=3.307$, $p=0.002$; Table 1). The distribution of psychotropic drugs among patients with schizophrenia and bipolar disorder is also shown in Table 1.

Patients showed statistically significant lower scores compared to healthy controls in total IQ and in all BACS domains except Tower of London. The principal component analysis of the BACS scores yielded two independent factors (eigenvalue greater than 1) that explained $57.4 \%$ and $17.6 \%$ of the variance, respectively. The first cognitive factor included the verbal memory, working memory, motor speed, verbal fluency, attention and processing speed BACS scores, while the second cognitive factor corresponded to the executive function/problem solving domain (Tower of London). The first cognitive factor scores were significantly lower in both groups of patients compared to healthy controls $(p<0.001)$. As for the performance during the oddball task, patients had worse reaction times, gave fewer correct responses and made more errors by omission and false alarms (Table 1 ).

\section{Between-group differences in gamma NP}

The gamma NP values for each of the EEG electrodes are shown in Table 2 and topographically represented in Fig. 1. Our Bonferroni-corrected, non- 
parametric comparisons revealed significantly higher values of gamma NP in $\mathrm{Fz}(\mathrm{t}=3.460, \mathrm{p}=0.002)$, and $\mathrm{Pz}(\mathrm{t}=2.997, \mathrm{p}=0.002)$ channels in patients with schizophrenia compared to healthy controls. Bipolar patients, on the other hand, showed significantly higher values of gamma NP only in the F7 electrode compared to healthy controls $(t=2.702, p=0.002)$. No significant differences, corrected for multiple comparisons, were found between patients with schizophrenia and bipolar disorder (Fig. 1).

\section{a) Noise Power}

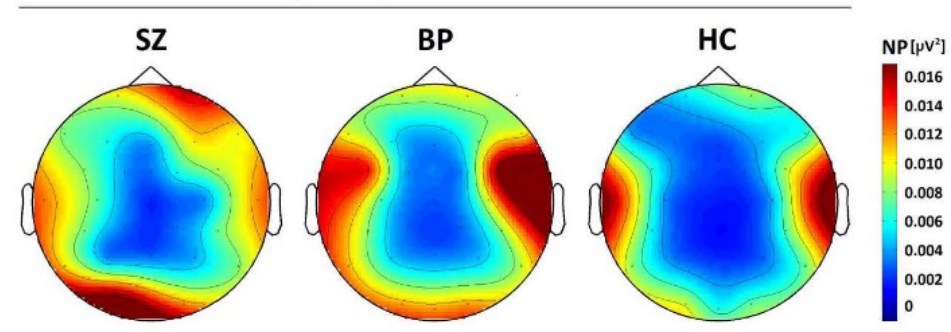

b) Differences

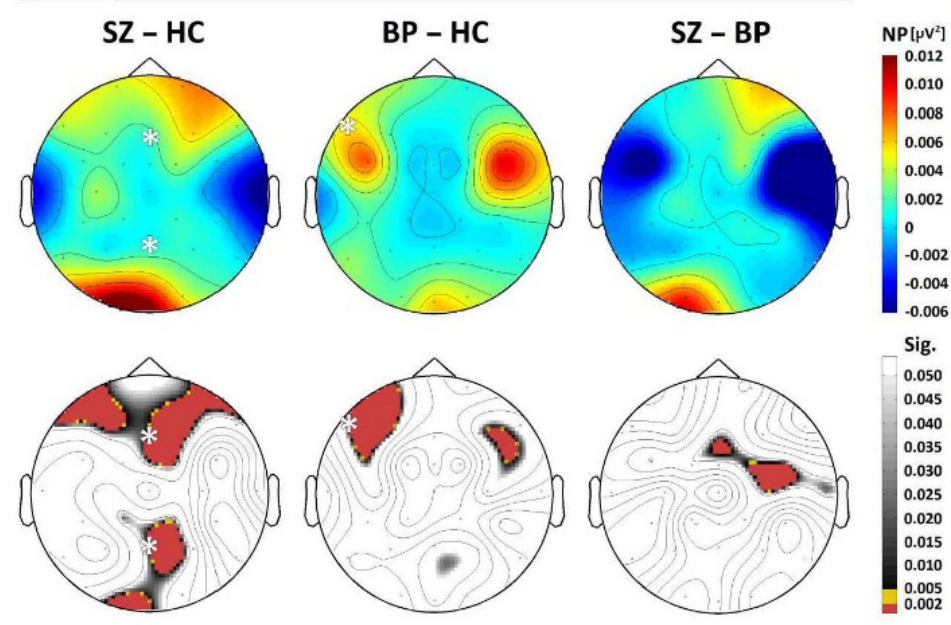

Figure 1 (a) Spatial scalp distribution of the grand-averaged gamma NP values for each group $\left(\mu \mathrm{V}^{2}\right)$. (b) Distribution of differences between the groups and their statistical significance ( $p$-values). The white asterisks represent the electrodes with significant differences after Bonferroni correction (SZ: schizophrenia patients, BP: bipolar patients and $\mathrm{HC}$ : healthy controls).

Relationship between gamma NP and pharmacological treatment and illness duration

Non-parametric contrast analyses between the groups treated and untreated with the different types of drugs can be seen in Fig. 2. No significant differences were found in the electrodes where we had previously found altered gamma NP values. 
We found no significant correlation between antipsychotic doses (expressed in CPZ equivalents) and gamma NP values in patients with schizophrenia in those electrodes where significant differences were found in the previous analysis. No significant correlations between illness duration and gamma NP values were found in patents with schizophrenia or bipolar disorder (Fig. S2).

Table 2 Gamma noise power values ( $y-N P)$ of patients and healthy controls $\left(\mu \mathrm{V}^{2}\right)$

\begin{tabular}{|c|c|c|c|c|c|c|}
\hline & \multicolumn{2}{|c|}{$\begin{array}{c}\text { Schizophrenia } \\
\text { patients } \\
\text { (N=29) }\end{array}$} & \multicolumn{2}{|c|}{$\begin{array}{l}\text { Bipolar } \\
\text { patients } \\
(\mathrm{N}=27)\end{array}$} & \multicolumn{2}{|c|}{$\begin{array}{l}\text { Healthy } \\
\text { controls } \\
(\mathrm{N}=36)\end{array}$} \\
\hline & Mean & SD & Mean & SD & Mean & SD \\
\hline$y$-NP FP1 & $0.00967^{\#}$ & $(0.01026)$ & $0.00868 \#$ & $(0.00665)$ & 0.00534 & $(0.00506)$ \\
\hline$y$-NP FP2 & $0.01509^{\#}$ & $(0.01557)$ & 0.00934 & $(0.00811)$ & 0.00814 & $(0.00922)$ \\
\hline y-NPF7 & $0.00780^{\#}$ & $(0.00967)$ & $0.00936 \#$ & $(0.01153)$ & 0.00394 & $(0.00304)$ \\
\hline$y-N P F 3$ & $0.00600 \#$ & $(0.00689)$ & $0.00508 \#$ & $(0.00345)$ & 0.00315 & $(0.00219)$ \\
\hline$Y$-NP FZ & 0.00384 & $(0.00200)$ & 0.00259 & $(0.00161)$ & 0.00230 & $(0.00160)$ \\
\hline$y$-NP F4 & $0.01044^{\#}$ & $(0.01720)$ & 0.00738 & $(0.00669)$ & 0.00462 & $(0.00433)$ \\
\hline$y$-NP F8 & 0.01003 & $(0.00953)$ & 0.00989 & $(0.00872)$ & 0.00634 & $(0.00821)$ \\
\hline$y$-NP FC5 & 0.00794 & $(0.01029)$ & $0.01438 \#$ & $(0.01528)$ & 0.00635 & $(0.00773)$ \\
\hline$y$-NP FC1 & 0.00388 & $(0.00290)$ & 0.00282 & $(0.00159)$ & 0.00276 & $(0.00367)$ \\
\hline y-NP FCZ & $0.00311 \#$ & $(0.00141)$ & 0.00243 & $(0.00179)$ & 0.00215 & $(0.00170)$ \\
\hline$y-N P F C 2$ & 0.00583 *\# & $(0.00699)$ & 0.00271 & $(0.00146)$ & 0.00263 & $(0.00252)$ \\
\hline$y$-NP FC6 & $0.00880^{*}$ & $(0.00945)$ & $0.01850 \#$ & $(0.02294)$ & 0.00860 & $(0.01041)$ \\
\hline Y-NP T7 & 0.01244 & $(0.01790)$ & 0.01527 & $(0.01167)$ & 0.01605 & $(0.01760)$ \\
\hline$y-N P C 3$ & 0.00715 & $(0.01447)$ & 0.00636 & $(0.00826)$ & 0.00374 & $(0.00487)$ \\
\hline$y-N P C Z$ & 0.00185 & $(0.00099)$ & 0.00179 & $(0.00088)$ & 0.00167 & $(0.00088)$ \\
\hline Y-NP C4 & $0.00356^{*}$ & $(0.00323)$ & 0.00952 & $(0.01600)$ & 0.00430 & $(0.00610)$ \\
\hline$y$-NP T8 & $0.01224^{*}$ & $(0.01862)$ & 0.02270 & $(0.01837)$ & 0.01843 & $(0.01808)$ \\
\hline$y$-NP CP5 & 0.00749 & $(0.01041)$ & 0.00750 & $(0.00778)$ & 0.00571 & $(0.00816)$ \\
\hline$y$-NP CP1 & 0.00320 \# & $(0.00325)$ & 0.00199 & $(0.00130)$ & 0.00185 & (0.00148) \\
\hline y-NP CP2 & 0.00324 & $(0.00419)$ & 0.00204 & $(0.00116)$ & 0.00179 & $(0.00108)$ \\
\hline$y$-NP CP6 & 0.00731 & $(0.01119)$ & 0.00765 & $(0.00672)$ & 0.00698 & $(0.01144)$ \\
\hline y-NP P7 & 0.01078 & $(0.01663)$ & 0.01138 & $(0.01366)$ & 0.00818 & $(0.00855)$ \\
\hline$y$-NPP3 & 0.00363 & $(0.00306)$ & 0.00421 & $(0.00434)$ & 0.00322 & $(0.00319)$ \\
\hline$y-N P P Z$ & $0.00276^{\#}$ & $(0.00178)$ & 0.00222 & $(0.00150)$ & 0.00175 & $(0.00086)$ \\
\hline$y$-NP P4 & 0.00415 & $(0.00432)$ & 0.00368 & $(0.00238)$ & 0.00290 & $(0.00210)$ \\
\hline$Y$-NP P8 & 0.00787 & $(0.00884)$ & 0.00861 & $(0.00675)$ & 0.00753 & $(0.00884)$ \\
\hline$y-N P O 1$ & $0.02057 \#$ & $(0.02518)$ & 0.01160 & $(0.01323)$ & 0.00909 & $(0.01025)$ \\
\hline$y-N P O Z$ & $0.01721^{\#}$ & $(0.02282)$ & 0.01180 & $(0.02045)$ & 0.00604 & $(0.00813)$ \\
\hline $\mathrm{y}$-NP O2 & 0.01213 & $(0.01244)$ & 0.01160 & $(0.01790)$ & 0.00729 & $(0.01599)$ \\
\hline
\end{tabular}

Results are displayed as mean (SD); comparisons between patients and healthy controls:

${ }^{*} p<0.05$ between schizophrenia and bipolar groups.

$\# p<0.05$ in comparison with healthy controls.

The comparisons that exceeded Bonferroni's correction are shown in bold and italics ( $p$ $<0.002$ ). 


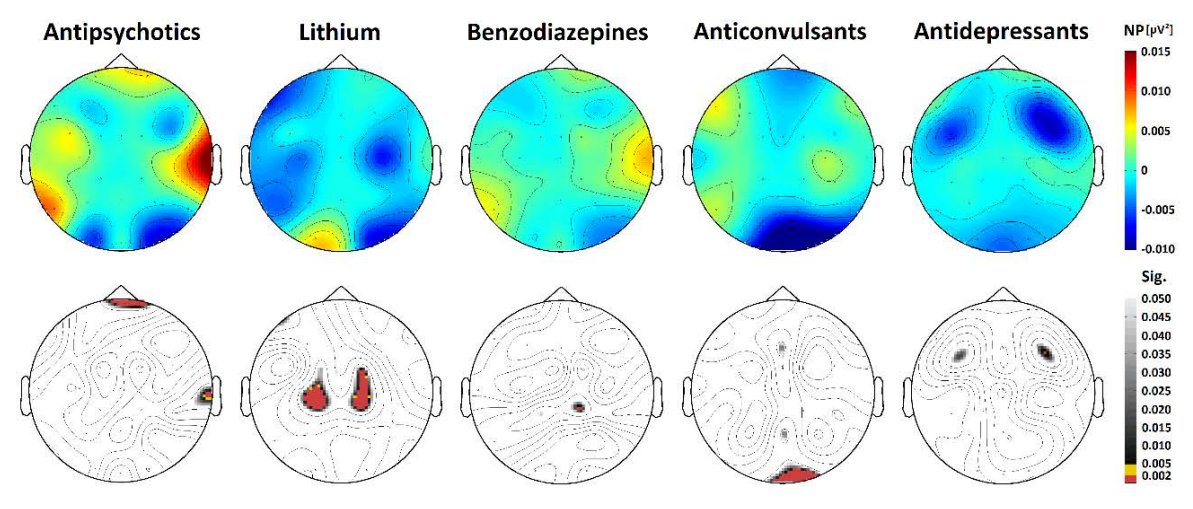

Figure 2 Topographical maps of the differences in grand-averaged gamma NP values in patients receiving minus not receiving each type of drug treatment (above) and their statistical differences (below). No electrodes showed statistical differences after Bonferroni correction. Gamma NP values $\left(\mu \mathrm{V}^{2}\right)$ above; $p$-values below.

\section{Relationship between gamma NP and clinical and cognitive factors}

Gamma NP values in patients with schizophrenia correlated significantly (after Bonferroni correction) with PANSS negative scores on F7 (rho $=0.642$, $\mathrm{p}=0.001)$, FC5 (rho $=0.612, \mathrm{p}=0.001), \mathrm{T} 7 \quad(\mathrm{rho}=0.649, \mathrm{p}<0.001), \mathrm{P7}$ (rho=0.627, $p=0.001$ ), P3 (rho=0.629, $p=0.001$ ), P4 (rho=0.581, $\mathrm{p}=0.002$ ) and $\mathrm{P} 8$ (rho $=0.712, \mathrm{p}<0.001$ ) electrodes, and with PANSS total scores on T7 (rho $=0.668, \mathrm{p}<0.001$ ), P7 (rho $=0.665, \mathrm{p}<0.001$ ) and P8 ( $r$ o $=0.618, p=0.001$ ) electrodes (Fig. 3). No correlation was found between gamma NP values and PANSS scores in bipolar patients. No significant correlations between gamma NP values and BACS cognitive factor scores were found for any of the patient groups. Similarly, no significant correlations were found between altered gamma NP values and any of the performance variables in the oddball task.

Finally, two multiple regression models were performed in the group of patients with schizophrenia on the altered values of gamma NP in the Fz and $\mathrm{Pz}$ electrodes. Neither model was able to explain significantly the gamma NP from any of the independent variables (clinical or cognitive) proposed. 

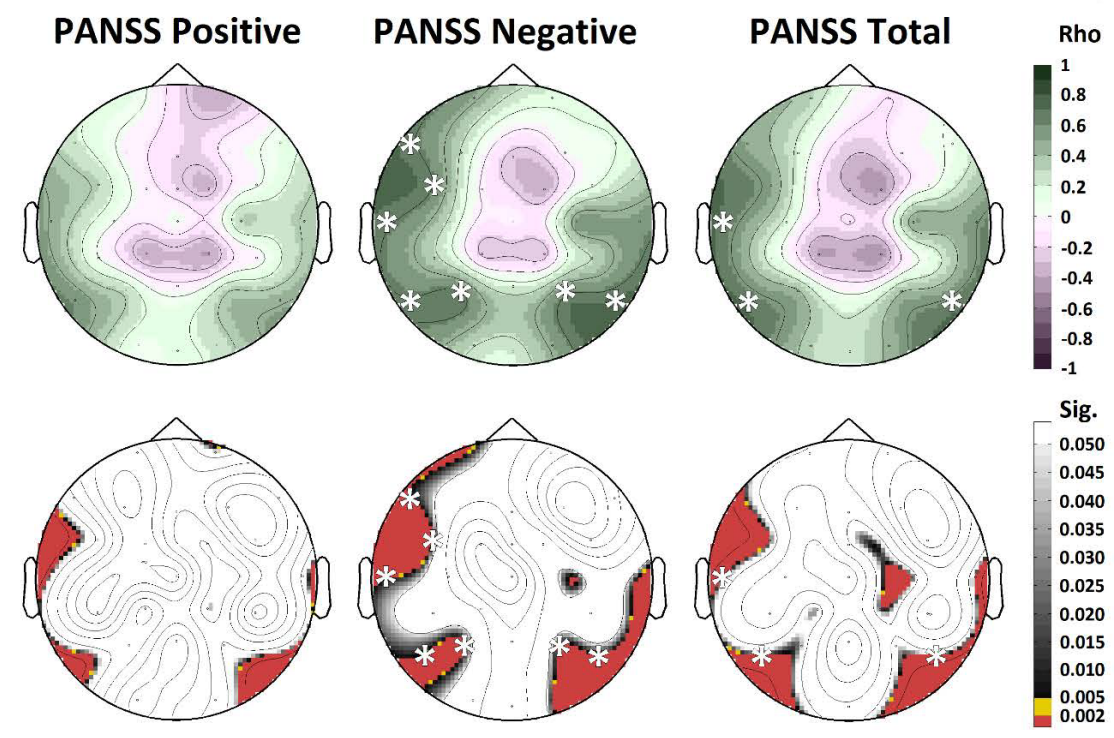

Figure 3 Correlation between gamma NP values and symptom scores in patients with schizophrenia. A significant correlation was found between gamma NP values in the left frontal and temporo-parietal regions and PANSS negative and total scores. The white asterisks represent the electrodes with significant correlation after Bonferroni correction. Spearman's rho above; $p$-values below.

\section{Discussion}

The study of measurements derived from cortical noise, such as noise power (NP) can be useful for the assessment of gamma power abnormalities ${ }^{32}$ which have previously been detected in schizophrenia and bipolar disorder. ${ }^{44,45} \mathrm{This}$ may, in turn, give insight into the disruption in the balance between excitatory and inhibitory mechanisms in psychosis. ${ }^{10}$ Thus, in our approach, NP has been defined as the amount of neural activity registered by the scalp EEG that is not time-locked during a cognitive task. In this study, we investigated the specificity of altered NP values in the gamma band for schizophrenia, compared to patients with bipolar disorder and healthy controls, and the possible relationship with pharmacological treatment and illness chronicity.

According to our results, patients with schizophrenia showed significantly higher gamma NP values in the frontal and parietal midline regions compared to healthy controls. This finding is in line with the evidence of altered neural synchronization in schizophrenia, ${ }^{23}$ and can be taken as suggestive of an imbalance of excitatory and inhibitory neuronal activity that is involved in the pathophysiology of this disorder. ${ }^{46} \mathrm{It}$ also was consistent with our previous reports of higher gamma NP values in the midline region in a completely different sample of patients, ${ }^{25,30}$ as well as with the higher gamma NP values in frontal regions in schizophrenia observed in other previous 
work. ${ }^{31}$ The regional distribution of these altered gamma NP values roughly corresponds to the default mode network (DMN). ${ }^{47,48}$ Failure to deactivate the DMN during the performance of a cognitive task has been described in schizophrenia, ${ }^{49}$ presumably related to a hampered GABAergic neurotransmission. ${ }^{50}$ Although the neurophysiological origins of gamma NP have not yet been clearly established, these findings suggest the existence of a common mechanism.

With respect to patients with bipolar disorder, our data showed a higher gamma NP in the left-frontal area when compared to healthy controls. This result may indicate the possible presence of an alteration of the $\mathrm{E} / \mathrm{I}$ balance in bipolar disorder similar to that found in patients with schizophrenia, although milder and with an apparent different regional distribution. Although we were unable to find a relationship with patients' PANSS scores and BACS factors, some previous studies based on the analysis of EEG activity in patients with bipolar disorder have also reported alterations in left frontal regions, in these cases related to clinical and cognitive features of this disorder. ${ }^{51-54}$ In prior reports, our group found no significant differences between these two groups using completely different samples. ${ }^{28}$ This discrepancy may be related to methodological differences since the previous analyses were based on a lower resolution EEG recording (17- channels versus the current 29 channels) and a factor analysis of NP values was used, ${ }^{28}$ while here we performed a different approach of Bonferroni-corrected comparisons of the NP values in each of the electrodes. These current results support a milder alteration of gamma NP in bipolar disorder.

Our present study was aimed at assessing the neuronal activity in the gamma band. Nonetheless, another research work on NP in different EEG bands reported higher NP values across the entire frequency spectrum in patients with schizophrenia when compared to controls. ${ }^{31}$ However, in a previous study from our group we were unable to detect significant differences between patients with schizophrenia and the control group when NP values were specifically compared in the theta band. ${ }^{30}$ To our knowledge, no other works directly compare NP values in any EEG frequency band in patients with schizophrenia or bipolar disorder. Even though we have not been able to find significant differences in NP between patients with schizophrenia and bipolar patients, a study investigating the resting-state EEG power within specified frequency bands reported an increase in total power in the beta and gamma bands in patients with bipolar disorder relative to patients with schizophrenia, ${ }^{45}$ which points to a possible interrelationship between alterations in different frequency bands that we have not been able to detect in previous studies.

The significantly higher values of gamma NP in patients were not related to antipsychotic treatment, when comparing those bipolar patients receiving 
and those not receiving this type of drug treatment (all patients with schizophrenia were taking antipsychotics). In addition, gamma NP alterations were not related to treatment with the other psychotropic drugs studied (BZP, lithium, antidepressants or anticonvulsants). No relationship was found either between gamma NP values and illness duration. These results suggest that the alterations found in gamma NP in patients are independent of the drug treatment and the duration of illness. Although, as discussed below, due to the small size of the groups receiving or not each drug, this conclusion should be taken with caution.

Patients showed significantly worse performance than healthy controls on most of the cognitive functions studied. However, we did not find a significant relationship between the altered gamma NP values and the overall cognitive factor, which included all BACS scores except the Tower of London. Previous reports from our group found altered gamma NP values in the frontal-lateral region correlated with poorer performance in working memory and problem solving in patients with schizophrenia. ${ }^{30,32}$ Methodological differences could account for the discrepancy between previous and current results. In the previous studies the specific domains of cognition were assessed, while in the present work only one cognition factor was used. Moreover, it should be noted that the interpretation of the results must be done with care since the gamma NP values analysed were obtained during an oddball paradigm (an attention and working memory task) but not during the performance of the BACS cognitive battery, including multiple cognitive domains under which more complex neural processes are probably executed. ${ }^{55}$ However, we neither found a correlation between the altered gamma NP values and the variables of the oddball task performance. In a previous report we found an association between gamma NP and a cognitive composite score (using the MATRICS battery). ${ }^{28}$ Thus, we cannot discard a relevant relationship between cognition and gamma NP not detected in the present sample.

Patients with schizophrenia showed a direct relationship between the elevated gamma NP values in the left-frontal and parietotemporal regions and negative symptoms. These findings correspond approximately to the direct correlation between higher gamma NP and negative PANSS scores previously reported in patients with first episode schizophrenia. ${ }^{32}$ Although we have not been able to obtain a relationship between altered gamma NP and poorer cognitive performance in patients, the relationship with negative symptoms would suggest that increased inefficient high-frequency neural activity probably hinders the neural processes underlying normal cognitive and affective expression.

Our study has some limitations. First, our NP measure is, by definition, calculated during a cognitive task, but was not assessed in a resting state for comparison, since there is evidence in the literature of altered spectral power 
in the resting state gamma band in patients with first episodes and chronic schizophrenia. ${ }^{10,56}$ Second, although higher gamma NP values may indicate an increased level of cortical activation to the detriment of satisfactory selection of neuron populations, it is still arguable whether NP thus described originates exclusively from cortical activity (background activity generated by a variation of peak-latency and/or actual response differences) or it may be affected by the contamination of external artifacts. ${ }^{26}$ This may have limited relevance in our results since our EEG data were pre-processed applying an ICA. Third, the results related to pharmacological treatment should be taken with care, since when comparing medicated and unmedicated patients we were not able to obtain groups taking only one type of medication, and for some comparisons the sample size was small. In addition, future studies should study the relationship between drugs and NP alterations for homogeneous samples of patients according to their diagnosis. Fourth, the present study assessed only chronic patients, and it has not been possible to make comparisons of gamma NP with previous stages of illness (clinical high-risk subjects or first episode patients in the case of schizophrenia). Lastly, we cannot discard a state- rather than trait-dependence of gamma NP alterations in patients with bipolar disorder, which could be analysed in longitudinal assessments of depressive, euthymic and manic stages. It is plausible that patients have an inefficient basal hyperactivity and therefore future work should study NP at a resting state, which would allow better estimation of the specificity of this measure for schizophrenia versus bipolar disorder. Likewise, the inclusion of first episodes and even a longitudinal study of cases would make it possible to better delineate the evolution of gamma NP alterations in patients.

\section{Conclusions}

We have confirmed previous findings showing that patients with schizophrenia have abnormally higher NP values in the gamma band in the midline, and that this alteration is present in a milder form in patients with bipolar disorder in frontal regions. These results are not explained by pharmacological treatment or illness duration. A plausible deficit in the mechanism of neural inhibition may underlay an inefficient cortical hyperactivity, and possibly, the increase of negative symptoms specifically in schizophrenia.

\section{Author contributions}

All authors contributed to the study conception. VM designed the study. Material preparation and data collection were performed by $B C, A L, M I, C C$ and AR. Data analysis was performed by $B C, A D$ and $V M$. The first draft of the manuscript was written by $B C, A D$ and VM. All authors commented on 
previous versions of the manuscript. All authors read and approved the final manuscript.

\section{Funding}

This work has been supported by the following grants: the "Instituto de Salud Carlos III" (PI18/00178), the "Gerencia Regional de Salud de Castilla y León" (GRS 1485/A/17 and GRS 1721/A/18), and the "Consejería de Educación de la Junta de Castilla y León" (VA057P17).

\section{Ethical considerations}

We declare that the present study was conducted in accordance with the Declaration of Helsinki and we certify that formal approval to conduct the experiments described have been obtained from the review board of the University Hospital of Valladolid and could be provided upon request.

\section{Conflicts of interest}

The authors declare that they have no conflict of interest.

\section{Acknowledgments}

None.

\section{Appendix A. Supplementary data}
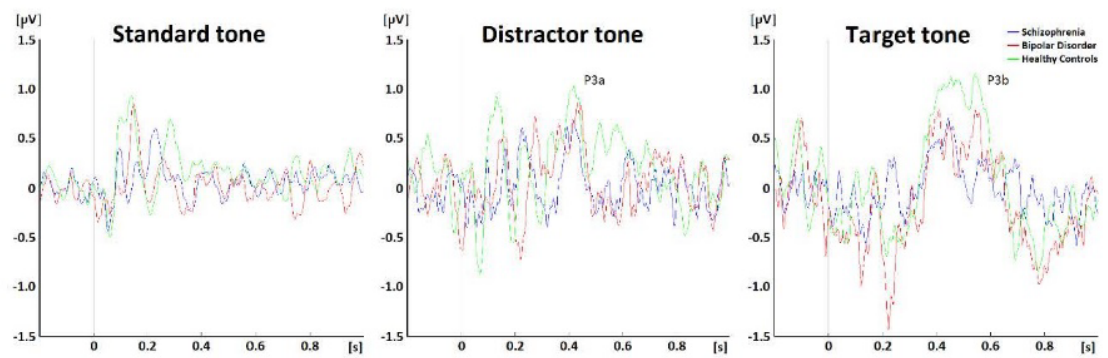

Figure \$1 Grand-average P300 waveform of patients and healthy controls for the standard, distractor and target tones. 
Schizophrenia
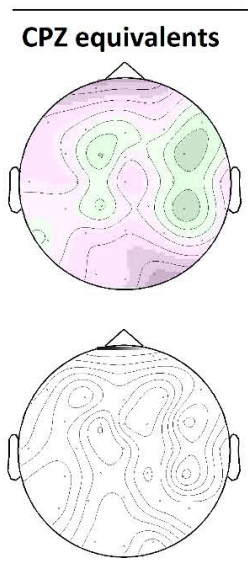
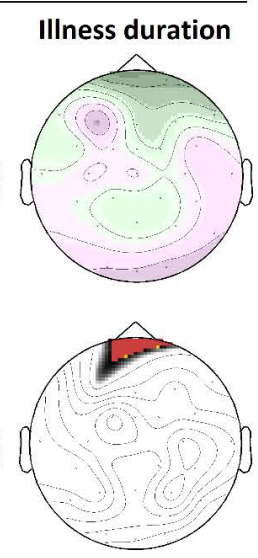

\section{Bipolar}
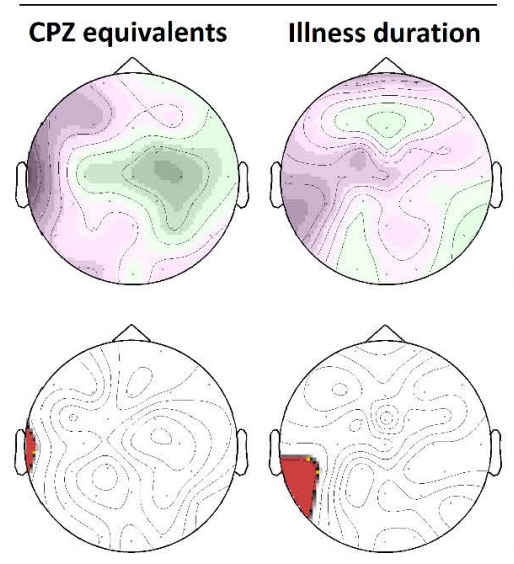

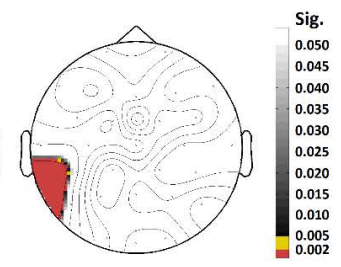

Figure S2 Correlations between gamma NP values and two clinical factors studied: the antipsychotic doses expressed in chlorpromazine (CPZ) equivalents and illness duration in patients with schizophrenia (left) and bipolar disorder (right). Spearman's rho above; p-values below.

\section{References}

1. Uhlhaas PJ, Singer W. Abnormal neural oscillations and synchrony in schizophrenia. Nat Rev Neurosci. 2010;11:100-13.

https://doi.org/10.1038/nrn2774.

2. Womelsdorf T, Schoffelen JM, Oostenveld R, Singer W, Desimone R, Engel AK, et al. Modulation of neuronal interactions through neuronal synchronization. Science 2007;316:1609-12. https://doi.org/10.1126/science.1139597.

3. Hensch TK, Fagiolini M, editors. Excitatory-Inhibitory Balance Synapses, Circuits, Systems. Springer US; 2004.

4. Tatti R, Haley MS, Swanson OK, Tselha T, Maffei A. Neurophysiology and regulation of the balance between excitation and inhibition in neocortical circuits. Biol Psychiatry 2017;81:821-31.

http://dx.doi.org/10.1016/j.biopsych.2016.09.017.

5. Ferguson BR, Gao WJ. PV interneurons: critical regulators of E/I balance for prefrontal cortex-dependent behavior and psychiatric disorders. Front Neural Circuits. 2018;12:1-13. https://doi.org/10.3389/fncir.2018.00037.

6. Sohal VS, Rubenstein JLR. Excitation-inhibition balance as a framework for investigating mechanisms in neuropsychiatric disorders. Mol Psychiatry 2019;24:1248-57. https://doi.org/10.1038/s41380-019-0426-0.

7. Gao R, Penzes P. Common mechanisms of excitatory and inhibitory imbalance in schizophrenia and autism spectrum disorders. Curr Mol Med. 2015;15:146-67. https://doi.org/10.2174/1566524015666150303003028.

8. Rubenstein JLR, Merzenich MM. Model of autism: increased ratio of excitation/inhibition in key neural systems. Genes, Brain Behav. 2003;2:255-67. https://doi.org/10.1034/j.1601-183X.2003.00037.x. 
9. Adell A. Brain NMDA receptors in schizophrenia and depression. Biomolecules 2020;10:1-27. https://doi.org/10.3390/biom10060947.

10. Grent-'t-jong T, Gross J, Goense J, Wibral M, Gajwani R, Gumley Al, et al. Resting-state gamma-band power alterations in schizophrenia reveal e/i-balance abnormalities across illness-stages. Elife 2018;7:e37799. https://doi.org/10.7554/eLife.37799.

11. Uhlhaas PJ, Singer W. Neuronal dynamics and neuropsychiatric disorders: toward a translational paradigm for dysfunctional large-scale networks. Neuron 2012;75:963-80. https://doi.org/10.1016/j.neuron.2012.09.004.

12. Gonzalez-Burgos G, Lewis DA. NMDA receptor hypofunction, parvalbuminpositive neurons, and cortical gamma oscillations in schizophrenia. Schizophr Bull. 2012;38:950-7. https://doi.org/10.1093/schbul/sbs010.

13. Uhlhaas PJ, Singer $W$. Neural synchrony in brain disorders: relevance for cognitive dysfunctions and pathophysiology. Neuron 2006;52:155-68. https://doi.org/10.1016/j.neuron.2006.09.020.

14. Selten M, Van Bokhoven $\mathrm{H}$, Kasri NN. Inhibitory control of the excitatory/inhibitory balance in psychiatric disorders. F1000Research 2018;7:116. https://doi.org/10.12688/f1000research.12155.1.

15. Murray JD, Wang XJ. Cortical circuit models in psychiatry: linking disrupted excitation-inhibition balance to cognitive deficits associated with schizophrenia. In: Anticevic A, Murray JD, editors. Computational Psychiatry: Mathematical Modeling of Mental Illness. Academic Press; 2018. p. 3-25. https://doi.org/10.1016/B978-0-12-809825-7.00001-8.

16. Allen P, Sommer IE, Jardri R, Eysenck MW, Hugdahl K. Extrinsic and default mode networks in psychiatric conditions: relationship to excitatory-inhibitory transmitter balance and early trauma. Neurosci Biobehav Rev. 2019;99:90-100. https://doi.org/10.1016/j.neubiorev.2019.02.004.

17. Winterer G, Mccarley RW. Electrophysiology of schizophrenia. In: Weinberger DR, Harrison P, editors. Schizophrenia. 3rd ed. Wiley-Blackwell; 2011, p. 311-33.

18. Fries $P$, Neuenschwander $S$, Engel AK, Goebel $R$, Singer W. Rapid feature selective neuronal synchronization through correlated latency shifting. Nat Neurosci. 2001;4:194-200. https://doi.org/10.1038/84032.

19. Lee K-H, Williams LM, Breakspear M, Gordon E. Synchronous gamma activity: a review and contribution to an integrative neuroscience model of schizophrenia. Brain Res Rev. 2003;41:57-78. https://doi.org/10.1016/S0165-0173(02)00220-5.

20. Başar E. A review of gamma oscillations in healthy subjects and in cognitive impairment. Int. J. Psychophysiol. 2013;90:99-117. https://doi.org/10.1016/J.IJPSYCHO.2013.07.005.

21. Spencer KM, Nestor PG, Niznikiewicz MA, Salisbury DF, Shenton ME, McCarley RW. Abnormal neural synchrony in schizophrenia. J Neurosci. 2003;23:7407-11. https://doi.org/10.1523/JNEUROSCI.23-19-07407.2003.

22. Basar-Eroglu C, Brand A, Hildebrandt H, Karolina Kedzior K, Mathes B, Schmiedt C. Working memory related gamma oscillations in schizophrenia patients. Int. J. Psychophysiol. 2007;64:39-45. https://doi.org/10.1016/J.IJPSYCHO.2006.07.007.

23. Gandal MJ, Edgar JC, Klook K, Siegel SJ. Gamma synchrony: towards a translational biomarker for the treatment-resistant symptoms of schizophrenia. 
Neuropharmacology 2012;62:1504-18. https://doi.org/10.1016/J.NEUROPHARM.2011.02.007.

24. Winterer G, Ziller M, Dorn H, Frick K, Mulert C, Dahhan N, et al. Cortical activation, signal-to-noise ratio and stochastic resonance during information processing in man. Clin Neurophysiol. 1999;110:1193-203. https://doi.org/10.1016/s1388-2457(99)00059-0.

25. Díez Á, Suazo V, Casado P, Martín-Loeches M, Molina V. Spatial distribution and cognitive correlates of gamma noise power in schizophrenia. Psychol Med. 2012;43:1175-85. https://doi.org/10.1017/S0033291712002103.

26. Winterer G, Ziller M, Dorn H, Frick K, Mulert C, Wuebben Y, et al. Schizophrenia: reduced signal-to-noise ratio and impaired phase-locking during information processing. Clin Neurophysiol. 2000;111:837-49. https://doi.org/10.1016/S1388-2457(99)00322-3.

27. Buzsáki $G$, Watson BO. Brain rhythms and neural syntax: implications for efficient coding of cognitive content and neuropsychiatric disease. Dialogues Clin Neurosci. 2012;14:345-67.

28. Suazo V, Lubeiro A, Jurado-Barba R, Moreno-Ortega M, Dompablo M, MoralesMuñoz I, et al. Elevated midline - parietal gamma band noise power in schizophrenia but not in bipolar patients. Eur Arch Psychiatry Clin Neurosci. 2016;266:743-53. https://doi.org/10.1007/s00406-016-0673-x.

29. Molina V, Bachiller A, Suazo V, Lubeiro A, Poza J, Hornero R. Noise power associated with decreased task-induced variability of brain electrical activity in schizophrenia. Eur Arch Psychiatry Clin Neurosci. 2016;266:55-61. https://doi.org/10.1007/s00406-014-0569-6.

30. Díez Á, Suazo V, Casado P, Martín-Loeches M, Perea MV, Molina V. Frontal gamma noise power and cognitive domains in schizophrenia. Psychiatry Res. 2014;221:104-13. https://doi.org/10.1016/J.PSCYCHRESNS.2013.11.001.

31. Winterer G, Coppola R, Goldberg TE, Egan MF, Jones DW, Sanchez CE, et al. Prefrontal broadband noise, working memory, and genetic risk for schizophrenia. Am. J. Psychiatry 2004;161:490-500. https://doi.org/10.1176/appi.ajp.161.3.490.

32. Suazo V, Díez Á, Martín C, Ballesteros A, Casado P, Martín-Loeches M, et al. Elevated noise power in gamma band related to negative symptoms and memory deficit in schizophrenia. Prog Neuropsychopharmacol Biol Psychiatry 2012;38:270-5. https://doi.org/10.1016/j.pnpbp.2012.04.010.

33. Foss-Feig JH, Adkinson BD, Ji JL, Yang G, Srihari VH, McPartland JC, et al. Searching for cross-diagnostic convergence: neural mechanisms governing excitation and inhibition balance in schizophrenia and autism spectrum disorders. Biol Psychiatry 2017;81:848-61. https://doi.org/10.1016/j.biopsych.2017.03.005.

34. Krystal JH, Anticevic A, Yang GJ, Dragoi G, Driesen NR, Wang XJ, et al. Impaired tuning of neural ensembles and the pathophysiology of schizophrenia: a translational and computational neuroscience perspective. Biol Psychiatry 2017;81:874-85. https://doi.org/10.1016/j.biopsych.2017.01.004.

35. Steullet P, Cabungcal JH, Coyle J, Didriksen M, Gill K, Grace AA, et al. Oxidative stress-driven parvalbumin interneuron impairment as a common mechanism in models of schizophrenia. Mol Psychiatry 2017;22:936-43.

https://doi.org/10.1038/mp.2017.47. 
36. Hirvonen J, Wibral M, Palva JM, Singer W, Uhlhaas P, Palva S. Whole-brain source-reconstructed MEG-data reveal reduced long-range synchronization in chronic schizophrenia. eNeuro 2017;4:1-14.

https://doi.org/10.1523/ENEURO.0338-17.2017.

37. American Psychiatric Association. Diagnostic and Statistical Manual of Mental Disorders. 5th ed. Washington, DC; 2013. https://doi.org/10.1176/appi.books.9780890425596.

38. Kay SR, Fiszbein A, Opler LA. The positive and negative syndrome scale (PANSS) for schizophrenia. Schizophr Bull. 1987;13:261-76. https://doi.org/10.1093/schbul/13.2.261.

39. Segarra N, Bernardo M, Gutierrez F, Justicia A, Fernadez-Egea E, Allas M, et al. Spanish validation of the Brief Assessment in Cognition in Schizophrenia (BACS) in patients with schizophrenia and healthy controls. Eur. Psychiatry 2011;26:6973. https://doi.org/10.1016/j.eurpsy.2009.11.001.

40. Wechsler D. Wechsler Adult Intelligence Scale-Third Edition. The Psychological Corporation. San Antonio, TX; 1997.

41. Bachiller A, Poza J, Gómez C, Molina V, Suazo V, Hornero R. A comparative study of event-related coupling patterns during an auditory oddball task in schizophrenia. J. Neural Eng. 2015;12:016007. https://doi.org/10.1088/17412560/12/1/016007.

42. Möcks J, Gasser T, Köhler W. Basic statistical parameters of event-related potentials. J Psychophysiol. 1988;2:61-70.

43. FieldTrip toolbox, http://www.fieldtriptoolbox.org/ [accessed 7 January 2020].

44. McNally JM, McCarley RW. Gamma band oscillations: a key to understanding schizophrenia symptoms and neural circuit abnormalities. Curr Opin Psychiatry. 2016;29:202-10. https://doi.org/10.1097/YCO.0000000000000244.

45. Kam JWYY, Bolbecker AR, O'Donnell BF, Hetrick WP, Brenner CA, Donnell BFO, et al. Resting state EEG power and coherence abnormalities in bipolar disorder and schizophrenia. J Psychiatr Res. 2013;47:1893-901. https://doi.org/10.1016/j.psychires.2013.09.009.

46. Uhlhaas PJ, Singer W. Oscillations and neuronal dynamics in schizophrenia: the search for basic symptoms and translational opportunities. Biol Psychiatry 2015;77:1001-9. https://doi.org/10.1016/j.biopsych.2014.11.019.

47. Buckner RL, DiNicola LM. The brain's default network: updated anatomy, physiology and evolving insights. Nat Rev Neurosci. 2019;20:593-608. https://doi.org/10.1038/s41583-019-0212-7.

48. Raichle ME, MacLeod AM, Snyder AZ, Powers WJ, Gusnard DA, Shulman GL. A default mode of brain function. Proc Natl Acad Sci U S A. 2001;98:676-82. https://doi.org/10.1073/pnas.98.2.676.

49. Pomarol-Clotet E, Salvador R, Sarró S, Gomar J, Vila F, Martínez Á, et al. Failure to deactivate in the prefrontal cortex in schizophrenia: dysfunction of the default mode network? Psychol Med. 2008;38:1185-93. https://doi.org/10.1017/S0033291708003565.

50. Gonzalez-Burgos G, Fish KN, Lewis DA. GABA neuron alterations, cortical circuit dysfunction and cognitive deficits in s chizophrenia. Neural Plast. 2011; 2011:124. https://doi.org/10.1155/2011/723184.

51. Harmon-Jones E, Abramson LY, Nusslock R, Sigelman JD, Urosevic S, Turonie LD, et al. Effect of bipolar disorder on left frontal cortical responses to goals 
differing in valence and task difficulty. Biol Psychiatry 2008;63:693-8. https://doi.org/10.1016/j.biopsych.2007.08.004.

52. Nusslock R, Alloy LB, Goldstein K, Harmon-Jones E, Urosevic S, Abramson LY. Elevated left mid-frontal cortical activity prospectively predicts conversion to bipolar I disorder. J Abnorm Psychol. 2012;121:592-601. https://doi.org/10.1037/a0028973.

53. Koller-Schlaud K, Ströhle A, Bärwolf E, Behr J, Rentzsch J. EEG frontal asymmetry and theta power in unipolar and bipolar depression. J Affect Disord. 2020;276:501-10. https://doi.org/10.1016/j.jad.2020.07.011.

54. Canali P, Sarasso S, Rosanova M, Casarotto S, Sferrazza-Papa G, Gosseries O, et al. Shared reduction of oscillatory natural frequencies in bipolar disorder, major depressive disorder and schizophrenia. J Affect Disord. 2015;184:111-5. https://doi.org/10.1016/j.jad.2015.05.043.

55. Petersen SE, Sporns O. Brain networks and cognitive architectures. Neuron 2015;88:207-19. https://doi.org/10.1016/j.neuron.2015.09.027.

56. Baradits M, Kakuszi B, Bálint S, Fullajtár M, Mód L, Bitter I, et al. Alterations in resting-state gamma activity in patients with schizophrenia: a high-density EEG study. Eur Arch Psychiatry Clin Neurosci. 2019;269:429-37.

https://doi.org/10.1007/s00406-018-0889-z. 